\title{
The analysis of Toeplitz operators, commutative Toeplitz algebras and applications to heat kernel constructions
}

\author{
Dissertation \\ zur Erlangung des mathematisch-naturwissenschaftlichen \\ Doktorgrades \\ "Doctor rerum naturalium" \\ der Georg-August-Universität Göttingen \\ vorgelegt von
}

\section{Hassan A. Issa}

aus Bourj El Barajne, Lebanon

Göttingen 2012 
Date of the oral examination : 
Dedicated to

M. Ibn L. Hassan Al-Moaammal 



\section{Contents}

$\begin{array}{ll}\text { Introduction } & 3\end{array}$

1 Preliminaries $\quad 19$

1.1 Reproducing kernel Hilbert spaces . . . . . . . . . . . . . . . . . . . . 19

1.2 Bergman spaces . . . . . . . . . . . . . . . . . . 24

1.3 Segal-Bargmann space . . . . . . . . . . . . . . . . . 28

1.4 Bergman spaces over the unit ball of $\mathbb{C}^{n} \ldots \ldots \ldots \ldots$

2 Commuting Toeplitz operators with quasi-homogeneous symbols on the Segal-Bargmann space 39

2.1 Introduction . . . . . . . . . . . . . . . . 40

2.2 Preliminaries . . . . . . . . . . . . . . . . . 43

2.3 Necessary and sufficient conditions for the commuting problem . . . . . . . . 48

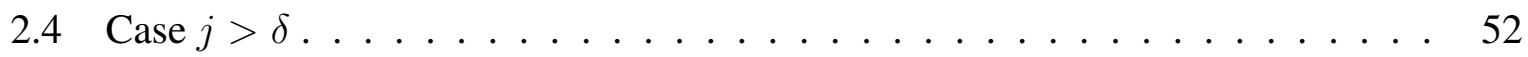

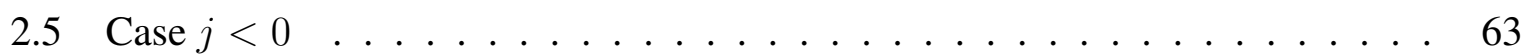

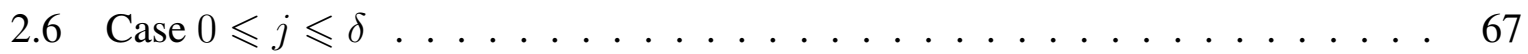

2.7 Examples and applications . . . . . . . . . . . . . . 69

3 Commutative algebras of Toeplitz operators $\quad 73$

3.1 Introduction . . . . . . . . . . . . . . . . . . 73

3.2 Commutative $C^{\star}$-algebras of Toeplitz operator . . . . . . . . . . . . . . 77

3.3 Commutative Banach algebras generated by Toeplitz operators with $k$-quasihomogeneous symbols . . . . . . . . . . . . . . 80

3.4 Commutative $C^{\star}$-algebras generated by true- $k$-Toeplitz operators . . . . . . . 85

4 „Heat kernel” for Toeplitz operators 93

4.1 Introduction . . . . . . . . . . . . . . . . . . 93

4.2 The Bargmann transform . . . . . . . . . . . . . . . . . . . . 96

4.3 Heat kernel by Toeplitz operator theory techniques . . . . . . . . . . . . 103

5 Compact Toeplitz operators for weighted Bergman spaces on bounded symmetric domains

5.1 Introduction . . . . . . . . . . . . . . . . . 125 
5.2 Preliminaries . . . . . . . . . . . . . . . . . 127

5.3 Upper estimation of the Berezin transform . . . . . . . . . . . . 131

5.4 Compactness criteria . . . . . . . . . . . . . 136

Appendix 


\section{Introduction}

${ }^{1}$ This dissertation is devoted to the study of certain analytic problems inspired by recent results in the theory of Toeplitz operators on the Bergman spaces. We attack the problem of commuting Toeplitz operators with quasi-homogeneous symbols on the Segal-Bargmann space over $\mathbb{C}$. For a fixed monomial $z^{l} \bar{z}^{k}$ we characterize the functions $\Psi$ of polynomial growth at infinity such that the Toeplitz operators $T_{\Psi}$ and $T_{z^{l} \bar{z}^{k}}$ commute on the space of all holomorphic polynomials over $\mathbb{C}$. Moreover, we construct commutative Banach and $C^{\star}$-algebras generated by Toeplitz operators acting on the Segal-Bargmann spaces over $\mathbb{C}^{n}$. We show that the class of symbols which generate the commutative Toeplitz $C^{\star}$-algebra generates also a commutative $C^{\star}$-algebra of operators acting on the true- $k$-Fock spaces. Furthermore, we use Toeplitz operator theory techniques to construct the heat kernel of a class of positive sub-elliptic differential operators. As an application, we obtain the heat kernel of the Grusin operator on $\mathbb{R}^{n+1}$ as well as that of the sub-Laplace operator on the $(2 n+1)$-dimensional Heisenberg group ( $n \in \mathbb{N}$ is arbitrary). Finally, we switch our attention to a compactness criteria for Toeplitz operators $T_{g}^{\nu}$ acting on the standard weighted Bergman spaces over bounded symmetric domains. We obtain an estimate for the Berezin tansform $\tilde{g}_{\nu_{0}}$ in terms of the operator norm of $T_{g}^{\nu}$ whenever $\nu$ and $\nu_{0}$ are suitable weights. As a consequence, we prove that for a bounded function $g$ on a bounded symmetric domain the compactness of $T_{g}^{\nu}$ is independent of the weight parameter $\nu$, for $\nu$ large enough.

For tackling these problems we use functional analytic methods as well as tools from operator and measure theory. Integral transforms such as Bargmann, Berezin, Fourier and Mellin transform are frequently used in our approach. Methods from differential equations and matrix analysis occur in our study of the ,heat kernel” for Toeplitz operators. Rudiments of the algebraic classification of bounded symmetric domains appear in the investigation of the uniform compactness for Toeplitz operators acting on such domains.

We start by introducing and motivating the above mentioned problems in more details. In order to facilitate our explanation of these problems we briefly indicate some basic facts in the theory of Toeplitz operators (c.f. Chapter 1).

Let $\Omega \subset \mathbb{C}^{n}$ be an open connected domain. For a suitable finite measure $\mu$ (c.f. Section 1.2) the Hilbert space of all holomorphic $\mu$-square integrable functions on $\Omega$ is called the Bergman space over $\Omega$ and is denoted by $H^{2}(\Omega, d \mu)$. Moreover, each pointwise evaluation map is continuous on $H^{2}(\Omega, d \mu)$ which turns the Bergman space into a reproducing kernel Hilbert

\footnotetext{
${ }^{1}$ The project has been supported by an "Emmy-Noether scholarship" of DFG (Deutsche Forschungsgemeinschaft).
} 
space (c.f. Section 1.1). For a measurable function $g$ on $\Omega$ and via the orthogonal projection $P$ from $L^{2}(\Omega, d \mu)$ onto $H^{2}(\Omega, d \mu)$, the Toeplitz operator $T_{g}$ is defined on a suitable domain in $H^{2}(\Omega, d \mu)$ by $T_{g} f:=P M_{g}$ where $M_{g}$ is the multiplication by $g$. The set of Toeplitz operators acting on the Bergman space over a certain domain is neither commutative nor closed under multiplication (composition of operators). In the analysis of Toeplitz operators two natural questions arise:

(I) Let $f$ and $g$ be two symbols belonging to a certain subclass of functions. What is the relation between $f$ and $g$ so that $T_{f}$ and $T_{g}$ commute?

(II) How to obtain a symbol class for which the algebra generated by Toeplitz operators with symbols in this class is commutative?

In recent years, there has been a considerable interest in the problem of characterizing the symbols of commuting Toeplitz operators over various domains $[8,9,16,17,60,61,66,126$, $129,130]$. In particular, the problem of commuting Toeplitz operators with bounded symbols on the Bergman space over the unit disc is not fully understood until now. Such a problem has been completely solved for the case of Toeplitz operators acting on the Hardy space $H^{2}\left(\mathbb{S}^{1}\right)$ (c.f. [186]). In [54], A. Brown and P. R. Halmos proved that two Toeplitz operators with bounded symbols commute on $H^{2}\left(\mathbb{S}^{1}\right)$ if and only if both symbols are analytic, or both are conjugate analytic, or one of them is a linear function of the other. In the case of the (un-weighted) Bergman space $A(\mathbb{D})$ over the unit disc $\mathbb{D}$ (where the measure $\mu$ is the normalized Lebesgue measure c.f. Section 1.4) the above mentioned result fails. In particular, if $f \in L^{\infty}(\mathbb{D})$ is radial then $T_{f}$ is diagonal w.r.t. the usual orthonormal basis of $A(\mathbb{D})$ so that Toeplitz operators with bounded radial symbols commute on $A(\mathbb{D})$. However, under an additional assumption on the symbols S. Axler and Z. Čučković showed that for a pair of commuting Toeplitz operators on $A(\mathbb{D})$ with bounded harmonic symbols, Brown and Halmos result is still valid [8].

A special case for the problem of commuting Toeplitz operators on $A(\mathbb{D})$ with bounded (non-harmonic) symbols has been considered by Z. Čučković and N. V. Rao in [66] and by I. Louhichi and L. Zakariasy in [130]. Roughly speaking, let $f$ be a monomial or more generally a bounded quasi-homogeneous function on $\mathbb{D}$. In $[66,130]$, sufficient and necessary conditions for a symbol $\psi \in L^{\infty}(\mathbb{D})$ have been obtained so that $T_{f}$ and $T_{\psi}$ commute on $A(\mathbb{D})$. The technique used is to express $\psi$ in an $L^{2}$-convergent series $\psi\left(r e^{i \theta}\right)=\sum_{j=-\infty}^{\infty} \psi_{j}(r) e^{i j \theta}$ of quasihomogeneous symbols ( $r$ and $\theta$ denote the polar coordinates in $\mathbb{C}$ ) and then investigate the commuting problem between $T_{f}$ and each $T_{\psi_{j}(r) e^{i j \theta}}$. The authors of [66] noticed that for each $j \in \mathbb{Z}$ there is at most one $\psi_{j}$ (up to a multiplication by a constant) such that the operators $T_{f}$ and $T_{\psi_{j}(r) e^{i j \theta}}$ commute on $A(\mathbb{D})$. They obtained the radial part $\psi_{j}$ in terms of the inverse Mellin transform of an expression formed of a product of Gamma functions. Moreover, using this approach they proved that for any $\Phi_{0}, \Phi \in L^{\infty}(\mathbb{D})$, where $\Phi_{0}$ is non-constant and radial, the operators $T_{\Phi_{0}}$ and $T_{\Phi}$ commute on $A(\mathbb{D})$ if and only if $\Phi$ is radial.

The above description motivates an analogous problem in the case of the Segal-Bargmann space over $\mathbb{C}$ (c.f. Section 1.3). First investigated by V. Bargmann [12, 13] and I.E. Segal 
[152], the Segal-Bargmann space over $\mathbb{C}^{n}$ (denoted by $H^{2}\left(\mathbb{C}^{n}, d \mu\right)$ ) is a Bergman space w.r.t. a Gaussian measure $\mu$ and is naturally isomorphic to the Fock space. The choice of $\mu$ allows the multiplication operator by the coordinate $z_{j}$ (the Toeplitz operator with symbol $z_{j}$ ) to be the adjoint of the differential operator $\partial_{z_{j}}$ (Toeplitz operator with symbol $\bar{z}_{j}$ ). Such operators are important in quantum mechanics because they satisfy the canonical commutational relations (CCR) $[12,13,62,160]$. Toeplitz operators on the Segal-Bargmann space have been studied by several authors [15-17, 34, 35, 94, 95] and the map $g \longrightarrow T_{g}^{t}$ has been considered in [29, 31$33,62,98]$.

The commuting problem of Toeplitz operators acting on $H^{2}\left(\mathbb{C}^{n}, d \mu\right)$ has been recently considered in the works of W. Bauer, T. Le and Y.J. Lee [16, 17]. It has been noticed that the growth of the symbol near infinity influences the results. For example, in [16] W. Bauer and T. Le proved that if $f$ and $g$ are two measurable functions having a linear exponential growth at infinity and $f$ is radial and non-constant then a sufficient and a necessary condition for $T_{f}$ and $T_{g}$ to commute is that $g$ is radial in each component. The case $n=1$ was treated in [17] where the implications , $g$ is radial" holds true. However, Example 5.6 in [17] shows that if we allow the operator symbol $g$ to have a higher growth at infinity then this result is no longer true even in the case where $f$ is bounded.

Inspired by these results and analogous to the case of the Bergman space over $\mathbb{D}$ we are interested in the following commuting problem of Toeplitz operators acting on the Segal-Bargmann space $H^{2}:=H^{2}(\mathbb{C}, d \mu)$ over the complex plane

(P0) Let $f_{m, \delta}\left(r e^{i \theta}\right)=r^{m} e^{i \delta \theta}=z^{l} \bar{z}^{k}$ be a monomial. Determine the operator symbols $\Psi$ having polynomial growth at infinity so that the Toeplitz operators $T_{f_{m, \delta}}$ and $T_{\Psi}$ commute on the space of all holomorphic polynomials $\mathbb{P}[z]$ in $H^{2}$.

Chapter 2 is devoted to the problem (P0). As a first step, we will reduce the above problem to the case $\delta \in \mathbb{N}_{0}$ and $\Psi$ is a quasi-homogeneous symbol. On the one hand, dealing i.g. with unbounded Toeplitz operators $T_{f_{m, \delta}}$ and $T_{\Psi}$ we prove the existence of a densely embedded Hilbert space $\mathcal{H}_{\epsilon} \hookrightarrow H^{2}$ containing $\mathbb{P}[z]$ on which the operator products $T_{\Psi} T_{f_{m, \delta}}$ and $T_{f_{m, \delta}} T_{\Psi}$ are well defined and continuous (c.f. Proposition 2.2.1 for a more general statement). As a consequence of this and the fact that the Berezin transform is injective on the set of bounded operators acting on $\mathcal{H}_{\epsilon}$, we will show that $T_{\Psi}$ and $T_{f_{m, \delta}}$ commute if and only if $T_{\bar{\Psi}}$ and $T_{f_{m,-\delta}}$ commute. This shows that it is sufficient to consider (P0) for the case $\delta \in \mathbb{N}_{0}$. On the other hand, we represent $\Psi$ as an $L^{2}$-convergent series $\Psi\left(r e^{i \theta}\right)=\sum_{j=-\infty}^{\infty} \Psi_{j}(r) e^{i j \theta}$ of quasi-homogeneous symbols. Upon applying the products $T_{\Psi} T_{f_{m, \delta}}$ and $T_{f_{m, \delta}} T_{\Psi}$ to the monomials it turns out that $T_{\Psi}$ and $T_{f_{m, \delta}}$ commute on $\mathbb{P}[z]$ if and only if for each $j \in \mathbb{Z}$ the operators $T_{f_{m, \delta}}$ and $T_{\Psi_{j}(r) e^{i j \theta}}$ commute on $\mathbb{P}[z]$ (c.f. Proposition 2.3.1 for a more general case). This result allows us to replace $(\mathrm{P} 0)$ by the following new problem

(P1) Let $f_{m, \delta}\left(r e^{i \theta}\right)=z^{l} \bar{z}^{k}=r^{m} e^{i \delta \theta}$ be a monomial. For each $j \in \mathbb{Z}$ determine the functions $\Psi_{j}$ defined a.e. on $\mathbb{R}_{+}$with at most polynomial growth at infinity such that the Toeplitz operators $T_{f_{m, \delta}}$ and $T_{\Psi_{j}(r) e^{i j \theta}}$ on $\mathbb{P}[z]$ commute. 
In our investigation of (P1), we fix $j \in \mathbb{Z}$ and we start by assuming that $T_{f_{m, \delta}} T_{\Psi_{j} e^{i j \theta}}\left(z^{k}\right)=$ $T_{\Psi_{j} e^{i j \theta}} T_{f_{m, \delta}}\left(z^{k}\right)$ for all $k \in \mathbb{N}_{0}$. From these relations we obtain a certain distribution of zeros of the Mellin transform of a radial function involving $\Psi_{j}(r) e^{-r^{2}}$. Using a result in [17], which replaces the Blaschke condition in the analysis on the unit disc, we derive from the previously obtained relations a functional equation for the Mellin transform of $\Psi_{j}(r) e^{-r^{2}}$ on some right half plane (Propositions 2.4.1, 2.5.1 and 2.6.1). In order to construct all possible solutions of the functional equation we have to distinguish between the cases $j>\delta, j<0$ and $0 \leqslant j \leqslant \delta$. By studying each case separately and under the assumption that $\Psi_{j}$ is at most of polynomial growth at infinity we obtain all possible solutions (Propositions 2.4.3 and 2.5.2 ). In all the cases, the radial function $\Psi_{j}$ is expressed as an inverse Mellin transform of an expression which is a product of Gamma functions and a trigonometric polynomial (Theorems 2.4.1, 2.5.2 and 2.6.1). From this collection of candidates obtained we search for those who produce reasonable symbols for Toeplitz operators so that the commutator $\left[T_{f_{m, \delta}}, T_{\Psi_{j} e^{i j \theta}}\right]$ vanishes on $\mathbb{P}[z]$. On the one hand, it turns out that for $j \in \mathbb{Z}$ sufficiently small there is no non-zero radial function $\Psi_{j}$ of polynomial growth at infinity such that $\left[T_{f_{m, \delta}}, T_{\Psi_{j} e^{i j \theta}}\right]=0$ (Theorem 2.5.1). On the other hand, if $j$ is large enough then there is at least one $\Psi_{j}$ of polynomial growth at infinity with this property (Corollaries 2.4.1 and 2.6.1). As it is indicated by Corollary 2.4.2 and Example 2.6.1 when $f_{m, \delta}$ is a monomial, there is an infinite number of indices $j \in \mathbb{Z}$ where the existence as well as the uniqueness (up to a constant factor) of a non-zero $\Psi_{j}$ is obtained. If we allow operator symbols of higher growth at infinity, and for a fixed $j$ we point out that in some of the cases there are more than one Toeplitz operator $T_{\Psi_{j}(r) e^{i j \theta}}$ commuting with $T_{f_{m, \delta}}$.

Chapter 2 contains many applications of the above mentioned results. Roughly speaking, we prove that for any $j \in \mathbb{Z}$ the radial component $\Psi_{j}$ can be extended to a complex analytic function on a neighborhood of $\mathbb{R}_{+}$(Corollary 2.7.1). In particular, they are uniquely determined by their restrictions to any open sub-interval of $\mathbb{R}_{+}$. In Example 2.4 .1 we show that in the case where $m \neq 0$ the condition $\left[T_{f_{m, 0}}, T_{\Psi}\right]=0$ is equivalent to $\Psi$ being radial (c.f. [17] for a more general result). Moreover, we recover the examples in [17] of radial functions $u$ such that $T_{u}$ commutes with another Toeplitz operator having a non-radial symbol (Example 2.7.1). Furthermore, we note that I. Louhichi and N. V. Rao conjectured that if two Toeplitz operators with bounded symbols acting on $A(\mathbb{D})$ commute with a third one, none of them being the identity, then they commute with each other [129]. Motivated by this conjecture, our results in this chapter allow us to construct a triple $\left(T_{f}, T_{g}, T_{h}\right)$ of Toeplitz operators with unbounded symbols such that $\left[T_{f}, T_{g}\right]=\left[T_{f}, T_{h}\right]=0$ but $\left[T_{g}, T_{h}\right] \neq 0$ (Examples 2.7.2 and 2.7.3).

One can also consider a family of Segal-Bargmann spaces $\mathcal{H}_{s}^{2}\left(\mathbb{C}^{n}\right):=H^{2}\left(\mathbb{C}^{n}, d \mu_{(s)}\right)$ parametrized w.r.t. the time parameter $s>0$ and the measure $\mu_{s}$ is a Gaussian measure (c.f. Section 1.3). These spaces are mapped to each other unitarly by a simple composition operator (c.f. (1.3.4)). For a measurable function $g$ on $\mathbb{C}^{n}$ we write $\mathcal{T}_{g}^{s}$ for the Toeplitz operator with symbol $g$ acting on $\mathcal{H}_{s}^{2}\left(\mathbb{C}^{n}\right)$. We remark that with the notation used above and in the case of the complex plane we have $H^{2}=\mathcal{H}_{1}^{2}(\mathbb{C})$ and $\mathcal{T}_{g}^{1}=T_{g}$.

In connection with problem (P1) one may ask whether the commuting problem is uniform 
w.r.t. the time parameter $s$. Roughly speaking, let $f_{m, \delta}$ be a monomial and suppose that for some $j \in \mathbb{Z}$ there is a function $\Psi_{j}(r) e^{i j \theta}$ of polynomial growth at infinity such that $T_{f_{m, \delta}}$ and $T_{\Psi_{j}(r) e^{i j \theta}}$ commute on $\mathbb{P}[z] \subset H^{2}$. Does it follow that $\mathcal{T}_{f_{m, \delta}}^{s}$ and $\mathcal{T}_{\Psi_{j}(r) e^{i j \theta}}^{s}$ commute on $\mathbb{P}[z] \subset \mathcal{H}_{s}^{2}(\mathbb{C})$ for some $s \neq 1$ ? The answer to this question is i.g. negative as it is indicated by the example given in Remark 2.4.2. However, when $\Psi_{j}(r) e^{i j \theta}$ is a polynomial, then by a composition formula for the product of Toeplitz operators with polynomial symbols (c.f. [63] and [14] for a more general result) the operators $\mathcal{T}_{f_{m, \delta}}^{s}$ and $\mathcal{T}_{\Psi_{j}(r) e^{i j \theta}}^{s}$ commute on $\mathbb{P}[z]$ for all $s>0$ if and only if $T_{f_{m, \delta}}$ and $T_{\Psi_{j}(r) e^{i j \theta}}$ commute on $\mathbb{P}[z]$.

Motivated by this observation and addressing Question (II), we will consider two classes of specific symbols and prove that the algebra generated by Toeplitz operators with symbols in each class is commutative on each Segal-Bargmann space $\mathcal{H}_{s}^{2}\left(\mathbb{C}^{n}\right)$ (the commuting result is uniform in $s$ ). The first is a Banach algebra and refers to symbols of the form $\varphi \xi^{p} \bar{\xi}^{q}$ where $\varphi$ is invariant under a certain action of a product of spheres on $\mathbb{C}^{n}, \xi \in \mathbb{S}^{2 n-1} \subset \mathbb{C}^{n}$ and $p, q \in \mathbb{N}_{0}^{n}$ satisfying some conditions. The second is a $C^{\star}$-algebra and corresponds to symbols $g$ where $g(z)$ depends on the real and on the imaginary part of $z$ in a certain sense. Chapter 3 contains the construction of these algebras together with some other related results that are explained below.

There is an extensive study concerning the commutative algebras generated by Toeplitz operators acting on the (weighted) Bergman spaces $A_{\lambda}^{2}\left(\mathbb{B}^{n}\right)(\lambda>-1$ c.f. Section 1.4) over the unit ball $\mathbb{B}^{n} \subset \mathbb{C}^{n}[22,23,93-97,144,145,168-172]$. A well known example of a commutative $C^{\star}$-algebra generated by Toeplitz operators is induced by the class of bounded measurable radial symbols over $\mathbb{B}^{n}$. This is due to the fact that Toeplitz operators with such symbols are diagonal w.r.t. the standard orthonormal basis of $A_{\lambda}^{2}\left(\mathbb{B}^{n}\right)$. Note that separately radial symbols are precisely those functions which are invariant under a group action of a maximal commutative subgroup of automorphisms $A u t\left(\mathbb{B}^{n}\right)$ of the unit ball (the rotational action of the $n$-torus on $\mathbb{B}^{n}$ ). However, there is a general method for classifying $C^{\star}$-algebras generated by Toeplitz operators which are commmutative on each Bergmann space $A_{\lambda}^{2}\left(\mathbb{B}^{n}\right)$. More precisely, due to a result by R. Quiroga-Barranco and N. Vasilevski [144], the $C^{\star}$-algebra generated by Toeplitz operators with measurable bounded symbols which are invariant under the group action of a maximal commutative subgroup of $A u t\left(\mathbb{B}^{n}\right)$ is commutative on $A_{\lambda}^{2}\left(\mathbb{B}^{n}\right)$ for any $\lambda>-1$. As maximal commutative subgroups of $A u t\left(\mathbb{B}^{n}\right)$ are completely classified [145] in to five classes: quasielliptic, quasi-parabolic, quasi-hyperbolic, nilpotent, and quasi-nilpotent, this gives rise to $n+2$ different cases of commutative $C^{\star}$-algebras. The authors in [144] showed that in each of these cases the corresponding Toeplitz operators are unitary equivalent to a certain multiplication operator and the commutativity result then follows. In the case where $n>1$, it turns out that there are non-geometrically defined symbols which generate commutative Banach algebras of Toeplitz operators. This appears for example for the commutative Banach algebras generated by a class of measurable symbols subordinated to the quasi-elliptic group (c.f. [172]). For the case $n=1$, this commutative algebra is the $C^{\star}$-algebra of Toeplitz operators with bounded radial symbols (note that the commutative Banach algebras of Toeplitz operators on $A_{\lambda}^{2}(\mathbb{D})$ related to 
the quasi-parabolic and to the quasi-hyperbolic groups are also $C^{\star}$-algebras $\left.[22,171]\right)$.

Analogous to the commutative Banach algebras constructed in [172], we will obtain a similar result in the case of the Segal-Bargmann space $\mathcal{H}_{s}^{2}\left(\mathbb{C}^{n}\right)$ (c.f. Section 3.3). Each Banach algebra is generated by Toeplitz operators with symbols in a sub-class of the so-called $k$-quasi homogeneous functions (here $m \in \mathbb{N}$ and $k \in \mathbb{N}^{m}$ are fixed with $|k|=n$ ). The notion of $k$-quasi homogeneous functions is a natural generalization of the quasi-homogeneous functions considered in Chapter 2 in the case of the complex plane (c.f. [130,172, 181]). More precisely, for each tuple $k=\left(k_{1}, \cdots, k_{m}\right)$ the space of $k$-quasi homogeneous functions $\mathcal{H}_{k}$ contains measurable functions of the form $\varphi \xi^{p} \bar{\xi}^{q}$ where $\varphi$ is invariant under the rotational action of $\mathbb{S}^{2 k_{1}-1} \times \cdots \times \mathbb{S}^{2 k_{m}-1}$ on $\mathbb{C}^{n}$ ( $\varphi$ is $k$-quasi radial c.f. (3.1.2)), $\xi \in \mathbb{S}^{2 n-1}$ and $p, q$ are orthogonal multi-indices. For two measurable functions $\varphi \xi^{p} \bar{\xi}^{q}, \psi \xi^{u} \bar{\xi}^{v} \in \mathcal{H}_{k}$ with reasonable growth at infinity, we show that under certain conditions on the indices $p, q, u$ and $v$ the Toeplitz operators $\mathcal{T}_{\varphi \xi^{p} \bar{\xi}^{q}}^{s}$ and $\mathcal{T}_{\psi \xi^{u} \bar{\xi}^{v}}^{s}$ commute on $\mathbb{P}[z]$ for any $s>0$ (Theorem 3.3.1). With these conditions and following the ideas in [172] we are able to construct subspaces $\mathcal{R}_{k}(h) \subset \mathcal{H}_{k} \cap L^{\infty}\left(\mathbb{C}^{n}\right)$ parametrized by tuples $h \in \mathbb{N}_{0}^{m}$ in which the Toeplitz operators with symbols in each $\mathcal{R}_{k}(h)$ commute on $\mathcal{H}_{s}^{2}\left(\mathbb{C}^{n}\right)$ for all $s>0$. We should remark that each symbol space $\mathcal{R}_{k}(h)$ contains the bounded measurable radial symbols and in the case where $n>2$ and $k \neq(1, \cdots, 1)$ the commutative algebras obtained are only Banach and not $C^{\star}$-algebras. When $n=1$ and analogous to the case of the unit ball all these algebras collapse to the $C^{\star}$-algebra generated by Toeplitz operators with bounded radial symbols. We note that in our investigation of these kinds of Banach algebras on the Segal-Bargmann space all the results obtained are analogous to those in [172] for the case of the Bergman space over the unit ball.

There is a more general notion of Toeplitz operators acting on a domain in $\mathcal{H}_{s}^{2}\left(\mathbb{C}^{n}\right)$ with symbols having a suitable growth at infinity as introduced in $[14,16,117,118]$. In Section 3.2, we employ a natural extension of the notion of Toeplitz operators acting on $\mathbb{P}[z]$ with symbols in a reasonable sub-class of $k$-quasi radial functions containing all $\mu_{(s)}$-square integrable radial functions. We should note that in our generalized notion of Toeplitz operators with $k$-quasi radial symbols the operators are still diagonal w.r.t the standard orthonormal basis. We aim to construct a $C^{\star}$-algebra formed only by Toeplitz operators with radial symbols in this general notion (note that the product of two Toeplitz operators is rarely a Toeplitz operator again). For this reason we will consider a suitable $C^{\star}$-algebra $\mathcal{A}_{1}\left(\mathbb{C}^{n}\right)$ of bounded diagonal operators on $\mathbb{P}[z]$. By studying some Stieltjes moment problems we show that for any $s>0$ and any $T \in \mathcal{A}_{1}\left(\mathbb{C}^{n}\right)$ there is a radial function so that the Toeplitz operator with this symbol acting on $\mathcal{H}_{s}^{2}\left(\mathbb{C}^{n}\right)$ coincide with $T$ on $\mathbb{P}[z]$. Consequently, we obtain a $C^{\star}$-algebra containing only Toeplitz operators and which is commutative on each $\mathcal{H}_{s}^{2}\left(\mathbb{C}^{n}\right)$ for any $s>0$ (Corollary 3.2.1). We want to remark that this result is a generalization of the particular case $n=s=1$ considered in [95]. Moreover, in our investigation for the general case of $k$-quasi radial functions (not necessarily radial), we are able to find a subclass of $k$-quasi radial functions for which the $C^{\star}$-algebra generated by Toeplitz operators with symbols in this class is commutative on each $\mathcal{H}_{s}^{2}\left(\mathbb{C}^{n}\right)$ and contains only Toeplitz operators (c.f. Corollary 3.2.2). 
In [167], N. Vasilevski proved that there is an infinite direct sum decomposition of $L^{2}\left(\mathbb{C}^{n}\right):=L^{2}\left(\mathbb{C}^{n}, d \mu_{(1)}\right)$ into spaces $F_{(k)}^{2}\left(\mathbb{C}^{n}\right)$ of entire (holomorphic) type functions. These spaces are parametrized through all multi-indices $k=\left(k_{1}, \cdots, k_{n}\right) \in \mathbb{N}^{n}$ and they are called the true- $k$-Fock spaces (c.f. Section 3.4). We will construct an isomorphism between each $F_{(k)}^{2}\left(\mathbb{C}^{n}\right)$ and the Lebesgue space $L^{2}\left(\mathbb{R}^{n}\right):=L^{2}\left(\mathbb{R}^{n}, d v\right)$ over $\mathbb{R}^{n}$ where $v$ is the usual Lebesgue measure (c.f. [167] for the case $n=1$, and Theorem 3.4.2 for arbitrary $n \in \mathbb{N}$ ). For each fixed tuple $k$ and via the orthogonal projection $P_{(k)}$ from $L^{2}\left(\mathbb{C}^{n}\right)$ onto $F_{(k)}^{2}\left(\mathbb{C}^{n}\right)$ we introduce the notion of true$k$-Toeplitz operators $T_{g}^{(k)}$ with bounded measurable symbols $g$ as $T_{g}^{(k)}=P_{(k)} M_{g}$. We note that for $k=(1, \cdots, 1) \in \mathbb{N}^{n}$ the space $F_{(k)}^{2}\left(\mathbb{C}^{n}\right)$ is the Segal-Bargmann space $H^{2}\left(\mathbb{C}^{n}\right):=\mathcal{H}_{1}^{2}\left(\mathbb{C}^{n}\right)$ and $T_{g}^{(k)}=\mathcal{T}_{g}^{1}$ is the usual Toeplitz operator with symbol $g$ acting on $H^{2}\left(\mathbb{C}^{n}\right)$. This shows that true- $k$-Toeplitz operators acting on the true- $k$-Fock spaces are generalizations of Toeplitz operators acting on $H^{2}\left(\mathbb{C}^{n}\right)$.

We are interested in a symbol class for which the $C^{\star}$-algebra generated by true- $k$-Toeplitz operators is commutative on $F_{(k)}^{2}\left(\mathbb{C}^{n}\right)$ for any $k \in \mathbb{N}^{n}$. For this reason, we consider bounded symbols of the form $\Psi(z)=a(A(x)) e^{i u \cdot y}$ where $x, y \in \mathbb{R}^{n}$ with $z=x+i y, A$ is an endomorphism of $\mathbb{R}^{n}$ and $u \in \operatorname{ker} A=\mathcal{H}$. Similar to a result in [167] we prove that $T_{\Psi}^{(k)}$ is unitary equivalent to an operator on $L^{2}\left(\mathbb{R}^{n}\right)$ which is a composition of a shift and a multiplication operator. Using this fact we show that for a fixed subspace $\mathcal{H} \subseteq \mathbb{R}^{n}$ the $C^{\star}$-algebra generated by true- $k$-Toeplitz operators (respectively Toeplitz operators) with symbols of the above form is commutative on $F_{(k)}^{2}\left(\mathbb{C}^{n}\right)$ (respectively on each Segal-Bargmann space $\mathcal{H}_{s}^{2}\left(\mathbb{C}^{n}\right)$ ).

At the end of Chapter 3, we use the fact that the Segal-Bargmann space $\mathcal{H}_{s}^{2}\left(\mathbb{C}^{n}\right)$ over $\mathbb{C}^{n}$ is a tensor product of Segal-Bargmann spaces over lower dimensional complex spaces to combine our results for the commutative Banach and $C^{\star}$-algebras indicated above. As a consequence, we will obtain a more general type of Banach algebras generated by Toeplitz operators which is commutative on each $\mathcal{H}_{s}^{2}\left(\mathbb{C}^{n}\right)$ for any $s>0$.

In Chapter 4 we apply Toeplitz operator theory techniques to the computation of heat kernels for a class of elliptic and sub-elliptic differential operators. For this reason, we introduce (via the Bargmann transform from $L^{2}\left(\mathbb{R}^{n}\right)$ onto the Segal-Bargmann space $\left.H^{2}\left(\mathbb{C}^{n}\right)\right)$ the notion of the „heat kernel of a Toeplitz operator" (c.f. Definition 4.3.3 for a precise statement for the notion of the heat kernel of a Toeplitz operator). Introduced by V. Bargmann in [12], the Bargmann transform $\beta_{\sqrt{2}}$ maps $L^{2}\left(\mathbb{R}^{n}\right)$ isometrically onto $H^{2}\left(\mathbb{C}^{n}\right)$. Via the push-forwards under $\beta_{\sqrt{2}}$ each operator on $L^{2}\left(\mathbb{R}^{n}\right)$ can be regarded as an operator acting on $H^{2}\left(\mathbb{C}^{n}\right)$. In the case where the operator symbol $g$ is a polynomial over $\mathbb{C}^{n}$ in the complex variables $z$ and $\bar{z}$ the Toeplitz operator $T_{g}$ corresponds to a partial differential operator with polynomial coefficient acting on a suitable dense domain in $L^{2}\left(\mathbb{R}^{n}\right)$. The Bargmann transform also gives a one-to-one correspondence between a subclass of integral operators acting on $H^{2}\left(\mathbb{C}^{n}\right)$ and another subclass of integral operators on $L^{2}\left(\mathbb{R}^{n}\right)$. The kernels on both sides are related via an application of the Bargmann transform (c.f. Proposition 4.2.2).

Assume that we are given a differential operator $\mathcal{L}$ with polynomial coefficients on $L^{2}\left(\mathbb{R}^{n}\right)$ corresponding to a Toeplitz operator $T_{g}$ on $H^{2}\left(\mathbb{C}^{n}\right)$ such that the ,heat kernel” of $T_{g}$ (c.f. Def- 
inition 4.3.3) exists and defines an integral operator in the above mentioned subclass. Then the heat kernel of $\mathcal{L}$ is given by an application of the inverse Bargmann transform to the ,heat kernel" of $T_{g}$. Motivated by this observation, in Chapter 4 we are interested in investigating the ,heat kernel" of a certain type of Toeplitz operators with polynomial symbols acting on $H^{2}\left(\mathbb{C}^{n}\right)$. Using the Bargmann transform we deduce the heat kernel of a class of elliptic operators acting on $L^{2}\left(\mathbb{R}^{n}\right)$. As the simplest example we obtain the well-known heat kernel of the Hermite operator on $\mathbb{R}^{n}$ as well as that of the isotropic twisted Laplacian on $\mathbb{R}^{2 n}$ (c.f. for example [56, 161, 162]). As an application and using the partial Fourier transformation we obtain the heat kernel of the Grusin operator on $\mathbb{R}^{n+1}$ as well as that of the sub-Laplace operator on the $(2 n+1)$-dimensional Heisenberg group.

In Chapter 4 we combine results due to F.A. Berezin [29] and J. Janas [118] to obtain the ,heat kernel" of a certain class of Toeplitz operators acting on a dense domain of $H^{2}\left(\mathbb{C}^{n}\right)$. On the one hand, Berezin's result states that if $T_{f}$ is a selfadjoint operator with a positive measurable symbol $f$ then the exponential operator $e^{-s T_{f}}$ on $H^{2}\left(\mathbb{C}^{n}\right)$ is given by $e^{-s T_{f}}=$ $\lim _{N \longrightarrow \infty}\left(T_{e^{-\frac{s}{N} f}}\right)^{N}$ where convergence is understood in the strong sense. On the other hand, J. Janas proved that if $f$ is a $t$-radially symmetric function (c.f. Section 4.3 for the definition) such that the space of holomorphic polynomial $\mathbb{P}[z]$ over $\mathbb{C}^{n}$ is contained in the domain of $T_{f}$ then $T_{f}$ is essentially selfadjoint as an operator on the dense domain $\mathbb{P}[z]$. In this case we will not distinguish between $T_{f}$ and its unique self adjoint extension. Combining these two results we can therefore apply Berezin's result for Toeplitz operators with positive $t$-radially symmetric polynomial symbols. Note that Berezin's result expresses the kernel of the exponential operator $e^{-s T_{f}}$ as an infinite limit of multiple integrals over $\mathbb{C}^{n N}(N \longrightarrow \infty)$. However, in some cases each multiple integral can be reduced to a simple integral over $\mathbb{C}^{n}$. In particular, this is the case when the higher products of Toeplitz operators $\left(T_{e^{-\frac{s}{N}} f}\right)^{N}$ is again a Toeplitz operator. The core part of Chapter 4 is devoted to the following problem

(P2) Consider a positive semidefinite matrix $A \in M_{n}(\mathbb{C})$ such that $(A+\bar{A})$ is positive definite and define $f(z)=z A \bar{z}$, where $z, \bar{z} \in \mathbb{C}^{n}$. Let $\mathcal{L}_{1}$ be the partial differential operator acting on a suitable dense domain in $L^{2}\left(\mathbb{R}^{n}\right)$ with polynomial coefficients corresponding to the Toeplitz operator $T_{f}$. Then

(a) What is the ,heat kernel" of $T_{f}$ on $H^{2}\left(\mathbb{C}^{n}\right)$ ?

(b) Let $\bar{A} \in M_{n}(\mathbb{C})$ denotes the matrix whose entries are the conjugate entries of $A$ i.e. $\bar{A}:=\operatorname{Re}(A)-i \operatorname{Im}(A)$ where $\operatorname{Re}(A)$ and $\operatorname{Im}(A)$ denotes the real and the imaginary part of $A$, respectively. Assume that $A$ and $\bar{A}$ commute. What is the heat kernel of $\mathcal{L}_{1}$ on $L^{2}\left(\mathbb{R}^{n}\right)$ ?

As a first step we check that $f$ is $t$-radially symmetric and we employ Berezin's result stated above. We will show that $e^{-s T_{f}}$ is also a Toeplitz operator acting on a dense domain in $H^{2}\left(\mathbb{C}^{n}\right)$. We then obtain the „heat kernel” of $T_{f}$ by a simple expression involving the exponential operator $e^{-s T_{f}}$ and the reproducing kernel of $H^{2}\left(\mathbb{C}^{n}\right)$ (c.f. Theorem 4.3.5). Moreover, we show that the operator $\mathcal{L}_{1}$ is elliptic, semibounded below and in the case where $A$ and $\bar{A}$ commute its heat 
kernel is obtained explicitly by a suitable application of the inverse Bargmann transform to the „heat kernel” of $T_{f}$ (c.f. Corollary 4.3.2).

In our investigation of the ,heat kernel” for Toeplitz operators, we were able in some cases to use the method of partial Fourier transform to obtain the heat kernel of certain sub-elliptic positive essentially selfadjoint differential operators with polynomial coefficients on $L^{2}\left(\mathbb{R}^{m}\right)$ where $m \geq 2$. We write $m=n+1$ and apply the partial Fourier transform w.r.t. the last variable in order to eliminate this variable and to obtain a one parameter family of elliptic operators corresponding to the previously mentioned situation on $L^{2}\left(\mathbb{R}^{n}\right)$. For this reason, we start Chapter 4 by introducing a family of Bargmann transforms $\beta_{t}$ parametrized by $t>0$. This is essential as this parameter will play the role of the eliminated variable. In fact, we will consider a certain type of differential operators having a specific form (4.3.7) with polynomial coefficients involving the entries of the above matrix $A$. Even in the case where $A$ is complex, we will express each examined operator $\mathcal{L}$ as a sum of squares of real valued vector fields. It turns out that these vector fields together with their higher commutators span the tangent space of $\mathbb{R}^{m}$ at every point i.e. they satisfy the ,bracket generating condition” turning $\mathcal{L}$ into a subelliptic operator [107]. Moreover, we will prove that $\mathcal{L}$ is symmetric, positive and essentially selfadjoint on the Schwartz space $\mathcal{S}\left(\mathbb{R}^{n+1}\right)$ (c.f. Theorems 4.3.1 and 4.3.7). In order to obtain the heat kernel of $\mathcal{L}$, we apply the partial Fourier transformation at each fixed point $\xi \neq 0$ to obtain an elliptic partial differential operator $\mathcal{L}_{\xi}$ on $L^{2}\left(\mathbb{R}^{n}\right)$. Via the Bargmann transform $\beta_{t}$ with $t=\sqrt{2|\xi|}$, the operator $\mathcal{L}_{\xi}$ is unitary equivalent to a Toeplitz operator $\alpha T_{z A \bar{z}}$ where $\alpha$ is a constant depending on $\xi$ and $A$ is a matrix satisfying the conditions in (P2) and whose entries are independent of $\xi$. Therefore, the heat kernel $k_{\xi}(x, y) \in C^{\infty}\left(\mathbb{R}^{n} \times \mathbb{R}^{n}\right)$ of $\mathcal{L}_{\xi}$ is obtained by the method explained above. It turns out that $k(\xi, x, y):=k_{\xi}(x, y)$ is also smooth in $\xi$. Under certain assumptions on $k(\xi, x, y)$, the heat kernel of $\mathcal{L}$ is then obtained by an application of the inverse partial Fourier transform to the function $k(\xi, x, y)$. To illustrate the importance of our results we will recover the well-known integral formula for the heat kernel of the sub-Laplace operator on the $(2 n+1)$-dimensional Heisenberg group as well as that of the Grusin operator on $\mathbb{R}^{n+1}[56]$.

Chapter 5 of this thesis is devoted to study the uniform compactness (w.r.t. the standard weight parameter) of a Toeplitz operator with a fixed symbol acting on the standard weighted Bergman spaces over a bounded symmetric domain. Such a problem has been considered by W. Bauer, L.A. Coburn and J. Isralowitz in [15] for the case of Toeplitz operators with symbols of bounded mean oscillation acting on the Segal-Bargmann spaces. The case of Toeplitz operators (also with symbol of bounded mean oscillation on the unit ball) acting on the standard weighted Bergman spaces over the unit ball was also investigated in [15]. The authors of [15] proved that if $g \in B M O^{1}\left(\mathbb{C}^{n}\right)$ the Toeplitz operator $\mathcal{T}_{g}^{t_{0}}$ is compact on $\mathcal{H}_{t_{0}}^{2}\left(\mathbb{C}^{n}\right)$ for some $t_{0}>0$ if and only if $\mathcal{T}_{g}^{t}$ is compact on $\mathcal{H}_{t}^{2}\left(\mathbb{C}^{n}\right)$ for all $t>0$. This result remains valid for Toeplitz operators $T_{g}^{\lambda}$ acting on the weighted Bergman space $A_{\lambda}^{2}\left(\mathbb{B}^{n}\right)$ (here $\lambda \in(-1, \infty)$ ) whenever $g \in B M O^{1}\left(\mathbb{B}^{n}\right)$. We refer the reader to Sections 1.3 and 1.4 were a detailed description for the methods used in [15] is given. 
Bounded symmetric domains are the natural generalization of the unit ball $\mathbb{B}^{n} \subset \mathbb{C}^{n}$, and are Hermitian symmetric spaces when endowed with the Bergman metric. Irreducible bounded symmetric domains have been completely classified by E. Cartan [57] into four classical domains and two exceptional ones. Up to a biholomorphic equivalence each irreducible bounded symmetric domain $\Omega \subset \mathbb{C}^{n}$ is uniquely determined by a triple of non-negative integers $(r, a, b)$ called the type of $\Omega$. It is well known that to each $\Omega$ there is attached a unique polynomial $h(z, w)\left(z, w \in \mathbb{C}^{n}\right)$ called the Jordan triple determinant. The real valued function $h(z, z)$ defines standard normalized weights $\mu_{\nu}$ on $\Omega$ parametrized by $\nu \in \mathbb{R}$ such that $\nu>p-1$, where $p$ is the genus of $\Omega$ depending on $(r, a, b)$ and given by (5.2.1). For each $\nu>p-1$ and for a measurable function $g$ on $\Omega$ we denote by $T_{g}^{\nu}$ the Toeplitz operator with symbol $g$ acting on the Bergman space $H_{\nu}^{2}:=H^{2}\left(\Omega, d \mu_{\nu}\right)$. A natural question in the study of the Toeplitz operators is whether the compactness of $T_{g}^{\nu}$ is uniform w.r.t. the weight parameter $\nu$. Under the assumption that $g \in L^{\infty}(\Omega)$ we will show that the compactness of $T_{g}^{\nu}$ is independent of $\nu$, for $\nu$ large enough. On the one hand, under the above assumption our result is a generalization to that in [15] since we deal with a wider class of domains. On the other hand, our result is somewhat limited since only bounded symbols are considered. The main ingredients in our investigation for the uniform compactness of the Toeplitz operator $T_{g}^{\nu}$ where $g \in L^{\infty}(\Omega)$ are the following

(a) An upper estimate for the sup-norm of the Berezin transform $\tilde{g}_{\nu_{0}}$ in terms of the operator norm of $T_{g}^{\nu}$ whenever $\nu$ and $\nu_{0}$ are suitable weights.

(b) A density result for a class of Toeplitz operators in the space of all compact operators on $H_{\nu}^{2}$.

(c) Engliš's result [77] for the equivalence between the compactness of Toeplitz operators with bounded symbols and the vanishing of the Berezin transform of the symbol near the boundary of $\Omega$.

For each pair $\left(\nu, \nu_{0}\right)$ of weights we construct a trace class operator on $H_{\nu}^{2}$ such that $\tilde{g}_{\nu_{0}}$ is given as the operator trace. We use then a standard estimate for the trace norm to obtain (a). This result is valid not only for bounded functions but also for wider class of functions. As for (b), we will prove the density result for all Bergman spaces. Namely, we show that the space of Toeplitz operators with symbols that are continuous with compact support on the domain is norm dense in the space of all compact operators acting on the Bergman space over the domain. In particular, this holds true for the case of the Bergman space $H_{\nu}^{2}$. We also use a result in [77] which states that $T_{g}^{\nu}$ is compact on $H_{\nu}^{2}$ if and only if $\tilde{g}_{\nu}$ vanishes on $\partial \Omega$, for $\nu$ large enough. By combining these results we prove that the compactness of $T_{g}^{\nu}$ is independent of the weight parameter $\nu$, for $\nu$ large enough. Finally, we want to remark that it is still not clear whether our result can be extended to the case of Toeplitz operators with symbols having a bounded mean oscillation on $\Omega$ where such a result has been obtained for the case $\Omega=\mathbb{B}^{n}$ [15]. This is due to the fact that the equivalence between the vanishing of the Berezin transform on $\partial \Omega$ and the compactness of Toeplitz operators is not clear for such a space of symbols on a general bounded symmetric domain. 
We will now state some of our main results obtained in this thesis. In the following we only provide two results addressed to each of the above mentioned problems.

\section{Main results:}

For the commuting problem (P1) of Toeplitz operators with quasi-homogeneous symbols acting on the Segal-Bargmann space over the complex plane we indicate the following results

Theorem 1. Let $\Psi$ be a measurable complex valued function on $\mathbb{C}$ of polynomial growth at infinity and write $\Psi\left(r e^{i \theta}\right)=\sum_{j=-\infty}^{\infty} \Psi_{j}(r) e^{i j \theta}$ as an expansion in $L^{2}(\mathbb{C}, d \mu)$. Let $f_{m, \delta}\left(r e^{i \theta}\right)=$ $z^{l} \bar{z}^{k}=r^{m} e^{i \delta \theta}$ be a monomial where $m=l+k \in \mathbb{N}$ and $\delta=l-k \in \mathbb{N}_{0}$. For each $j>\delta$, we define a holomorphic function $G_{j}(z)$ for $\operatorname{Re}(z)>-j-1$ by

$$
G_{j}(z):=\prod_{l=1}^{j} \Gamma\left(\frac{z+p+l}{\delta}\right) \prod_{l=1}^{j-\delta}\left[\Gamma\left(\frac{z+\delta+l}{\delta}\right)\right]^{-1} \delta^{z},
$$

where $p:=\frac{\delta+m}{2}$. Suppose that $T_{f_{m, \delta}} T_{\Psi}=T_{\Psi} T_{f_{m, \delta}}$. Then for each $j>\delta$ there exists $a$ trigonometric polynomial $p(z)=\sum_{|l|<\frac{\delta}{4}} a_{l} e^{\frac{2 \pi i l z}{\delta}}$ such that

$$
\Psi_{j}(r)=2 M^{-1}\left[G_{j}(z) p(z)\right]\left(r^{2}\right) r^{-j-2} e^{r^{2}},
$$

where $M^{-1}$ denotes the inverse Mellin transform.

Theorem 2. Let $f_{m, \delta}\left(r e^{i \theta}\right)=r^{m} e^{i \delta \theta}$ be a monomial, and let $l \in \mathbb{Z}$ such that $|l|<\frac{\delta}{2 \pi} \arccos \frac{3}{4}$. Then for each $j>\delta$ the function

$$
\varphi_{j}(r) e^{i j \theta}:=M^{-1}\left[G_{j}(z) e^{\frac{2 \pi i l z}{\delta}}\right]\left(r^{2}\right) r^{-j-2} e^{r^{2}} e^{i j \theta},
$$

defines an operator symbol such that the commutator $\left[T_{f_{m, \delta}}, T_{\varphi_{j} e^{i j \theta}}\right]$ is well defined and vanishing on the space of holomorphic polynomials. Moreover, $\varphi_{j}(r) e^{i j \theta}$ is of polynomial growth at infinity in case $l=0$.

For the commutative algebras generated by Toeplitz operators acting on Segal-Bargmann space $\mathcal{H}_{s}^{2}\left(\mathbb{C}^{n}\right)$ we have

Theorem 3. Let $k=\left(k_{1}, \cdots, k_{m}\right)$ be a tuple of positive integers such that $k_{1}+\cdots+k_{m}=n$. Without loss of generality assume that $k_{1} \leqslant k_{2} \leqslant \cdots \leqslant k_{m}$. Consider a tuple $h=\left(h_{1}, \cdots, h_{m}\right)$ with $h_{j}=0$ if $k_{j}=1$ and $1 \leqslant h_{j} \leqslant k_{j}-1$. In the case $k_{j_{0}}=k_{j_{1}}$ with $j_{0}<j_{1}$ put $h_{j_{0}} \leqslant h_{j_{1}}$. Let $\mathcal{R}_{k}(h)$ be the space of all bounded $k$-quasi-homogeneous symbols

$$
\varphi\left(r_{1}, \cdots, r_{m}\right) \xi^{p} \bar{\xi}^{q}
$$

where $p, q$ are multi-indices of the form (3.3.6) and satisfying (3.3.7). Then the Toeplitz operators with symbols in $\mathcal{R}_{k}(h)$ generate a commutative Banach algebra in $\mathcal{L}\left(\mathcal{H}_{s}^{2}\right)$ for any $s>0$. Moreover, for $n>2$ and $k \neq(1, \cdots, 1)$ these algebras are not $C^{\star}$-algebras. 
Theorem 4. If $\mathcal{H}$ is a linear subspace of $\mathbb{R}^{n}$ then the $C^{\star}$-algebra generated by the set

$$
\left\{\mathcal{T}_{a(A(x)) e^{i u \cdot y}}^{s} \mid a \in L^{\infty}\left(\mathbb{R}^{n}\right), A \text { is an endomorphism } \mathbb{R}^{n} \text { with ker } A=\mathcal{H} \text { and } u \in \mathcal{H}\right\}
$$

is commutative on $\mathcal{H}_{s}^{2}$. Moreover, for each $k \in \mathbb{N}^{n}$ this result holds true for the $C^{\star}$-algebra generated by true- $k$-Toeplitz operators $T_{a(A(x)) e^{i u \cdot y}}^{(k)}$ acting on the true-k-Fock space $F_{(k)}^{2}\left(\mathbb{C}^{n}\right)$.

Addressing the Problem (P2) for the ,heat kernel" of Toeplitz operators we mention the following results

Theorem 5. Let A be a $n \times n$ positive semidefinite matrix. Then the ,heat kernel" of the Toeplitz operator $T_{z A \bar{z}}$ on $H^{2}\left(\mathbb{C}^{n}\right)$ is given by

$$
K_{A}(s, z, w)=e^{-s \operatorname{tr}(A)} e^{z e^{-s A} \bar{w}}, \quad(s, z, w) \in \mathbb{R}_{+} \times \mathbb{C}^{n} \times \mathbb{C}^{n} .
$$

Theorem 6. Let $A=\left(a_{j k}\right)$ be an $n \times n$ positive semidefinite matrix. For each $\xi \neq 0$ consider the differential operator $\mathcal{L}_{\xi}$ on $\mathbb{R}^{n}$ defined by

$$
\mathcal{L}_{\xi}=\sum a_{j j}\left(\xi^{2} x_{j}^{2}-\frac{\partial^{2}}{\partial x_{j}^{2}}\right)+\sum_{j \neq k} a_{j k}\left(\xi x_{k}+\frac{\partial}{\partial x_{k}}\right)\left(\xi x_{j}-\frac{\partial}{\partial x_{j}}\right) .
$$

Then $\mathcal{L}_{\xi}$ is essentially selfadjoint and semibounded below. Moreover, if $(A+\bar{A})$ is positive definite then $\mathcal{L}_{\xi}$ is elliptic. If we assume further that the matrices $A$ and $\bar{A}$ commute then the heat kernel of $\mathcal{L}_{\xi}$ denoted by $k_{\xi}(s, x, y)$ and defined on $\mathbb{R}_{+} \times \mathbb{R}^{n} \times \mathbb{R}^{n}$ is given by

$$
\begin{aligned}
k_{\xi}(s, x, y)= & e^{-s|\xi| \operatorname{tr}(A)}\left(\frac{|\xi|}{\pi}\right)^{\frac{n}{2}} \sqrt{\operatorname{det}\left(I d-e^{-2 s|\xi|(A+\bar{A})}\right)^{-1}} \\
& \times \exp \left\{-\frac{|\xi|}{2} y\left(I d-e^{-2 s|\xi|(A+\bar{A})}\right)^{-1}\left(I d+e^{-2 s|\xi|(A+\bar{A})}\right) y\right. \\
& -\frac{|\xi|}{2} x\left(I d-e^{-2 s|\xi|(A+\bar{A})}\right)^{-1}\left(I d+e^{-2 s|\xi|(A+\bar{A})}\right) x \\
& \left.+2|\xi| y\left(I d-e^{-2 s|\xi|(A+\bar{A})}\right)^{-1} e^{-2 s|\xi| A} x\right\} .
\end{aligned}
$$

As for the problem of the uniform compactness of Toeplitz operators with bounded symbols we fix an irreducible bounded symmetric domain $\Omega \subset \mathbb{C}^{d}$ of multiplicities $a$ and $b$, rank $r$ and genus $p$. For $\nu>p-1$ we consider the space of symbols

$$
\tau_{\nu}(\Omega):=\left\{g: \Omega \longrightarrow \mathbb{C} \mid g K_{\nu,[a]} \in L^{2}\left(\Omega, d \mu_{\nu}\right) \text { for all } a \in \Omega\right\},
$$

where $K_{\nu,[a]}$ is the reproducing kernel of $H_{\nu}^{2}$ at the point $a \in \Omega$.

Theorem 7. Let $\nu>p-1, \nu_{0}>d$ with $\left|\nu-\nu_{0}\right|>\frac{r-1}{2}$ a and write $\tilde{\nu}=\min \left\{\nu, \nu_{0}\right\}$. Then there exists $C\left(\nu, \nu_{0}\right)>0$ such that for all $g \in \tau_{\nu}(\Omega) \cap L^{1}\left(\Omega, d \mu_{\tilde{\nu}}\right)($ c.f. (5.2.5)) we have

$$
\left\|\widetilde{g}_{\nu_{0}}\right\|_{\infty} \leq C\left(\nu, \nu_{0}\right)\left\|T_{g}^{\nu}\right\|
$$

Theorem 8. Let $\Omega \subset \mathbb{C}^{d}$ be an irreducible bounded symmetric domain and suppose that

$$
\nu, \nu_{0}>\max \left\{d, p-1+\frac{r-1}{4} a+\sqrt{\left(\frac{r-1}{4} a\right)^{2}+\frac{r-1}{2} a\left(\frac{r-1}{2} a+p-1\right)}\right\} .
$$

Then for any $g \in L^{\infty}(\Omega)$ we have the equivalence:

$$
T_{g}^{\nu_{0}} \text { is compact on } H_{\nu_{0}}^{2} \text { if and only if } T_{g}^{\nu} \text { is compact on } H_{\nu}^{2} \text {. }
$$




\section{Organization of the text:}

CHAPTER 1: This is an introductory chapter and most of the results here can be found in the literature. In this chapter we set notation and terminology and we collect some basic tools which will be used later in our analysis. We also explain some recent results in the area of the analysis of Toeplitz operators which motivate our work. As Bergman spaces are examples of reproducing kernel Hilbert spaces we prefer to start this chapter by introducing the general theory of reproducing kernel Hilbert spaces. We show that the Berezin transform is one-toone on a class of bounded operators, which will be used in Chapter 2 for reducing problem (P0) to (P1). In Section 1.2 we consider Bergman spaces (w.r.t. a suitable finite measure) over a domain $\Omega \subseteq \mathbb{C}^{n}$ and indicate some useful properties of Toeplitz operators acting on these spaces. We generalize the density result in [35] from the case of the Segal-Bargmann space to all Bergman spaces. This compactness criteria is essential in our investigation for the uniform compactness of Toeplitz operators with bounded symbols acting on the standard weighted Bergman spaces over a bounded symmetric domain (c.f. Chapter 5). Sections 1.3 and 1.4 are devoted to introduce the Segal-Bargmann and the (weighted) Bergman spaces over the unit ball, respectively. We explain some properties of Toeplitz operators acting on such spaces and their connection to the Berezin transform of the symbol. In particular, we give a detailed explanation of the method used in [15] for investigating of the uniform compactness of a Toeplitz operator with symbols having a bounded mean oscillation acting on the SegalBargmann spaces or the weighted Bergman spaces over the unit ball. The results collected in Section 1.4 will help us later in comparing our results to those in $[15,35,66,95,129,172]$

CHAPTER 2: We investigate the commuting problem of Toeplitz operators with quasihomogeneous symbols (P0). Section 2.1 is devoted to a detailed description of (P0) as well as a brief explanation of the methods used for solving it. We also state the main result in [66] for the problem which motivated (P0). This enables us throughout the chapter to compare our results for the case of the Segal-Bargmann space to those in [66] for the case of the Bergman space over the unit disc. In Section 2.2, we construct a space of functions $\mathcal{E} \subset L^{2}$ for which Toeplitz operators with symbols in $\mathcal{E}$ are densely defined on a domain in $H^{2}$ containing $\mathbb{P}[z]$. Moreover, we represent each function in $\Psi \in \mathcal{E}$ as an $L^{2}$-series expansion of quasi-homogeneous functions $\Psi\left(r e^{i \theta}\right)=\sum_{j=-\infty}^{\infty} \Psi_{j}(r) e^{i j \theta}$. The problem (P0) is then reduced to the case $\delta \geq 0$. Finally, we introduce the concept of the Mellin transform of a function which will be a useful tool in our investigation. We start Section 2.3 by proving that if $f$ is a measurable function with at most polynomial growth at infinity and $\Psi \in \mathcal{E}$ then $T_{f}$ and $T_{\Psi}$ commute on $\mathbb{P}[z]$ if and only if for each $j \in \mathbb{Z}$ the Toeplitz operators $T_{f}$ and $T_{\Psi_{j} e^{i j \theta}}$ commute on $\mathbb{P}[z]$. As a consequence (P0) is reduced to (P1). Furthermore, for each fixed $j \in \mathbb{Z}$ we show that the commuting problem in (P1) can be described by certain equivalent conditions involving the Mellin transform of $\Psi_{j}$ at specific points. In order to characterize the functions $\left\{\Psi_{j}\right\}_{j \in \mathbb{Z}}$ we decompose the problem into three parts: (1) case $j>\delta$ (2), case $j<0$, (3) $0 \leqslant j \leqslant \delta$. Section 2.4 treats case (1) and 
Theorems 1 and 2 are proved. We show that for an infinite number of indices $j$ there is only one (up to a constant factor) function $\Psi_{j}$ of polynomial growth solving (P1). Example 2.4.1 treats the case $\delta=0$ showing that if the monomial is non-constant then $\Psi$ is radial. Sections 2.5 and 2.6 deals with the case (2) and (3), respectively. In these two sections results analogous to Theorems 1 and 2 are obtained. In Section 2.7 some applications of our results are indicated (c.f. the explanation above). Finally, we state two conjectures which are inspired by the results obtained in this chapter.

CHAPTER 3: This chapter is devoted to the construction of the above mentioned types of commutative algebras generated by Toeplitz operators. In Section 3.1 we introduce the concept of $k$-quasi-radial and $k$-quasi-homogeneous functions. We then explain the contents and the main results in this chapter. In Section 3.2 we give a more general notion of Toeplitz operators with $k$-quasi-radial symbols. By studying suitable Stieltjes moment problems we construct commutative $C^{\star}$-algebras containing only Toeplitz operators with radial symbols in the previously mentioned sense. Moreover, for the case of arbitrary $k$-quasi-radial functions we are able to obtain commutative $C^{\star}$-algebras of Toeplitz operators whose symbols are in a sub-class of the $k$-quasi-radial functions. Section 3.3 deals with the commutative Banach algebras generated by Toeplitz operators with k-quasi-homogeneous symbols. We search for sufficient conditions on two $k$-quasi-homogeneous functions so that the Toeplitz operators with the these symbols commute on each Segal-Bargmann space $H_{s}^{2}$. This enables us to give a proof of Theorem 3. In Section 3.4 we are interested in obtaining the commutative $C^{\star}$-algebras as described in Theorem 4. We start by introducing the true- $k$-Fock spaces as well as the notion of the true- $k$-Toeplitz operators, with suitable symbols, acting on these spaces. We show that each true- $k$-Toeplitz operator, with symbols of the form considered in Theorem 4, is unitary equivalent to an operator on $L^{2}\left(\mathbb{R}^{n}\right)$ which is a composition of a shift and a multiplication operator. This leads to a construction of commutative $C^{\star}$-algebras generated by true- $k$-Toeplitz operators. Using this fact together with Remark 3.4.1 for the case of Toeplitz operators acting on the Segal-Bargmann spaces, Theorem 4 then follows. Finally, we combine the commutative algebras obtained in the last two sections to form a more general type of commutative Banach algebras of Toeplitz operators. Motivated by the results obtained, we end this chapter by indicating some open problems.

CHAPTER 4: We use Toeplitz operator theory techniques to obtain the heat kernel of a class of elliptic bounded below essentially self-adjoint differential operators. Together with the partial Fourier transformation method we give an explicit integral kernel for a sub-class of sub-elliptic partial differential operators. In Section 4.1 we explain how we will combine the Bargmann transform, Berezin's result [29], and the partial Fourier transformation method to obtain the heat kernel of these operators. Section 4.2 is devoted to the concept of the Bargmann transform. Namely, we will introduce a family of Bargmann transforms $\beta_{t}$ parametrized by $t>0$. Via the push-forward under $\beta_{t}$ we consider a class of integral operators acting on a dense domain of $H^{2}\left(\mathbb{C}^{n}\right)$ and exhibit their corresponding operators acting on a dense domain 
of $L^{2}\left(\mathbb{R}^{n}\right)$. We also calculate the ,inverse Bargmann transform" of $e^{z A w}$ where $z, w \in \mathbb{C}^{n}$ and $A \in M_{n}(\mathbb{C})$ is Hermitian satisfying some conditions. This result will be used later to obtain the heat kernel of the considered elliptic operators. Section 4.3 is the core part of Chapter 4. We start by explaining the method of the partial Fourier transform for obtaining the heat kernel of a certain class of differential operators. Next, we consider a class of differential operators with polynomial coefficient and show that they are sub-elliptic positive and essentially selfadjoint. Applying the partial Fourier transform on these operators we obtain a class of elliptic essentially self-adjoint bounded below differential operators. We then investigate the ,heat kernel" of the corresponding Toeplitz operators on $H^{2}\left(\mathbb{C}^{n}\right)$ and Theorems 5 and 6 are proved. Using the Bargmann transform and the result in the previous section we obtain the heat kernel of the above mentioned elliptic operators. Under certain condition on the heat kernel of these operators we are able to obtain the heat kernel for a class of sub-elliptic operators. Moreover, several examples which illustrate the importance of the considered class of differential operators are indicated in this section.

CHAPTER 5: In this chapter we switch our attention to Toeplitz operators acting on the standard weighted Bergman spaces over a bounded symmetric domain. For a fixed measurable bounded symbol we investigate the uniform compactness (w.r.t. the standards weights) of Toeplitz operators with bounded symbols acting on these Bergman spaces. In Section 5.1 we motivate this problem by discussing some of the results in [15] and we state some of our main results. Section 5.2 is devoted to some preliminaries on bounded symmetric domains as well as the standard weighted Bergman spaces over such domains. In Section 5.3 we will construct a trace class operator (c.f. above) and we will indicate some generalized Forelli-Rudin inequalities. Together with a standard estimate for the trace norm, this enables us to obtain Theorem 7. In Section 5.4 we prove the equivalence in Theorem 8 by using the density theorem obtained in Chapter 1 and another result in [77]. Finally, we mention some open problems which are inspired by our results

Appendix: We collect several calculations which are essential throughout the thesis. As the proof of these calculations are not needed somewhere else we provide them in the Appendix.

We remark that the results in Chapters 2 and 5 originally appeared as an articles in $[21,113]$ with minor modifications.

\section{Acknowledgments}

I would like to express my sincerest gratitude to my supervisor, Dr. W. Bauer who has been most generous with his time and ideas. In all the time of the research and writing this thesis he provided encouragements and useful hints. The thesis would not have been possible without his assistance, his patience and his tolerance. 
I am indebted to Prof. Dr. I. Witt for his interest in examining this thesis. I am grateful to Dr. J. Isralowitz for valuable conversations and for correcting my English. In addition, I thank the DFG for financial support during these years.

I owe my loving to my parents Ahmad and Soheir Issa. Their emotional support, confidence and encouragement helped me a lot. Despite the geographical distance, my sisters Doaa, Fatima and Zeinab Issa were always nearby. I wish also to express my heartfelt thanks to my wife Aliye $\mathrm{K}$. Issa for her understanding and patience during the last two years. She is my shadow, my light and all my life. To you my love, I promise we would be always together and forever. Finally, it is my pleasure to dedicate this thesis to M. Ibn L. Hassan Al-Moaammal who has been nearby encouraging me during my research. 


\section{Chapter 1}

\section{Preliminaries}

\subsection{Reproducing kernel Hilbert spaces}

In this section, we present some fundamental properties of reproducing kernel Hilbert spaces and we show that the Berezin transform acting on bounded operators is one-to-one. We start by some preliminaries.

Definition 1.1.1. Let $H$ be a Hilbert space (over $\mathbb{C}$ ) of complex valued functions on a set $\Omega \subset \mathbb{C}^{n} . H$ is called a reproducing kernel Hilbert space (abbreviated by RKHS) if for every $z \in \Omega$ the linear evaluation map, $\delta_{z}: H \longrightarrow \mathbb{C}$, defined by $\delta_{z}(f)=f(z)$ is continuous.

For a Hilbert space $H$ we denote by $\langle\cdot, \cdot\rangle$ the inner product on $H$ and by $\|\cdot\|$ the corresponding norm. We remark that $H$ is a RKHS if and only if for every $z \in \Omega$ there exists a unique function $K_{z} \in H$ such that

$$
f(z)=\left\langle f, K_{z}\right\rangle, \quad \text { for all } f \in H .
$$

Indeed, the necessity follows from the Riesz Representation Theorem and the sufficiency is direct:

$$
\left|\delta_{z}(f)\right|=\left|\left\langle f, K_{z}\right\rangle\right| \leq\|f\|\left\|K_{z}\right\|
$$

Definition 1.1.2. The 2-variable function on $\Omega \times \Omega$ defined by

$$
K(w, z)=K_{z}(w)
$$

is called the reproducing kernel of $H$.

Note that, there are many Hilbert spaces of functions which are not RKHS. Indeed, when $\Omega$ is an open connected subset of $\mathbb{C}^{n}$ then the ordinary Lebesgue space $L^{2}(\Omega)$ is not a RKHS. In fact, for any $z_{0} \in \Omega$ we can construct a sequence $\left\{f_{n}\right\} \subset C_{c}^{\infty}(\Omega)$ such that $\left\|f_{n}\right\|_{L^{2}(\Omega)}=1$ and $f_{n}\left(z_{0}\right)=n$ for all $n \in \mathbb{N}$ and thus the evaluation map is not continuous. 
The general theory of reproducing kernel Hilbert spaces was systematized in [6, 7] by $\mathrm{N}$. Aronszajn around 1948 and developed by L. Schwartz [150]. However, before N. Aronszajn there have been two trends in consideration of reproducing kernels. The first trend considered positive definite kernels, which were discovered by J. Mercer [133, 134] and E. H. Moore [135], and study the kernel itself. The kernels considered by J. Mercer were „,continuous kernels of positive definite integral operators" [7]. They arose in the theory of integral equations as developed by Hilbert and they were positive definite in the sense of E. H. Moore. Later on, E. H. Moore $[135,136]$ considered such kernels on an abstract set and he discovered that to each positive definite kernel there corresponds a Hilbert space of functions to which these kernels has the reproducing kernel property (1.1.1). The second trend considered a Hilbert space of functions and the corresponding kernel is used as a tool in the study of these functions. In the investigation of S. Zaremba on boundary value problems for harmonic and biharmonic functions, he was the first to go through this trend and to find a reproducing kernel for the classes of these functions (c.f. [180]). Then the idea of reproducing kernels appeared in the work done by S. Bergman [36, 37]. He introduced reproducing kernels for the classes of harmonic and analytic functions and he called them kernel functions. In [44-46], S. Bergman and M. Schiffer used the original idea of Zaremba in applying the kernels to the solutions of boundary value problems. It turned out that these kernels are useful tools for solving boundary value problems of partial differential equations of elliptic type. Finally, N. Aronszajn [6] found that any reproducing kernel on a Hilbert space of functions is positive definite in the sense of E. H. Moore and thus formed the second link between these two trends. This link led to important results in the study of conformal mappings [38, 39, 41], integral equations [133], partial differential equations [40], invariant Riemannian metrics [42], in probability theory [121, 141, 142] and in other subjects in physics. For instant, in [70] J. Duchon formulated generalized smooth surface spline functions using the reproducing kernel Hilbert space technique in Sobolev space [1]. Together with the work of Loéve the theory of reproducing kernel Hilbert space has been used for a variety of applications, especially in data interpolation, signal processing, and smoothing (c.f. [48, 140, 173]). Meanwhile, the notion of RKHS is quite used in numerous fields especially in solving boundary value problems [89-91], Tikhonov regularization [105, 159], image and video colorization [100].

In our study there are several reasons for introducing the abstract theory of reproducing kernel Hilbert spaces. The two main tasks in the thesis the Segal-Bargmann space and the Bergman spaces over bounded symmetric domains are special types of a RKHS. The corresponding kernels of these spaces share some fundamental properties. In particular, they can be calculated explicitly by finding an orthonormal system of functions in the corresponding space (c.f. Proposition 1.1.3 below). Moreover, the fact that the Berezin transform is one-to-one on bounded Toeplitz operators acting on a Bergman space follows from the fact that this transform is one-to-one on bounded operators acting on any RKHS of analytic functions whose kernel does not vanish on the diagonal of $\Omega \times \Omega$ (c.f. Proposition 1.1.4 below).

Let us now present some basic properties of the reproducing kernel $K$ in a RKHS. Applying 
(1.1.1) to the function $K_{z}$ at $w$, we get

$$
K(w, z)=K_{z}(w)=\left\langle K_{z}, K_{w}\right\rangle \quad \text { for } z, w \in \Omega .
$$

Therefore, the reproducing kernel satisfies:

$$
K(w, z)=\overline{K(z, w)}, \quad\left\|K_{z}\right\|=\sqrt{K(z, z)}, \quad \text { and } \quad|K(w, z)| \leq \sqrt{K(z, z)} \sqrt{K(w, w)} .
$$

Furthermore, for a fixed $z_{0} \in \Omega$ it follows that $K\left(z_{0}, z_{0}\right)=0$ if and only if $f\left(z_{0}\right)=0$ for all $f \in H$. Again by the reproducing kernel property (1.1.1) it is easy to check that $\operatorname{span}\left\{K_{z}\right\}_{z \in \Omega}$ is dense in $H$.

It is important to notice that if the RKHS $H$ with kernel $K$ is a subspace of a larger Hilbert space then $K$ determines the orthogonal projection to $H$.

Proposition 1.1.1. [151] Let $(L,\langle\cdot, \cdot\rangle)$ be a Hilbert space of complex valued functions defined on $\Omega$. Equipped with the norm inhereted from $L$, suppose there is a reproducing kernel Hilbert space $H \subset L$. Then the orthogonal projection $P: L \longrightarrow H$ is given by

$$
[P f](z)=\left\langle f, K_{z}\right\rangle, \quad f \in L, z \in \Omega \text {. }
$$

Proof. Let $f \in L$, then $[P f](z)=\left\langle P f, K_{z}\right\rangle=\left\langle f, P K_{z}\right\rangle=\left\langle f, K_{z}\right\rangle$.

The next proposition shows that the reproducing kernel of a separable RKHS can be described explicitly in terms of an orthonormal basis for the space.

Proposition 1.1.2. [151] Let $H$ be a separable RKHS. Then for any complete orthonormal system $\left\{e_{n}\right\}_{n \in \mathbb{N}}$ we have

$$
K(w, z)=\sum_{n \in \mathbb{N}} e_{n}(w) \overline{e_{n}(z)},
$$

where the series converges absolutely on $\Omega \times \Omega$.

Proof. Since $\left\{e_{n}\right\}_{n \in \mathbb{N}}$ is a complete orthonormal system then for any $z \in \Omega$ we have $K_{z}=$ $\sum_{n \in \mathbb{N}}\left\langle K_{z}, e_{n}\right\rangle e_{n}$, where the series converges in $H$. Hence

$$
K_{z}(w)=\left\langle K_{z}, K_{w}\right\rangle=\sum_{n \in \mathbb{N}}\left\langle K_{z}, e_{n}\right\rangle\left\langle e_{n}, K_{w}\right\rangle=\sum_{n \in \mathbb{N}} \overline{e_{n}(z)} e_{n}(w) .
$$

The absolute convergence follows by Cauchy-Schwartz inequality and Parseval identity:

$$
\sum_{n \in \mathbb{N}}\left|\overline{e_{n}(z)} e_{n}(w)\right| \leq\left(\sum_{n \in \mathbb{N}}\left|\left\langle K_{z}, e_{n}\right\rangle\right|^{2}\right)^{\frac{1}{2}}\left(\sum_{n \in \mathbb{N}}\left|\left\langle e_{n}, K_{w}\right\rangle\right|^{2}\right)^{\frac{1}{2}}=\left\|K_{z}\right\|\left\|K_{w}\right\| .
$$

Hereafter, we confine our discussion to the case where $\Omega$ is an open connected domain in $\mathbb{C}^{n}$ 
Proposition 1.1.3. Let $H$ be a RKHS of analytic functions on $\Omega$. Then the kernel $K(w, z)$ is analytic in $w$ and anti-analytic in $z$. Moreover, $H$ is separable and the convergence in (1.1.3) is uniform on compact sets of $\Omega \times \Omega$.

Proof. The first part of the proposition follows since $K_{z} \in H$ is analytic and $K(w, z)=$ $\overline{K(z, w)}$. Note that, for any dense sequence $\left\{z_{m}\right\}$ in $\Omega$ the corresponding sequence of functions $\left\{K_{z_{m}}\right\}$ is a total set in $H$ therefore $H$ is separable. Moreover, if $S \subset \Omega$ is compact we know that for any $f \in H$ the complex valued function $z \longmapsto f(z)=\left\langle f, K_{z}\right\rangle$ is analytic hence continuous on $\Omega$. Therefore, the Hilbert space-valued function $z \longmapsto K_{z}$ is weakly continuous. So that, $\left\{K_{z}, z \in S\right\}$ is weakly compact hence strongly bounded. This means that $\sup \left\{\left\|K_{z}\right\|, z \in S\right\}$ is finite and the uniform convergence follows from (1.1.4).

The bounded operators on a RKHS admit an interesting representation via the so-called Berezin transform. This transform plays an important role in the theory of Bergman spaces as well as in the solution of the heat equation (c.f. Section 3). Let us start by recalling the definition of this transform.

Definition 1.1.3. Let $H$ be a RKHS such that $K(z, z)>0$ for all $z \in \Omega$. Let $T$ be a linear operator acting on the linear space

$$
\mathcal{L}_{H}:=\operatorname{span}\left\{k_{z}:=\frac{1}{\sqrt{K(z, z)}} K_{z}\right\}_{z \in \Omega},
$$

of the normalized reproducing kernel functions with values in $H$. Then the Berezin transform of $T$, denoted by $\widetilde{T}$, is the complex valued function defined on $\Omega$ by:

$$
\widetilde{T}(z)=\left\langle T k_{z}, k_{z}\right\rangle
$$

The fact that the Berezin transform acting on the bounded operators of a RKHS forms a representation of these operators is a special case of a more general statement given in the next proposition. It is sufficient to find a Hilbert subspace $H_{0}$ in $H$ such that $\mathcal{L}_{H} \subset H_{0}$ is dense then for any $T \in \mathcal{L}\left(H_{0}, H\right)$ one has $\widetilde{T}=0$ if and only if $T=0$. This idea was used in [14] for the case of the Segal-Bargmann space to prove that the Berezin transform acting on Toeplitz operators with symbols having a certain growth at infinity is one-to-one. In the next chapter, we use a similar argument to that in [14] to prove that two Toeplitz operators with reasonable symbol growth at infinity commute on the space of holomorphic polynomials if and only if the Berezin transform of their commutator vanishes (c.f. Corollary 2.2.1).

Proposition 1.1.4. Let $H$ be a RKHS of analytic functions on $\Omega$. Suppose there is a Hilbert space $H_{0}$ of analytic functions on $\Omega$ such that $\mathcal{L}_{H} \subset H_{0}$ is dense in $H_{0}$. Then the mapping $T \longrightarrow \widetilde{T}$ is an injective linear operator from $\mathcal{L}\left(H_{0}, H\right)$ into the real-analytic functions in $L^{\infty}(\Omega)$.

Proof. Using the continuity of $T$ we have

$$
|\widetilde{T}(z)|=\left|\left\langle T k_{z}, k_{z}\right\rangle\right| \leq\left\|T k_{z}\right\| \leq\|T\|,
$$


hence $\widetilde{T} \in L^{\infty}(\Omega)$. Moreover, since $K(w, z)$ is analytic in $w$ conjugate-analytic in $z$ then $k_{z}$ is real-analytic but $T$ is continuous hence $\widetilde{T}(z)$ is real-analytic too. To prove the injectivity, we define the two-variable function on $\Omega \times \Omega$

$$
K_{T}(z, w):=\left\langle T K_{w}, K_{z}\right\rangle=\left(T K_{w}\right)(z)
$$

Since $T K_{w} \in H$ for all $w \in \Omega$ the kernel $K_{T}$ is analytic in $z$ but

$$
K_{T}(z, w)=\left\langle K_{w}, T^{\star} K_{z}\right\rangle=\overline{\left\langle T^{\star} K_{z}, K_{w}\right\rangle}=\overline{\left(T^{\star} K_{z}\right)(w)},
$$

hence $K_{T}$ is anti-analytic in $w$. By assumption $K_{T}$ vanishes on the diagonal of $\Omega \times \Omega$. As a consequence (c.f. p. 371 in [124]), the map $K_{T}$ is identically zero on $\Omega \times \Omega$ hence $T K_{w} \equiv 0$ for all $w \in \Omega$. Since $\mathcal{L}_{H} \subset H_{0}$ is dense and $T$ is continuous on $H_{0}$, it follows that $T=0$.

At the end of this section we give some classical examples of reproducing kernel Hilbert spaces.

Example 1.1.1. It is well known that the Peter-Wiener space $P W_{r}$ which consists of square integrable functions on $\mathbb{R}$ such that their Fourier transformation vanishes outside the band $[-r, r]$ is a RKHS (c.f. [179]). We generalize this fact to arbitrary dimensions and calculate the reproducing kernel of the corresponding space. For each $r>0$, we define the generalized Peter-Wiener space $P W_{r}$ on $\mathbb{R}^{n}$ to be (c.f. [178])

$$
\begin{aligned}
& P W_{r}\left(\mathbb{R}^{n}\right):=\left\{\varphi: \mathbb{C}^{n} \longrightarrow \mathbb{C} \mid \varphi \text { is entire, } \varphi_{\mid \mathbb{R}^{n}} \in L^{2}\left(\mathbb{R}^{n}\right), \text { s. t. for any } \epsilon>0\right. \\
&\left.\exists C_{\epsilon} \text { with }|\varphi(z)| \leq C_{\epsilon} e^{(1+\epsilon)|z|}, \forall z \in \mathbb{C}^{n}\right\} .
\end{aligned}
$$

According to the generalized Paley-Wiener theorem (c.f. [156, 178]) we know that

$$
\mathcal{F}\left(L^{2}(B(0, r))\right)=P W_{r}\left(\mathbb{R}^{n}\right),
$$

where $B(0, r)$ is the closed ball in $\mathbb{R}^{n}$ with radius $r$ centered at the origin, and $\mathcal{F}$ is the $n$ dimensional Fourier transformation. Since $\mathcal{F}$ is an isometry, we can endow the space $P W_{r}$ with the following inner product

$$
\langle f, g\rangle_{P W_{r}}=\left\langle\mathcal{F}^{-1} f, \mathcal{F}^{-1} g\right\rangle_{L^{2}(B(0, r))}
$$

It follows directly that $P W_{r}$ is a Hilbert space and for every $z \in \mathbb{C}^{n}$

$$
\begin{aligned}
|\varphi(z)| & =(2 \pi)^{-n} \int_{B(0, r)} e^{-i z \xi}\left(\mathcal{F}^{-1} \varphi\right)(\xi) d \xi \\
& \leq(2 \pi)^{-n} \sqrt{|B(0, r)|} e^{r|z|}\left\|\mathcal{F}^{-1} \varphi\right\|_{L^{2}(B(0, r))}=C\|\varphi\|_{P W_{r}},
\end{aligned}
$$

which shows that $P W_{r}$ is a RKHS. Moreover, for any $\varphi \in P W_{r}$ and any $z \in \mathbb{C}^{n}$ the identity

$$
\varphi(z)=\left\langle\mathcal{F}^{-1} \varphi, e^{i z(\cdot)}\right\rangle_{L^{2}(B(0, r))}=\left\langle\varphi, \mathcal{F} e^{i z(\cdot)}\right\rangle_{P W_{r}},
$$


shows that the reproducing kernel of $P W_{r}$ is $K(w, z)=\mathcal{F}\left(e^{i z(\cdot)}\right)(w)$. Using induction on $n$, one can prove that (c.f. [65])

$$
\mathcal{F}\left(e^{i z(\cdot)}\right)(w)=\left(\frac{r}{2 \pi}\right)^{\frac{n}{2}} \frac{J_{\frac{n}{2}}(r|z-w|)}{|z-w|^{\frac{n}{2}}},
$$

where $J_{v}$ is the Bessel function of the first kind of order $v$.

Other examples of RKHS are the Bergman spaces, the Sobolev spaces $H^{s}\left(\mathbb{R}^{d}\right)$ for all $s>\frac{d}{2}$ [47], the Szegö spaces $A l_{2}(\partial \Omega)$ and many others [151]. In the next section we discuss briefly the Bergman spaces over an open domain.

\subsection{Bergman spaces}

In the book [43], S. Bergman developed an elegant theory of the Hilbert spaces of analytic square-integrable (with respect to the volume measure) functions over a bounded domain $\Omega \subset$ $\mathbb{C}^{n}$. These spaces turned out to be reproducing kernel Hilbert spaces. They are now called Bergman spaces and the corresponding kernels are the so-called Bergman kernels. However, for an arbitrary (not necessarily bounded) open connected domain $\Omega$ one can choose an appropriate weight function $w$ on $\Omega$ and then consider the analytic functions which are square integrable with respect to the weight function. It was natural to call these spaces ,weighted Bergman spaces". We start this section, by introducing these spaces and proving that they possess a reproducing kernel.

We will identify $\Omega$ (not necessarily bounded) as subset of $\mathbb{R}^{2 n}$ and we write $z_{k}=x_{k}+i y_{k}$ for any $z=\left(z_{1}, \cdots, z_{n}\right) \in \Omega \subseteq \mathbb{C}^{n}$. We also write $z=r \xi$ for the representation of $z$ in polar coordinates, i.e. $r \in[0, \infty)$ and $\xi$ is in the real $(2 n-1)$-dimensional unit sphere $\mathbb{S}^{2 n-1} \subset \mathbb{C}^{n}$. We denote by $d v(z)$ the ordinary Lebesgue measure on $\Omega$

$$
d v(z)=\prod_{k=1}^{n} d x_{k} d y_{k}
$$

Let $w: \Omega \longrightarrow \mathbb{R}$ be a positive integrable function that is locally bounded below by a strictly positive constant, i.e. for every $z \in \Omega$ there exists a neighborhood $N_{z} \subset \Omega$ and a contant $M_{z}$ such that $w(u) \geq M_{z}>0$ for all $u \in N_{z}$. We denote by $L_{w}^{2}:=L^{2}\left(\Omega, d \mu_{w}\right)$ the Hilbert space of functions on $\Omega$ which are square integrable with respect to the finite measure

$$
d \mu_{w}(z):=w(z) d v(z)
$$

Definition 1.2.1. The weighted Bergman space over $\Omega$ (with respect to the weight $w$ ), denoted by $H_{w}^{2}(\Omega)$, is the space of all holomorphic functions $f$ on $\Omega$ such that

$$
\|f\|_{H_{w}^{2}(\Omega)}^{2}:=\int_{\Omega}|f(z)|^{2} w(z) d v(z)<\infty .
$$


Note that when $\Omega$ is bounded and $w \equiv \operatorname{vol}(\Omega)^{-1}$ then $H_{w}^{2}(\Omega)$ is the standard ,unweighted" Bergman space. The following lemma is a generalization of Lemma 1.4.1 in [124] from the „unweighted" to the weighted case of Bergman spaces. It shows that the convergence in the norm of $H_{w}^{2}(\Omega)$ implies the uniform convergence on compact sets.

Lemma 1.2.1. [124] Let $K \subset \Omega$ be a compact set. Then there exists a constant $C_{K}$ depending on $K$ and $w$, such that

$$
\sup _{z \in K}|f(z)| \leq C_{K}\|f\|_{H_{w}^{2}(\Omega)}, \quad \text { for all } f \in H_{w}^{2}(\Omega)
$$

In particular, the pointwise evaluation map is continuous on $H_{w}^{2}(\Omega)$.

Proof. Since $K$ is compact and $w$ is locally bounded below, there exist $r, M>0$ depending on $K$ and $w$ such that the closed ball $\overline{B(z, r)} \subset \Omega$ for any $z \in K$ and $w(u) \geq M$ for all $u \in B(z, r)$. By the mean-value property of harmonic functions (c.f. p. 313 in [157] for example), we know that for any $f \in H_{w}^{2}(\Omega)$ and any $0<\rho \leq r$ we have:

$$
f(z)=\frac{1}{\sigma\left(\mathbb{S}^{2 n-1}\right)} \int_{\mathbb{S}^{2 n-1}} f(z+\rho \xi) d \sigma(\xi)
$$

where $d \sigma$ is the area measure on $\mathbb{S}^{2 n-1}$. Multiplying both sides of (1.2.2) by a factor of $\rho^{2 n-1}$ and integrating, we obtain

$$
\begin{aligned}
f(z) \frac{r^{2 n}}{2 n} & =\frac{1}{\sigma\left(\mathbb{S}^{2 n-1}\right)} \int_{0}^{r} \int_{\mathbb{S}^{2 n-1}} f(z+\rho \xi) \rho^{2 n-1} d \rho d \sigma(\xi) \\
& =\frac{1}{\sigma\left(\mathbb{S}^{2 n-1}\right)} \int_{B(z, r)} f(u) d v(u)
\end{aligned}
$$

Hence by Cauchy-Schwartz inequality we have

$$
\begin{aligned}
|f(z)| & \leq \frac{1}{|B(z, r)|}\left[\int_{B(z, r)}|f(u)|^{2} d v(u)\right]^{\frac{1}{2}}\left[\int_{B(z, r)} d v(u)\right]^{\frac{1}{2}} \\
& =\frac{1}{\sqrt{|B(z, r)|}}\left[\int_{B(z, r)}|f(u)|^{2} d v(u)\right]^{\frac{1}{2}} \\
& \leq \frac{M^{-\frac{1}{2}}}{\sqrt{|B(z, r)|}}\left[\int_{B(z, r)}|f(u)|^{2} w(u) d v(u)\right]^{\frac{1}{2}} \\
& =C_{K}\|f\|_{H_{w}^{2}(\Omega)} .
\end{aligned}
$$

Remark 1.2.1. The proof of the above lemma shows that the inequality (1.2.1) is still true for any harmonic function $f$ in $L_{w}^{2}$.

We use Lemma 1.2.1 to prove that $H_{w}^{2}(\Omega)$ is a RKHS. 
Theorem 1.2.1. [124] Let $\Omega$ be an open domain in $\mathbb{C}^{n}$, and $w$ be a positive locally bounded below measurable function. Then the Bergman space $H_{w}^{2}(\Omega)$ is a reproducing kernel Hilbert space. Moreover, for any orthonormal basis $\left\{e_{n}\right\}_{n \in \mathbb{N}}$ of $H_{w}^{2}(\Omega)$ the reproducing kernel is given by

$$
K(w, z)=\sum_{n \in \mathbb{N}} e_{n}(w) \overline{e_{n}(z)}
$$

where the series converges uniformly on compact sets of $\Omega \times \Omega$.

Proof. For $H_{w}^{2}(\Omega)$ to be a RKHS we only need to prove the completeness. Let $\left\{f_{n}\right\}$ be a Cauchy sequence in $H_{w}^{2}(\Omega)$. By the previous lemma, $f_{n}$ convergence uniformly to some function $g(z)$ on every compact set of $\Omega$. Hence, by Montel's Theorem, $g$ is holomorphic on $\Omega$. Since $L_{w}^{2}$ is complete, there exists $f \in L^{2}\left(\Omega, d \mu_{w}\right)$ such that $f_{n}$ converges to $f$ in the norm. Therefore, there is a subsequence $f_{n_{k}}$ which converges to $f$ pointwisely a.e. on $\Omega$. This shows that $f=$ $g \in H_{w}^{2}(\Omega)$ hence $H_{w}^{2}(\Omega)$ is complete. Since $L^{2}\left(\Omega, d \mu_{w}\right)$ is separable, the second part of the theorem follows directly from Proposition 1.1.3.

Note that, if $w$ is an integrable positive function then $f(z) \equiv 1 \in H_{w}^{2}(\Omega)$ hence $K(z, z)>0$ for all $z \in \Omega$. Furthermore, by Proposition (1.1.1) the orthogonal projection $P$ from $L_{w}^{2}$ onto $H_{w}^{2}(\Omega)$ is an integral operator given by:

$$
[P h](z)=\langle h, K(\cdot, z)\rangle=\int_{\Omega} h(u) K(z, u) d \mu_{w}(u), \quad \forall z \in \Omega
$$

Now, we introduce the so-called Toeplitz operators on the weighted Bergman spaces. We note that the notion of Toeplitz operators goes back to the work of Otto Toeplitz [163] on the Hardy space $H^{2}\left(\mathbb{S}^{1}\right)$. This space consists of all square integrable functions on the unit circle whose discrete Fourier transform vanishes for all negative integers. As a consequence, these functions admit a holomorphic extension to the unit disc. Moreover, it turned out that $H^{2}\left(\mathbb{S}^{1}\right)$ is a RKHS with the topology induced from the norm of $L^{2}\left(\mathbb{S}^{1}, \frac{1}{2 \pi} d \theta\right)(d \theta$ denotes the ordinary measure on $\mathbb{S}^{1}$ ). For a bounded function $a \in L^{\infty}\left(\mathbb{S}^{1}\right)$, O. Toeplitz defined the operator $T_{a}$ : $H^{2}\left(\mathbb{S}^{1}\right) \longrightarrow H^{2}\left(\mathbb{S}^{1}\right)$, by the rule $T_{a}(f)=P_{h}(a f)$ where $P_{h}$ is the orthogonal projection form $L^{2}\left(\mathbb{S}^{1}, \frac{1}{2 \pi} d \theta\right)$ onto $H^{2}\left(\mathbb{S}^{1}\right)$. These operators have several remarkable properties. For example, a necessary and a sufficient condition that an operator on $H^{2}\left(\mathbb{S}^{1}\right)$ is a Toeplitz operator is that its matrix (with respect to the monomials) is a Toeplitz matrix [54]. It is also well known that there are no non-zero compact Toeplitz operators on the Hardy space (c.f. Ch. 10 in [186]) and that the spectrum of these operators is always connected (see [69]). However, it was important to extend the theory of Toeplitz operators to the case of analytic square integrable functions on an open domain. That is replacing the Hardy space by the Bergman space so it was natural to call these operators Toeplitz operators on the Bergman space. The study of these operators is ,a core part of the so-called „quantization” of symplectic manifolds” [166] . In fact, the use of Toeplitz operators in geometric quantization has its origin in the work of F. Berezin [29-32] and L. Boutet de Monvel [50-52]. The idea of quantization is that of assigning to functions on a symplectic manifold operators on a separable Hilbert space (state space) satisfying certain 
commutation relations (c.f. [99]). The quantization also consists in deforming the pointwise product of functions into a new product depending on a positive parameter (Planck's constant) $[49,62,76,99]$. It was noticed that there are many differences between the theory of Toeplitz operators on the Bergman space and that on the Hardy space. For example, it is easy to construct a Toeplitz operator on the Bergman space whose spectrum is discrete (see [78] for example). In the following we will prove that the space of Toeplitz operators with continuous symbols of compact support is norm dense in the space of all compact operators on the weighted Bergman space. This fact, appeared for the first time in the work of M. Engliš [74] for the case of the Bergman space over the unit disc and in the work of L. Coburn and C. Berger [35] for the case of the Segal-Bargmann space. We will show that this property is still true for any weighted Bergman space over any open domain. We start by defining the Toeplitz operators and recalling some basic properties.

Definition 1.2.2. For a measurable symbol $g: \Omega \longrightarrow \mathbb{C}$ we define the operator $T_{g}$ on:

$$
D\left(T_{g}\right):=\left\{h \in H_{w}^{2}(\Omega) \mid g h \in L^{2}\left(\Omega, d \mu_{w}\right)\right\}
$$

by

$$
T_{g} h=P(g h) \in H_{w}^{2}, \quad h \in D\left(T_{g}\right) \subset H_{w}^{2}(\Omega) .
$$

$T_{g}$ is called the Toeplitz operator on the weighted Bergman space $H_{w}^{2}(\Omega)$ with symbol $g$.

Since $\left(\Omega, \mu_{w}\right)$ is a finite measure space, for each measurable function $g \in L^{\infty}(\Omega)$ the Toeplitz operator $T_{g}$ is defined on $H_{w}^{2}(\Omega)$. Moreover, $T_{g} \in \mathcal{L}\left(H_{w}^{2}(\Omega)\right)$ with $\left\|T_{g}\right\| \leq\|g\|_{\infty}$. If we suppose further that $g$ has a compact support in $\Omega$ then $T_{g}$ is of trace class on $H_{w}^{2}(\Omega)$. To make this clear, recall the well known result for operators on any measurable space (Theorem 3.5 in [186]):

Lemma 1.2.2. [186] Let $(X, \mu)$ be a measure space, and $T$ a linear operator on $L^{2}(X, d \mu)$. Then $T$ is a Hilbert-Schmidt operator if and only if there is a function $K \in L^{2}(X \times X, d \mu \times d \mu)$ such that:

$$
T f(x)=\int_{X} K(x, y) f(y) d \mu(y), \quad \text { for all } f \in L^{2}(X, d \mu) .
$$

Now suppose $g$ is a bounded measurable function with a compact support $S_{g} \subset \Omega$. Let $\chi$ be the characteristic function of $S_{g}$. We will prove that $T_{g}$ is of trace class on $H_{w}^{2}(\Omega)$. On the one hand, the operator $P M_{\chi}$, where $M_{\chi}$ is the multiplication operator by $\chi$, is a Hilbert-Schmidt operator on $L^{2}\left(\Omega, d \mu_{w}\right)$. Indeed, by (1.2.4) we know that for any $h \in L^{2}\left(\Omega, d \mu_{w}\right)$ and any $z \in \Omega$

$$
P M_{\chi} h(z)=\langle\chi h, K(\cdot, z)\rangle=\int_{\Omega} \chi(u) h(u) K(z, u) d \mu_{w}(u) .
$$

Applying the above lemma to $T=P M_{\chi}, X=\Omega$, and $d \mu=d \mu_{w}$ we need to show that $\chi(u) K(z, u) \in L^{2}\left(\Omega \times \Omega, d \mu_{w} \times d \mu_{w}\right)$. But this is clear since the mapping $\Omega \ni u \longrightarrow K(u, u)$ is continuous and

$$
\int_{\Omega}|\chi(u)|^{2} \int_{\Omega} K(z, u) \overline{K(z, u)} d \mu_{w}(z) d \mu_{w}(u)=\int_{S_{g}} K(u, u) d \mu_{w}(u) .
$$


On the other hand, since $g$ is bounded the multiplication operator $M_{g}$ is also bounded on $L^{2}\left(\Omega, d \mu_{w}\right)$. Hence $\left(P M_{\chi}\right) M_{g}\left(P M_{\chi}\right)^{*}$ is of trace class on $L^{2}\left(\Omega, d \mu_{w}\right)$ (c.f. Lemma 1.36 in [186]). But the Toeplitz operator $T_{g}$ coincides with $\left(P M_{\chi}\right) M_{g}\left(P M_{\chi}\right)^{*}$ on $H_{w}^{2}(\Omega)$. Indeed, for $f, h \in H_{w}^{2}(\Omega)$ we have

$$
\left\langle T_{g} f, h\right\rangle=\left\langle P M_{g} f, h\right\rangle=\left\langle P M_{g} M_{\chi}^{*} P f, h\right\rangle=\left\langle P M_{\chi} M_{g} M_{\chi}^{*} P f, h\right\rangle=\left\langle P M_{\chi} M_{g}\left(P M_{\chi}\right)^{*} f, h\right\rangle .
$$

Similar to a corresponding result in [35] on Toeplitz operators acting on the Segal-Bargmann space, and by purely functional analytic methods one can prove the following density criteria for Bergman spaces over any open domain (c.f. Appendix A.1 for a complete proof):

Theorem 1.2.2. For any open domain $\Omega \subseteq \mathbb{C}^{n}$ the space

$$
C \equiv\left\{T_{g} \quad \mid \quad g \text { continuous with compact support in } \Omega\right\}
$$

is norm dense in the space of all compact operators acting on $H_{w}^{2}(\Omega)$.

Note that, Theorem 17 in [35] shows that the bounded Toeplitz operators are in general not norm-dense in the algebra of all bounded operators on $H_{w}^{2}(\Omega)$.

In the next two sections, we consider two examples of Bergman spaces the Segal-Bargmann space ( $\Omega=\mathbb{C}^{n}$ with natural Gaussian measures) and the standard weighted Bergman spaces over the unit ball of $\mathbb{C}^{n}$.

\subsection{Segal-Bargmann space}

The Segal-Bargmann space on $\mathbb{C}^{n}$,dates back to the work of Fischer in mathematics [82] and Fock in physics [83]" [155]. For this reason it is sometimes called the Fischer or the Fock space. In quantum mechanics it is a sort of ,phase space" that incorporates the states of a dynamical system. [99]. Recall that in classical mechanics the motion of a system of $n$ particles is described by their corresponding position $\left(x_{1}, \cdots, x_{n}\right)$ and momentum $\left(p_{1} \cdots, p_{n}\right)$. The energy of the system is given by the Hamiltonian function $H(x, p)$ defined on the phase space $\mathbb{R}^{2 n}$. It is well known that if $f$ is a smooth function on $\mathbb{R}^{2 n}$ and $(x(t), p(t))$ is a solution of Hamilton's equations then $\frac{d f}{d t}=\{f, H\}$ on $(x(t), p(t))$ where $\{\cdot, \cdot\}$ is the Poisson bracket (c.f. [99]). In quantum mechanics, the phase space is replaced by an infinite-dimensional separable complex Hilbert space $\mathcal{H}$ and points $(x, p) \in \mathbb{R}^{2 n}$ are replaced by unit vectors in $\mathcal{H}$ called the states. The real valued functions which were defined in the phase space in classical mechanics are now replaced by self-adjoint operators on $\mathcal{H}$. In particular, we have the position and momentum operators $P_{j}, Q_{j}$ where $j=1, \cdots, n$ (c.f. p. 83 in [160] for explicit definition of these operators). According to Dirac the relations between these operators are given by the canonical commutation relations (CCR) for $n$ degrees of freedom which is a classical analogue to the Poisson bracket in classical mechanics (p. 82 in [160])

$$
\left[P_{j}, P_{k}\right]=\left[Q_{j}, Q_{k}\right]=0 \quad \text { and }\left[Q_{j}, P_{k}\right]=i \hbar \delta_{j, k}
$$


where $1 \leq j, k \leq n,[\cdot, \cdot]$ denotes the commutator and $\hbar$ is the Planck's constant. The annihilation and creation operators $\left\{\mu_{j}, \bar{\mu}_{j}\right\}_{j=1 \cdots n}$ (together are called the ladder operators) are defined by

$$
\mu_{j}=\frac{1}{\sqrt{2}}\left(P_{j}+i Q_{j}\right) \quad \text { and } \quad \bar{\mu}_{j}=\frac{1}{\sqrt{2}}\left(P_{j}-i Q_{j}\right) .
$$

Using the CCR (1.3.1) one can easily check that the ladder operators satisfy

$$
\left[\bar{\mu}_{j}, \bar{\mu}_{k}\right]=\left[\mu_{j}, \mu_{k}\right]=0 \quad \text { and } \quad\left[\bar{\mu}_{j}, \mu_{k}\right]=\hbar \delta_{j, k}
$$

In 1928 Fock introduced the operator solution $\bar{\mu}_{j}=\hbar \frac{\partial}{\partial \mu_{j}}$ of the commutation relations (1.3.2) and applied it to quantum field theory [83]. In [12], V. Bargmann studied the Hilbert space on which Fock's solution is realized i.e. the Hilbert space of holomorphic functions, say in $z=\left(z_{1}, \cdots, z_{n}\right)$, so that if $\mu_{j}$ is the multiplication operator by $z_{j}$ then $\hbar \frac{\partial}{\partial z_{j}}$ is the adjoint operator. It is often named afterafter the investigation of Bargmann [12, 13] and Segal [152, 153]. In this section, we introduce the Segal-Bargmann space as well as the Toeplitz operators acting on it. We also study the Berezin transform of suitable functions and the connection of its analytic properties to the behavior of the corresponding Toeplitz operator on the SegalBargmann space.

It will be convenient to use the standard notation and to write $z \xi=\sum_{k=1}^{n} z_{k} \xi_{k},|z|=\sqrt{z \bar{z}}$, $z^{\alpha}=z_{1}^{\alpha_{1}} \cdots z_{n}^{\alpha_{n}}$ where $z, \xi \in \mathbb{C}^{n}, \alpha=\left(\alpha_{1}, \alpha_{2}, \cdots, \alpha_{n}\right) \in \mathbb{N}_{0}^{n}$ is a multi-index of non-negative integers. We shall also write $|\alpha|=\alpha_{1}+\alpha_{2}+\cdots+\alpha_{n}$ and $\alpha !=\alpha_{1} ! \alpha_{2} ! \cdots \alpha_{n} !$.

For each $t>0$, consider the Gauss-Weierstrass function $w_{t}$ on $\mathbb{C}^{n}$ defined by:

$$
w_{t}(z)=w_{t}(|z|)=\left(\frac{1}{4 \pi t}\right)^{n} e^{\frac{-|z|^{2}}{4 t}} .
$$

The function $w_{t}$ is also called the heat kernel since it satisfies the heat equation (Fourier law of heat conductivity)

$$
\frac{\partial w_{t}}{\partial t}=\Delta w_{t}
$$

where $\Delta$ is the the Laplace operator in $\mathbb{R}^{2 n} \approx \mathbb{C}^{n}$ and $t$ is the time. We introduce the oneparameter family of normalized Gaussian measures on $\mathbb{C}^{n}$

$$
d \mu_{t}(z)=w_{t}(z) d v(z)
$$

Denote by $L^{2}\left(\mathbb{C}^{n}, d \mu_{t}\right)$ the space of all $\mu_{t}$-square integrable functions on $\mathbb{C}^{n}$. For $f, g \in$ $L^{2}\left(\mathbb{C}^{n}, d \mu_{t}\right)$, we write $\langle f, g\rangle_{t}=\int_{\mathbb{C}^{n}} f(z) \overline{g(z)} d \mu_{t}(z)$ for the inner product and $\|f\|_{t}=\sqrt{\langle f, f\rangle_{t}}$ for the corresponding norm.

For each $t>0$, the subspace of all entire functions in $L^{2}\left(\mathbb{C}^{n}, d \mu_{t}\right)$, denoted by $H_{t}^{2}$, is called the Segal-Bargmann space. By Theorem 1.2.1 we know that $H_{t}^{2}$ is a reproducing kernel Hilbert space. We should note that for any $s, t>0$ the operator

$$
\left[U_{s, t} f\right](z):=f\left(z \sqrt{s t^{-1}}\right)
$$


unitary maps $L^{2}\left(\mathbb{C}^{n}, d \mu_{s}\right)$ onto $L^{2}\left(\mathbb{C}^{n}, d \mu_{t}\right)$ and $H_{s}^{2}$ onto $H_{t}^{2}$.

Returning back to the Segal-Bargmann space $H_{t}^{2}$, we want to calculate its reproducing kernel which we denote it by $K_{t}(w, z)$. For this, we recall that for any positive integer $n$ and for any $\alpha, \beta \in \mathbb{N}_{0}^{n}$ (c.f. Prop. 1.4 .9 in [149]):

$$
\int_{\mathbb{S}^{2 n-1}} \xi^{\alpha} \bar{\xi}^{\beta} d \sigma(\xi)= \begin{cases}\frac{2 \pi^{n} \alpha !}{(n-1+|\alpha|) !} & \alpha=\beta ; \\ 0 & \alpha \neq \beta .\end{cases}
$$

Since $w_{t}$ is radial, integration by polar coordinates shows that $\left\langle z^{\alpha}, z^{\beta}\right\rangle_{t}=0$ whenever $\alpha \neq$ $\beta$. Moreover, using (1.3.5) and the integral formula of the Gamma function one can easily check that $\left\|z^{\alpha}\right\|_{t}=2^{|\alpha|} \sqrt{\alpha ! t^{|\alpha|}}$. Each element $f \in H_{t}^{2}$ has a power series expansion $f(z)=$ $\sum_{\alpha \in \mathbb{N}_{0}} a_{\alpha} z^{\alpha}, a_{\alpha} \in \mathbb{C}$, with $\|f\|_{t}^{2}=\sum_{\alpha \in \mathbb{N}_{0}}\left|a_{\alpha}\right|^{2}\left\|z^{\alpha}\right\|_{t}^{2}$. In particular, the polynomials are dense in $H_{t}^{2}$ and

$$
\left\{e_{\alpha}^{t}(z):=\frac{z^{\alpha}}{2^{|\alpha|} \sqrt{\alpha ! t^{|\alpha|}}}\right\}_{\alpha \in \mathbb{N}_{0}}
$$

forms an orthonormal basis of $H_{t}^{2}$. Therefore, by (1.2.3) the reproducing kernel $K^{t}(w, z)$ of $H_{t}^{2}$ is given by:

$$
K^{t}(w, z)=\sum_{\alpha \in \mathbb{N}_{0}} \frac{1}{\alpha !} \frac{w^{\alpha} \bar{z}^{\alpha}}{(4 t)^{|\alpha|}}=e^{\frac{1}{4 t} w \bar{z}},
$$

and according to (1.2.4) the orthogonal projection $P_{t}$ from $L^{2}\left(\mathbb{C}^{n}, d \mu_{t}\right)$ onto $H_{t}^{2}$ can be written as an integral operator of the form:

$$
\left[P_{t} h\right](z)=\left\langle h, K_{t}(\cdot, z)\right\rangle_{t}=\int_{\mathbb{C}^{n}} h(w) e^{\frac{1}{4 t} z \bar{w}} d \mu_{t}(w), \quad \forall h \in L^{2}\left(\mathbb{C}^{n}, d \mu_{t}\right), z \in \mathbb{C}^{n}
$$

Note that, by choosing the weight function $w_{t}$ as defined in (1.3.3) we obtain a relation between the kernel on the diagonal and this weight $w_{t}(z)=\left(\frac{1}{4 \pi t}\right)^{n}\left(K_{t}(z, z)\right)^{-1}$ which is in general not true for a RKHS. Moreover, using the reproducing kernel property of the Segal-Bargmann space (1.3.8) one can easily check that for any $s, t>0$ the convolution product satisfies the semigroup identity

$$
w_{t} \star w_{s}=w_{t+s}
$$

(Throughout the thesis the convolution product is given by $f \star g(z)=\int_{\mathbb{C}^{n}} f(w) g(z-w) d v(w)$ ). Historically, the reason for choosing the Gauss-Weierstrass function as a weight is that it satisfies the following condition on the inner product imposed by Fock (c.f. [12] and the discussion at the beginning of this section):

Lemma 1.3.1. The Gauss-Weierstrass function $w_{t}$ is the unique continuous radial weight such that $\|1\|_{t}=1$ and for each $j=1, \cdots, n$ the operator of multiplication by $z_{j}$ and the differential operator $\frac{1}{4 t} \partial_{z_{j}}$ are adjoint on the Bergman space of $\mathbb{C}^{n}$ (here $\frac{1}{4 t}$ plays the role of Planck's constant). 
For convenience and throughout the thesis we write $\mathcal{H}_{t}^{2}$ for the Segal-Bargmann space $H_{\frac{1}{4 t}}^{2}$ and we use the following notation

$$
d \mu_{(t)}(z):=d \mu_{\frac{1}{4 t}}(z), K^{(t)}(w, z):=K^{\frac{1}{4 t}}(w, z),\langle\cdot, \cdot\rangle_{(t)}:=\langle\cdot, \cdot\rangle_{\frac{1}{4 t}} .
$$

Let us describe next the Toeplitz operators acting on the Segal-Bargmann space. Fix $t>0$, then for a measurable function $g: \mathbb{C}^{n} \longrightarrow \mathbb{C}$ the Toeplitz operator with symbol $g$, denoted by $T_{g}^{t}$, is defined on the maximal domain $D\left(T_{g}^{t}\right)=\left\{f \in H_{t}^{2} \mid g f \in L^{2}\left(\mathbb{C}^{n}, d \mu_{t}\right)\right\} \subset H_{t}^{2}$ and given by:

$$
T_{g}^{t} f(z)=\left[P_{t} g f\right](z)=\int_{\mathbb{C}^{n}} g(w) f(w) e^{\frac{1}{4 t} z \bar{w}} d \mu_{t}(w), \quad \forall z \in \mathbb{C}^{n}
$$

Throughout the thesis we write $\mathcal{T}_{g}^{t}:=T_{g}^{\frac{1}{4 t}}$ for the Toeplitz operator with symbol $g$ on the SegalBargmann space $\mathcal{H}_{t}^{2}=H_{\frac{1}{4 t}}^{2}$. Moreover, in the case $t=\frac{1}{4}$ we simply denote by $T_{g}$ the Toeplitz operator $T_{g}^{1}$.

Toeplitz operators on the Segal-Bargmann space have been studied by several authors [15$17,34,35,94,95]$ and the map $g \longrightarrow T_{g}^{t}$ has been considered by Berezin [29, 31, 32] and others $[33,62,98,106]$ as a natural ,quantization”. In particular, one can easily check that the operators $T_{z_{j}}^{t}$ and $T_{z_{k}}^{t}$ are densely defined on $H_{t}^{2}$. They satisfy the canonical commutation relations (1.3.2) ( the factor $\frac{1}{4 t}$ will play the role of the Planck's constant ). Now let us introduce a class of symbols $g$ where the correspondence $g \longrightarrow T_{g}^{t}$ is one-to-one.

Definition 1.3.1. For each $t>0$, we define the class of symbols $\mathcal{E}_{t}$ to be

$$
\mathcal{E}_{t}:=\left\{g: \mathbb{C}^{n} \longrightarrow \mathbb{C} \mid g \text { is measurable and } \exists c, d>0 \text { s.t. } c<\frac{1}{8 t} \text { and }|g(z)| \leqslant d e^{c|z|^{2}}\right\}
$$

Proposition 1.3.1. For every $g \in \mathcal{E}_{t}$ the Toeplitz operator $T_{g}^{t}$ is densely defined on $H_{t}^{2}$ and its domain of definition $D\left(T_{g}^{t}\right)$ contains the space of holomorphic polynomials $\mathbb{P}[z]$ as well as the linear space of the normalized kernels

$$
\mathcal{L}_{H_{t}^{2}}=\operatorname{span}\left\{k_{z}^{t}(w):=e^{\frac{1}{4 t} w \bar{z}} e^{-\frac{1}{8 t}|z|^{2}}\right\}_{z \in \mathbb{C}^{n}}
$$

Moreover, the correspondence $\mathcal{E}_{t} \ni g \longrightarrow T_{g}^{t}$ is one-to-one.

Proof. For $f \in \mathbb{P}[z] \cup \mathcal{L}\left(H_{t}^{2}\right)$ the multiplication operator $M_{f}$ by $f$ maps $\mathcal{E}_{t}$ into itself. Hence for any $g \in \mathcal{E}_{t}$ we have $M_{f} g=f g \in \mathcal{E}_{t} \subset L^{2}\left(\mathbb{C}^{n}, d \mu_{t}\right)$ which means $f \in D\left(T_{g}^{t}\right)$. Now suppose $T_{g}^{t}=0$ then for any $\alpha, \beta \in \mathbb{N}^{n}$ we have

$$
\left\langle g, z^{\alpha} \bar{z}^{\beta}\right\rangle_{t}=\left\langle g z^{\beta}, z^{\alpha}\right\rangle_{t}=\left\langle P^{t} g z^{\beta}, z^{\alpha}\right\rangle_{t}=\left\langle T_{g}^{t} z^{\beta}, z^{\alpha}\right\rangle_{t}=0
$$

However, the polynomials in $z$ and $\bar{z}$ are dense in $L^{2}\left(\mathbb{C}^{n}, d \mu_{t}\right)$ (c.f. $\left.[84,119]\right)$ and therefore $g=0$ a.e. on $\mathbb{C}^{n}$. 
Remark 1.3.1. A more general notion of Toeplitz operators on the Segal-Bargmann space $H^{2}:=H_{\frac{1}{4}}^{2}$ was introduced by J. Janas in $[117,118]$ (c.f. also Section 3.2). For a measurable function $g$, J. Janas defined the operator $\tilde{T}_{g}$ on the maximal domain $D\left(\tilde{T}_{g}\right):=\left\{f \in H^{2} \mid\right.$ $g f=h+r, h \in H^{2}$ and $\left.\int_{\mathbb{C}^{n}} r \bar{p} d \mu_{\frac{1}{4}}=0, \forall p \in \mathbb{P}[z]\right\}$ by $\tilde{T}_{g} f=h$. It turned out that this operator is well defined with $D\left(T_{g}\right) \subset D\left(\tilde{T}_{g}\right)$ and $T_{g}=\tilde{T}_{g}$ on $D\left(T_{g}\right)$. In [95], S. Grudsky and $N$. Vasilevski characterized a class of non-zero radial symbols $g$ on which $\tilde{T}_{g}$ is identically zero on the Segal-Bargmann space of the complex plane. According to Proposition 1.3.1, this class contains only symbols of high growth order at infinity. Explicit examples of such symbols where constructed by W. Bauer and T. Le in [16].

We also remark that if $g \in \mathcal{E}_{t}$ is radial in each component, $g(z)=g\left(\left|z_{1}\right|, \cdots,\left|z_{n}\right|\right)$, then by the rotation-invariant property of $\mu_{t}$ the Toeplitz operator $T_{g}^{t}$ is diagonal with respect to the standard orthonormal basis (1.3.6). Indeed, if we represent each component of $u=\left(u_{1}, \cdots, u_{n}\right) \in \mathbb{C}^{n}$ in its polar coordinates by $u_{j}=r_{j} \xi_{j}$, where $r_{j}=\left|u_{j}\right|$ and $\xi_{j} \in \mathbb{S}^{1}$ then we obtain

$$
\begin{aligned}
T_{g}^{t} z^{\alpha} & =\int_{\mathbb{C}^{n}} g(u) u^{\alpha} e^{\frac{1}{4 t} z \bar{u}} d \mu_{t}(u) \\
& =\sum_{\beta \in \mathbb{N}_{0}} \frac{z^{\beta}}{(4 t)^{|\beta|} \beta !} \int_{\mathbb{C}^{n}} g(u) u^{\alpha} \bar{u}^{\beta} d \mu_{t}(u) \\
& =\sum_{\beta \in \mathbb{N}_{0}} \frac{z^{\beta}}{(4 t)^{|\beta|} \beta !} \frac{1}{(4 \pi t)^{n}} \int_{0}^{\infty} g\left(r_{1}, \cdots, r_{n}\right) \prod_{j=1}^{n} r_{j}^{\alpha_{j}+\beta_{j}+1} e^{-\frac{r_{j}^{2}}{4 t}} d r_{j} \int_{\mathbb{S}^{1}} \xi_{j}^{\alpha_{j}} \bar{\xi}_{j}^{\beta_{j}} d \sigma\left(\xi_{j}\right) \\
& =\frac{z^{\alpha}}{(4 t)^{|\alpha|} \alpha !} \frac{1}{(2 t)^{n}} \int_{0}^{\infty} g\left(r_{1}, \cdots, r_{n}\right) \prod_{j=1}^{n} r_{j}^{\alpha_{j}+\beta_{j}+1} w_{t}\left(r_{j}\right) d r_{j} .
\end{aligned}
$$

Conversely, in [16] W. Bauer and T. Le proved that if $g \in \mathcal{E}_{t}$ is such that $T_{g}^{t}$ is diagonal with respect to the standard orthonormal basis then $g$ is radial in each component. Now let us consider two radial symbols $f, g \in \mathcal{E}_{t}$.

On the one hand, since $T_{f}^{t}$ and $T_{g}^{t}$ are diagonal operators their product $T_{g}^{t} T_{f}^{t}$ and $T_{f}^{t} T_{g}^{t}$ are well defined and commute on the space of holomorphic polynomials $\mathbb{P}[z]$. In Chapter 3 , we will prove a wider result namely if $f, g \in \mathcal{E}_{t}$ are $k$-quasi-homogeneous symbols (having a specific form) then the Toeplitz operators $T_{g}^{t}$ and $T_{f}^{t}$ also commute on $\mathbb{P}[z]$. For example, put $f(z)=\varphi(r) \xi^{p} \bar{\xi}^{q}$ and $g(z)=\psi(r) \xi^{p} \bar{\xi}^{q}$ where $\varphi, \psi \in \mathcal{E}_{t}$ and $p, q$ are orthogonal multi indices s.t. $|p|=|q|$ then $T_{f}^{t}$ and $T_{g}^{t}$ commute.

On the other hand, one may ask whether for a non-constant radial symbol $f \in \mathcal{E}_{t}$ and for an arbitrary $g \in \mathcal{E}_{t}$ the commuting condition between $T_{f}^{t}$ and $T_{g}^{t}$ implies that $g$ is radial in its components. In [16], it was proved that this is not true in general for $f, g \in \mathcal{E}_{t}$. However, if we assume $f$ and $g$ to be in the space $\operatorname{Sym}_{>0}\left(\mathbb{C}^{n}\right):=\cap_{t>0} \mathcal{E}_{t}$ and $f$ is non-trivial and radial then $T_{f}^{t}$ and $T_{g}^{t}$ commute if and only if $g\left(e^{i \theta} z\right)=g(z)$ for a.e. $\theta \in \mathbb{R}$ and a.e. $z \in \mathbb{C}^{n}$. This generalize the previously known result in [17] for the case $n=1$. If we allow the operator symbol $g$ to grow by high order at infinity and even in the case where $f$ is bounded then the implication , $g$ is radial" is no longer true (c.f. [17]). In Chapter 2, by studying commuting Toeplitz operators 
with quasi-homogeneous symbols we recover the examples in [17] of radial functions whose Toeplitz operators commute with another Toeplitz operator having a non-radial symbol.

In connection with the analysis of $T_{g}^{t}$ the heat semigroup:

$$
\tilde{g}^{t}(z):=g \star w_{t}(z)=\frac{1}{(4 \pi t)^{n}} \int_{\mathbb{C}^{n}} g(u) e^{-\frac{|z-u|^{2}}{4 t}} d v(u)
$$

plays an important role and is called the ,heat transform” of $g$ at time $t$ or the Berezin symbol of $T_{g}^{t}$. The use of the Berezin transform as a determining factor of the behavior of the Toeplitz operators on the Segal-Bargmann space has generated an extensive list of results [14-17, 35, $64,111,112]$. It should be noted that whenever $g$ is in the class of symbols:

$$
\mathcal{T}_{t}:=\left\{g: \mathbb{C}^{n} \longrightarrow \mathbb{C} \mid g k_{z}^{t} \in L^{2}\left(\mathbb{C}^{n}, d \mu_{t}\right) \text { for all } z \in \mathbb{C}^{n}\right\}
$$

the Berezin transform of $g$ coincide with the Berezin transform of the Toeplitz operator $T_{g}^{t}$ :

$$
\tilde{g}^{t}(z)=\left\langle T_{g}^{t} k_{z}^{t}, k_{z}^{t}\right\rangle_{t}=\widetilde{T_{g}^{t}}(z)
$$

Therefore, by the Propositions 1.1.4 and 1.3.1 for any $g \in \mathcal{E}_{t} \subset \mathcal{T}_{t}$ such that $T_{g}^{t}$ is bounded on $H_{t}^{2}$ the Berezin transform $\tilde{g}^{t}$ is real analytic and bounded by $\left\|T_{g}^{t}\right\|$ and satisfies $\tilde{g}^{t}=0$ if and only if $g=0$ a.e. on $\mathbb{C}^{n}$. More general, in [14] it has been proved that the Berezin transform on the space $\operatorname{Sym}_{>0}\left(\mathbb{C}^{n}\right)=\cap_{t>0} \mathcal{E}_{t}$ is one-to-one. In the next chapter, we shall use a similar argument to that in [14] together with Proposition 1.1.4 to prove this result on every space $\mathcal{E}_{t}$ (c.f. Remark 2.2.2). Note that, since $k_{z}^{t}$ converges to zero weakly on $H_{t}^{2}$ as $z \longrightarrow \infty$ equation (1.3.14) shows that the Berezin transform of $g$ vanishes at infinity $\left(\tilde{g}^{t} \in C_{0}\left(\mathbb{C}^{n}\right)\right)$ whenever the Toeplitz operator $T_{g}^{t}$ is compact on $H_{t}^{2}$.

Let us now study some properties of the Berezin transform and then apply it to study the behavior of the Toeplitz operator. First we remark that the convolution product $g \star w_{t}(z)$ may not exists for particular $z$ and $t$. However, if $g \in L^{2}\left(\mathbb{C}^{n}, d \mu_{s}\right)$ for some $s>0$ then the integral in (1.3.12) converges absolutely for every $t$ such that $t<2 s$. Indeed, for every $z \in \mathbb{C}^{n}$ the function $\psi_{z, t, s}(u):=(4 \pi s)^{n} w_{t}(z-u) e^{\frac{|u|^{2}}{4 s}} \in L^{2}\left(\mathbb{C}^{n}, d \mu_{s}\right)$ hence

$$
\left|g \star w_{t}(z)\right| \leq \int_{\mathbb{C}^{n}}|g(u)| w_{t}(z-u) d v(u)=\int_{\mathbb{C}^{n}}|g(u)| \psi_{z, t, s}(u) d \mu_{s}(u)<\infty .
$$

Moreover, for every $t<s$ the Berezin transform $\tilde{g}^{t} \in L^{2}\left(\mathbb{C}^{n}, d \mu_{s-t}\right)$ with $\left\|\tilde{g}^{t}\right\|_{s-t} \leq\|g\|_{s}$. In fact, using Cauchy-Schwartz inequality

$$
\begin{aligned}
\left|g \star w_{t}(z)\right|^{2} & \leq\left[\int_{\mathbb{C}^{n}}|g(u)| w_{2 t}^{2}(z-u) d v(u)\right]^{2} \\
& \leq \int_{\mathbb{C}^{n}} w_{t}(z-u) d v(u) \int_{\mathbb{C}^{n}}|g(u)|^{2} w_{t}(z-u) d v(u)=\int_{\mathbb{C}^{n}}|g(u)|^{2} w_{t}(z-u) d v(u) .
\end{aligned}
$$


Hence, by (1.3.9) we obtain

$$
\begin{aligned}
\left\|\tilde{g}^{t}\right\|_{s-t}^{2} & =\int_{\mathbb{C}^{n}}\left|\tilde{g}^{t}(z)\right|^{2} d \mu_{s-t}(z) \\
& \leq \int_{\mathbb{C}^{n}}|g(u)|^{2} w_{t}(z-u) d v(u) w_{s-t}(z) d v(z) \\
& =\int_{\mathbb{C}^{n}}|g(u)|^{2} w_{t} \star w_{s-t}(u) d v(u) \\
& =\int_{\mathbb{C}^{n}}|g(u)|^{2} w_{s}(u) d v(u)=\|g\|_{s}^{2} .
\end{aligned}
$$

From (1.3.9) again it follows that the Berezin transform of $g \in L^{2}\left(\mathbb{C}^{n}, d \mu_{s}\right)$ fulfills the semigroup identity:

$$
\tilde{g}^{(t+\nu)}(z)=g \star w_{t+\nu}(z)=g \star\left(w_{t} \star w_{\nu}\right)=\left(g \star w_{t}\right) \star w_{\nu}=\left\{\tilde{g}^{t}\right\}^{\sim \nu},
$$

for every $t, \nu>0$ such that $t+\nu<s$. This is not surprising since by direct calculations one can show that if $g \in L^{\infty}\left(\mathbb{C}^{n}\right)$ then the Berezin transform $u(x, t):=\tilde{g}^{t}$ is the solution of the heat equation $u(x, t)=e^{-t \Delta} g$ at any time $t$.

On the one hand, using (1.3.16) it is easy to see that if $g \in L^{2}\left(\mathbb{C}^{n}, d \mu_{s}\right)$ then

$$
\left\|\tilde{g}^{t}\right\|_{\infty} \leq\left\|\tilde{g}^{\nu}\right\|_{\infty}
$$

for every $0<\nu<t<2 s\left(\left|\tilde{g}^{t}(z)\right|=\left|\tilde{g}^{\nu} \star w_{t-\nu}(z)\right| \leq\left\|\tilde{g}^{\nu}\right\|\right)$. On the other hand, it is easily checked that $\left|\tilde{g}^{\nu}\right| \leq\left.\left(\frac{t}{\nu}\right)^{n} \widetilde{|g|}\right|^{t}$ for every $0<\nu<t<2 s$. This shows that, for $g \in L^{2}\left(\mathbb{C}^{n}, d \mu_{s}\right)$, $\left\|\left.\widetilde{\mid g}\right|^{t_{0}}\right\|_{\infty}<\infty$ for some fixed $0<t_{0}<2 s$ then $\left\|\tilde{g}^{t}\right\|_{\infty}<\infty$ for all $0<t<2 s$ (c.f. Proposition 2 in [35]).

Let us now collect some interesting results which relates the Berezin transform of a function and its corresponding Toeplitz operator. Using (1.3.14) and (1.3.17) we see that if $T_{g}^{\nu}$ is bounded (respectively compact) for some $0<\nu<2 s$ then $\tilde{g}^{t}$ is bounded (respectively $\tilde{g}^{t} \in C_{0}\left(\mathbb{C}^{n}\right)$ where $C_{0}\left(\mathbb{C}^{n}\right)$ is the space of functions vanishing at infinity) for every $0<\nu \leq t$. Moreover, in [35] and later on in [15] it has been proved that the norm of the Berezin transform is dominated by that of the corresponding Toeplitz operator in the following sense.

Theorem 1.3.1. [15, 35] For $g \in \mathcal{T}_{t}$ and every s such that $\frac{t}{2}<s<2 t$ there is a universal constant $c(t, s)>0$, independent of $g$, such that

$$
\left\|\tilde{g}^{s}\right\|_{\infty} \leq c(t, s)\left\|T_{g}^{t}\right\| .
$$

Therefore, $\tilde{g}^{s}$ is bounded for all $\frac{t}{2}<s<2 t$ whenever $T_{g}^{t}$ is bounded. Furthermore, the above theorem was used in [15] to prove an interesting property relating the compactness of the Toeplitz operator and the vanishing of the Berezin transform at infinity. For completeness we state the result and give the proof (c.f. Theorem 4 in [15]).

Theorem 1.3.2. [15] Let $g \in \mathcal{T}_{t}$. If $T_{g}^{t}$ is compact on $H_{t}^{2}$ for some $t>0$ then $\tilde{g}^{s} \in C_{0}\left(\mathbb{C}^{n}\right)$ for all $\frac{t}{2}<s<2 t$. 
Proof. Suppose $T_{g}^{t}$ is compact then by Theorem 1.2.2 there is a sequence of compactly supported continuous functions $\left(g_{j}\right)_{j}$ such that $\left\|T_{g}^{t}-T_{g_{j}}^{t}\right\| \stackrel{j \rightarrow \infty}{\longrightarrow} 0$. Therefore, by (1.3.18) for any fixed $s$ such that $\frac{t}{2}<s<2 t$ we obtain $\left\|\tilde{g}^{s}-\widetilde{g}_{j}{ }^{s}\right\|_{\infty} \leq c(t, s)\left\|T_{g}^{t}-T_{g_{j}}^{t}\right\| \stackrel{j \rightarrow \infty}{\longrightarrow} 0$. Since each $\widetilde{g}_{j}^{s} \in C_{0}\left(\mathbb{C}^{n}\right)$ and is of compact support, then $\tilde{g}^{s} \in C_{0}\left(\mathbb{C}^{n}\right)$.

A converse implication to the above theorem was also proved in [15]. Roughly speaking, if $g \in \mathcal{T}_{t}$ such that $\tilde{g}^{s} \in C_{0}\left(\mathbb{C}^{n}\right)$ for some $0<s<\frac{t}{2}$ then $T_{g}^{t}$ is compact. The proof uses some known pseudo-differential estimates. More clearly, it is well known [12, 84, 98] that via the Bargmann transform,

$$
\beta: L^{2}\left(\mathbb{R}^{n}, d x\right) \longrightarrow H_{t}^{2}
$$

defined by $[\beta f](z)=2^{\frac{n}{4}} \int_{\mathbb{R}^{n}} f(x) e^{\sqrt{\frac{\pi}{t}} x z-\pi x^{2}-\frac{z^{2}}{8 t}} d x$, every Toeplitz operator $T_{g}^{t}$ on $H_{t}^{2}$ is unitary equivalent to the Weyl-pseudodifferential operator $W_{\tilde{g}^{\frac{t}{2}}}$ acting on $L^{2}\left(\mathbb{R}^{n}, d x\right)$. Since $\tilde{g}^{\frac{t}{2}} \in$ $C_{0}\left(\mathbb{C}^{n}\right)$ an application of Pool's theorem [143] together with the Calderon-Vaillancourt theorem [84] proves that $W_{\tilde{g}^{\frac{t}{2}}}$ is compact on $L^{2}\left(\mathbb{R}^{n}, d x\right)$.

These results together with the equivalence between the compactness of $T_{g}^{t}$ and the vanishing at infinity of $\tilde{g}^{t}$ whenever $g$ is of bounded mean oscillation (c.f. [64]) were used in [15] to show the independence of the compactness of $T_{g}^{t}$ with respect to the time $t$. In Chapter 5 , we will use similar techniques to prove that the compactness of Toeplitz operator (with bounded symbols) acting on the Bergman space over a bounded symmetric domain is independent of the standard weights and results similar to Theorems 1.3.1 and 1.3.2 are obtained.

\subsection{Bergman spaces over the unit ball of $\mathbb{C}^{n}$}

The weighted and the ,unweighted” Bergman spaces over the unit ball of $\mathbb{C}^{n}$ and related operators have been studied intensively by several authors [3, 4, 10, 11, 72, 101, 125, 185-187]. In this section, we introduce some properties of these RKHS and compare the situation to the case of the Segal-Bargmann space.

Let $\Omega=\mathbb{B}^{n}$ be the open unit ball in $\mathbb{C}^{n}$ and for any $\lambda>-1$ consider the radial weight

$$
w_{\lambda}(z):=c_{\lambda}\left(1-|z|^{2}\right)^{\lambda}
$$

where the normalizing constant $c_{\lambda}:=\frac{(n+\lambda) !}{\pi^{n} \lambda !}$ is chosen so that $d \mu_{\lambda}(z):=w_{\lambda}(z) d v(z)$ is a probability measure on $\mathbb{B}^{n}$. Theses weights $\left\{w_{\lambda}\right\}_{\lambda>-1}$ are the standard weights on $\mathbb{B}^{n}$. In fact, they arise from the Jordan triple determinant polynomial $h(z, w)=1-z \bar{w}$ associated to symmetric domain $\mathbb{B}^{n}$ (c.f. Chapter 5).

Denote by $A_{\lambda}^{2}\left(\mathbb{B}^{n}\right)$ the weighted Bergman space in $L_{\lambda}^{2}:=L^{2}\left(\mathbb{B}^{n}, d \mu_{\lambda}\right)$. When the weight $\lambda=0$, we simply write $A^{2}\left(\mathbb{B}^{n}\right)$ instead of $A_{0}^{2}\left(\mathbb{B}^{n}\right)$ and refer to $A^{2}\left(\mathbb{B}^{n}\right)$ as the ,, unweighted" Bergman space over the unit ball. The inner product and the norm on $L_{\lambda}^{2}$ or $A_{\lambda}^{2}\left(\mathbb{B}^{n}\right)$ is denoted by $\langle\cdot, \cdot\rangle_{\lambda}$ and $\|\cdot\|_{\lambda}$, respectively. 
By Theorem 1.2.1 we know that $A_{\lambda}^{2}\left(\mathbb{B}^{n}\right)$ is a reproducing kernel Hilbert space. Moreover, by integration in polar coordinates and using (1.3.5) it is easy to check that the set

$$
\left\{e_{\alpha}(z):=\sqrt{\frac{\Gamma(n+|\alpha|+\lambda+1)}{\alpha ! \Gamma(n+\lambda+1)}} z^{\alpha}\right\}_{\alpha \in \mathbb{N}^{n}},
$$

where $\Gamma$ stands for the Gamma function, forms an orthonormal basis of $A_{\lambda}^{2}\left(\mathbb{B}^{n}\right)$. Therefore, by (1.2.3) the reproducing kernel of $A_{\lambda}^{2}$, denoted by $K^{\lambda}$, is given by the series

$$
K^{\lambda}(u, z)=\sum_{\alpha \in \mathbb{N}^{n}} \frac{\Gamma(n+|\alpha|+\lambda+1)}{\alpha ! \Gamma(n+\lambda+1)} u^{\alpha} \bar{z}^{\alpha}=\frac{1}{(1-u \bar{z})^{n+1+\lambda}} .
$$

According to (1.2.4) the orthogonal projection $P^{\lambda}$ from $L_{\lambda}^{2}$ onto $A_{\lambda}^{2}\left(\mathbb{B}^{n}\right)$ is given by the integral formula

$$
\left[P^{\lambda} h\right](z)=\left\langle h, K^{\lambda}(\cdot, z)\right\rangle_{\lambda}=\int_{\mathbb{B}^{n}} \frac{h(u)}{(1-z \bar{u})^{n+\lambda+1}} d \mu_{\lambda}(u), \quad h \in L_{\lambda}^{2}, z \in \mathbb{B}^{n}
$$

Note that, for fixed $z \in \mathbb{B}^{n}$ the reproducing kernel $K(u, z)$ is bounded in $u$ and therefore $P^{\lambda}$ can be extended to $L_{\lambda}^{1}:=L^{1}\left(\mathbb{B}^{n}, d \mu_{\lambda}\right)$ by its integral representation in (1.4.1). Therefore, for a function $g \in L_{\lambda}^{1}$ the Toeplitz operator $T_{g}^{\lambda}$, given by

$$
T_{g}^{\lambda} h(z)=\left[P^{\lambda} g h\right](z)=\int_{\mathbb{B}^{n}} \frac{h(u) g(u)}{(1-z \bar{u})^{n+\lambda+1}} d \mu_{\lambda}(u),
$$

is densely defined on $A_{\lambda}^{2}$ and its domain of definition $D\left(T_{g}^{\lambda}\right)$ contains all bounded holomorphic functions on $\mathbb{B}^{n}$. Since $P^{\lambda}$ is not bounded on $L_{\lambda}^{1}$ the operator $T_{g}^{\lambda}$ is unbounded in general.

The Berezin transform of a function $g \in L_{\lambda}^{1}$, denoted $\tilde{g}^{\lambda}$, is defined to be the Berezin transform of the Toeplitz operator $T_{g}^{\lambda}$ :

$$
\tilde{g}^{\lambda}(z):=\left\langle T_{g}^{\lambda} k_{z}^{\lambda}, k_{z}^{\lambda}\right\rangle=\left\langle g k_{z}^{\lambda}, k_{z}^{\lambda}\right\rangle=\left(1-|z|^{2}\right)^{n+\lambda+1} \int_{\mathbb{B}^{n}} \frac{g(u)}{|1-u \bar{z}|^{2(n+\lambda+1)}} d \mu_{\lambda}(u),
$$

where $k_{z}^{\lambda}$ is the normalizing kernel function given by $k_{z}^{\lambda}(u)=\left(1-|z|^{2}\right)^{\frac{1}{2}(n+\lambda+1)} \frac{1}{(1-u \bar{z})^{n+1+\lambda}}$. Note that, by the above definition the Berezin transform $\tilde{g}^{\lambda}$ replaces the heat transform in the case of the Segal-Bargmann space. Since the mapping $g \longrightarrow T_{g}^{\lambda}$ is bounded on $L^{\infty}\left(\mathbb{B}^{n}\right)$ it follows that the Berezin transform $B_{\lambda}: g \longrightarrow \tilde{g}^{\lambda}$ is bounded on $L^{\infty}\left(\mathbb{B}^{n}\right)$ with $\left\|\tilde{g}^{\lambda}\right\|_{\infty} \leq\|g\|_{\infty}$. Moreover, in [125] it has been shown that the mapping $B_{\lambda}$ is bounded on $L^{p}\left(\mathbb{B}^{n}, d \mu_{\lambda}\right)$ if and only if $p>1$. Note that $B_{\lambda}$ is one-to-one on $L_{\lambda}^{1}$ (c.f. p. 32 in [101] for the case $n=1$ ). Indeed, suppose $\tilde{g}^{\lambda}=0$ for some $g \in L_{\lambda}^{1}$ then the function

$$
G(z):=\frac{\tilde{g}^{\lambda}(z)}{\left(1-|z|^{2}\right)^{n+\lambda+1}}=\int_{\mathbb{B}^{n}} \frac{g(u)}{(1-u \bar{z})^{(n+\lambda+1)}}(1-u \bar{z})^{(n+\lambda+1)} d \mu_{\lambda}(u)=0 .
$$

Therefore, for any $\alpha, \beta \in \mathbb{N}_{0}^{n}$ we have $\left(\partial_{z}^{\alpha} \partial_{\bar{z}}^{\beta} G\right)(0)=0$, where $\partial_{z}^{\alpha}:=\partial_{z_{1}}^{\alpha_{1}} \partial_{z_{2}}^{\alpha_{2}} \cdots \partial_{z_{n}}^{\alpha_{n}}$. Differentiation under the integral sign shows that $\int_{\mathbb{B}^{n}} \bar{u}^{\alpha} u^{\beta} g(u) d \mu_{\lambda}(u)=0$ hence $g=0$ a.e. on $\mathbb{B}^{n}$. 
There is another useful way to write the Berezin transform as an integral involving the involutive automorphisms $\varphi_{z}$ of the ball. For each $z \in \mathbb{B}^{n}$ the function $\varphi_{z}$ is the automorphism $\mathbb{B}^{n}$ which exchanges 0 and $z$ and is given by

$$
\varphi_{z}(u)=\frac{z-P_{z}(u)-\sqrt{1-|z|^{2} Q(u)}}{1-u \bar{z}} \quad u \in \mathbb{B}^{n}
$$

where $P_{z}$ is the orthogonal projection from $\mathbb{C}^{n}$ on to the one-dimensional subspace generated by $z$ and $Q(u)=u-P_{z}(u)$. Remark that the automorphism group of the ball $\operatorname{Aut}\left(\mathbb{B}^{n}\right)$ is generated by the unitary operators on $\mathbb{C}^{n}$ and the involutions $\left\{\varphi_{z}\right\}_{z \in \mathbb{B}^{n}}$ (c.f. Theorem 2.2.5 in [149]). Moreover, we have

$$
1-\left|\varphi_{z}(u)\right|^{2}=\frac{\left(1-|z|^{2}\right)\left(1-|z|^{2}\right)}{\left.|1-u \bar{z}|^{2}\right)}
$$

and

$$
J_{R} \varphi_{z}(u)=\left(\frac{1-|z|^{2}}{|1-u \bar{z}|^{2}}\right)^{n+1}, \quad \text { where } J_{R} \varphi_{z} \text { is the real Jacobian of } \varphi_{z}
$$

Therefore, by the change of variable $u \longrightarrow \varphi_{z}(u)$ together with (1.4.3) and (1.4.4) we obtain:

$$
\begin{aligned}
\tilde{g}^{\lambda}(z) & =\int_{\mathbb{B}^{n}} \frac{g(u)\left(1-|z|^{2}\right)^{n+\lambda+1}}{|1-u \bar{z}|^{2(n+\lambda+1)}} d \mu_{\lambda}(u) \\
& =\int_{\mathbb{B}^{n}} g \circ \varphi_{z}(u) d \mu_{\lambda}(u) .
\end{aligned}
$$

Let us choose a function $g \in L_{\lambda}^{1}$ for all $-1<\lambda \leq 0$ such that the Berezin transform satisfies the semigroup property $\left(\widetilde{\tilde{g}}^{\nu}=\tilde{g}^{\nu+s}\right)$. Then by (1.4.5) we have

$$
\widetilde{\tilde{g}}^{\lambda}(z)=\int_{\mathbb{B}^{n}} \tilde{g}^{\lambda} \circ \varphi_{z}(u) d \mu_{\lambda}(u)=\tilde{g}^{\lambda}(z)=\int_{\mathbb{B}^{n}} g \circ \varphi_{z}(u) d \mu_{\lambda}(u) .
$$

Since the Berezin transform map is one-to-one on $L_{\lambda}^{1}$ we see that $\widetilde{\tilde{g}}^{0}=\tilde{g}^{\lambda}$ if and only if $g=\tilde{g}^{\lambda}$ (compare the situation to the Segal-Bargmann case). Therefore, it is natural to study the fix points of the Berezin transform. In fact, the problem of characterizing the functions invariant under the Berezin transform has been encountered by several authors [2, 8, 75, 137]. In the case $n=1$, and under the assumption that $g$ is continuous up to the boundary $g$ is a fix point of the Berezin transform if and only if it is a harmonic function (c.f. Prop. 6.20 in [186]). This result is still true if we assume only that $\mathrm{g}$ is bounded on the open unit disc [75].

We aim now to prove that the M-harmonic functions on $\mathbb{B}^{n}$ are fixed points of the Berezin transform. Recall that a twice differentiable function $g$ is called M-harmonic if $(\tilde{\Delta} g)(z):=$ $\Delta\left(g \circ \varphi_{z}\right)(0)=0$ for all $z \in \mathbb{B}^{n}$. In case $n=1$, they are precisely the harmonic functions and this fails to be true for $n>1$ (c.f. Remark 4.1.4 in [149]).

Let $g$ be M-harmonic function then by the mean value property of the M-harmonic functions (Theorem 4.1.3 in [149]) we can write 


$$
g\left(\varphi_{z}(0)\right)=\frac{1}{\sigma\left(\mathbb{S}^{2 n-1}\right)} \int_{\mathbb{S}^{2 n-1}} g\left(\varphi_{z}(r \xi)\right) d \sigma(\xi), \quad \text { for every } 0<r<1 .
$$

Multiplying both sides of the above equation by a factor of $2 n r^{2 n-1}\left(1-r^{2}\right)^{\lambda}$ and integrating over $[0,1)$ we obtain

$$
2 n g(z) \int_{0}^{1} r^{2 n-1}\left(1-r^{2}\right)^{\lambda} d r=\frac{1}{\sigma\left(\mathbb{S}^{2 n-1}\right)} \int_{\mathbb{B}^{n}} g \circ \varphi_{z}(u)\left(1-|u|^{2}\right) d v(u) .
$$

Therefore,

$$
g(z)=\int_{\mathbb{B}^{n}} g \circ \varphi_{z}(u) d \mu_{\lambda}(u)=\tilde{g}^{\lambda}(z) .
$$

By the above calculations it should be noted that any function $g \in L_{\lambda}^{1}$ is a fix point for the Berezin transform $B_{\lambda}$ if and only if $g$ satisfies the mean value property . In case of $\lambda=0$, it has been proved in [2] that functions in $L_{0}^{1}$ which satisfy the mean value property are exactly those M-harmonic in case $n \leq 11$ and this fails for higher dimensions. Therefore there are fix points of the Berezin transform $B_{0}$ which are not M-harmonic, and according to Proposition 13.4.4 in [149] these functions do not admit continuous extension to the closed ball $\overline{\mathbb{B}^{n}}$.

Similar to the case of the Segal-Bargmann space it is clear that if $g \in L_{\lambda}^{1}$ then $\left\|\tilde{g}^{\lambda_{0}}\right\|_{\infty} \leq$ $\left\|\tilde{g}^{\lambda}\right\|_{\infty} \leq\left\|T_{g}^{\lambda}\right\|$ for all $\lambda_{0} \geq \lambda$. Therefore, if $T_{g}^{\lambda}$ is bounded then the Berezin transform $\tilde{g}^{\lambda_{0}}$ is bounded for all $\lambda_{0} \geq \lambda$. Moreover, since $k_{z}^{\lambda} \stackrel{z \rightarrow \partial \mathbb{B}^{n}}{\longrightarrow} 0$ weakly $\tilde{g}^{\lambda_{0}} \in C_{0}\left(\mathbb{B}^{n}\right)$ whenever $T_{g}^{\lambda}$ is compact for some $\lambda \leq \lambda_{0}$. Furthermore, if we suppose that $g$ is M-harmonic then $T_{g}^{\lambda}$ is bounded (respectively compact) if and only if $g$ is bounded (respectively $g=0$ ). Indeed, since the M-harmonic functions are fix points of the Berezin transform $T_{g}^{\lambda}$ is bounded if and only if $g=\tilde{g}^{\lambda}$ is bounded. Now, if we suppose that $T_{g}^{\lambda}$ is compact then $g=\tilde{g}^{\lambda} \stackrel{z \rightarrow \partial \mathbb{B}^{n}}{\longrightarrow} 0$ and therefore by the maximum principle of the M-harmonic functions (c.f. Theorem 4.3.2 in [149]) it follows that $g=0$.

The connection between the vanishing of the Berezin transform of the function near the boundary and the compactness of its corresponding Toeplitz operator has been studied frequently [10, 158, 183, 187]. In [187] it was proved that for symbols $g$ of bounded mean oscillation $\tilde{g}^{0} \in C_{0}\left(\mathbb{B}^{n}\right)$ if and only if $T_{g}^{0}$ is compact on $A^{2}\left(\mathbb{B}^{n}\right)$. Later on in [15] and as an analogue to the Segal-Bargmann space case, the existence of a universal constant $c\left(\lambda, \lambda_{0}\right)$ was shown such that

$$
\left\|\tilde{g}^{\lambda}\right\|_{\infty} \leq c\left(\lambda, \lambda_{0}\right)\left\|T_{g}^{\lambda}\right\|
$$

for $g$ in a suitable class of symbols. This estimation together with the result [187] were used in [15] to prove that the compactness of the Toeplitz operator $T_{g}^{\lambda}$ is independent of the weight $\lambda$ whenever $g$ is of bounded mean oscillation. 


\section{Chapter 2}

\section{Commuting Toeplitz operators with quasi-homogeneous symbols on the Segal-Bargmann space}

For the Gaussian measure $\mu$ on the complex plane $\mathbb{C}$ given by the density $d \mu:=d \mu_{\frac{1}{4}}=$ $\frac{1}{\pi} e^{-|\cdot|^{2}} d v$, where $v$ is the usual Lebesgue measure, we denote by $H^{2}:=H_{\frac{1}{4}}^{2}=H^{2}(\mathbb{C}, d \mu)$ the Segal-Bargmann space over $\mathbb{C}$. As introduced in the previous chapter $H^{2}$ is a closed subspace of $L^{2}:=L^{2}(\mathbb{C}, d \mu)$ consisting of all $\mu$-square integrable entire functions on $\mathbb{C}$. We know that $H^{2}$ is a RKHS with the reproducing kernel $K(w, z)=e^{w \bar{z}}$. In particular, if $P$ denotes the orthogonal projection of $L^{2}$ onto $H^{2}$ then

$$
[P h](z)=\langle h, K(\cdot, z)\rangle=\int_{\mathbb{C}} h(w) e^{z \bar{w}} d \mu(w), \quad \forall z \in \mathbb{C}
$$

where $\langle\cdot, \cdot\rangle$ denotes the usual inner product on $L^{2}$.

For a function $g \in \mathcal{E}:=\mathcal{E}_{\frac{1}{2}} \subset \mathcal{E}_{\frac{1}{4}}$ (c.f. Definition 1.3.1), the Toeplitz operator $T_{g}:=T_{g}^{\frac{1}{4}}$ is given by

$$
T_{g}: D\left(T_{g}\right):=\left\{h \in H^{2} \mid g h \in L^{2}(\mathbb{C}, d \mu)\right\} \subset H^{2} \longrightarrow H^{2}: h \mapsto P(g h) .
$$

According to Proposition 1.3.1 the operator $T_{g}$ is densely defined and $D\left(T_{g}\right)$ contains the space of holomorphic polynomials $\mathbb{P}[z]$ as well as the linear space

$$
\mathcal{L}:=\mathcal{L}_{H^{2}}=\operatorname{span}\left\{k_{z}(w):=K(w, z) e^{-\frac{1}{2}|z|^{2}}\right\}_{z \in \mathbb{C}}
$$

In this chapter we are motivated by the following problem: Let $T=T_{z^{l} \bar{z}^{k}}$ with $l, k \in$ $\mathbb{N}_{0}$ be a Toeplitz operator with monomial symbol acting on $H^{2}$. Determine the symbols $\Psi$ of polynomial growth at infinity such that $T_{\Psi}$ and $T_{z^{l} \bar{z}^{k}}$ commute on the space of all holomorphic polynomials in $H^{2}$. By using polar coordinates we represent $\Psi$ as an infinite sum $\Psi\left(r e^{i \theta}\right)=\sum_{j=-\infty}^{\infty} \Psi_{j}(r) e^{i j \theta}$. Then we are able to reduce the above problem to the case of quasi-homogeneous symbols $\Psi=\Psi_{j} e^{i j \theta}$. We obtain the radial part $\Psi_{j}(r)$ in terms of the inverse 
Mellin transform of an expression which is a product of Gamma functions and a trigonometric polynomial. If we allow operator symbols of higher growth at infinity (but remaining in $\mathcal{E}$ ), we point out that in some of the cases more than one Toeplitz operator $T_{\Psi_{j} e^{i j \theta}}$ exists commuting with $T$.

The following section describes this problem in details as well as the steps used for solving it. We also state two of our main results which we prove throughout the chapter.

\subsection{Introduction}

The problem of characterizing the symbols of commuting Toeplitz operators on (weighted) Bergman spaces over various domains as well as the study of algebras of commuting Toeplitz operators, has attracted the interest of several authors $[8,9,16,17,60,61,66,126,129,130$, $144,168,169,172]$. The analysis often is restricted to the case where at least one of the symbols belongs to a certain subclass of functions.

On the Hardy space $H^{2}\left(\mathbb{S}^{1}\right)$, Brown and Halmos [54] were the first to obtain a complete description of bounded symbols so that the corresponding Toeplitz operators commute on $H^{2}\left(\mathbb{S}^{1}\right)$. In fact, they proved that two Toeplitz operators with bounded symbols commute on $H^{2}\left(\mathbb{S}^{1}\right)$ if and only if both symbols are analytic, or both are conjugate analytic, or one of them is a linear function of the other. An analogous problem in the case of the Bergman space $A(\mathbb{D})$ over the unit disc $\mathbb{D}$ was considered by S. Axler and Z. Čučković in [8]. Roughly speaking, they showed that for a pair of commuting Toeplitz operators on $A(\mathbb{D})$ with bounded harmonic symbols the result obtained by Brown and Halmos in [54] is still true. Of course, harmonicity is essential since any two Toeplitz operators with radial symbols commute on $A(\mathbb{D})$. Later on, with Rao [9], they proved that if two Toeplitz operators on $A(\mathbb{D})$ commute and ,, the symbol of one of them is non constant and analytic then the other is analytic too" [9].

Let $\varphi$ be a monomial or more generally a bounded quasi-homogeneous function on $\mathbb{D}$. In $[66,130]$ a complete characterization of the Toeplitz operator $T_{\psi}$ on $A(\mathbb{D})$ with bounded symbol $\psi$ was given such that $T_{\psi}$ commutes with $T_{\varphi}$. More precisely, every bounded function $\psi$ can be represented by an $L^{2}(\mathbb{D}, d v)$-convergent series $\psi\left(r e^{i \theta}\right)=\sum_{j=-\infty}^{\infty} \psi_{j}(r) e^{i j \theta}$ where $\psi_{j}(r)$ are bounded functions defined on the interval $[0,1$ ) ( here $r$ and $\theta$ represent the polar coordinated in $\mathbb{C}$ ). Using this fact, Z. Cučcković and N. V. Rao deduced that $T_{\psi}$ commutes with $T_{\varphi}$ commute if and only if for each $j \in \mathbb{Z}$ the Toeplitz operator $T_{\psi_{j}(r) e^{i j \theta}}$ commutes with $T_{\varphi}$. Then they were able to describe each function $\psi_{j}$ as the inverse Mellin transform of a product of Gamma functions (c.f. Theorem A0).

An analogous problem in the case of the Segal-Bargmann space and considering $\psi$ to be of polynomial growth at infinity is investigated in this chapter (for a detailed description of the problem c.f. below). In order to compare our results to those in the case of the Bergman space over the unit disc and for the sake of completeness we state the main result in [66].

Theorem A0. [66] Let $\psi\left(r e^{i \theta}\right)=\sum_{j=-\infty}^{\infty} \psi_{j}(r) e^{i j \theta}$ and $\varphi\left(r e^{i \theta}\right)=r^{m} e^{i \delta \theta}$ be bounded functions on $\mathbb{D}$, where $\psi_{j}$ are bounded, $m \in \mathbb{N}_{0}$ and $\delta \in \mathbb{N}$. Then $T_{\varphi} T_{\psi}=T_{\psi} T_{\varphi}$ on $A(\mathbb{D})$ if and 
only if for each $j \in \mathbb{Z}$ there is a constant $a_{j}$ such that

$$
\psi_{j}(r)=a_{j} M^{-1}\left[\frac{\Gamma\left(\frac{z+j}{2 \delta}\right) \Gamma\left(\frac{z+m+\delta-j}{2 \delta}\right)}{\Gamma\left(\frac{z+2 \delta-j}{2 \delta}\right) \Gamma\left(\frac{z+m+\delta+j}{2 \delta}\right)}\right](r),
$$

where $M^{-1}$ denotes the inverse Mellin transform.

Moreover, it was shown in [66] that for bounded functions $\Phi_{0}$ and $\Phi$, where $\Phi_{0}$ is nonconstant and radial (i.e. $\varphi\left(r e^{i \theta}\right)=\varphi(r)$ ), the operators $T_{\Phi_{0}}$ and $T_{\Phi}$ commute if and only if $\Phi$ is radial.

In the case of the Segal-Bargmann space it was recently shown in $[16,17]$ that the growth of symbols near infinity essentially influences the results. On the one hand, if $T_{\varphi_{1}}$ and $T_{\varphi_{2}}$ commute such that both symbols are of polynomial growth and one of them is radial nonconstant, then the other symbol must be radial, too. On the other hand, examples of commuting operators $T_{\varphi_{1}}$ and $T_{\varphi_{2}}$ exist where $\varphi_{1}$ is radial of exponential growth at infinity and $\varphi_{2}$ is nonradial and bounded, c.f. Example 2.7.3 of Section 7 and Example 5.6 in [17].

In this chapter, we fix a monomial $f_{m, \delta}\left(r e^{i \theta}\right)=r^{m} e^{i \delta \theta}$, where $m=l+k$ and $\delta=l-k$ with $l, k \in \mathbb{N}_{0}$. We search for functions $\Psi$ of polynomial growth at infinity such that $T_{f_{m, \delta}}$ and $T_{\Psi}$ commute as operators on the space of holomorphic polynomials. Analogous to the case of the Bergman space over the unit disc, we express $\Psi$ in a form of an $L^{2}$-convergent series of quasihomogeneous functions $\Psi\left(r e^{i \theta}\right)=\sum_{j=-\infty}^{\infty} \Psi_{j}(r) e^{i j \theta}$. It turns out, as in the case considered by Z. Cučković and N. V. Rao in [66], that it is sufficient to consider this problem for the operators $T_{f_{m, \delta}}$ and $T_{\Psi_{j} e^{i j \theta}}$. More precisely:

(1) Under the assumption $\left[T_{f_{m, \delta}}, T_{\Psi_{j} e^{i j \theta}}\right]:=T_{f_{m, \delta}} T_{\Psi_{j}} e^{i j \theta}-T_{\Psi_{j} e^{i j \theta}} T_{f_{m, \delta}}=0$, we characterize the functions $\Psi_{j}$ as the inverse Mellin transform of an expression formed up of Gamma functions and a trigonometric polynomial.

(2) For each fixed $j \in \mathbb{Z}$, we give a collection of quasi-homogeneous symbols $\Psi_{j} e^{i j \theta}$ such that the commutator $\left[T_{f_{m, \delta}}, T_{\Psi_{j} e^{i j \theta}}\right]$ vanishes.

The main idea in (1) is to derive a functional equation for the Mellin transform of $\Psi_{j} e^{-r^{2}}$ on some right half plane together with additional conditions starting from the relations

$$
\left[T_{f_{m, \delta}}, T_{\Psi_{j} e^{i j \theta}}\right]\left(z^{k}\right)=0, \quad \text { for all } \quad k \in \mathbb{N}_{0}
$$

We then, construct all possible solutions under the assumption that $\Psi$ has polynomial growth at infinity. Finally, we obtain $\Psi_{j}$ via the inverse Mellin transform.

In (2) we use the symbols obtained in (1) and study their growth behavior, to find the candidates of quasi-homogeneous symbols $\Psi_{j} e^{i j \theta}$ such that $\left[T_{f_{m, \delta}}, T_{\Psi_{j} e^{i j \theta}}\right]=0$. We prove that, for $j \in \mathbb{Z}$ sufficiently large, there exists at least one symbol $\Psi_{j}$ with this property having at 
most polynomial growth at infinity. Moreover, we point out that in general there are additional symbols $\Psi_{j}$ of exponential growth such that $T_{f_{m, \delta}}$ and $T_{\Psi_{j} e^{i j \theta}}$ commute.

We show that it is sufficient to treat only the case $\delta \geqslant 0$. Then we decompose the problem into three parts: (1) case $j>\delta$ (2) case $j<0$, (3) $0 \leqslant j \leqslant \delta$.

Now we state two of our main results. The first one addresses the problem (1)

Theorem A1. Let $\Psi$ be a measurable complex valued function on $\mathbb{C}$ of polynomial growth at infinity. We write $\Psi\left(r e^{i \theta}\right)=\sum_{j=-\infty}^{\infty} \Psi_{j}(r) e^{i j \theta}$ as an expansion in $L^{2}(\mathbb{C}, d \mu)$. For each $j>\delta$, we define a holomorphic function $G_{j}(z)$ for $\operatorname{Re}(z)>-j-1$ by

$$
G_{j}(z):=\prod_{l=1}^{j} \Gamma\left(\frac{z+p+l}{\delta}\right) \prod_{l=1}^{j-\delta}\left[\Gamma\left(\frac{z+\delta+l}{\delta}\right)\right]^{-1} \delta^{z},
$$

where $p:=\frac{\delta+m}{2}$. Suppose that $T_{f_{m, \delta}} T_{\Psi}=T_{\Psi} T_{f_{m, \delta}}$. Then for each $j>\delta$ there exists a trigonometric polynomial $p(z)=\sum_{|l|<\frac{\delta}{4}} a_{l} e^{\frac{2 \pi i l z}{\delta}}$ such that

$$
\Psi_{j}(r)=2 M^{-1}\left[G_{j}(z) p(z)\right]\left(r^{2}\right) r^{-j-2} e^{r^{2}},
$$

where $M^{-1}$ denotes the inverse Mellin transform.

On the other hand we have:

Theorem A2. Let $f_{m, \delta}\left(r e^{i \theta}\right)=r^{m} e^{i \delta \theta}$ be a monomial, and let $l \in \mathbb{Z}$ such that $|l|<\frac{\delta}{2 \pi} \arccos \frac{3}{4}$. Then for each $j>\delta$ the function

$$
\varphi_{j}(r) e^{i j \theta}:=M^{-1}\left[G_{j}(z) e^{\frac{2 \pi i l z}{\delta}}\right]\left(r^{2}\right) r^{-j-2} e^{r^{2}} e^{i j \theta},
$$

defines an operator symbol such that the commutator $\left[T_{f_{m, \delta}}, T_{\varphi_{j} e^{i j \theta}}\right]$ is well defined and vanishing on the space of holomorphic polynomials. Moreover, $\varphi_{j}(r) e^{i j \theta}$ is of polynomial growth at infinity in case $l=0$.

Results analogous to Theorems A1 and A2 and in the cases $j<0$ and $0 \leqslant j \leqslant \delta$ are also obtained (c.f. Theorems 2.5.1-2.6.1 and Corollary 2.6.1).

The chapter is organized as follows. In Section 2.2 we setup the notations and give some standard results used in our work. In particular, we make clear that the operator products $T_{f_{m, \delta}} T_{\Psi_{j} e^{i j \theta}}$ and $T_{\Psi_{j} e^{i j \theta}} T_{f_{m, \delta}}$ are well defined on a dense subset of $H^{2}$ containing the holomorphic polynomials and we reduce the problem to the case $\delta \geq 0$. Section 2.3 is devoted to the proof that $\left[T_{f_{m, \delta}}, T_{\Psi}\right]=0$ if and only if $\left[T_{f_{m, \delta}}, T_{\Psi_{j} e^{i j \theta}}\right]=0$ for all $j \in \mathbb{Z}$. Moreover, we give further equivalent conditions involving the Mellin transform of each $\left\{\Psi_{j}\right\}_{j \in \mathbb{Z}}$ at specific points. In Section 2.4 we consider the case $j>\delta$ and under the assumption that $\Psi$ is of at most polynomial growth at infinity and $\left[T_{f_{m, \delta}}, T_{\Psi_{j} e^{i j \theta}}\right]=0$, we derive a functional equation for the Mellin transform of $\Psi_{j} e^{-r^{2}}$ on some right half plane. Moreover, for an infinite number 
of indices $j \in \mathbb{Z}$ we prove that there is exactly one function $\Psi_{j}(r)$ (up to multiplication by a constant) of polynomial growth such that $\left[T_{f_{m, \delta}}, T_{\Psi_{j} e^{i j \theta}}\right]=0$. As an application we show that in case $m \neq 0$ the condition $\left[T_{f_{m, 0}}, T_{\Psi}\right]=0$ is equivalent to $\Psi$ being radial. Section 2.5 deals with the case $j<0$, and we prove that there is no non-zero function $\Psi_{j}$ of polynomial growth satisfying $\left[T_{f_{m, \delta}}, T_{\Psi_{j} e^{i j \theta}}\right]=0$ for $j<-2\left[\frac{\delta}{4}\right]$. By using similar techniques, Section 2.6 treats the case $0 \leq j \leq \delta$. In Section 2.7, some applications of our results are indicated. We show that the radial components $\Psi_{j}$ can be extended to a complex analytic function on the right half plane. Moreover, by our method we recover the examples in [17] of radial functions $u$ such that $T_{u}$ commutes with another Toeplitz operator having a non-radial symbol. Motivated by a conjecture of Louhichi and Rao in [129] and using our results, we give a triple $\left(T_{f}, T_{g}, T_{h}\right)$ of Toeplitz operators with $T_{f} \neq \mathrm{Id}$ such that $\left[T_{f}, T_{g}\right]=\left[T_{f}, T_{h}\right]=0$ but $\left[T_{g}, T_{h}\right] \neq 0$. Finally, we ask if for every $j \in \mathbb{Z}$ there is exactly one function $\Psi_{j}$ such that $\left[T_{f_{m, \delta}}, T_{\Psi_{j} e^{i j \theta}}\right]=0$.

\subsection{Preliminaries}

Let us start by defining the space of measurable functions of at most polynomialgrowth at infinity to be

$$
S:=\left\{g: \mathbb{C} \longrightarrow \mathbb{C} \mid \exists C, c>0 \text { s.t. }|g(z)| \leqslant C(1+|z|)^{c}\right\} .
$$

For a given $c>0$, we also define a function space:

$$
D_{c}:=\left\{\Psi: \mathbb{C} \longrightarrow \mathbb{C} \mid \Psi \text { measurable and } \exists d>0 \text { s.t. }|\Psi(z)| \leqslant d e^{c|z|^{2}}\right\}
$$

equipped with the norm $\|\Psi\|_{D_{c}}:=\left\|\Psi e^{-c|\cdot|^{2}}\right\|_{\infty}$. We write $\mathcal{F}_{c}:=\left(D_{c} \cap H^{2},\|\cdot\|_{D_{c}}\right)$ for the intersection with $H^{2}$ and we note that $\mathcal{E}:=\bigcup_{c<\frac{1}{4}} D_{c}$.

Throughout the chapter, $r$ and $\theta$ denote the polar coordinates of $z \in \mathbb{C}$ i.e. $z=r e^{i \theta}$. We fix a symbol $f_{m, \delta}\left(r e^{i \theta}\right)=r^{m} e^{i \delta \theta}$, where $m \in \mathbb{R}_{+}, \delta \in \mathbb{Z}$, and we write [.] for the greatest integer function. Note that each function $\Psi \in S$ can be expanded to an $L^{2}$-convergent series $\Psi\left(r e^{i \theta}\right)=\sum_{j=-\infty}^{\infty} \Psi_{j}(r) e^{i j \theta}$ (c.f. Lemma 2.2.1).

For a fixed symbol $f_{m, \delta}$ we aim to characterize $\Psi \in S$ such that the commutator $\left[T_{f_{m, \delta}}, T_{\Psi}\right]$ vanishes as an operator on the space of holomorphic polynomials $\mathbb{P}[z]$. It turns out that it is sufficient to characterize the coefficient functions $\left\{\Psi_{j}\right\}_{j \in \mathbb{Z}}$ in the series expansion above. We express $\left\{\Psi_{j}\right\}_{j \in \mathbb{Z}}$ in terms of the inverse Mellin transform of a function formed up of Gamma functions and a trigonometric polynomial. Moreover, if $f_{m, \delta}$ is a monomial (i.e. $m \pm \delta \in 2 \mathbb{N}_{0}$ ), then for each $j \in \mathbb{Z}$ such that $j \geqslant-2\left[\frac{|\delta|}{4}\right]$ we find a collection of quasi-homogeneous functions $\left\{\varphi_{j} e^{i j \theta}\right\} \subset \mathcal{E}$ such that $\left[T_{f_{m, \delta}}, T_{\varphi_{j} e^{\varepsilon i j \theta}}\right]=0$ on $\mathbb{P}[z]$ where $\varepsilon:=\operatorname{sign}(\delta)$.

First it is essential to make clear that the commutator $\left[T_{f_{m, \delta}}, T_{\Psi}\right]$ is well defined on $\mathbb{P}[z]$, and that it is sufficient to solve the above problem when $\delta \in \mathbb{N}_{0}$. 
Let $f \in S, \Psi \in \mathcal{E}$ and pick up $0<\epsilon<\frac{1}{4}$ such that $\Psi \in D_{\epsilon}$. We define a dense subspace $\mathcal{H}_{\epsilon} \subset H^{2}$ containing $\mathbb{P}[z]$ such that the operator products $T_{f} T_{\Psi}$ and $T_{\Psi} T_{f}$ are well defined on $\mathcal{H}_{\epsilon}$. Moreover, we prove the equivalence: $\left[T_{f}, T_{\Psi}\right]=0$ on $\mathcal{H}_{\epsilon}$ if and only if $\left[T_{\bar{f}}, T_{\bar{\Psi}}\right]=0$ on $\mathcal{H}_{\epsilon}$. In order to construct the space $\mathcal{H}_{\epsilon}$ we need to introduce a scale of subspaces (c.f. [14] ):

$$
D_{\epsilon} \subset D_{c_{1}} \subset D_{c_{2}} \subset \cdots \subset \bigcup_{j \in \mathbb{N}} D_{c_{j}} \subset L^{2},
$$

where $\left(c_{j}\right)_{j \in \mathbb{N}}$ is the increasing sequence of real numbers $c_{j}:=1 / 2-1 /(2 j+2)$ such that $c_{j} \in[1 / 4,1 / 2)$ for all $j \in \mathbb{N}$ and

$$
c_{j+1}=\frac{1}{4\left(1-c_{j}\right)} .
$$

It is known that the orthogonal projection $P$ is continuous from $D_{c_{j}}$ to $D_{c_{j+1}}$ for all $j \in \mathbb{N}$ (c.f. Lemma 10 in [14]). Using this fact, we can prove the following:

Proposition 2.2.1. Let $f \in S$ and fix $\epsilon<\frac{1}{4}$. Then there exists a densely embedded Hilbert space $\mathcal{H}_{\epsilon} \hookrightarrow H^{2}$ containing $\mathbb{P}[z]$ as well as the linear space

$$
\mathcal{L}=\operatorname{span}\left\{k_{z}(w):=K(w, z) e^{-\frac{1}{2}|z|^{2}}: z \in \mathbb{C}\right\}
$$

such that for all $\Psi \in D_{\epsilon} \subset \mathcal{E}$ the operator products $T_{\Psi} T_{f}$ and $T_{f} T_{\Psi}$ are well defined and continuous from $\mathcal{H}_{\epsilon}$ to $H^{2}$.

Proof. The first step in the proof is to find a positive number $\varsigma=\varsigma(\epsilon)$ such that the operator products $T_{\Psi} T_{f}$ and $T_{f} T_{\Psi}$ are well defined and continuous from $\mathcal{F}_{\varsigma}$ to $H^{2}$ for all $\Psi \in D_{\epsilon}$. Pick a real number $\gamma$ with $0<\gamma<1-\frac{1}{2(1-2 \epsilon)}$. Then there exists $j_{0} \in \mathbb{N}$ such that $\frac{1}{4(1-\gamma)}+\epsilon<c_{j_{0}}$, and one can find $j_{1} \geqslant j_{0}$ with $\varsigma:=c_{j_{1}+1}-c_{j_{1}}<\gamma$. Let $M_{f}$ and $M_{\Psi}$ denote the operators of multiplication by $f$ and $\Psi$, respectively. The assertion then follows by noting that $T_{\Psi} T_{f}$ is the composition of the following well defined and continuous operators:

$$
\mathcal{F}_{\varsigma} \stackrel{M_{f}}{\longrightarrow} D_{\gamma} \stackrel{P}{\rightarrow} \mathcal{F}_{\frac{1}{4(1-\gamma)}} \stackrel{M_{\Psi}}{\longrightarrow} D_{c_{j_{0}}} \stackrel{P}{\rightarrow} \mathcal{F}_{c_{j_{0}+1}} \hookrightarrow H^{2}
$$

Since $\varsigma+\epsilon<c_{j_{0}}+c_{j_{1}+1}-c_{j_{1}} \leqslant c_{j_{1}+1}$, the operator product $T_{f} T_{\Psi}$ is the composition of the continuous operators:

$$
\mathcal{F}_{\varsigma} \stackrel{M_{\Psi}}{\longrightarrow} D_{c_{j_{1}+1}} \stackrel{P}{\rightarrow} \mathcal{F}_{c_{j_{1}+2}} \stackrel{M_{f}}{\longrightarrow} D_{c_{j_{1}+3}} \stackrel{P}{\rightarrow} \mathcal{F}_{c_{j_{1}+4}} \hookrightarrow H^{2}
$$

Next, we modify $\mathcal{F}_{\varsigma}$ to obtain a densely embedded Hilbert space $\mathcal{H}_{\epsilon} \hookrightarrow \mathcal{F}_{\varsigma}$ such that $\mathcal{L} \cup \mathbb{P}[z] \subset$ $\mathcal{H}_{\epsilon}$ and the inclusion $\mathcal{L} \subset \mathcal{H}_{\epsilon}$ is dense. In particular, the restriction $\left[T_{f}, T_{\Psi}\right]: \mathcal{H}_{\epsilon} \longrightarrow H^{2}$ defines a continuous operator.

In order to construct the space $\mathcal{H}_{\epsilon}$ we fix a positive number $\lambda=\lambda(\epsilon)$ such that $\frac{\lambda}{2}<\varsigma$, and we denote by $\mu_{\lambda}$ the probability Gaussian measure on $\mathbb{C}$ given by

$$
d \mu_{\lambda}(z):=\frac{\lambda}{\pi} e^{-\lambda|z|^{2}} d v(z)
$$


Moreover, let $\langle\cdot, \cdot\rangle_{\lambda}$ be the usual inner product on $L^{2}\left(\mathbb{C}, d \mu_{\lambda}\right)$ and define

$$
\mathcal{H}_{\epsilon}:=H^{2}\left(\mathbb{C}, d \mu_{\lambda}\right)
$$

to be the subspace of all entire $\mu_{\lambda}$-square integrable functions on $\mathbb{C}$ equipped with the usual topology inherited from $L^{2}\left(\mathbb{C}, d \mu_{\lambda}\right)$ (with the notation used in Section 1.3, $\mathcal{H}_{\epsilon}$ is the SegalBargmann space $H_{\left(\frac{1}{4 \lambda}\right)}^{2}$ over $\left.\mathbb{C}\right)$. Then it is easy to check that $\mathcal{H}_{\epsilon}$ has the above mentioned properties.

Remark 2.2.1. In comparison to the case of the Bergman space over the unit disc considered in [66] and using the notation of Theorem AO the Toeplitz operators $T_{\psi}$ and $T_{\varphi}$ are bounded on $A(\mathbb{D})$. Hence $T_{\psi}$ and $T_{\varphi}$ commute on $A(\mathbb{D})$ if and only if they commute on the holomorphic polynomials defined on $\mathbb{D}$.

Remark 2.2.2. According to the above proposition, for a fixed $\epsilon<\frac{1}{4}$ the Toeplitz operator $T_{\Psi}$ is continuous on $\mathcal{H}_{\epsilon}$ for any $\Psi \in D_{\epsilon} \subset \mathcal{E}$. Since $\mathcal{L} \subset \mathcal{H}_{\epsilon}$, by Proposition 1.1.4 it follows that the Berezin transform is one-to-one on the space of Toeplitz operators with symbols in $D_{\epsilon}$ for any $\epsilon<\frac{1}{4}$. Moreover, since $D_{\epsilon_{1}} \subset D_{\epsilon_{2}}$ for $\epsilon_{1}<\epsilon_{2}$ and $\mathcal{E}=\bigcup_{\epsilon<\frac{1}{4}} D_{\epsilon}$ the Berezin transform is one-to-one on the space of Toeplitz operators with symbols in $\mathcal{E}$.

Another consequence of Proposition 1.1.4 we have:

Corollary 2.2.1. Let $f \in S$ and $\epsilon<\frac{1}{4}$ such that $\Psi \in D_{\epsilon} \subset \mathcal{E}$. Then $\left[T_{f}, T_{\Psi}\right]$ and $\left[T_{\bar{f}}, T_{\bar{\Psi}}\right]$ are well defined and continuous on $\mathcal{H}_{\epsilon}$ and satisfies

$$
\left[T_{f}, T_{\Psi}\right]=0 \Longleftrightarrow\left[T_{\bar{f}}, T_{\bar{\Psi}}\right]=0 .
$$

Proof. By a direct calculation, we obtain

$$
\widetilde{\left[T_{\bar{f}}, T_{\bar{\Psi}}\right]}=\overline{\left[\widetilde{T_{f}, T_{\Psi}}\right]}
$$

where $\widetilde{[\cdot, \cdot]}$ denotes the Berezin transform of the corresponding commutator. Since the operator $\left[T_{\bar{f}}, T_{\bar{\Psi}}\right]: \mathcal{H}_{\epsilon} \longrightarrow H^{2}$ is continuous, it follows from (2.2.1) and Proposition 1.1.4 that

$$
\left.\left[T_{f}, T_{\Psi}\right]=0 \Longleftrightarrow \widetilde{T_{f}, T_{\Psi}}\right]=0 \Longleftrightarrow\left[\widetilde{T_{\bar{f}}, T_{\bar{\Psi}}}\right]=0 \Longleftrightarrow\left[T_{\bar{f}}, T_{\bar{\Psi}}\right]=0 .
$$

The following $L^{2}$-series expansion is essential in our proofs:

Lemma 2.2.1. Let $\epsilon<\frac{1}{2}$ and $\Psi \in D_{\epsilon}$, then $\Psi$ has the series expansion:

$$
\Psi\left(r e^{i \theta}\right)=\sum_{j=-\infty}^{\infty} \Psi_{j}(r) e^{i j \theta}
$$

with $\left|\Psi_{j}(r)\right| \leqslant C e^{\epsilon r^{2}}$ for some constant $C>0$ and almost all $r>0$. The above convergence holds in $L^{2}(\mathbb{C}, d \mu)$. Moreover, if $\Psi \in S$ then $\Psi_{j}(r)$ is defined a.e. on $\mathbb{R}_{+}$and is of polynomial growth at infinity for all $j \in \mathbb{Z}$. 
Proof. If $\Psi \in S$, then by Lemma 4.2 in [17], $\Psi$ has an $L^{2}$-convergent expansion where each $\Psi_{j}(r)$ is of polynomial growth at infinity. Now, if $\Psi \in D_{\epsilon} \backslash S$ then is obtained from the expansion of the bounded function $\Psi(z) e^{-\epsilon|z|^{2}}$.

Remark 2.2.3. Let $\Psi \in \mathcal{E}$ and $f_{m, \delta}=r^{m} e^{i \delta \theta}$ be the function defined earlier. Assume that $\left[T_{f_{m, \delta}}, T_{\Psi}\right]=0$ on $\mathbb{P}[z]$. Then by Corollary 2.2.1 and due to the dense inclusion $\mathbb{P}[z] \subset \mathcal{H}_{\epsilon}$ it follows that $\left[T_{\overline{f_{m, \delta}}}, T_{\bar{\Psi}}\right]=\left[T_{f_{m,-\delta}}, T_{\bar{\Psi}}\right]=0$ on $\mathbb{P}[z]$. Since $(\bar{\Psi})_{j}=\Psi_{-j}$ for all $j \in \mathbb{Z}$, it is sufficient to consider only the case $\delta \in \mathbb{N}_{0}$ in the following.

Remark 2.2.4. In [66], it has been shown that any $\psi \in L^{2}(\mathbb{D}, d v)$ can be written as an $L^{2}\left(\mathbb{D}, \frac{1}{\pi} d v\right)$-series expansion $\psi\left(r e^{i \theta}\right)=\sum_{j=-\infty}^{\infty} \psi_{j}(r) e^{i j \theta}$. Indeed, if $\mathcal{R}$ denotes the space of complex valued functions defined on $[0,1)$ which are integrable with respect to the rdr measure $\left(r\right.$ varies in $[0,1)$ ) then the spaces $\left\{e^{i j \theta} \mathcal{R}\right\}_{j \in \mathbb{Z}}$ are mutually orthogonal in $L^{2}\left(\mathbb{D}, \frac{1}{\pi} d v\right)$. Moreover, every polynomial can be written as a finite sum in $\oplus_{j} e^{i j \theta} \mathcal{R}$. But the polynomials are dense in $L^{2}(\mathbb{D}, d v)$ hence $L^{2}(\mathbb{D}, d v)=\oplus_{j \in \mathbb{Z}} e^{i j \theta} \mathcal{R}$. As in the case of the Segal-Bargmann space considered in the above remark, Theorem AO treats also the case $\delta<0$. In fact, if $\psi$ and $\varphi\left(r e^{i \theta}\right)=r^{m} e^{i \delta \theta}$ are the bounded functions considered in Theorem AO then $T_{\varphi}$ and $T_{\psi}$ commute if and only if the adjoint operators $T_{\varphi}^{\star}=T_{r^{m} e^{-i \delta \theta}}$ and $T_{\psi}^{\star}=T_{\bar{\psi}}$ commute.

Now we recall some properties of the Mellin transform, which forms an important tool throughout this chapter. Let $g$ be a (suitable) complex valued function defined on the interval $(0, \infty)$. We write $M[g]$ for the Mellin transform of $g$ :

$$
M[g](z)=\int_{0}^{\infty} x^{z-1} g(x) d x .
$$

Recall that $M[g]$ is complex analytic on a strip in the complex plane parallel to the imaginary axis. For a suitable function $\varphi(z)$ which is complex analytic in a strip $a<\operatorname{Re}(z)<b$, the inverse Mellin transform $M^{-1}[\varphi]$ is the function on $(0, \infty)$ given by:

$$
M^{-1}[\varphi](x)=\frac{1}{2 \pi i} \int_{c-i \infty}^{c+i \infty} x^{-z} \varphi(z) d z
$$

where $c$ is any number between $a$ and $b$. Let $\Gamma(z)$ denote the Gamma function, and write

$$
H(x)= \begin{cases}0 & \text { if } x<0 \\ 1 & \text { if } x>0 \\ \frac{1}{2} & \text { if } x=0\end{cases}
$$

for the Heaviside step function. Then one has the following well known identities:

(1) $\operatorname{For} \operatorname{Re}(z)>0, \Gamma(z)=M\left[e^{-x}\right](z)$, and $\frac{1}{2} \Gamma\left(\frac{z}{2}\right)=M\left[e^{-x^{2}}\right](z)$.

(2) For $\operatorname{Re}(z)>0, \frac{1}{z}=M[H(1-x)](z)$. 
The relation (2) can be generalized as follows: Let $j \in \mathbb{Z}, n \in \mathbb{N}$ with $n \geqslant j$, then for $\operatorname{Re}(z)>-j$ we have:

$$
Q_{n}^{j}(x):=M^{-1}\left[\prod_{l=j}^{n}(z+l)^{-1}\right](x)=\frac{1}{(n-j) !} x^{j}(1-x)^{n-j} H(1-x) .
$$

Indeed, let $B(z, w)$ be the Beta function defined for $\operatorname{Re}(z), \operatorname{Re}(w)>0$ by:

$$
B(z, w):=\int_{0}^{1} x^{z-1}(1-x)^{w-1} d x=\frac{\Gamma(z) \Gamma(w)}{\Gamma(z+w)} .
$$

Note that

$$
\begin{aligned}
M\left[x^{j}(1-x)^{n-j} H(1-x)\right](z) & =\int_{0}^{1} x^{z+j-1}(1-x)^{n-j} d x=B(z+j, n-j+1) \\
& =\frac{\Gamma(z+j) \Gamma(n-j+1)}{\Gamma(z+n+1)} \\
& =\frac{\Gamma(z) \Gamma(n-j+1) \prod_{l=0}^{j-1}(z+l)}{\Gamma(z) \prod_{l=0}^{n}(z+l)} \\
& =\frac{(n-j) !}{\prod_{l=j}^{n}(z+l)}
\end{aligned}
$$

We shortly write $Q_{n}(x):=Q_{n}^{1}(x)$. If $\phi \in D_{c}$ is a radial function on $\mathbb{C}$, i.e. $\phi(z)=\phi(|z|)=$ $\phi(r)$, with $c<1$ then $M\left[\phi(r) e^{-r^{2}}\right](z)$ exists for all $z \in \mathbb{C}$ such that $\operatorname{Re}(z)>0$.

In order to relate the Mellin transform of a function to Toeplitz operators, we need the following simple observation:

Proposition 2.2.2. Let $\phi \in \mathcal{E}$ be a radial function on $\mathbb{C}$, and let $k \in \mathbb{N}_{0}$. Then:

(1) For $l \in \mathbb{N}_{0}$, we have:

$$
P\left(\phi r^{k} e^{i l \theta}\right)=2 M\left[\phi e^{-r^{2}}\right](l+k+2) \frac{z^{l}}{l !}
$$

(2) For $l \in\{\cdots,-2,-1\}$, it holds $P\left(\phi r^{k} e^{i l \theta}\right)=0$.

Proof. (1) : For $l \in \mathbb{N}_{0}$

$$
\begin{aligned}
P\left(\phi r^{k} e^{i l \theta}\right) & =\left\langle\phi r^{k} e^{i l \theta}, K(\cdot, z)\right\rangle \\
& =\sum_{n \geqslant 0}\left\langle\phi r^{k} e^{i l \theta}, \frac{\bar{z}^{n} r^{n} e^{i n \theta}}{n !}\right\rangle \\
& =\frac{1}{\pi} \sum_{n \geqslant 0} \int_{0}^{2 \pi} e^{i(l-n) \theta} d \theta \int_{0}^{\infty} r^{k+n+1} \phi(r) e^{-r^{2}} d r \frac{z^{n}}{n !} \\
& =2 M\left[\phi e^{-r^{2}}\right](l+k+2) \frac{z^{l}}{l !} .
\end{aligned}
$$

(2) : The second assertion follows by a similar calculation. 


\subsection{Necessary and sufficient conditions for the commuting problem}

For each $\mu \in \mathbb{N}_{0}$, we define $S_{\mu} \subset S$ to be the subspace of quasi-homogeneous symbols of degree $\mu$ :

$$
S_{\mu}:=\left\{f \in \mathbb{C} \longrightarrow \mathbb{C} \mid f\left(r e^{i \theta}\right)=g(r) e^{i \mu \theta} \text { for some radial function } g \in S\right\} .
$$

Note that, by an application of the previous proposition, for any $f \in S_{\mu}$ we have $T_{f} z^{k} \in S_{\mu+k} \subset$ $S$ for all $k \in \mathbb{N}_{0}$.

Remark 2.3.1. In general, and even in the case of a bounded symbol $g$ the function $T_{g} z^{k}$ needs not to be in $S$. In fact, if we put $g(z):=e^{i \operatorname{Re}(z)}$, then

$$
[P g](z)=\int g(w) e^{\frac{i}{2}(w+\bar{w})} e^{z \bar{w}} d \mu(w)=\int g(w) e^{\frac{i}{2} w} e^{\bar{w}\left(\frac{i}{2}+z\right)} d \mu(w)=\left[P e^{\frac{i}{2}(\cdot)}\right]\left(\frac{i}{2}+z\right)=e^{\frac{-1}{4}+\frac{i}{2} z}
$$

Proposition 2.3.1. Let $f \in S_{\mu}$, and fix $\Psi \in \mathcal{E}$ having the expansion Then (1), (2) and (3) below are equivalent:

(1) $T_{f} T_{\Psi}=T_{\Psi} T_{f}$

(2) $T_{f} T_{\Psi_{j} e^{i j \theta}}=T_{\Psi_{j} e^{i j \theta}} T_{f}, \quad$ for all $j \in \mathbb{Z}$.

(3) For each $j \in \mathbb{Z}$, the relations (a) and (b) hold:

(a) For all $k \in \mathbb{N}_{0}$ such that $k \geqslant-j$ :

$$
\begin{aligned}
& M\left[g e^{-r^{2}}\right](2 k+\mu+2) \cdot M\left[\Psi_{j} e^{-r^{2}}\right](2 k+2 \mu+j+2) \frac{(j+k) !}{(\mu+k) !} \\
& =M\left[g e^{-r^{2}}\right](2 k+2 j+\mu+2) \cdot M\left[\Psi_{j} e^{-r^{2}}\right](2 k+j+2) .
\end{aligned}
$$

(b) For all $k \in \mathbb{N}_{0}$ such that $-\mu-j \leqslant k \leqslant-j-1$ :

$$
M\left[g e^{-r^{2}}\right](2 k+\mu+2) \cdot M\left[\Psi_{j} e^{-r^{2}}\right](2 k+2 \mu+j+2)=0 .
$$

Proof. For each $k \in \mathbb{N}_{0}$, and using the previous proposition we know that:

$$
T_{\Psi_{j} e^{i j \theta}} z^{k}=P\left(\Psi_{j} r^{k} e^{i(j+k) \theta}\right)= \begin{cases}2 M\left[\Psi_{j} e^{-r^{2}}\right](2 k+j+2) \frac{z^{j+k}}{(j+k) !} & \text { if } k \geqslant-j ; \\ 0 & \text { if } k<-j .\end{cases}
$$

Since $f=g e^{i \mu \theta} \in S_{\mu}$ and $\mu \in \mathbb{N}_{0}$ we have:

$$
T_{f} z^{k}=P\left(g e^{i(\mu+k) \theta} r^{k}\right)=2 M\left[g e^{-r^{2}}\right](2 k+\mu+2) \frac{z^{\mu+k}}{(\mu+k) !} .
$$


Combining these relations gives:

$$
\begin{aligned}
& T_{f} T_{\Psi_{j} e^{i j \theta}} z^{k}=c_{1}(j, k) z^{\mu+j+k} \\
& = \begin{cases}4 M\left[\Psi_{j} e^{-r^{2}}\right](2 k+j+2) \frac{1}{(j+k) !} M\left[g e^{-r^{2}}\right](2 k+2 j+\mu+2) \frac{z^{\mu+j+k}}{(\mu+j+k) !} & \text { if } k \geqslant-j \\
0 & \text { if } k<-j,\end{cases}
\end{aligned}
$$

and:

$$
\begin{aligned}
& T_{\Psi_{j} e^{i j \theta} T_{f} z^{k}=c_{2}(j, k) z^{\mu+j+k}} \\
& = \begin{cases}4 M\left[\Psi_{j} e^{-r^{2}}\right](2 k+2 \mu+j+2) \frac{1}{(\mu+k) !} M\left[g e^{-r^{2}}\right](2 k+\mu+2) & \frac{z^{\mu+j+k}}{(\mu+j+k) !} \\
0 & \text { if } k \geqslant-\mu-j \\
0 & \text { if } k<-\mu-j\end{cases}
\end{aligned}
$$

Note that, $T_{f} T_{\Psi_{j} e^{i j \theta}} z^{k}=T_{\Psi_{j} e^{i j \theta}} T_{f} z^{k} \equiv 0$ for all $k$ such that $k<-\mu-j$.

The next step now, is to prove the uniformly compact convergence on $\mathbb{C}$ of:

(i) $\sum_{j=-\infty}^{\infty} T_{\Psi_{j} e^{i j \theta}} z^{k}=T_{\Psi} z^{k}$,

(ii) $\sum_{j=-\infty}^{\infty} T_{f} T_{\Psi_{j} e^{i j \theta}} z^{k}=T_{f} T_{\Psi} z^{k}$

(iii) $\sum_{j=-\infty}^{\infty} T_{\Psi_{j} e^{i j \theta}} T_{f} z^{k}=T_{\Psi} T_{f} z^{k}$

which follows from Lemma 2 and the following estimates (we only prove (i) and (ii)). Let $N \in \mathbb{N}$ then

(i):

$$
\begin{aligned}
\left|P\left(\sum_{j=-N}^{N} \Psi_{j}(r) e^{i j \theta} z^{k}-\Psi z^{k}\right)(z)\right| & =\left|\left\langle\sum_{j=-N}^{N} \Psi_{j}(r) e^{i j \theta} w^{k}-\Psi(w) w^{k}, e^{\bar{z} w}\right\rangle\right| \\
& \leqslant\left\|\sum_{j=-N}^{N} \Psi_{j}(r) e^{i j \theta}-\Psi(w)\right\|\left\|w^{k} e^{z \bar{w}}\right\| .
\end{aligned}
$$

(ii):

$$
\begin{gathered}
\left|P\left(f P\left(\sum_{j=-N}^{N} \Psi_{j}(r) e^{i j \theta} z^{k}\right)-f P\left(\Psi z^{k}\right)\right)(z)\right|= \\
\mid\left\langle P\left(\sum_{j=-N}^{N} \Psi_{j}(r) e^{i j \theta} w^{k}-\Psi(w) w^{k}, \bar{f} e^{\bar{z} w}\right\rangle\right| \leqslant\left\|\sum_{j=-N}^{N} \Psi_{j}(r) e^{i j \theta}-\Psi(w)\right\|\left\|f w^{k} e^{z \bar{w}}\right\| .
\end{gathered}
$$

Therefore:

$$
T_{f} T_{\Psi} z^{k}=\sum_{j=-\infty}^{\infty} T_{f} T_{\Psi_{j} e^{i j \theta}} z^{k}=\sum_{j=-k}^{\infty} c_{1}(j, k) z^{\mu+j+k}
$$


and

$$
T_{\Psi} T_{f} z^{k}=\sum_{j=-\infty}^{\infty} T_{\Psi_{j} e^{i j \theta}} T_{f} z^{k}=\sum_{j=-\mu-k}^{\infty} c_{2}(j, k) z^{\mu+j+k} .
$$

The proposition then follows from the identity of the two power series and the last two conditions.

Now, we apply the above proposition to $f=f_{m, \delta}$ (as we remarked before, we can assume that $\delta \geqslant 0$ ) to obtain the following theorem:

Theorem 2.3.1. Let $\Psi \in \mathcal{E}$, then the following are equivalent:

(1) $T_{f_{m, \delta}} T_{\Psi}=T_{\Psi} T_{f_{m, \delta}}$.

(2) $T_{f_{m, \delta}} T_{\Psi_{j} e^{i j \theta}}=T_{\Psi_{j} e^{i j \theta}} T_{f_{m, \delta}}$ for all $j \in \mathbb{Z}$.

(3) For each $j \in \mathbb{Z}$, we have:

(a) For every $k \in \mathbb{N}_{0}$ with $k \geqslant-j$ the following equation holds

$$
\begin{array}{ll}
\frac{\Gamma\left(k+1+\frac{\delta+m}{2}\right)}{\Gamma\left(k+j+1+\frac{\delta+m}{2}\right)} M\left[\Psi_{j} e^{-r^{2}}\right](2 k+2 \delta+j+2)= \\
\frac{\Gamma(k+\delta+1)}{\Gamma(k+j+1)} M\left[\Psi_{j} e^{-r^{2}}\right](2 k+j+2) .
\end{array}
$$

(b) For each $k \in \mathbb{N}_{0}$ such that $-\delta-j \leqslant k \leqslant-j-1$, we have

$$
M\left[\Psi_{j} e^{-r^{2}}\right](2 k+2 \delta+j+2)=0
$$

Proof. The equivalence of (1), (2) and (3) directly follows from an application of the previous proposition and the fact that:

1. $M\left[r^{m} e^{-r^{2}}\right](2 k+\delta+2)=\frac{1}{2} \Gamma\left(k+1+\frac{\delta+m}{2}\right) \neq 0$.

2. $M\left[r^{m} e^{-r^{2}}\right](2 k+2 j+\delta+2)=\frac{1}{2} \Gamma\left(k+j+1+\frac{\delta+m}{2}\right) \neq 0$.

Remark 2.3.2. For the case considered in Theorem AO and by Remark 2.2.1 the Toeplitz operators $T_{\varphi}$ and $T_{\psi}$ commute on $A(\mathbb{D})$ if and only if for each $k \in \mathbb{N}_{0}$ we have $T_{\varphi} T_{\psi} z^{k}=T_{\psi} T_{\varphi} z^{k}$. By direct calculation (c.f. p. 201 in [66]) and similar to the above theorem, this is equivalent to say that for each fixed $j \in \mathbb{Z}$ and for every $k \in \mathbb{N}_{0}$ such that $k \geq-j$ the following equation holds

$$
M\left[\psi_{j}\right](2 k+2 \delta+j+2)=M\left[\psi_{j}\right](2 k+j+2) \frac{(2 k+2 j+2)(2 k+\delta+m+2)}{(2 k+2 \delta+2)(2 k+2 j+\delta+m+2)} .
$$


Analogous to condition (2) in Theorem 2.3.1, Equation (2.3.3) shows that $T_{\varphi} T_{\psi}=T_{\psi} T_{\varphi}$ if and only if for each $j \in \mathbb{Z}$ we have $T_{\varphi} T_{\psi_{j}(r) e^{i j \theta}}=T_{\psi_{j}(r) e^{i j \theta}} T_{\varphi}$. Moreover, since $\psi_{j}$ is supported in $[0,1]$ and bounded (since $\psi$ is bounded) it follows that $M\left[\psi_{j}\right](z)$ is bounded as function of $z$ on the right half-plane $\operatorname{Re}(z) \geq 1$. This shows that the function

$$
M\left[\psi_{j}\right](z+2 \delta)-M\left[\psi_{j}\right](z) \frac{(z+j)(z-j+\delta+m)}{(z-j+2 \delta)(z+j+\delta+m)}
$$

is bounded, analytic and vanishing at the integers in some right half-plane. However by the Blaschke condition a bounded analytic function can not have zeros that form an arithmetic sequence unless it is the zero function. Therefore the Toeplitz operators $T_{\varphi}$ and $T_{\psi}$ commute on $A(\mathbb{D})$ if and only iffor each $j \in \mathbb{Z}$ the following equation

$$
M\left[\psi_{j}\right](z+2 \delta)=M\left[\psi_{j}\right](z) \frac{(z+j)(z-j+\delta+m)}{(z-j+2 \delta)(z+j+\delta+m)}
$$

holds true on some right half-plane.

Now suppose that $\Psi \in S$ and we fix $j \in \mathbb{Z}$. Similar to the above remark, we aim to prove that the relation (2.3.1) extends from all $z=k \in \mathbb{N}_{0}$ to all $z \in \mathbb{C}$ such that $\operatorname{Re}(z)>-j-1$. For this purpose, we need to define the following space of functions:

$$
\begin{array}{r}
\mathcal{A}=\left\{u: \mathbb{R}^{+} \longrightarrow \mathbb{C} \mid \exists \quad C, c, p, \gamma>0 \text { s.t. }|u(x)| \leqslant \frac{C}{x^{p}} \text { for } x \in(0,1]\right. \\
\text { and } \left.|u(x)| \leqslant c x^{\gamma} \text { for } x \in[1, \infty)\right\} .
\end{array}
$$

The following two lemmas are found in [17]:

Lemma 2.3.1. For $u, v \in \mathcal{A}$, set $f_{u}(x):=u(x) e^{-x^{2}}$ and $f_{v}(x):=v(x) e^{-x^{2}}$. Then the Mellin convolution product $f_{u} * f_{v}$ exists on $\mathbb{R}_{+}$, and there is $h \in \mathcal{A}$ such that:

$$
\left(f_{u} * f_{v}\right)(x)=h(x) e^{-x} .
$$

The following lemma replaces the Blaschke condition in the unit disc (c.f. Remark 2.3.2).

Lemma 2.3.2. Let $u \in \mathcal{A}$, and fix a number $a \in(0,2]$. Suppose there is $m_{0} \in \mathbb{N}_{0}$ with the property:

$$
M\left[u e^{-x}\right](a k+1)=\int_{0}^{\infty} u(x) e^{-x} x^{a k} d x=0
$$

for all $k \geqslant m_{0}$. Then $u=0$ a.e. on $\mathbb{R}^{+}$.

In order to determine the components $\Psi_{j}$ of $\Psi$ in the expansion whenever $\left[T_{f_{m, \delta}}, T_{\Psi}\right] \equiv 0$, we need to distinguish three cases:

- Case $j>\delta$.

- Case $j<0$.

- Case $0 \leqslant j \leqslant \delta$.

A detailed analysis is done in the following three sections. 


\subsection{Case $j>\delta$}

Since $j>0$ and $\delta \geqslant 0$ the case $-\delta-j \leqslant k \leqslant-j-1$ in Theorem 2.3.1, (3)(b) does not occur for any $k \in \mathbb{N}_{0}$ and hence $T_{f_{m, \delta}} T_{\Psi_{j} e^{i j \theta}}=T_{\Psi_{j} e^{i j \theta}} T_{f_{m, \delta}}$ holds if and only if the Equation (2.3.1) is fulfilled for all $k \in \mathbb{N}_{0}$.

Proposition 2.4.1. Let $\Psi \in S$ and $j>\delta$, then $T_{f_{m, \delta}} T_{\Psi_{j} e^{i j \theta}}=T_{\Psi_{j} e^{i j \theta}} T_{f_{m, \delta}}$ holds if and only if the following functional equation:

$$
M\left[\Psi_{j} e^{-r^{2}}\right](2 z+2 \delta+j+2)=M\left[\Psi_{j} e^{-r^{2}}\right](2 z+j+2) \prod_{l=1}^{j}\left(z+\frac{\delta+m}{2}+l\right) \prod_{l=1}^{j-\delta}(z+\delta+l)^{-1}
$$

is fulfilled in the half plane $\operatorname{Re}(z)>-\frac{j}{2}-1$.

Proof. First we note that $M\left[\Psi_{j} e^{-r^{2}}\right](2 z+j+2)$ exists for $\operatorname{Re}(z)>-\frac{j}{2}-1$. Now, by the functional equation of the Gamma function one obtains for each $j>\delta$ and for $\operatorname{Re}(z)>-j-1$ :

$$
\begin{aligned}
& \text { - } \frac{\Gamma(z+\delta+1)}{\Gamma(z+j+1)}=\prod_{l=1}^{j-\delta}(z+\delta+l)^{-1}, \\
& \text { - } \frac{\Gamma\left(z+\frac{\delta+m}{2}+1\right)}{\Gamma\left(z+\frac{\delta+m}{2}+j+1\right)}=\prod_{l=1}^{j}\left(z+\frac{\delta+m}{2}+l\right)^{-1}
\end{aligned}
$$

and (2.3.1) takes the form:

$$
M\left[\Psi_{j} e^{-r^{2}}\right](2 k+2 \delta+j+2) \prod_{l=1}^{j}\left(k+\frac{\delta+m}{2}+l\right)^{-1}=M\left[\Psi_{j} e^{-r^{2}}\right](2 k+j+2) \prod_{l=1}^{j-\delta}(k+\delta+l)^{-1} .
$$

Recall that the Mellin transform of a function $g$ satisfies the transformation rule:

$$
M\left[g\left(x^{a}\right)\right](z)=\frac{1}{a} M[g]\left(\frac{z}{a}\right),
$$

for all $a>0$ such that $M[g]$ is defined for $\frac{z}{a}$. Together with (2.2.2) we get

$$
\prod_{l=1}^{j-\delta}(z+\delta+l)^{-1}=M\left[r^{\delta} Q_{j-\delta}(r)\right](z)=2 M\left[r^{2 \delta} Q_{j-\delta}\left(r^{2}\right)\right](2 z)
$$

and

$$
\prod_{l=1}^{j}\left(z+\frac{\delta+m}{2}+l\right)^{-1}=M\left[r^{\frac{\delta+m}{2}} Q_{j}(r)\right](z)=2 M\left[r^{\delta+m} Q_{j}\left(r^{2}\right)\right](2 z) .
$$

By substituting these quantities into (2.4.2) we obtain for all $k \in \mathbb{N}_{0}$ and for all $j>\delta$ :

$$
M\left[r^{j+2 \delta+2} \Psi_{j} e^{-r^{2}}\right](2 k) \cdot M\left[r^{\delta+m} Q_{j}\left(r^{2}\right)\right](2 k)=M\left[r^{j+2} \Psi_{j} e^{-r^{2}}\right](2 k) \cdot M\left[r^{2 \delta} Q_{j-\delta}\left(r^{2}\right)\right](2 k) .
$$

Now consider

$$
v_{j}(r):=r^{\delta+m} Q_{j}\left(r^{2}\right) e^{r^{2}}, \text { and } u_{j}(r):=r^{j+2 \delta+2} \Psi_{j}(r) .
$$


Since $Q_{j}$ is supported in $[0,1]$ and $\Psi_{j} \in \mathcal{A}$ we conclude that $u_{j}, v_{j} \in \mathcal{A}$. Therefore, by Lemma 2.3.1 the convolution product $\left(f_{u_{j}} * f_{v_{j}}\right)(r)$ exists for all $r>0$, and there is $h_{j} \in \mathcal{A}$ such that

$$
\left(r^{\delta+m} Q_{j}\left(r^{2}\right)\right) *\left(r^{j+2 \delta+2} \Psi_{j}(r) e^{-r^{2}}\right)(r)=h_{j}(r) e^{-r} .
$$

The same argument can be used on the right-hand side of (2.4.3) to show that there is $n_{j} \in \mathcal{A}$ such that

$$
\left(r^{j+2} \Psi_{j}(r) e^{-r^{2}}\right) *\left(r^{2 \delta} Q_{j-\delta}\left(r^{2}\right)\right)(r)=n_{j}(r) e^{-r} .
$$

By substituting these relations into (2.4.3) and applying the convolution theorem for the Mellin transform, we obtain for any $k \in \mathbb{N}_{0}$ :

$$
M\left[\left(h_{j}-n_{j}\right) e^{-r}\right](2 k)=M\left[\frac{h_{j}-n_{j}}{r} e^{-r}\right](2 k+1)=0 .
$$

According to Lemma 2.3.2 we have $h_{j}=n_{j}$ a.e. on $\mathbb{R}_{+}$, i.e $M\left[\left(h_{j}-n_{j}\right) e^{-r}\right](2 z)=0$, so that for $\operatorname{Re}(z)>-\frac{j}{2}-1$ the functional equation (2.4.1) holds.

Example 2.4.1. (c.f. [17]) Put $\delta=0$ and consider the symbol $f(z)=f_{m, 0}=r^{m}$ with $m \in \mathbb{R}_{+}$. Assume that $\Psi \in S$ with $\left[T_{f}, T_{\Psi}\right] \equiv 0$ on $\mathbb{P}[z]$. Then by Theorem 2.3.1, for each $j \in \mathbb{N}$ and for every $k \in \mathbb{N}_{0}$ we have

$$
\frac{\Gamma\left(k+1+\frac{m}{2}\right)}{\Gamma\left(k+j+1+\frac{m}{2}\right)} M\left[\Psi_{j} e^{-r^{2}}\right](2 k+j+2)=\frac{\Gamma(k+1)}{\Gamma(k+j+1)} M\left[\Psi_{j} e^{-r^{2}}\right](2 k+j+2) .
$$

Let $m \neq 0$, then we want to prove that $\Psi_{j}=0$ for all $j \neq 0$. For this, suppose there is $j>0$, such that $\Psi_{j} \neq 0$. Then by Lemma 2.3.2 there is $k_{0} \in \mathbb{N}_{0}$ with

$$
M\left[\Psi_{j} e^{-r^{2}}\right]\left(2 k_{0}+j+2\right) \neq 0 .
$$

According to (2.4.4) we get

$$
\frac{\Gamma\left(k_{0}+\frac{m}{2}+1\right)}{\Gamma\left(k_{0}+j+\frac{m}{2}+1\right)}=\frac{\Gamma\left(k_{0}+1\right)}{\Gamma\left(k_{0}+j+1\right)}
$$

and therefore

$$
\prod_{l=1}^{j}\left(k_{0}+\frac{m}{2}+l\right)=\prod_{l=1}^{j}\left(k_{0}+l\right)
$$

which contradicts our assumption $m \neq 0$. Hence, for $m \neq 0$ we have $\Psi_{j}=0$ for all $j>0$. By Corollary 2.2.1 it holds $T_{\bar{\Psi}} T_{f}=T_{f} T_{\bar{\Psi}}$, and the same argument shows that $\Psi_{j}=0$ for all $j<0$, i.e. $\Psi=\Psi_{0}$ is radial.

In this part of the chapter, we aim to find functions $\left\{\Psi_{j}\right\}_{j>\delta}$ such that $M\left[\Psi_{j} e^{-r^{2}}\right]$ satisfy the functional equation (2.4.1). For this purpose, and for each $j>\delta$ we define the meromorphic function:

$$
G_{j}(z):=\prod_{l=1}^{j} \Gamma\left(\frac{z+p+l}{\delta}\right) \prod_{l=1}^{j-\delta}\left[\Gamma\left(\frac{z+\delta+l}{\delta}\right)\right]^{-1} \delta^{z}
$$

where $p:=\frac{\delta+m}{2} \in \mathbb{R}_{+}$. 
Proposition 2.4.2. Let $\Psi \in S$ and suppose that $T_{f_{m, \delta}} T_{\Psi}=T_{\Psi} T_{f_{m, \delta}}$. Then for each $j>\delta$ :

$$
\mathcal{F}_{j}(z):=\frac{M\left[\Psi_{j} e^{-r^{2}}\right](2 z+j+2)}{G_{j}(z)}
$$

defines a $\delta$-periodic entire function.

Proof. Recall that $G_{j}(z)$ is defined on $\left\{z \in \mathbb{C} \mid z+p+l \neq-k \delta, l=1, \cdots, j\right.$ and $\left.k \in \mathbb{N}_{0}\right\}$. In the case $\operatorname{Re}(z)$ sufficiently large, and by using the functional equation of the Gamma function we have:

$$
\begin{aligned}
G_{j}(z+\delta) & =\prod_{l=1}^{j} \Gamma\left(\frac{z+p+l}{\delta}+1\right) \prod_{l=1}^{j-\delta}\left[\Gamma\left(\frac{z+\delta+l}{\delta}+1\right)\right]^{-1} \delta^{z+\delta} \\
& =\prod_{l=1}^{j}\left[\frac{z+p+l}{\delta} \Gamma\left(\frac{z+p+l}{\delta}\right)\right] \prod_{l=1}^{j-\delta}\left[\frac{z+\delta+l}{\delta} \Gamma\left(\frac{z+\delta+l}{\delta}\right)\right]^{-1} \delta^{z+\delta} \\
& =G_{j}(z) \prod_{l=1}^{j}(z+p+l) \prod_{l=1}^{j-\delta}(z+\delta+l)^{-1} .
\end{aligned}
$$

Since by Theorem 2.3.1 and Proposition 2.4.1 the Mellin transform $M\left[\Psi_{j} e^{-r^{2}}\right](z)$ is a solution of the functional equation (2.4.1) we obtain

$$
\begin{aligned}
\mathcal{F}_{j}(z+\delta) & =\frac{M\left[\Psi_{j} e^{-r^{2}}\right](2 z+2 \delta+j+2)}{G_{j}(z+\delta)} \\
& =\frac{M\left[\Psi_{j} e^{-r^{2}}\right](2 z+2 \delta+j+2)}{G_{j}(z)} \prod_{l=1}^{j}(z+p+l)^{-1} \prod_{l=1}^{j-\delta}(z+\delta+l) \\
& =\frac{M\left[\Psi_{j} e^{-r^{2}}\right](2 z+j+2)}{G_{j}(z)}=\mathcal{F}_{j}(z) .
\end{aligned}
$$

Therefore, $\mathcal{F}_{j}(z)$ is a $\delta$-periodic holomorphic function in some right half plane. Hence it can be extended to an entire function on the complex plane.

In the rest of this section, we assume that $\Psi \in S$ and $T_{f_{m, \delta}} T_{\Psi}=T_{\Psi} T_{f_{m, \delta}}$. Now, we want to prove that for each $j>\delta$, the entire function $\mathcal{F}_{j}(z)$ is a trigonometric polynomial. For this we need Stirling's formula together with the following well known result (c.f. [132]):

Lemma 2.4.1. Let $g$ be an entire function on $\mathbb{C}$ with period $\delta \in \mathbb{N}$. Suppose, there are $A, B>0$ such that $|g(z)| \leqslant A e^{B|z|}$. Then $g$ is a trigonometric polynomial of the form:

$$
g(z)=\sum_{l=-n}^{n} a_{l} e^{\frac{2 \pi i l z}{\delta}}, \quad a_{l} \in \mathbb{C}, \quad n \in \mathbb{N} .
$$

Applying Lemma 2.4.1 to the periodic function $\mathcal{F}_{j}(z)$, we can prove:

Proposition 2.4.3. For each $j>\delta$, there exists a trigonometric polynomial $\sum_{|l|<\frac{\delta}{4}} a_{l} e^{\frac{2 \pi i l z}{\delta}} s u c h$ that

$$
M\left[\Psi_{j} e^{-r^{2}}\right](2 z+j+2)=G_{j}(z) \sum_{|l|<\frac{\delta}{4}} a_{l} e^{\frac{2 \pi i l z}{\delta}} .
$$


Proof. For $\operatorname{Re}(z)>0$, it is well known that the Gamma function has the following asymptotic behavior as $|z| \rightarrow \infty$, (c.f. [175]):

$$
\Gamma(z)=e^{\left(z-\frac{1}{2}\right) \log (z)-z+\frac{\log (2 \pi)}{2}} \times\left\{1+o\left(\frac{1}{z}\right)\right\} .
$$

It follows that

$$
\prod_{l=1}^{j} \Gamma\left(\frac{z+p+l}{\delta}\right)=\prod_{l=1}^{j} e^{\left(\frac{z+p+l}{\delta}-\frac{1}{2}\right) \log \left(\frac{z+p+l}{\delta}\right)-\frac{z+p+l}{\delta}+\frac{\log (2 \pi)}{2}} \times\left\{1+o\left(\frac{1}{z}\right)\right\} .
$$

Using

$$
\log \left(\frac{z+p+l}{\delta}\right)=\log \left(\frac{z}{\delta}\right)+\log \left(1+\frac{p+l}{z}\right),
$$

the exponent can be decomposed in the form:

$$
\begin{aligned}
\left(\frac{z+p+l}{\delta}-\frac{1}{2}\right) \log \left(\frac{z+p+l}{\delta}\right)= & \left(\frac{z+p+l}{\delta}-\frac{1}{2}\right) \log \left(\frac{z}{\delta}\right) \\
& +\left(\frac{z+p+l}{\delta}-\frac{1}{2}\right) \log \left(1+\frac{p+l}{z}\right) \\
= & \left(\frac{z+p+l}{\delta}-\frac{1}{2}\right) \log \left(\frac{z}{\delta}\right)+O(1), \quad|z| \rightarrow \infty .
\end{aligned}
$$

We insert this relation into (2.4.6):

$$
\begin{aligned}
\prod_{l=1}^{j} \Gamma\left(\frac{z+p+l}{\delta}\right)=\exp & \left\{\sum_{l=1}^{j}\left[\left(\frac{z+p+l}{\delta}-\frac{1}{2}\right) \log \left(\frac{z}{\delta}\right)+O(1)-\frac{z+p+l}{\delta}\right]\right\} \\
& \times\left\{1+o\left(\frac{1}{z}\right)\right\} \\
=\exp & \left\{\left[\left(\frac{z+p}{\delta}-\frac{1}{2}\right) \log \left(\frac{z}{\delta}\right)-\frac{z}{\delta}\right] j+\frac{j(j+1)}{2 \delta} \log \left(\frac{z}{\delta}\right)+O(1)\right\} \\
& \times\left\{1+o\left(\frac{1}{z}\right)\right\} .
\end{aligned}
$$

Similarly, one has as $|z| \rightarrow \infty$

$$
\begin{aligned}
\prod_{l=1}^{j-\delta}\left[\Gamma\left(\frac{z+\delta+l}{\delta}\right)\right]^{-1}=\exp & \left\{\sum_{l=1}^{j-\delta}\left[\left(\frac{1}{2}-\frac{z+\delta+l}{\delta}\right) \log \left(\frac{z}{\delta}\right)+O(1)+\frac{z+\delta+l}{\delta}\right]\right\} \\
\times & \left\{1+o\left(\frac{1}{z}\right)\right\} \\
=\exp \{ & {\left[\frac{z}{\delta} \log \left(\frac{z}{\delta}\right)+\frac{1}{2} \log \left(\frac{z}{\delta}\right)-\frac{z}{\delta}\right](\delta-j) } \\
& \left.\quad-\frac{(j-\delta)(j-\delta+1)}{2 \delta} \log \left(\frac{z}{\delta}\right)+O(1)\right\} \times\left\{1+o\left(\frac{1}{z}\right)\right\} .
\end{aligned}
$$


Hence

$$
\begin{aligned}
\prod_{l=1}^{j} \Gamma\left(\frac{z+p+l}{\delta}\right) & \prod_{l=1}^{j-\delta}\left[\Gamma\left(\frac{z+\delta+l}{\delta}\right)\right]^{-1} \\
& =\exp \left\{z \log \left(\frac{z}{\delta}\right)+\left(\frac{p j}{\delta}+\frac{1}{2}\right) \log \left(\frac{z}{\delta}\right)-z+O(1)\right\}\left\{1+o\left(\frac{1}{z}\right)\right\} .
\end{aligned}
$$

We choose a branch of $\log (z)$ such that $\log \left(\delta^{z}\right)=z \log (\delta)$. Then we can write

$$
G_{j}(z)=\exp \left\{z \log (z)+s \log \left(\frac{z}{\delta}\right)-z+O(1)\right\} \times\left\{1+o\left(\frac{1}{z}\right)\right\},
$$

where $s:=\left(\frac{p j}{\delta}+\frac{1}{2}\right)$. If we write $z=\sigma+i t$, then we obtain the following asymptotic behavior of $\left|G_{j}(z)\right|$ as $|z| \longrightarrow \infty$ :

$$
\left|G_{j}(z)\right|=\exp \{(\sigma+s) \log (|z|)-\sigma-t \cdot \arg (z)+O(1)\} \times\left\{1+o\left(\frac{1}{|z|}\right)\right\} .
$$

Since $\Psi_{j} \in \mathcal{A}$, there is some $c>0$ such that

$$
\begin{aligned}
\left|M\left[\Psi_{j} e^{-r^{2}}\right](2 z+j+2)\right| & =\left|\int_{0}^{\infty} \Psi_{j}(x) e^{-x^{2}} x^{2 z+j+1} d x\right| \\
& \leqslant c \int_{0}^{1} e^{-x^{2}} x^{2 \sigma-c+j+1} d x+c \int_{1}^{\infty} e^{-x^{2}} x^{2 \sigma+c+j+1} d x \\
& \leqslant 2 c \Gamma\left(\sigma+\frac{j}{2}+\frac{c}{2}+1\right),
\end{aligned}
$$

where the above inequality holds for $\sigma>0$ sufficiently large. From this we obtain

$$
\left|\mathcal{F}_{j}(z)\right| \leqslant \frac{2 c \Gamma\left(\sigma+\frac{j}{2}+\frac{c}{2}+1\right)}{\exp \{(\sigma+s) \log (|z|)-\sigma-t \cdot \arg (z)+O(1)\} \times\left\{1+o\left(\frac{1}{|z|}\right)\right\}} .
$$

By Proposition 2.4.2, we know that $\mathcal{F}_{j}(z)$ is a $\delta$-periodic entire function. In order to estimate the growth of $\left|\mathcal{F}_{j}(z)\right|$ as $|z| \longrightarrow \infty$, we therefore can assume that in the above estimate $\sigma$ is large and varying in an interval of length $\delta$. According to (2.4.9), there is a constant $C>0$ such that

$$
\left|\mathcal{F}_{j}(z)\right| \leqslant \frac{C}{1+t^{2}} e^{t \arg (z)}<\frac{C}{1+t^{2}} e^{\frac{\pi}{2}|z|}
$$

Now, by applying Lemma 2.4.1, it follows that there are $a_{l} \in \mathbb{C}$ such that

$$
M\left[\Psi_{j} e^{-r^{2}}\right](2 z+j+2)=G_{j}(z) \sum_{|l|<\frac{\delta}{4}} a_{l} e^{\frac{2 \pi i l z}{\delta}} .
$$

Theorem 2.4.1. Let $\Psi \in S$ and suppose that $T_{f_{m, \delta}} T_{\Psi}=T_{\Psi} T_{f_{m, \delta}}$. Then for each $j>\delta$ there exists a trigonometric polynomial $\sum_{|l|<\frac{\delta}{4}} a_{l} e^{\frac{2 \pi i l z}{\delta}}$ such that:

$$
\Psi_{j}(r)=2 M^{-1}\left[G_{j}(z) \sum_{|l|<\frac{\delta}{4}} a_{l} e^{\frac{2 \pi i l z}{\delta}}\right]\left(r^{2}\right) r^{-j-2} e^{r^{2}} .
$$


Proof. According to (2.4.5) the transformation rule for the Mellin transform gives:

$$
M\left[r^{j+2} \Psi_{j} e^{-r^{2}}\right](2 z)=\frac{1}{2} M\left[r^{\frac{j}{2}+1} \Psi_{j}(\sqrt{r}) e^{-r}\right](z)=G_{j}(z) \sum_{|l|<\frac{\delta}{4}} a_{l} e^{\frac{2 \pi i l z}{\delta}} .
$$

Applying the inverse Mellin transform to both sides of the equation shows:

$$
\Psi_{j}(\sqrt{r})=2 M^{-1}\left[G_{j}(z) \sum_{|l|<\frac{\delta}{4}} e^{\frac{2 \pi i l z}{\delta}}\right](r) r^{-\frac{j}{2}-1} e^{r}
$$

and replacing $\sqrt{r}$ by $r$ proves the assertion.

Remark 2.4.1. Arguments analogous to Propositions 2.4.1, 2.4.2, 2.4.3, 2.4.5 and Theorem 2.4.1 were used in [66] to prove Theorem A0. Roughly speaking, in [66] and under the assumption that $T_{\varphi}$ and $T_{\psi}$ commute on $A(\mathbb{D})$, for each fixed $j \in \mathbb{Z}$ the author introduced the function

$$
F_{j}(z):=\frac{\Gamma\left(\frac{z+j}{2 \delta}\right) \Gamma\left(\frac{z+m+\delta-j}{2 \delta}\right)}{\Gamma\left(\frac{z+2 \delta-j}{2 \delta}\right) \Gamma\left(\frac{z+m+\delta+j}{2 \delta}\right)} .
$$

Using Equation (2.3.4) it follows that the function $\frac{M\left[\psi_{j}\right](z)}{F_{j}(z)}$ is $2 \delta$-periodic on some some right half-plane and hence can be extended to the whole complex plane forming an entire $2 \delta$-periodic function. Moreover, by a similar argument to that in Proposition 2.4.3 it was shown that $\frac{M\left[\psi_{j}\right](z)}{F_{j}(z)}=o(|z|)$ which means there is a constant $a_{0}$ such that equation $M\left[\psi_{j}\right]=a_{0} F_{j}(z)$ and Equation 2.1.1 follows. Furthermore, by studying estimating the growth of the inverse Mellin transform $M^{-1}\left[F_{j}\right]$ at infinity it follows that the function $\psi_{j}$ given by (2.1.1) is bounded and Theorem $A 0$ then follows.

Compared to the problem considered in this chapter, we are not able to prove in general that the functions given by (2.4.10) are of polynomial growth. However, the function $\phi_{j}(r):=$ $M^{-1}\left[G_{j}\right]\left(r^{2}\right) r^{-j-2} e^{r^{2}} \in S$ and satisfies $\left[T_{\phi_{j} e^{i j \theta}}, T_{f_{m, \delta}}\right]=0$. In some cases we are able to show that there is one and only one function namely $\varphi_{j} \in S$ such that $\left[T_{\phi_{j} e^{i j \theta}}, T_{f_{m, \delta}}\right]=0$. If we allow operator symbols of higher growth at infinity it turns out that there is more than one function $\psi_{j}$ with $\left[T_{\psi_{j} e^{i j \theta}}, T_{f_{m, \delta}}\right]=0$.

Fix $f_{m, \delta}, m \in \mathbb{R}_{+}, \delta \geqslant 0$, and for $j>\delta$, consider $G_{j}(z)$ which clearly defines a holomorphic function on $\operatorname{Re}(z)>0$. Suppose for a moment we found some trigonometric polynomials $\sum_{|l|<\frac{\delta}{4}} a_{l} e^{\frac{2 \pi i l z}{\delta}}$ such that

$$
\varphi_{j}(r) e^{i j \theta}:=M^{-1}\left[G_{j}(z) \sum_{|l|<\frac{\delta}{4}} a_{l} e^{\frac{2 \pi i l z}{\delta}}\right]\left(r^{2}\right) r^{-j-2} e^{r^{2}} e^{i j \theta},
$$

defines an element in $\mathcal{E}$. Then the equality (2.3.1) is satisfied if we replace $\Psi_{j}$ by $\varphi_{j}$, and so we find a collection of symbols $\left\{\varphi_{j}(r) e^{i j \theta}\right\}_{j>\delta} \subset \mathcal{E}$ such that $\left[T_{f_{m, \delta}}, T_{\varphi_{j} e^{i j \theta}}\right]=0$.

In this part of the chapter, we determine such a collection whenever $f_{m, \delta}$ is a monomial i.e. $p:=\frac{m+\delta}{2}, \frac{m-\delta}{2} \in \mathbb{N}_{0}$. Moreover, we prove that there is an infinite number of $j \in \mathbb{Z}$ such that 
$\left\{\varphi_{j}(r) e^{i j \theta}\right\} \subset S$ if and only if the trigonometricpolynomial in (2.4.10) is a constant. More precisely, for infinitely many $j \in \mathbb{Z}$ and up to a constant factor there is one function $\Psi_{j}(r) e^{i j \theta} \in$ $S$ such that $\left[T_{f_{m, \delta}}, T_{\Psi_{j} e^{i j \theta}}\right]=0$.

First we need to simplify the expression

$$
R_{j}(z):=\prod_{l=1}^{j} \Gamma\left(\frac{z+p+l}{\delta}\right) \prod_{\ell=1}^{j-\delta}\left[\Gamma\left(\frac{z+\delta+\ell}{\delta}\right)\right]^{-1}=G_{j}(z) \delta^{-z} .
$$

to a product of holomorphic polynomials and Gamma functions.

The case $p=\delta$ means that $f_{\delta, \delta}=z^{p}$ is holomorphic and $R_{j}(z)$ takes the form:

$$
R_{j}(z)=\prod_{l=j-\delta+1}^{j} \Gamma\left(\frac{z+p+l}{\delta}\right)=\prod_{l=0}^{\delta-1} \Gamma\left(\frac{z+j+1}{\delta}+\frac{l}{\delta}\right) .
$$

Now, assume that $p \in \mathbb{N}_{0}$ and consider the cases:

1. Case $j>p$ : Choose $q, n \in \mathbb{N}$ with $1 \leqslant q \leqslant \delta$ such that $j=n \delta+q$. Therefore:

(a) For each $\ell$ such that $1 \leqslant \ell \leqslant p-\delta$, choose $l=\ell+j-p+\delta-q$. Then $1+j-p+\delta-q \leqslant$ $l \leqslant j-q$, and

$$
\frac{\Gamma\left(\frac{z+p+l}{\delta}\right)}{\Gamma\left(\frac{z+\delta+\ell}{\delta}\right)}=\frac{\Gamma\left(\frac{z+\ell}{\delta}+1+n\right)}{\Gamma\left(\frac{z+\ell}{\delta}+1\right)}=\prod_{r=1}^{n}\left(\frac{z+\ell}{\delta}+r\right) .
$$

(b) In the case $p-\delta<\ell \leqslant j-\delta$ put $l=\ell-p+\delta$. Then $1 \leqslant l \leqslant j-p$ and

$$
\frac{\Gamma\left(\frac{z+p+l}{\delta}\right)}{\Gamma\left(\frac{z+\delta+\ell}{\delta}\right)}=1
$$

Therefore:

$$
R_{j}(z)=\prod_{\ell=1}^{p-\delta} \prod_{r=1}^{n}\left(\frac{z+\ell}{\delta}+r\right) \prod_{l=j-p+1}^{j-p+\delta-q} \Gamma\left(\frac{z+p+l}{\delta}\right) \prod_{l=j-q+1}^{j} \Gamma\left(\frac{z+p+l}{\delta}\right) .
$$

2. Case $j \leqslant p$ : Let $p=n \delta+q$ with $q, n \in \mathbb{N}$ and $1 \leqslant q \leqslant \delta$. Then for each $1 \leqslant \ell \leqslant j-\delta$, let $l=\ell+\delta-q$. Hence $1+\delta-q \leqslant l \leqslant j-q$ and

$$
\frac{\Gamma\left(\frac{z+p+l}{\delta}\right)}{\Gamma\left(\frac{z+\delta+\ell}{\delta}\right)}=\frac{\Gamma\left(\frac{z+\ell}{\delta}+n+1\right)}{\Gamma\left(\frac{z+\ell}{\delta}+1\right)}=\prod_{r=1}^{n}\left(\frac{z+\ell}{\delta}+r\right),
$$

which implies that

$$
R_{j}(z)=\prod_{\ell=1}^{j-\delta} \prod_{r=1}^{n}\left(\frac{z+\ell}{\delta}+r\right) \prod_{l=1}^{\delta-q} \Gamma\left(\frac{z+p+l}{\delta}\right) \prod_{l=j-q+1}^{j} \Gamma\left(\frac{z+p+l}{\delta}\right) .
$$


Proposition 2.4.4. Let $f_{m, \delta}$ be a monomial with $\delta>0$, and let $l \in \mathbb{Z}$ be fixed such that $|l|<\frac{\delta}{4}$. For each $j \in \mathbb{N}$ such that $j>\delta$ consider $G_{j}(z)=R_{j}(z) \delta^{z}$. Then:

(1) $G_{j}(z)$ is holomorphic on $\operatorname{Re}(z)>-j-1$.

(2) The inverse Mellin transform $M^{-1}\left[G_{j}(z) e^{\frac{2 \pi i l z}{\delta}}\right](x)$ exists for all $x>0$.

(3) $M^{-1}\left[G_{j}(z) e^{\frac{2 \pi i l z}{\delta}}\right]\left(x^{2}\right) x^{-j-2}=o\left(x^{j-\epsilon}\right)$ as $x \longrightarrow 0$.

Proof. (1) It follows directly by calculating the largest pole of $R_{j}(z)$ from (2.4.11), (2.4.12) and (2.4.13). In particular, if $p=\delta$ or $j>p$ then the largest pole is $-j-1$. If $j<p$ then it is located at $-p-1<-j-1$.

(2) For $z=\sigma+i t$ with $\sigma$ fixed and using (2.4.7) we see that there are constants $B$ and $s$ independent of $t$ such that

$$
\left|G_{j}(z)\right| \leq B \exp \left\{(\sigma+s) \log (|z|)-|t| \cdot \frac{\pi}{2}\right\} .
$$

Now, let $\sigma>-j-1$ be fixed, then it follows for $|l|<\frac{\delta}{4}$ :

$$
\begin{gathered}
\left|M^{-1}\left[G_{j}(z) e^{\frac{2 \pi i l z}{\delta}}\right](x)\right|=\left|\frac{1}{2 \pi i} \int_{\sigma-i \infty}^{\sigma+i \infty} x^{-z} G_{j}(z) e^{\frac{2 \pi i l z}{\delta}} d z\right| \\
\leqslant \frac{B}{2 \pi} x^{-\sigma} e^{-\sigma} \int_{-\infty}^{\infty}\left(\sigma^{2}+t^{2}\right)^{\frac{\sigma+s}{2}} e^{-|t| \cdot \frac{\pi}{2}-\frac{2 \pi l t}{\delta}} d t<\infty .
\end{gathered}
$$

(3) For a positive number $\epsilon$ we put $\sigma=-j-1+\frac{\epsilon}{2}$. Then:

$$
\left|M^{-1}\left[G_{j}(z) e^{\frac{2 \pi i l z}{\delta}}\right]\left(x^{2}\right)\right| x^{-j-2} \leq \frac{1}{2 \pi} x^{-2 \sigma-j-2} \int_{\mathbb{R}}\left|G_{j}(\sigma+i t)\right| e^{\frac{-2 \pi l t}{\delta}} d t .
$$

Now, we give a global estimate for the inverse of the Mellin transform:

Proposition 2.4.5. Let $l \in \mathbb{Z}$ be fixed with $|l|<\frac{\delta}{4}$. Then for each $j>\delta$ there exists a constant c such that:

$$
\left|M^{-1}\left[G_{j}(z) e^{\frac{2 \pi i l z}{\delta}}\right]\left(r^{2}\right) r^{-j-2} e^{r^{2}}\right| \leqslant c r^{2 s-j+3} e^{r^{2}\left(1-\cos \left(\frac{2 \pi l}{\delta}\right)\right)} .
$$

Proof. Throughout the proof $c$ denotes some constant which may change from line to line. For each $n \in \mathbb{N}$ we have

$$
\left|M^{-1}\left[G_{j}(z) e^{\frac{2 \pi i l z}{\delta}}\right](x)\right|=\left|\frac{1}{2 \pi i} \int_{n-i \infty}^{n+i \infty} x^{-z} G_{j}(z) e^{\frac{2 \pi i l z}{\delta}} d z\right| .
$$

Together with (2.4.7) it follows that

$$
\begin{aligned}
\mid x^{n} M^{-1}\left[G_{j}(z) e^{\left.\frac{2 \pi i l z}{\delta}\right]}(x) \mid\right. & \leqslant \frac{1}{2 \pi} \int_{\mathbb{R}}\left|G_{j}(n+i t)\right| e^{-\frac{2 \pi l t}{\delta}} d t \\
& \leqslant c \int_{\mathbb{R}} e^{n \log \sqrt{n^{2}+t^{2}}-t \cdot \arctan \frac{t}{n}+s \log \sqrt{n^{2}+t^{2}}-n-\frac{2 \pi l t}{\delta}} d t \\
& =c e^{n \log n-n} n^{s} \int_{\mathbb{R}} e^{n \log \sqrt{1+\frac{t^{2}}{n^{2}}}-t \cdot \arctan \frac{t}{n}+s \log \sqrt{1+\frac{t^{2}}{n^{2}}-\frac{2 \pi l t}{\delta}} d t} \\
& =c e^{n \log n-n} n^{s+1} \int_{\mathbb{R}} e^{n\left[\log \sqrt{1+u^{2}}-u \arctan u-\frac{2 \pi l u}{\delta}\right]+s \log \sqrt{1+u^{2}}} d u
\end{aligned}
$$


where we have used the change of variable $u=\frac{t}{n}$. Let $H_{l}(u)$ be the function on $\mathbb{R}$ defined by

$$
H_{l}(u):=\log \sqrt{1+u^{2}}-u \arctan u-\frac{2 \pi l u}{\delta} .
$$

Then $H_{l}(u)$ attains its maximum at $u_{0}=-\tan \frac{2 \pi l}{\delta}$ with $H_{l}\left(u_{0}\right)=-\log \cos \frac{2 \pi l}{\delta}$. Moreover, it is easy to check that $\lim _{u \rightarrow+\infty} H_{l}(u)=\lim _{u \rightarrow-\infty} H_{l}(u)=-\infty$.

Now, let $r>0$ be such that $H_{l}(u) \leqslant-1$ for $|u|>r$. Then we have for all $n \in \mathbb{N}$ :

(1) $\int_{-\infty}^{-r} e^{n H_{l}(u)+s \log \sqrt{1+u^{2}}} d u \leqslant \int_{-\infty}^{-r} \exp \left\{H_{l}(u)+s \log \sqrt{1+u^{2}}\right\} d u \leqslant c$,

(2) $\int_{r}^{\infty} e^{n H_{l}(u)+s \log \sqrt{1+u^{2}}} d u \leqslant \int_{r}^{\infty} \exp \left\{H_{l}(u)+s \log \sqrt{1+u^{2}}\right\} d u \leqslant c$,

(3) $\int_{-r}^{r} e^{n H_{l}(u)+s \log \sqrt{1+u^{2}}} d u \leqslant c e^{-n \log \cos \frac{2 \pi l}{\delta}}$.

By substituting (1)-(3) into the above inequality, we obtain

$$
\left|x^{n} M^{-1}\left[G_{j}(z) e^{\frac{2 \pi i l z}{\delta}}\right](x)\right| \leqslant c n^{s+1} \frac{e^{n \log n-n}}{\left[\cos \left(\frac{2 \pi l}{\delta}\right)\right]^{n}} .
$$

According to Stirling's formula we know that

$$
e^{n \log n-n} \sim \frac{n !}{\sqrt{2 \pi n}}, \quad \text { as } n \longrightarrow \infty
$$

and it follows that

$$
n^{-s-\frac{5}{2}} \frac{x^{n} \cos ^{n}\left(\frac{2 \pi l}{\delta}\right)}{n !}\left|M^{-1}\left[G_{j}(z) e^{\frac{2 \pi i l z}{\delta}}\right](x)\right| \leqslant \frac{c}{n^{2}} .
$$

Note that for each $q>0$ there exists $d>0$ such that for all $x>d$ we have

$$
x^{-q} e^{x} \leqslant \sum_{n \in \mathbb{N}} \frac{1}{n^{q}} \frac{x^{n}}{n !} .
$$

Using this estimate and summing up the inequalities (2.4.14) over $n \in \mathbb{N}$ we get

$$
\begin{aligned}
x^{-s-\frac{5}{2}} e^{x \cos \frac{2 \pi l}{\delta}}\left|M^{-1}\left[G_{j}(z) e^{\frac{2 \pi i l z}{\delta}}\right](x)\right| & \leqslant \sum_{n \in \mathbb{N}} n^{-s-\frac{5}{2}} x^{n} \cos ^{n}\left(\frac{2 \pi l}{\delta}\right)\left|M^{-1}\left[G_{j}(z) e^{\frac{2 \pi i l z}{\delta}}\right](x)\right| \\
& \leqslant \sum_{n \in \mathbb{N}} \frac{c}{n^{2}}<\infty
\end{aligned}
$$

Finally, the assertion follows by replacing $x$ with $r^{2}$.

As a consequence of the above proposition, we obtain:

Corollary 2.4.1. Let $f_{m, \delta}\left(r e^{i \theta}\right)=r^{m} e^{i \delta \theta}$ be a monomial, and let $l \in \mathbb{Z}$ such that $|l|<$ $\frac{\delta}{2 \pi} \arccos \frac{3}{4}$. Then for each $j>\delta$ the function

$$
\varphi_{j}(r) e^{i j \theta}:=M^{-1}\left[G_{j}(z) e^{\frac{2 \pi i l z}{\delta}}\right]\left(r^{2}\right) r^{-j-2} e^{r^{2}} e^{i j \theta},
$$

defines an element in $\mathcal{E}$ and satisfies $\left[T_{f_{m, \delta}}, T_{\varphi_{j} e^{i j \theta}}\right]=0$. Moreover, $\varphi_{j}(r) e^{i j \theta} \in S$ in case $l=0$. 
By applying the duplication formula for the Gamma function for $k \in \mathbb{N}$ :

$$
\prod_{l=0}^{k-1} \Gamma\left(z+\frac{l}{k}\right)=(2 \pi)^{\frac{k-1}{2}} k^{\frac{1}{2}-k z} \Gamma(k z)
$$

we give several examples where $G_{j}(z)$ takes the form $G_{j}(z)=\wp(z) \Gamma(z)$ with $\wp(z) \in \mathbb{P}[z]$.

Example 2.4.2. (1): Suppose that $f_{m, \delta}$ is holomorphic i.e. $m=\delta$. Then applying the above duplication formula to Equation (2.4.11) we obtain

$$
\begin{aligned}
R_{j}(z) & =\prod_{l=0}^{\delta-1} \Gamma\left(\frac{z+j+1}{\delta}+\frac{l}{\delta}\right) \\
& =(2 \pi)^{\frac{\delta-1}{2}} \delta^{-z-j-\frac{1}{2}} \Gamma(z+j+1) \\
& =(2 \pi)^{\frac{\delta-1}{2}} \delta^{-z-j-\frac{1}{2}} \prod_{l=0}^{j}(z+l) \Gamma(z) .
\end{aligned}
$$

(2): As before we write $p:=\frac{m+\delta}{2}$. Now, let $j>p \geqslant \delta$ with $j=n \delta$ for some $n \in \mathbb{N}$. Then using (2.4.12) we get

$$
\begin{aligned}
R_{j}(z) & =\prod_{\ell=1}^{p-\delta} \prod_{r=1}^{n-1}\left(\frac{z+\ell}{\delta}+r\right) \prod_{l=j-\delta+1}^{j} \Gamma\left(\frac{z+p+l}{\delta}\right) \\
& =\prod_{\ell=1}^{p-\delta} \prod_{r=1}^{n-1}\left(\frac{z+\ell}{\delta}+r\right) \prod_{l=0}^{\delta-1} \Gamma\left(\frac{z+p+j-\delta+1}{\delta}+\frac{l}{\delta}\right) \\
& =(2 \pi)^{\frac{\delta-1}{2}} \delta^{-z-p-j+\delta-\frac{1}{2}} \Gamma(z+p+j-\delta+1) \prod_{\ell=1}^{p-\delta} \prod_{r=1}^{n-1}\left(\frac{z+\ell}{\delta}+r\right) \\
& =(2 \pi)^{\frac{\delta-1}{2}} \delta^{-z-p-j+\delta-\frac{1}{2}}\left[\prod_{\ell=1}^{p-\delta} \prod_{r=1}^{n-1}\left(\frac{z+\ell}{\delta}+r\right)\right]\left[\prod_{l=0}^{p+j-\delta}(z+l)\right] \Gamma(z) .
\end{aligned}
$$

(3): Suppose that $p=n \delta$ for some $n \in \mathbb{N}$ with $n>1$, which is equivalent to $m=(2 n-1) \delta$.

Then for each $j$ such that $\delta<j \leq p$ and using (2.4.13) we have

$$
\begin{aligned}
R_{j}(z) & =\prod_{\ell=1}^{j-\delta} \prod_{r=1}^{n-1}\left(\frac{z+\ell}{\delta}+r\right) \prod_{l=j-\delta+1}^{j} \Gamma\left(\frac{z+p+l}{\delta}\right) \\
& =\prod_{\ell=1}^{j-\delta} \prod_{r=1}^{n-1}\left(\frac{z+\ell}{\delta}+r\right) \prod_{l=0}^{\delta-1} \Gamma\left(\frac{z+p+j-\delta+1}{\delta}+\frac{l}{\delta}\right) \\
& =(2 \pi)^{\frac{\delta-1}{2}} \delta^{-z-p-j+\delta-\frac{1}{2}}\left[\prod_{\ell=1}^{j-\delta} \prod_{r=1}^{n-1}\left(\frac{z+\ell}{\delta}+r\right)\right]\left[\prod_{l=0}^{p+j-\delta}(z+l)\right] \Gamma(z) .
\end{aligned}
$$

Corollary 2.4.2. Let $\Psi \in S$ and let $f_{m, \delta}=r^{m} e^{i \delta \theta}$ be a monomial. Suppose that $T_{f_{m, \delta}} T_{\Psi}=$ $T_{\Psi} T_{f_{m, \delta}}$ and that one of the following cases holds: 
(1) $f_{m, \delta}$ is holomorphic,

(2) $j>p$, and $j=n \delta$ for some $n \in \mathbb{N}$,

(3) $\delta<j<p$, and $p=n \delta$.

Then there is a constant $a_{0} \in \mathbb{C}$ such that

$$
\Psi_{j}(r)=a_{0} M^{-1}\left[G_{j}(z)\right]\left(r^{2}\right) r^{-j-2} e^{r^{2}} \in S .
$$

Proof. The above cases occur in the equations (2.4.15), (2.4.16) and (2.4.17). Therefore, we have $G_{j}(z)=\wp(z) \Gamma(z)$ where $\wp(z)$ is a polynomial in $z$. According to Theorem 2.4.1 we know that

$$
\Psi_{j}(r)=2 M^{-1}\left[G_{j}(z) \sum_{|l|<\frac{\delta}{4}} a_{l} e^{\frac{2 \pi i l z}{\delta}}\right]\left(r^{2}\right) r^{-j-2} e^{r^{2}}
$$

For each $l$ such that $|l|<\frac{\delta}{4}$ we have (c.f. [154], and (2.7.2) below):

$$
M^{-1}\left[\Gamma(z) e^{\frac{2 \pi i l z}{\delta}}\right](r)=e^{-r e^{-\frac{2 \pi i l}{\delta}}}
$$

and therefore

$$
M^{-1}\left[G_{j}(z) e^{\frac{2 \pi i l z}{\delta}}\right](r)=\wp_{1}^{l}(r) e^{-r e^{-\frac{2 \pi i l}{\delta}}}
$$

where $\wp_{1}^{l}(r)$ is a non-zero polynomial in $r$ and having values in $\mathbb{C}$. This means that

$$
\frac{1}{2} \Psi_{j}(r)=r^{-j-2} a_{0} \wp_{0}^{l}\left(r^{2}\right)+r^{-j-2} e^{r^{2}} \sum_{|l| \neq 0} a_{l} \wp_{1}^{l}\left(r^{2}\right) e^{-r^{2} e^{-\frac{2 \pi i l}{\delta}}} .
$$

Now we want to prove that

$$
\Psi_{j}(r) \in S \Longleftrightarrow a_{l}=0 \text { for all } l \neq 0
$$

Let $0<k<\frac{\delta}{4}$ be the greatest positive integer such that $a_{k} \neq 0$ or $a_{-k} \neq 0$. Then (2.4.18) can be written in the form

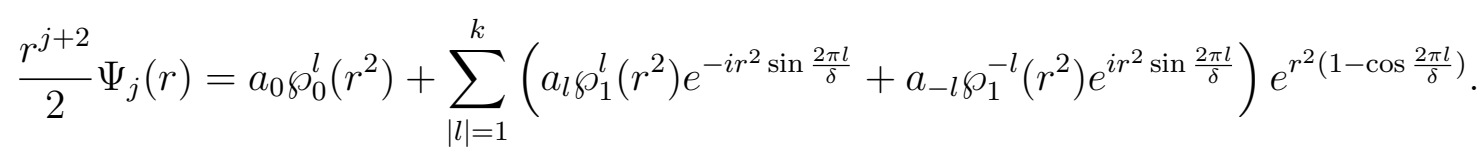

Note that given positive numbers $\alpha_{1}<\alpha_{2}<\cdots<\alpha_{m}$ and radial functions $\varphi_{1}, \cdots, \varphi_{m}$ it is easy to see that if $\sum_{\ell=1}^{m} \varphi_{\ell}(r) e^{\alpha_{\ell} r^{2}} \in S$ then $\varphi_{m}(r) \longrightarrow 0$ as $r \longrightarrow \infty$. Since $\Psi_{j} \in S$ by assumption this shows that

$$
\lim _{r \longrightarrow \infty}\left(a_{k} \wp_{1}^{k}\left(r^{2}\right) e^{-i r^{2} \sin \frac{2 \pi k}{\delta}}+a_{-k} \wp_{1}^{-k}\left(r^{2}\right) e^{i r^{2} \sin \frac{2 \pi k}{\delta}}\right)=0 .
$$

In particular, this holds true for the sequence $r_{n}:=\sqrt{\frac{2 n \pi}{\sin \frac{2 \pi k}{\delta}}}$ so that

$$
\lim _{n \longrightarrow \infty}\left(a_{k} \wp_{1}^{k}\left(r_{n}^{2}\right)+a_{-k} \wp_{1}^{-k}\left(r_{n}^{2}\right)\right)=0 .
$$


This shows that $a_{k} \wp_{1}^{k}\left(r^{2}\right)+a_{-k} \wp_{1}^{-k}\left(r^{2}\right) \equiv 0$. By substituting this relation into (2.4.19) we obtain as $r \longrightarrow \infty$

$$
a_{k} \wp_{1}^{k}\left(r^{2}\right)\left[e^{-i r^{2} \sin \frac{2 \pi k}{\delta}}-e^{i r^{2} \sin \frac{2 \pi k}{\delta}}\right]=-2 i a_{k} \wp_{1}^{k}\left(r^{2}\right) \sin \left(r^{2} \sin \frac{2 \pi k}{\delta}\right) \longrightarrow 0 .
$$

In particular, if we choose the sequence $r_{q}=\sqrt{\frac{(4 q+1) \pi}{2 \sin \frac{2 \pi k}{\delta}}}$ where $q$ is any positive integer, then

$$
\lim _{q \longrightarrow \infty} a_{k} \wp_{1}^{k}\left(r_{q}^{2}\right)=0 \text {. }
$$

Therefore, $a_{k} \wp_{1}^{k}\left(r^{2}\right) \equiv 0$ i.e. $a_{k}=a_{-k}=0$ which is a contradiction.

Remark 2.4.2. For $s>0$ consider the Segal-Bargmann space $\mathcal{H}_{s}^{2}$ over $\mathbb{C}$ with respect to the Gaussian measure $d \mu_{(s)}(z):=\frac{s}{\pi} e^{-s|z|^{2}} d v(z)$. The Toeplitz operator on $\mathcal{H}_{s}^{2}$ with symbol $h$ is denoted by $\mathcal{T}_{h}^{s}$. Note that for every $g \in \mathcal{H}_{s}^{2}$ s.t. $h g \in L^{2}\left(\mathbb{C}, d \mu_{(s)}\right)$ we have

$$
\mathcal{T}_{h}^{s} g(z)=\left[T_{h\left(\frac{\dot{\sqrt{s}}}{s}\right.} g\left(\frac{\cdot}{\sqrt{s}}\right)\right](\sqrt{s} z),
$$

where $T_{h\left(\frac{\dot{\sqrt{s}}}{}\right)}$ corresponds to the Toeplitz operator on $H^{2}$ with symbol $h\left(\frac{\dot{\sqrt{s}}}{)}\right.$. Now let us consider two monomials $f$ and $g$. It is well known (c.f. [14, 63]) that the product operator

$$
\mathcal{T}_{f}^{s} \mathcal{T}_{g}^{s}=\mathcal{T}_{f \sharp_{s} g}^{s}, \quad \text { for any two monomials } f \text { and } g,
$$

where $f \sharp_{s} g$ is the polynomial given by

$$
f \sharp_{s} g(z)=\sum_{k \in \mathbb{N}} \frac{1}{(-s)^{k} k !}\left(d_{z}^{k} f\right)(z)\left(d_{\bar{z}}^{k} g\right)(z) .
$$

Note that Equation (2.4.21) holds true for more general symbols (c.f. Lemma 16 in [14]).

Suppose that the Toeplitz operators $T_{f}$ and $T_{g}$ commute on the space of holomorphic polynomials in $H^{2}$. Then by (2.4.20) and (2.4.21) one can easily verify that the operators $T_{f}^{s}$ and $T_{g}^{s}$ commute on the holomorphic polynomials in $\mathcal{H}_{s}^{2}$ for all $s>0$.

If we fix $f=f_{m, \delta}$ as a monomial and $g=\psi_{j}(r) e^{i j \theta}$ of polynomial growth at infinity (not necessarily monomial) for some $j>\delta$, one may ask wether the condition $\left[T_{f_{m, \delta}}, T_{\psi_{j}(r) e^{i j \theta}}\right]=0$ on $\mathbb{P}[z]$ still implies that $\left[\mathcal{T}_{f_{m, \delta}^{s}}^{s}, \mathcal{T}_{\psi_{j}(r) e^{i j \theta}}^{s}\right]=0$ on $\mathbb{P}[z]$ for all $s>0$. This is in general not true. In particular, the Toeplitz operators $\mathcal{T}_{f}^{s}$ and $\mathcal{T}_{g}^{s}$ with symbols

$$
f(z)=f_{m, \delta}(z)=z^{2} \bar{z}=r^{3} e^{i \theta}, \text { and } g(z)=\psi_{2}(r) e^{i \theta}=\left(r^{6}-2 r^{4}\right) e^{2 i \theta}
$$

commute on $\mathbb{P}[z] \subset \mathcal{H}_{s}^{2}$ if and only if $s=1$ (c.f. Appendix A.2.1 for the proof).

\subsection{Case $j<0$}

In this section we consider operator symbols $\Psi \in S$ and $f_{m, \delta}=r^{m} e^{i \delta \theta}$ with $m \in \mathbb{R}_{+}, \delta \in \mathbb{N}$. Under the assumption $T_{f_{m, \delta}} T_{\Psi}=T_{\Psi} T_{f_{m, \delta}}$, we aim to determine the functions $\Psi_{j}(r)$ of the expansion in Lemma 2.2.1 whenever $j<0$. An important result here is that $\Psi_{j} \equiv 0$ in the case $j<-2\left[\frac{\delta}{4}\right]$ where [.] is the greatest integer function. Similar to Proposition 4 (c.f. Appendix A.2.2 for a proof), we have: 
Proposition 2.5.1. Let $\Psi \in S$ and suppose that $T_{f_{m, \delta}} T_{\Psi}=T_{\Psi} T_{f_{m, \delta}}$. Then for each $j<0$ the following functional equations:

$$
M\left[\Psi_{j} e^{-r^{2}}\right](2 z+2 \delta+j+2)=M\left[\Psi_{j} e^{-r^{2}}\right](2 z+j+2) \prod_{l=1}^{\delta-j}(z+j+l) \prod_{l=1}^{-j}\left(z+j+\frac{\delta+m}{2}+l\right)^{-1}
$$

hold in the half plane $\operatorname{Re}(z)>-j-1$.

Fix $j<0$ and consider the meromorphic function:

$$
H_{j}(z)=\prod_{l=1}^{\delta-j} \Gamma\left(\frac{z+j+l}{\delta}\right) \prod_{l=1}^{-j}\left[\Gamma\left(\frac{z+j+\frac{\delta+m}{2}+l}{\delta}\right)\right]^{-1} \delta^{z}
$$

Note that the quotient

$$
\tau_{j}(z):=\frac{M\left[\Psi_{j} e^{-r^{2}}\right](2 z+j+2)}{H_{j}(z)}
$$

is holomorphic and well defined on the half plane $\operatorname{Re}(z)>-j-1$. By (2.5.1) it is easy to check that $\tau_{j}(z)$ is $\delta$-periodic (c.f. the proof of Proposition 2.4.2). Therefore, it can be extended to an entire function on the whole complex plane. As an analog to Proposition 2.4.3 (c.f. Appendix A.2.3for a proof), we conclude

Proposition 2.5.2. Let $\Psi \in S$ and suppose that $T_{f_{m, \delta}} T_{\Psi}=T_{\Psi} T_{f_{m, \delta}}$. Then for each $j<0$ there exists a trigonometric polynomial $\sum_{|l|<\frac{\delta}{4}} a_{l} e^{\frac{2 \pi i l z}{\delta}}$ such that for $\operatorname{Re}(z)>-j-1$ we have

$$
M\left[\Psi_{j} e^{-r^{2}}\right](2 z+j+2)=H_{j}(z) \sum_{|l|<\frac{\delta}{4}} a_{l} e^{\frac{2 \pi i l z}{\delta}} .
$$

Assume that $\Psi \in S$ with (2.5.4). We know from Theorem 2.3.1 that for a fixed $j<0$ the assumption $M\left[\Psi_{j} e^{-r^{2}}\right](2 k+2 \delta+j+2)=0$ for all $k \in \mathbb{N}_{0}$ with $-\delta-j \leqslant k \leqslant-j-1$ is necessary and sufficient for the operators $T_{f_{m, \delta}}$ and $T_{\Psi_{j} e^{i j \theta}}$ to commute.

Theorem 2.5.1. Let $\Psi \in S$ and suppose that $T_{f_{m, \delta}} T_{\Psi}=T_{\Psi} T_{f_{m, \delta}}$, then for each $j \in \mathbb{Z}$ with $j<-2\left[\frac{\delta}{4}\right]$ we have $\Psi_{j} \equiv 0$.

Proof. Fix $j<-2\left[\frac{\delta}{4}\right]$ and for convenience write

$$
\tau_{j}(z):=\frac{M\left[\Psi_{j} e^{-r^{2}}\right](2 z+j+2)}{H_{j}(z)}=\sum_{|l| \leqslant\left[\frac{\delta}{4}\right]} a_{l} e^{\frac{2 \pi i l z}{\delta}}
$$

where $a_{\frac{\delta}{4}}=a_{-\frac{\delta}{4}}=0$ whenever $\delta \in 4 \mathbb{N}$. Taking condition (2.3.2) into account we have to consider two cases:

1. If $j \leqslant-\delta$, then according to (2.3.2) the Mellin transform $M\left[\Psi_{j} e^{-r^{2}}\right](2 z+j+2)$ vanishes at the points

$$
\{-j,-j+1, \cdots,-j+\delta-1\}
$$


Since $H_{j}(z)$ is well defined and nowhere zero on this set, condition (2.3.2) is equivalent to say that $\tau_{j}(z)$ vanishes exactly on

$$
\{-j,-j+1, \cdots,-j+\delta-1\} \text {. }
$$

This in turn, is equivalent to say that the trigonometric polynomial

$$
\tau_{j}\left(\frac{\delta}{2 \pi} z\right)=\sum_{|l| \leqslant\left[\frac{\delta}{4}\right]} a_{l} e^{i l z}
$$

vanishes at the $\delta$ points

$$
\left\{\frac{2 \pi}{\delta}(-j), \frac{2 \pi}{\delta}(-j+1), \cdots, \frac{2 \pi}{\delta}(-j+\delta-1)\right\} .
$$

From $\frac{2 \pi}{\delta}(\delta-1)<2 \pi$ and $2\left[\frac{\delta}{4}\right]<\delta$ we obtain $\tau_{j} \equiv 0$. (If $\tau_{j}\left(\frac{\delta}{2 \pi} z\right) \neq 0$, then it can have at most $2\left[\frac{\delta}{4}\right]$ roots in any interval of length less than $2 \pi$ ).

2. In the case of $-\delta<j<-2\left[\frac{\delta}{4}\right]$ condition (2.3.2) implies that $M\left[\Psi_{j} e^{-r^{2}}\right](2 k+2 \delta+j+2)$ vanishes for all $k \in\{0,1, \cdots,-j-1\}$. That is, $M\left[\Psi_{j} e^{-r^{2}}\right](2 z+j+2)$ vanishes at the $(-j)$-points

$$
\{\delta, \delta+1, \cdots, \delta-j-1\} .
$$

Again, note that $H_{j}(z)$ is well defined and nowhere zero on this set. We conclude that $\tau_{j}(z)$ vanishes at the points $\{\delta, \delta+1, \cdots, \delta-j-1\}$, which implies that

$$
\tau_{j}\left(\frac{\delta}{2 \pi} z\right)=0 \text { for all } z \in\left\{2 \pi, \frac{2 \pi}{\delta}(\delta+1), \cdots, \frac{2 \pi}{\delta}(\delta-j-1)\right\} .
$$

By assumption we have $\frac{2 \pi}{\delta}(-j-1)<2 \pi$ and $2\left[\frac{\delta}{4}\right]<-j$, which proves that $\tau_{j}(z) \equiv 0$.

Remark 2.5.1. In comparison to the case of the Bergman space over unit disc and with assumption considered in Theorem AO, I. Louhichi and L. Zakariasy proved that the condition $T_{\varphi} T_{\psi}=T_{\psi} T_{\varphi}$ on $A(\mathbb{D})$ implies that $\psi_{j}=0$ for all $j<0$ (c.f. Theorem 11 in [130]). Hence there is no non-zero quasi-homogeneous symbol $\psi_{j} e^{i j \theta}$ such that $\left[T_{\varphi}, T_{\psi_{j} e^{i j \theta}}\right]=0$ on $A(\mathbb{D})$. However, we are not able to prove this fact in our problem but we point out that if we allow operator symbol of higher growth then there are non-zero symbols in $\mathcal{E}$ whose corresponding Toeplitz operator commmute with $T_{f_{m, \delta}}$ whenever $\delta>\frac{2 \pi}{\arccos \frac{3}{4}}$ (c.f. Theorem 2.5.3 below).

Condition (2.3.2) and Proposition 2.5.2 lead to the following fact:

Theorem 2.5.2. Let $\Psi \in S$ and suppose that $T_{f_{m, \delta}} T_{\Psi}=T_{\Psi} T_{f_{m, \delta}}$. Then for each $j<0$ with $2\left[\frac{\delta}{4}\right]+j \geqslant 0$ there exists a trigonometric polynomial $\sum_{l=-\left[\frac{\delta}{4}\right]}^{\left[\frac{\delta}{4}\right]+j} \tilde{a}_{l} e^{\frac{2 \pi i l z}{\delta}}$ such that for $\operatorname{Re}(z)>$ $-j-1$ :

$$
M\left[\Psi_{j} e^{-r^{2}}\right](2 z+j+2)=H_{j}(z) \prod_{n=0}^{-j-1}\left(e^{\frac{2 \pi i z}{\delta}}-e^{\frac{2 \pi i n}{\delta}}\right) \sum_{l=-\left[\frac{\delta}{4}\right]}^{\left[\frac{\delta}{4}\right]+j} \tilde{a}_{l} e^{\frac{2 \pi i l z}{\delta}}
$$

where $\tilde{a}_{-\frac{\delta}{4}}=\tilde{a}_{\frac{\delta}{4}+j}=0$ whenever $\delta \in 4 \mathbb{N}$. 
Proof. By (2.3.2) we know that $M\left[\Psi_{j} e^{-r^{2}}\right](2 z+j+2)$ vanishes for

$$
z \in\{\delta, \delta+1, \cdots, \delta-j-1\}
$$

According to (2.5.4), and since $H_{j}(z)$ is well defined and nowhere zero on the above set, we conclude that $\{\delta, \delta+1, \cdots, \delta-j-1\}$ are zeros of the trigonometric polynomial $\tau_{j}(z)=$ $\sum_{|l| \leqslant\left[\frac{\delta}{4}\right]} a_{l} e^{\frac{2 \pi i l z}{\delta}}$ where $a_{\frac{\delta}{4}}=a_{-\frac{\delta}{4}}=0$ whenever $\delta \in 4 \mathbb{N}$. Note that

$$
\begin{aligned}
\tau_{j}(z)=\sum_{|l| \leqslant\left[\frac{\delta}{4}\right]} a_{l} e^{\frac{2 \pi i l z}{\delta}} & =\exp \left\{-\frac{2 \pi i z}{\delta}\left[\frac{\delta}{4}\right]\right\} \sum_{l=0}^{2\left[\frac{\delta}{4}\right]} a_{l-\left[\frac{\delta}{4}\right]} e^{\frac{2 \pi i l z}{\delta}} \\
& =\exp \left\{-\frac{2 \pi i z}{\delta}\left[\frac{\delta}{4}\right]\right\} P\left(e^{\frac{2 \pi i z}{\delta}}\right),
\end{aligned}
$$

where $P(w)$ is the following polynomial in the complex variable $w$ :

$$
P(w)=\sum_{l=0}^{2\left[\frac{\delta}{4}\right]} a_{l-\left[\frac{\delta}{4}\right]} w^{l} .
$$

Now, $\tau_{j}(z)$ vanishes at the $(-j)$-points $\{\delta, \delta+1, \cdots, \delta-j-1\}$ if and only if the polynomial $P(w)$ vanishes at $\left\{1, e^{\frac{2 \pi i}{\delta}}, \cdots, e^{\frac{2 \pi i}{\delta}(-j-1)}\right\} \subset \mathbb{S}^{1}$. This means $P(w)$ has the form

$$
P(w)=\prod_{n=0}^{-j-1}\left(w-e^{\frac{2 \pi i n}{\delta}}\right) \widetilde{P}(w)
$$

where $\widetilde{P}(w)$ is a polynomial of maximal degree $2\left[\frac{\delta}{4}\right]+j \in \mathbb{N}_{0}$. Therefore

$$
M\left[\Psi_{j} e^{-r^{2}}\right](2 z+j+2)=H_{j}(z) \widetilde{P}\left(e^{\frac{2 \pi i z}{\delta}}\right) e^{-\frac{2 \pi i z}{\delta}\left[\frac{\delta}{4}\right]} \prod_{n=0}^{-j-1}\left(e^{\frac{2 \pi i z}{\delta}}-e^{\frac{2 \pi i n}{\delta}}\right) .
$$

Proposition 2.5.3. Fix $\delta>0$, and let $j<0$ be such that $2\left[\frac{\delta}{4}\right]+j \geqslant 0$. Then for each $l \in \mathbb{Z}$ such that $-\frac{\delta}{4}<l<\frac{\delta}{4}+j$, we have:

(1) $H_{j}(z) \prod_{n=0}^{-j-1}\left(e^{\frac{2 \pi i z}{\delta}}-e^{\frac{2 \pi i n}{\delta}}\right)$ is holomorphic on $\operatorname{Re}(z)>-1$.

(2) The inverse Mellin transform $M^{-1}\left[H_{j}(z) e^{\frac{2 \pi i l z}{\delta}} \prod_{n=0}^{-j-1}\left(e^{\frac{2 \pi i z}{\delta}}-e^{\frac{2 \pi i n}{\delta}}\right)\right](x)$ exists for all $x>0$.

(3) $M^{-1}\left[H_{j}(z) e^{\frac{2 \pi i l z}{\delta}} \prod_{n=0}^{-j-1}\left(e^{\frac{2 \pi i z}{\delta}}-e^{\frac{2 \pi i n}{\delta}}\right)\right]\left(x^{2}\right) x^{-j-2}=o\left(x^{-j-\epsilon}\right)$ for all $\epsilon>0$ and as $x \rightarrow 0$.

Proof. (1): It is clear that $H_{j}(z)$ is holomorphic on $\operatorname{Re}(z)>-j-1$ and admits the simple poles $\{0,1, \cdots,-j-1\}$ in the region $\operatorname{Re}(z)>-1$. However, $\{0,1, \cdots,-j-1\}$ are roots of the trigonometric polynomial $\prod_{n=0}^{-j-1}\left(e^{\frac{2 \pi i z}{\delta}}-e^{\frac{2 \pi i n}{\delta}}\right)$. 
(2): The expansion of $H_{j}(z) e^{\frac{2 \pi i l z}{\delta}} \prod_{n=0}^{-j-1}\left(e^{\frac{2 \pi i z}{\delta}}-e^{\frac{2 \pi i n}{\delta}}\right)$ can be written as a linear combination of functions of the form $H_{j}(z) e^{\frac{2 \pi i \nu z}{\delta}}$ where $-\frac{\delta}{4}<\nu<\frac{\delta}{4}$. Now, the assertion follows in a way similar to the proof of Proposition 2.4.4, (2).

(3): This follows by an argument similar to the one in Proposition 2.4.4, (3) together with (1) and (2) above.

Theorem 2.5.3. Let $f_{m, \delta}\left(r e^{i \theta}\right)=r^{m} e^{i \delta \theta}$ with $\delta>\frac{2 \pi}{\arccos \frac{3}{4}}$. Then for each $j<0$ such that $j+\frac{\delta}{2 \pi} \arccos \frac{3}{4}>0$ and for each $l \in \mathbb{Z}$ such that $-\frac{\delta}{4}<l<\frac{\delta}{4}+j$ with $|l-j|<\frac{\delta}{2 \pi} \arccos \frac{3}{4}$ the function

$$
\varphi_{j}(r) e^{i j \theta}:=M^{-1}\left[H_{j}(z) \prod_{n=0}^{-j-1}\left(e^{\frac{2 \pi i z}{\delta}}-e^{\frac{2 \pi i n}{\delta}}\right) e^{\frac{2 \pi i l z}{\delta}}\right]\left(r^{2}\right) r^{-j-2} e^{r^{2}} e^{i j \theta}
$$

defines an element in $\mathcal{E}$ and satisfies $\left[T_{f_{m, \delta}}, T_{\varphi_{j}} e^{i j \theta}\right]=0$.

Proof. Under the condition $\delta>\frac{2 \pi}{\arccos \frac{3}{4}}$ there exist $j$ and $l$ satisfying the corresponding condition in the previous theorem. Moreover, $M\left[\varphi_{j}(r) e^{-r^{2}}\right](z)$ satisfies (2.3.1) and (2.3.2). Now, by a similar estimation to that given in Proposition 2.4.5 the assertion follows.

\subsection{Case $0 \leqslant j \leqslant \delta$}

Assume that $\Psi \in S$ has the series-representation $\mathbb{\Psi}$, and let $f_{m, \delta}=r^{m} e^{i \delta \theta}$ where $m \in \mathbb{R}_{+}$and $\delta \in \mathbb{N}$. Under the condition $T_{f_{m, \delta}} T_{\Psi}=T_{\Psi} T_{f_{m, \delta}}$, we aim to find the functions $\Psi_{j}(r)$ whenever $0 \leqslant j \leqslant \delta$. Conversely, we want to determine symbols in $S_{j} \subset S$ such that the corresponding Toeplitz operators commute with $T_{f_{m, \delta}}$.

Proposition 2.6.1. Let $\Psi \in S$ and suppose that $T_{f_{m, \delta}} T_{\Psi}=T_{\Psi} T_{f_{m, \delta}}$. Then for each $j \in \mathbb{N}_{0}$ such that $0 \leqslant j \leqslant \delta$ the functional equation:

$$
M\left[\Psi_{j} e^{-r^{2}}\right](2 z+2 \delta+j+2)=M\left[\Psi_{j} e^{-r^{2}}\right](2 z+j+2) \prod_{l=1}^{j}\left(z+\frac{\delta+m}{2}+l\right) \prod_{l=1}^{\delta-j}(z+j+l)
$$

holds in the half plane $\operatorname{Re}(z)>-\frac{j}{2}-1$.

Proof. Similar to the proof of Proposition 2.4.1.

For each $j$ such that $0 \leqslant j \leqslant \delta$ we consider the meromorphic function:

$$
I_{j}(z):=\prod_{l=1}^{j} \Gamma\left(\frac{z+\frac{\delta+m}{2}+l}{\delta}\right) \prod_{l=1}^{\delta-j} \Gamma\left(\frac{z+j+l}{\delta}\right) \delta^{z} .
$$

We know that

$$
\kappa_{j}(z):=\frac{M\left[\Psi_{j} e^{-r^{2}}\right](2 z+j+2)}{I_{j}(z)}
$$


is holomorphic on the right half plane $\operatorname{Re}(z)>\max \left\{-\frac{j}{2}-1,-\frac{\delta+m}{2}-1\right\}$. By using (2.6.1) together with the functional equation of the Gamma function we conclude that $\kappa_{j}(z)$ is $\delta$-periodic. Therefore, it can be extended to the whole complex plane. Similar to the proof of Proposition 2.4.3 and Theorem 2.4.1, we can show:

Theorem 2.6.1. Let $\Psi \in S$ and suppose that $T_{f_{m, \delta}} T_{\Psi}=T_{\Psi} T_{f_{m, \delta}}$. Then for each $j$ such that $0 \leqslant j \leqslant \delta$ there exists a trigonometric polynomial $\sum_{|l|<\frac{\delta}{4}} a_{l} e^{\frac{2 \pi i l z}{\delta}}$ such that

$$
\Psi_{j}(r)=2 M^{-1}\left[I_{j}(z) \sum_{|l|<\frac{\delta}{4}} a_{l} e^{\frac{2 \pi i l z}{\delta}}\right]\left(r^{2}\right) r^{-j-2} e^{r^{2}}
$$

Now let $f_{m, \delta}$ be a monomial and set $p:=\frac{m+\delta}{2} \in \mathbb{N}$. Then for each $j \in \mathbb{N}_{0}$ such that $0 \leqslant j \leqslant \delta$, one can prove that $I_{j}(z)$ satisfies (1), (2) and (3) in Proposition 2.4.4. Analogous to Corollary 2.4.1 we have:

Corollary 2.6.1. Let $f_{m, \delta}\left(r e^{i \theta}\right)=r^{m} e^{i \delta \theta}$ be a monomial, and let $l \in \mathbb{Z}$ such that $|l|<$ $\frac{\delta}{2 \pi} \arccos \frac{3}{4}$. Then for each $j \in \mathbb{N}_{0}$ such that $0 \leqslant j \leqslant \delta$ the function

$$
\varphi_{j}(r) e^{i j \theta}:=M^{-1}\left[I_{j}(z) e^{\frac{2 \pi i l z}{\delta}}\right]\left(r^{2}\right) r^{-j-2} e^{r^{2}} e^{i j \theta},
$$

defines an element in $\mathcal{E}$ and satisfies $\left[T_{f_{m, \delta}}, T_{\varphi_{j} e^{i j \theta}}\right]=0$. Moreover, $\varphi_{j}(r) e^{i j \theta} \in S$ in the case $l=0$.

We give several examples where there exists one and only one $\Psi_{j} \in S$ (up to multiplication by a constant) such that $\left[T_{f_{m, \delta}}, T_{\Psi_{j} e^{i j \theta}}\right]=0$.

Example 2.6.1. (1): Suppose that $p=n \delta$ for some $n \in \mathbb{N}$, then for each $j$ such that $0<j<$ $\delta$ we have

$$
\begin{aligned}
I_{j}(z) & =\prod_{l=1}^{j} \Gamma\left(\frac{z+l}{\delta}+n\right) \prod_{l=1}^{\delta-j} \Gamma\left(\frac{z+j+l}{\delta}\right) \delta^{z} \\
& =\prod_{l=1}^{j} \prod_{\ell=0}^{n-1}\left(\frac{z+l}{\delta}+\ell\right) \prod_{r=1}^{\delta} \Gamma\left(\frac{z+r}{\delta}\right) \delta^{z} \\
& =(2 \pi)^{\frac{\delta-1}{2}} \delta^{-\frac{1}{2}} \prod_{l=1}^{j} \prod_{\ell=0}^{n-1}\left(\frac{z+l}{\delta}+\ell\right) z \Gamma(z) .
\end{aligned}
$$

(2): $I_{\delta}(z)=\prod_{l=1}^{\delta} \Gamma\left(\frac{z+p+l}{\delta}\right) \delta^{z}=(2 \pi)^{\frac{\delta-1}{2}} \delta^{-p-\frac{1}{2}} \prod_{l=0}^{p}(z+l) \Gamma(z)$.

(3): $I_{0}(z)=(2 \pi)^{\frac{\delta-1}{2}} \delta^{-\frac{1}{2}} z \Gamma(z)$.

Now, applying the same technique as in Corollary 2.4.2, we obtain that there is only one radial symbol $g \in S_{j}$ (up to multiplication by constant) such that $\left[T_{f_{m, \delta}}, T_{g e^{i j \theta}}\right]=0$.

Remark 2.6.1. We would like to mention that Corollary 2.4.1 is valid not only for monomials $f_{m, \delta}$, but also under the weaker condition $p:=\frac{m+\delta}{2} \in \mathbb{N}$. We also note that Corollary 2.6.1 is still valid for all $j \in \mathbb{Z}$ such that $0 \leqslant j \leqslant \min \left\{\delta, \frac{\delta+m}{2}\right\}$ even in the case where $f_{m, \delta}=r^{m} e^{i \delta \theta}$ is not a monomial. 


\subsection{Examples and applications}

In this section, several applications of our results are indicated.

(a) Let $f_{m, \delta}$ be a monomial and suppose that $\left[T_{f_{m, \delta}}, T_{\Psi_{j} e^{i j \theta}}\right]=0$ with $j \in \mathbb{Z}$ such that $j+2\left[\frac{\delta}{4}\right] \geqslant 0$. We would like to investigate the analytic behavior of $\Psi_{j}$ on $\mathbb{R}_{+}$.

For each $j>\delta$ we extend the inverse Mellin transform of $G_{j}(z)$ which is defined on $\mathbb{R}_{+}$ to a holomorphic function on the right half plane.

Fix $\sigma>0$ and define the function $\Lambda_{j}(x)$ for $x \in \mathbb{C}$ with $\operatorname{Re}(x)>0$ by:

$$
\Lambda_{j}(x)=M^{-1}\left[G_{j}(z)\right](x):=\frac{1}{2 \pi i} \int_{\sigma-i \infty}^{\sigma+i \infty} x^{-z} G_{j}(z) d z .
$$

Note that the function $\Lambda_{j}$ is well defined and holomorphic. Indeed, fix $x \in \mathbb{C}$ with $\operatorname{Re}(x)>0$ and write $x=r e^{i \theta}$ where $r=|x|$ and $|\theta|<\frac{\pi}{2}$. Following the proof of Proposition 2.4.4, (2) we obtain

$$
\begin{gathered}
\left|\frac{1}{2 \pi i} \int_{\sigma-i \infty}^{\sigma+i \infty} x^{-z} G_{j}(z) d z\right|=\left|\frac{1}{2 \pi i} \int_{\sigma-i \infty}^{\sigma+i \infty} r^{-z} G_{j}(z) e^{-i z \theta} d z\right| \\
\leqslant \frac{B}{2 \pi} r^{-\sigma} e^{-\sigma} \int_{-\infty}^{\infty}\left(\sigma^{2}+t^{2}\right)^{\frac{\sigma+s}{2}} e^{-|t| \cdot \frac{\pi}{2}+t \theta} d t<\infty
\end{gathered}
$$

where $z=\sigma+i t, B$ and $s$ are real numbers independent of $z$. One can easily check that $\Lambda_{j}$ fulfills the Cauchy-Riemann equations and therefore it defines a holomorphic function on the right half plane. Note that for all $\lambda \in \mathbb{R}_{+}$we have

$$
G_{j}(z) \lambda^{-z}=\lambda^{-z} \int_{\mathbb{R}_{+}} \Lambda_{j}(x) x^{z-1} d x=\int_{\mathbb{R}_{+}} \Lambda_{j}(x)\left(\lambda^{-1} x\right)^{z} \frac{d x}{x}=\int_{\mathbb{R}_{+}} \Lambda_{j}(\lambda x) x^{z-1} d x .
$$

Therefore, the relation

$$
\Lambda_{j}(\lambda x)=M^{-1}\left[G_{j}(z) \lambda^{-z}\right](x)
$$

holds for all $\lambda \in \mathbb{C}$ with $\operatorname{Re}(\lambda)>0$ and all $x \in \mathbb{C}$ such that $\operatorname{Re}(\lambda x)>0$. In particular, for $\lambda=e^{i \theta} \in \mathbb{S}^{1}$ with $-\frac{\pi}{2}<\theta<\frac{\pi}{2}$, (2.7.1) holds in the half space $\arg (x) \in\left(-\frac{\pi}{2}-\right.$ $\left.\theta, \frac{\pi}{2}-\theta\right)$. Therefore, the inverse Mellin transform $M^{-1}\left[G_{j}(z) \lambda^{-z}\right]\left(r^{2}\right)$ is the restriction of a holomorphic function defined in the neighborhood $\arg (x) \in\left(-\frac{\pi}{4}-\frac{\theta}{2}, \frac{\pi}{4}-\frac{\theta}{2}\right)$ of $\mathbb{R}_{+}$.

Now, for each $l \in \mathbb{Z}$ such that $|l|<\frac{\delta}{4}$ we put $\lambda_{l}=e^{-\frac{2 \pi i l}{\delta}}$ and

$$
\Psi_{j, \lambda_{l}}(r)=M^{-1}\left[G_{j}(z) \lambda_{l}^{-z}\right]\left(r^{2}\right) r^{-j-2} e^{r^{2}} .
$$

According to Theorem 2.4.1 and using the notations there we can write

$$
\Psi_{j}(r)=\sum_{|l|<\frac{\delta}{4}} a_{l} \Psi_{j, \lambda_{l}}(r), \quad \text { with } \quad a_{l} \in \mathbb{C} .
$$


Since each $\Psi_{j, \lambda_{l}}(r)$ can be extended to a holomorphic map on $\left(-\frac{\pi}{4}-\frac{\pi l}{\delta}, \frac{\pi}{4}-\frac{\pi l}{\delta}\right)$, we conclude that $\Psi_{j}$ can be extended holomorphically on a neighborhood of $\mathbb{R}_{+}$. Moreover, we have

$$
\begin{aligned}
\Psi_{j, \lambda_{l}}(r)=\Lambda_{j}\left(\lambda_{l} r^{2}\right) r^{-j-2} e^{r^{2}} & =M^{-1}\left[G_{j}(z)\right]\left(\lambda_{l} r^{2}\right) r^{-j-2} e^{r^{2}} \\
& =\Psi_{j, \lambda_{0}}\left(\sqrt{\lambda_{l}} r\right) \lambda_{l}^{\frac{j+2}{2}} e^{\left(1-\lambda_{l}\right) r^{2}}
\end{aligned}
$$

where $\sqrt{\lambda_{l}}:=\exp \left(\frac{1}{2} \log \lambda_{l}\right)$.

Note that, since $H_{j}(z)$ and $I_{j}(z)$ have a growth similar to that of $G_{j}(z)$ we can apply the above arguments also in the cases $j<0$ and $0 \leqslant j \leqslant \delta$.

Corollary 2.7.1. Let $f_{m, \delta}$ be a monomial and let $\Psi \in S$ such that $\left[T_{f_{m, \delta}}, T_{\Psi}\right]=0$. Then for each $j \in \mathbb{Z}, \Psi_{j}$ can be extended to a holomorphic function on a neighborhood of $\mathbb{R}_{+}$. In particular for $j \neq 0, \Psi_{j}$ is non-constant on any interval unless it is the zero function.

Proof. By Theorem 2.5.1 we can assume that $j+2\left[\frac{\delta}{4}\right] \geqslant 0$. Now, suppose that $\Psi_{j}$ is constant on some interval. By the above argument we know that $\Psi_{j}$ can be extended to a holomorphic function on a neighborhood of $\mathbb{R}_{+}$. Therefore, $\Psi_{j}$ is constant on $\mathbb{R}_{+}$. However, by Propositions 2.4.4 (3) and 2.5.3 (3) we have $\Psi_{j}(r)=o\left(r^{|j|-\epsilon}\right)$ as $r \longrightarrow 0$ for all $\epsilon>0$. Hence $\Psi_{j}=0$.

Note that by Example 2.6.1, (3) we have $\Psi_{0}(r)$ is a constant function.

(b) In [17] Theorem $\mathrm{B}$, it was shown that for $u, v \in S$ such that $u$ is radial and non constant the Toeplitz operators $T_{u}$ and $T_{v}$ commute if and only if $v$ is radial. A counterexample was established in the case of a symbol of exponential growth at infinity. In particular, the operators with symbols $u\left(r e^{i \theta}\right)=e^{\left(1-e^{-i \frac{\pi}{4}}\right) r^{2}}$ and $v\left(r e^{i \theta}\right)=e^{i 8 \theta}$ commute. By our results we are able to obtain such examples whenever we fix $v=f_{0, \delta}$ with $\delta>\frac{2 \pi}{\arccos \frac{3}{4}}$.

Example 2.7.1. Let $\delta \in \mathbb{N}$ such that $\delta>\frac{2 \pi}{\arccos \frac{3}{4}}$. According to Corollary 2.6.1 and Remark 2.6.1 we know that for any $l \in \mathbb{Z}$ such that $0<|l|<\frac{\delta}{2 \pi} \arccos \frac{3}{4}$, the operator $T_{f_{m, \delta}}$ commutes with $T_{u_{l, \delta}}$ where $u_{l, \delta}$ is the non-constant radial function:

$$
\begin{aligned}
u_{l, \delta}\left(r e^{i \theta}\right)=\varphi_{0}(r) & =M^{-1}\left[I_{0}(z) e^{\frac{2 \pi i l z}{\delta}}\right]\left(r^{2}\right) r^{-2} e^{r^{2}} \\
& =c_{l, \delta} r^{2} e^{-r^{2} e^{-\frac{2 \pi i l}{\delta}}} r^{-2} e^{r^{2}} \\
& =c_{l, \delta} e^{\left(1-e^{-\frac{2 \pi i l}{\delta}}\right) r^{2}} \in \mathcal{E} .
\end{aligned}
$$

Here $c_{l, \delta}$ is a constant depending on $l$ and $\delta$. In particular, this true when $m=0$, so that the radial symbol $u_{l, \delta}$ commutes with a non-radial bounded symbol $f_{0, \delta}$. Another 
approach to prove that $T_{u_{l, \delta}}$ and $T_{f_{m, \delta}}$ commute is to introduce the following unitary composition operator $U_{l}$ on $H^{2}$ :

$$
U_{l}: H^{2} \longrightarrow H^{2}: f \longrightarrow f\left(e^{-\frac{2 \pi i l}{\delta}} z\right) .
$$

It is shown in [63] that $T_{u_{l, \delta}}=e^{\frac{2 \pi i l}{\delta}} U_{l}$. Moreover, we note that for any symbol $f \in \mathcal{E}$ we have

$$
U_{-l} T_{f} U_{l}=T_{U_{-l} f} \quad \text { on a dense domain } D \subset H^{2} .
$$

In the case $f=f_{m, \delta}$ we have $U_{-l} f=f$, hence

$$
T_{u_{l, \delta}} T_{f_{m, \delta}}=e^{\frac{2 \pi i l}{\delta}} U_{l} U_{-l} T_{f_{m, \delta}} U_{l}=T_{f_{m, \delta}} T_{u_{l, \delta}} .
$$

(c) In [129] I. Louhichi and N.V. Rao conjectured the following: If two Toeplitz operators with bounded symbols acting on the Bergman space over the unit disc commute with a third one, none of them being the identity, then they commute with each other. In [172], N. Vasilevski gave a counterexample in the case of Toeplitz operators acting on the Bergman space over the unit ball $\mathbb{B}^{n}$ with $n>1$.

The next two examples show that a corresponding conjecture is wrong for Toeplitz operators on the Segal-Bargmann space. However, in our counterexamples at least one of the symbols is an unbounded function.

Example 2.7.2. Fix a monomial $f_{m, \delta}$ with $\delta>\frac{2 \pi}{\arccos \frac{3}{4}}$, and let $f_{n, \delta}$ be a monomial such that $m \neq n$. According to Example 2.7.1, there is $u_{1} \in \mathcal{E}$ such that

$$
\left[T_{u_{1}}, T_{f_{m, \delta}}\right] \equiv 0 \equiv\left[T_{u_{1}}, T_{f_{n, \delta}}\right] .
$$

However, $T_{f_{m, \delta}}$ and $T_{f_{n, \delta}}$ do not commute as operators on $\mathbb{P}[z]$. In fact, suppose the contrary then according to condition (2.3.1) we have

$$
M\left[r^{n} e^{-r^{2}}\right](2 k+2 \delta+\delta+2)=M\left[r^{n} e^{-r^{2}}\right](2 k+\delta+2) \prod_{l=1}^{\delta}\left(k+\frac{\delta+m}{2}+l\right),
$$

i.e.

$$
\prod_{l=1}^{\delta}\left(k+\frac{\delta+n}{2}+l\right)=\prod_{l=1}^{\delta}\left(k+\frac{\delta+m}{2}+l\right)
$$

The relation holds if and only if $m=n$.

Example 2.7.3. Let $\delta_{0}$ be an integer such that $\delta_{0}>\frac{2 \pi}{\arccos \frac{3}{4}}$ and put $\delta=2 \delta_{0}$. According to Example 2.7.1 the operators $T_{f_{0, \delta_{0}}}$ and $T_{f_{0, \delta}}$ commute with $T_{u_{1}}$ where $u_{1}=u_{1}\left(r e^{i \theta}\right)=$

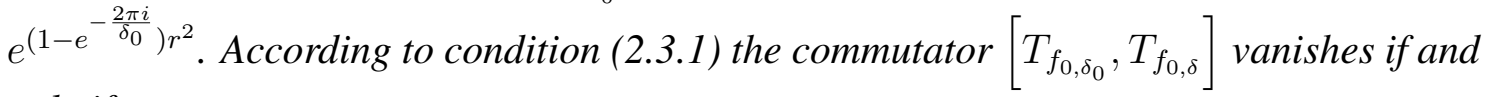
only if

$\frac{\Gamma\left(k+1+\frac{\delta_{0}}{2}\right)}{\Gamma\left(k+2 \delta_{0}+1+\frac{\delta_{0}}{2}\right)} M\left[e^{-r^{2}}\right]\left(2 k+2 \delta_{0}+2 \delta_{0}+2\right)=\frac{\Gamma\left(k+\delta_{0}+1\right)}{\Gamma\left(k+2 \delta_{0}+1\right)} M\left[e^{-r^{2}}\right]\left(2 k+2 \delta_{0}+2\right)$, 
which is equivalent to

$$
\frac{\prod_{l=1}^{\delta_{0}}\left(k+\delta_{0}+l\right)}{\prod_{l=1}^{2 \delta_{0}}\left(k+\frac{\delta_{0}}{2}+l\right)} \prod_{l=1}^{\delta_{0}}\left(k+\delta_{0}+l\right)=1
$$

and would imply that $\prod_{l=1}^{\delta_{0}}\left(z+\delta_{0}+l\right)^{2}=\prod_{l=1}^{2 \delta_{0}}\left(z+\frac{\delta_{0}}{2}+l\right)$ for all $z \in \mathbb{C}$. However, this cannot be true since $z=-\frac{5 \delta_{0}}{2}$ is a root of the right hand side and not a root of the left hand side. Therefore (2.7.3) cannot hold for all $k \in \mathbb{N}_{0}$.

In the case of the Bergman space over the unit disc $\mathbb{D}$ considered in Theorem A0, Z. Cučković and N.V. Rao proved, in [66], the following: Let $z^{l} \bar{z}^{k}$ be a monomial on $\mathbb{D}$ and let $j \in \mathbb{Z}$. Then there is at most one function $\Psi_{j} \in L^{\infty}\left(\mathbb{R}_{+}\right)$such that $\left[T_{z^{l} \bar{z}^{k}}, T_{\Psi_{j} e^{i j \theta}}\right]=0$ (c.f. Theorem A0).

In the case of the Segal-Bargmann space we know that if $\delta<4$ then $\Psi_{j}$ is unique with the above property among the functions of polynomial growth at infinity. Moreover, in Corollary 2.4.2 and Example 2.6.1 we proved for an infinite number of indices $j \in \mathbb{Z}$ the uniqueness of $\Psi_{j}$ as well. So one may conjecture the following:

Conjecture. Let $f_{m, \delta}$ be a fixed monomial. Then for each $j \in \mathbb{Z}$, there is at most one function $g(r)$ defined on the positive real line and of polynomial growth such that $\left[T_{f_{m, \delta}}, T_{g e^{i j \theta}}\right]=0$ on the holomorphic polynomials.

Conjecture. Let $f_{m, \delta}$ be a fixed monomial. Then for each $j \in \mathbb{Z} \backslash\{0\}$, there is no bounded non-zero function $g(r)$ such that $\left[T_{f_{m, \delta}}, T_{g e} e^{i j \theta}\right]=0$ on the holomorphic polynomials. 


\section{Chapter 3}

\section{Commutative algebras of Toeplitz operators on the Segal-Bargmann space}

We construct two types of commutative algebras generated by Toeplitz operators acting on the Segal-Bargmann space $\mathcal{H}_{s}^{2}\left(\mathbb{C}^{n}\right)$. The first is a Banach algebra and refers to bounded symbols in the class $\mathcal{R}_{k}^{n}(h)$ (or simply denoted by $\mathcal{R}_{k}(h)$ ) which is a subspace of $k$-quasi-homogeneous symbols (here $k \in \mathbb{N}^{m}$ with $|k|=n$ ). The second is a $C^{\star}$-algebra and is generated by symbols of the form $\Psi(z)=\Psi(x, y)=a(A(x)) e^{i u y}$ where $z=x+i y \in \mathbb{C}^{n}, A$ is an endomorphism of $\mathbb{R}^{n}$ with $\operatorname{ker} A=\mathcal{H} \subseteq \mathbb{R}^{n}$ is fixed, $u \in \mathcal{H}$ and $a \in L^{\infty}\left(\mathbb{R}^{n}\right)$ are arbitrary. In this case, the result is generalized from the Segal-Bargmann space to all true- $k$-Fock spaces (c.f. Section 3.4 for the definition). Finally, and in the case of the Segal-Bargmann space we are able to combine the two situations forming a more general type of commutative Banach algebras of Toeplitz operators. Roughly speaking, for each fixed $l \in\{0,1, \cdots, n\}, k \in \mathbb{N}_{0}^{m}$ with $|k|=l$ and any linear subspace $\mathcal{H} \subseteq \mathbb{R}^{n-l}$ the Banach algebra generated by the Toeplitz operators

$$
\left\{\mathcal{T}_{\varphi \xi \bar{\xi}^{q}}^{s} a(A(x)) e^{i u \cdot y} \mid \varphi \xi^{p} \bar{\xi}^{q} \in \mathcal{R}_{k}^{l}(h), a \in L^{\infty}\left(\mathbb{R}^{n-l}\right), u \in \mathcal{H}, A \in \mathcal{L}\left(\mathbb{R}^{n-l}\right) \text { s.t. } \operatorname{ker} A=\mathcal{H}\right\}
$$

is commutative on each Segal-Bargmann space $\mathcal{H}_{s}^{2}\left(\mathbb{C}^{n}\right)$ when $s>0$. In the case of $l=n$ (respectively $l=0$ ) we recover the commutative Banach algebra (respectively $C^{\star}$-algebra) of the first (respectively the second) type mentioned above.

\subsection{Introduction}

Commutative Toeplitz algebras acting on the standard weighted Bergman space $A_{\lambda}^{2}\left(\mathbb{B}^{n}\right)$ of the unit ball $\mathbb{B}^{n} \subset \mathbb{C}^{n}$ have been widely studied by several authors [93-97, 144, 145, 168-172]. In [122], and in the case of $n=1$ it was noted that Toeplitz operators with radial symbols are diagonal with respect to the standard orthonormal basis of $A_{\lambda}^{2}(\mathbb{D})$ (c.f. Section 1.4). Hence, the $C^{\star}$-algebra generated by Toeplitz operators of bounded radial symbols is obviously commutative on each weighted Bergman space $A_{\lambda}^{2}(\mathbb{D})$ with $\lambda>-1$. This space of symbols is actually the space of bounded measurable functions which are invariant under the group action 


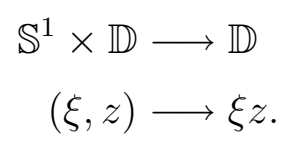

This was the first example which shows that the bounded symbols which are invariant under the group action of a maximal commutative subgroup of the automorphisms of the unit disc (in this case it is the group of rotations) generates commutative $C^{\star}$-algebras of Toeplitz operators on each weighted Bergman space. Later on in [144], R. Quiroga-Barranco and N. Vasilevski gave a general way for constructing commutative $C^{\star}$-algebras of Toeplitz operators from a maximal commutative subgroup of the automorphisms of $\mathbb{B}^{n}$. Namely, for any maximal commutative subgroup in $A u t\left(\mathbb{B}^{n}\right)$ the $C^{\star}$-algebras generated by Toeplitz operators with measurable bounded symbols which are invariant under the action of this subgroup is commutative on each weighted Bergman space $A_{\lambda}^{2}\left(\mathbb{B}^{n}\right)$ of the unit ball. It was noted that there are five different classes of such subgroups: quasi-elliptic, quasi-parabolic, quasi-hyperbolic, nilpotent, and quasi-nilpotent. These classes give rise for $n+2$ different cases of commutative $C^{\star}$-algebra generated by Toeplitz operator acting on the weighted Bergman spaces $A_{\lambda}^{2}\left(\mathbb{B}^{n}\right)$. In the case of the unit disc, $n=1$, and assuming some technical conditions on the ,richness" of the symbol classes it was shown in [93] that any $C^{\star}$-algebra generated by Toeplitz operators is commutative on each $A_{\lambda}^{2}(\mathbb{D})$ if and only if the symbols of the corresponding Toeplitz operators are invariant under the group action of a maximal commutative subgroup in $A u t(\mathbb{D})$.

In the case of the Segal-Bargmann space it remained open how to describe the symbol classes of bounded functions whose corresponding Toeplitz operators generate commutative $C^{\star}$-algebras. However, examples of (non-commutative) $C^{\star}$-algebras generated by Toeplitz operators have been studied in [33]. In this chapter, we aim to give examples of commutative algebras generated by Toeplitz operators acting on each Segal-Bargmann space. Analogous to the case of the unit ball and following the ideas in [172], we show that the Banach algebra generated by Toeplitz operators whose symbols are in a suitable subclass of the $k$-quasihomogeneous functions is commutative on each Segal-Bargmann space $\mathcal{H}_{s}^{2}\left(\mathbb{C}^{n}\right)$. We also construct a commutative $C^{\star}$-algebra generated by the true- $k$-Toeplitz operators whose symbols depend on $x=\operatorname{Re}(z)$ and $y=\operatorname{Im}(z)$ in a suitable way (c.f. Section 3.4).

In the study of Toeplitz operators with $k$-quasi-homogeneous symbols, it is interesting for us to investigate the case where the symbols are only $k$-quasi-radial but unbounded in general. In this situation, we employ a natural extension of the usual notion of Toeplitz operators similar to the one in $[16,117,118]$. Analogous to the case $n=s=1$ in [95], it turns out that any suitable diagonal operator on $\left\{z^{\alpha}, \alpha \in \mathbb{N}_{0}^{n}\right\}$, is a Toeplitz operator with radial symbol in this general notion for any $n \in \mathbb{N}$ and any $s>0$. Consequently, the $C^{\star}$-algebra generated by such operators is commutative on each Segal-Bargmann space $\mathcal{H}_{s}^{2}\left(\mathbb{C}^{n}\right)$ and contains only Toeplitz operators with radial symbols.

Let us now setup the notation used in this chapter and describe explicitly the steps followed in our proofs. 
For each $s>0$ we denote by $\mathcal{H}_{s}^{2}:=\mathcal{H}_{s}^{2}\left(\mathbb{C}^{n}\right)$ the Segal-Bargmann space of entire functions over $\mathbb{C}^{n}$ square integrable with respect to the Gaussian measure

$$
d \mu_{(s)}(z):=d \mu_{\frac{1}{4 s}}(z)=\left(\frac{s}{\pi}\right)^{n} e^{-s|z|^{2}} d v(z) .
$$

From Section 1.3 we know that $\mathcal{H}_{s}^{2}$ is a closed subspace of $L^{2}\left(\mathbb{C}^{n}, d \mu_{(s)}\right)$ with the reproducing kernel

$$
K^{(s)}(z, w)=e^{s z \cdot \bar{w}}
$$

and having the set $\left\{e_{\alpha}^{(s)}(z):=e_{\alpha}^{\frac{1}{4 s}}(z)=\sqrt{\frac{s^{|\alpha|}}{\alpha !}} z^{\alpha}\right\}_{\alpha \in \mathbb{N}_{0}^{n}}$ as an orthonormal basis . For a measurable function $g \in \mathcal{E}_{\frac{1}{4 s}}$ (c.f. Definition 1.3.1) the Toeplitz operator $\mathcal{T}_{g}^{s}$ is the densely defined operator on $\mathcal{H}_{s}^{2}$ and given by

$$
\left(\mathcal{T}_{g}^{s} f\right)(z)=\int g(w) f(w) e^{s z \cdot \bar{w}} d \mu_{(s)}(w)
$$

For each tuple $k=\left(k_{1}, \cdots, k_{m}\right)$ of positive integers such that $k_{1}+\cdots+k_{m}=n$ we write $\mathbb{C}^{n}=\mathbb{C}^{k_{1}} \times \cdots \times \mathbb{C}^{k_{m}}$ and we use the notation

$$
\begin{aligned}
\mathbb{C}^{n} \ni z=\left(z_{1}, \cdots, z_{n}\right) & =\left(\left(z_{1}, \cdots, z_{k_{1}}\right),\left(z_{k_{1}+1}, \cdots, z_{k_{1}+k_{2}}\right), \cdots,\left(z_{n-k_{m}+1}, \cdots, z_{n}\right)\right) \\
& =\left(z_{(1)}, \cdots, z_{(m)}\right) \in \mathbb{C}^{k_{1}} \times \cdots \times \mathbb{C}^{k_{m}}
\end{aligned}
$$

For $j=1, \cdots, m$ we represent each $z_{(j)}=\left(z_{1+\sum_{i<j} k_{i}}, \cdots, z_{\sum_{i \leq j} k_{i}}\right) \in \mathbb{C}^{k_{j}}$ in polar coordinates:

$$
z_{(j)}=r_{j} \xi_{(j)}, \quad \text { with } r_{j}=\left|z_{(j)}\right| \quad \text { and } \xi_{(j)} \in \mathbb{S}^{2 k_{j}-1} \subset \mathbb{C}^{k_{j}} \text {. }
$$

We start by introducing the notion of $k$-quasi-radial and $k$-quasi-homogeneous functions on $\mathbb{C}^{n}$. Let us consider the following action of the product of the spheres $\mathbb{S}^{2 k_{1}-1} \times \cdots \times \mathbb{S}^{2 k_{m}-1}$ on $\mathbb{C}^{n}$

$$
\begin{aligned}
\mathbb{C}^{n} & =\mathbb{C}^{k_{1}} \times \cdots \times \mathbb{C}^{k_{m}} \longrightarrow \mathbb{C}^{n} \\
& z=\left(z_{(1)}, \cdots, z_{(m)}\right) \longrightarrow\left(\xi_{(1)} z_{(1)}, \cdots, \xi_{(m)} z_{(m)}\right),
\end{aligned}
$$

for each $\left(\xi_{(1)}, \cdots, \xi_{(m)}\right) \in \mathbb{S}^{2 k_{1}-1} \times \cdots \times \mathbb{S}^{2 k_{m}-1}$.

A measurable function $\varphi$ defined a.e. on $\mathbb{C}^{n}$ is called $k$-quasi-radial if it is invariant under the above action. Equivalently, a measurable function $\varphi$ is $k$-quasi-radial if it depends only on the radial components $r_{1}, \cdots, r_{m}$ i.e. $\varphi(z)=\varphi\left(r_{1}, \cdots, r_{m}\right)$. The space of all $k$-quasi-radial functions will be denoted by $\mathcal{R}_{k}$. It is clear that for any tuple $k$ we have $\mathcal{R}_{(n)} \subseteq \mathcal{R}_{k} \subseteq \mathcal{R}_{(1, \cdots, 1)}$, i.e. the space of radial function $\mathcal{R}_{(n)}$ (respectively the space of separately radial) is the minimal (respectively the maximal) among these spaces.

For a $k$-quasi-radial function $\varphi$ with a high growth at infinity the Toeplitz operator $\mathcal{T}_{\varphi}^{s}$ may not be defined on the holomorphic polynomials $\mathbb{P}[z]$ (for example when $\varphi \notin L^{2}\left(\mathbb{C}^{n}, d \mu_{(s)}\right)$ ). However, in some cases we may still define a ,,Toeplitz operator" on $\mathbb{P}[z]$ with such symbols extending (3.1.1) (c.f. [16, 117, 118]). In Section 3.2, we consider a more general notion of Toeplitz operators $\tilde{\mathcal{T}}_{\varphi}^{s}$ acting on $\mathbb{P}[z]$ whenever $\varphi$ is in a suitable subclass $L_{k}^{s} \subset \mathcal{R}_{k}$ containing 
the $\mu_{(s)}$-square integrable $k$-quasi-radial functions. Since every function in $\mathcal{H}_{s}^{2}$ can be approximated by holomorphic polynomials it is therefore possible to extend the domain of definition of $\tilde{\mathcal{T}}_{\varphi}^{s}$ according to the behavior of the operator on $\mathbb{P}[z]$. For example, if $\tilde{\mathcal{T}}_{\varphi}^{s}$ is bounded on $\mathbb{P}[z]$ then it can be uniquely extended to a bounded operator on $\mathcal{H}_{s}^{2}$. In this case we do not distinguish between $\tilde{\mathcal{T}}_{\varphi}^{s}$ and its unique bounded extension $\tilde{\mathcal{T}}_{\varphi}^{s} \in \mathcal{L}\left(\mathcal{H}_{s}^{2}\right)$.

By studying suitable Stieltjes moment problems we will show that any operator $T$ on $\mathbb{P}[z]$ such that $T z^{\alpha}=\delta(\alpha) z^{\alpha}$ with $\delta\left(\alpha_{1}\right)=\delta\left(\alpha_{2}\right)$ whenever $\left|\alpha_{1}\right|=\left|\alpha_{2}\right|$ is originated from a Toeplitz operator in the above sense. That is there is a radial function (not unique) $\varphi(r)$ such that $\tilde{\mathcal{T}}_{\varphi(r)}^{s}=T$ on $\mathbb{P}[z]$. Consequently, we verify that the set of operators $\left\{\tilde{\mathcal{T}}_{\varphi}^{s} \in \mathcal{L}\left(\mathcal{H}_{s}^{2}\right), \varphi \in L_{(n)}^{s}\right\}$ is a $C^{\star}$-algebra. Partial results are also obtained for the case of arbitrary $k$-quasi-radial symbols. In particular, the set $\left\{\tilde{\mathcal{T}}_{\varphi}^{s} \in \mathcal{L}\left(\mathcal{H}_{s}^{2}\right), \varphi\left(r_{1}, \cdots, r_{k}\right)=\varphi_{1}\left(r_{1}\right) \cdots \varphi_{k}\left(r_{k}\right) \in L_{k}^{s}\right\}$ is also a $C^{\star}-$ algebra.

In Section 3.3, we consider Toeplitz operators with $k$-quasi-homogeneous symbols which are unbounded in general. More precisely, these symbols are the measurable functions $\psi(z)$ which can be written in the form:

$$
\psi(z)=\varphi\left(r_{1}, \cdots, r_{m}\right) \xi^{p} \bar{\xi}^{q}
$$

for some $\varphi \in \mathcal{R}_{k}$ and some $p, q \in \mathbb{N}_{0}^{n}$ with $p \cdot q=0$. We shall denote by $\mathcal{H}_{k}$ the space of all $k$-quasi-homogeneous functions. For $\psi_{1}, \psi_{2} \in \mathcal{H}_{k} \cap \mathcal{E}_{\frac{1}{4 s}}$, we obtain sufficient conditions that ensure that the Toeplitz operators $\mathcal{T}_{\psi_{1}}^{s}$ and $\mathcal{T}_{\psi_{2}}^{s}$ commute on $\mathbb{P}[z] \subset \mathcal{H}_{s}^{2}$. It turns out that these conditions are independent of the parameter $s$. Following the ideas in [172], we show that for each tuple $k$ there are subspaces $\mathcal{R}_{k}(h) \subset \mathcal{H}_{k}$, parametrized by tuples $h \in \mathbb{N}_{0}^{m}$, containing the bounded $k$-quasi-radial functions such that the Banach algebra generated by Toeplitz operators with symbols in each $\mathcal{R}_{k}(h)$ is commutative. The subspaces $\mathcal{R}_{k}(h)$ are distinct in the case $k \neq(1, \cdots, 1)$. Furthermore, in the case $n \neq 1$ these are not $C^{\star}$-algebras and when $n=1$ they all collapse to the $C^{\star}$-algebra generated by Toeplitz operators with bounded radial symbols.

Section 3.4 is devoted to the construction of a $C^{\star}$-algebra generated by the true- $k$-Toeplitz operators. We shall start the section by defining such operators. In fact, these operators generalize the notion of Toeplitz operators acting on the Segal-Bargmann space to the case of the true- $k$-Fock spaces (c.f. [167]). The symbols we deal with are bounded functions of the form $\Psi(z)=a(A(x)) e^{i u \cdot y}$ where $x, y \in \mathbb{R}^{n}$ with $z=x+i y, A$ is an endomorphism of $\mathbb{R}^{n}$ and $t \in \operatorname{ker} A=\mathcal{H}$. We prove that each true- $k$-Toeplitz operator with such symbol is unitary equivalent to an operator on $L^{2}\left(\mathbb{R}^{n}, d x\right)$ which is a composition of a shift and a multiplication operator. Consequently, for a fixed linear subspace $\mathcal{H} \subseteq \mathbb{R}^{n}$ we show that the $C^{\star}$-algebra generated by true- $k$-Toeplitz operators with symbol of the previously mentioned form is commutative. In particular, this holds true for Toeplitz operators acting on the Segal-Bargmann space. Finally, we are able to combine the two situations considered in Sections 3.3 and 3.4 to form a more general type of commutative Banach-algebra generated by Toeplitz operators of the form (3.0.1). 


\subsection{Commutative $C^{\star}$-algebras of Toeplitz operator}

We start by introducing the Toeplitz operator $\tilde{\mathcal{T}}_{\varphi}^{s}$ on $\mathbb{P}[z]$ with symbol $\varphi$ in a suitable class. Let us consider the following space of measurable functions

$$
L_{k}^{s}:=\left\{\varphi \in \mathcal{R}_{k} \mid \varphi z^{\alpha} \in L^{1}\left(\mathbb{C}^{n}, d \mu_{(s)}\right) \text { for all } \alpha \in \mathbb{N}_{0}^{n}\right\} .
$$

Note that for $\varphi \in L_{k}^{s}$ the complex number $\delta_{\varphi, k, s}(\alpha)$

$$
\delta_{\varphi, k, s}(\alpha):=\left\langle\varphi e_{\alpha}^{(s)}, e_{\alpha}^{(s)}\right\rangle_{(s)}
$$

can be expressed as an integration over $\mathbb{R}^{m}$. Indeed, by (1.3.5) and using integration by polar coordinates $r_{j} \xi_{(j)}$ on each complex space $\mathbb{C}^{k_{j}}$ we obtain

$$
\begin{aligned}
\left\langle\varphi e_{\alpha}^{(s)}, e_{\alpha}^{(s)}\right\rangle_{(s)}= & \frac{s^{n+|\alpha|}}{\pi^{n} \alpha !} \int_{\mathbb{C}^{n}} \varphi(w)\left|w^{\alpha}\right|^{2} e^{-s|w|^{2}} d v(w) \\
= & \frac{s^{n+|\alpha|}}{\alpha ! \pi^{n}} \int_{\mathbb{R}^{m}} \varphi\left(r_{1}, \cdots, r_{m}\right) \prod_{j=1}^{m} r_{j}^{2\left|\alpha_{(j)}\right|+2 k_{j}-1} e^{-s r_{j}^{2}} d r_{j} \\
& \quad \times \int_{\mathbb{S}^{2 k_{j}-1}} \xi_{(j)}^{\alpha_{(j)}} \bar{\xi}_{(j)}^{\alpha_{(j)}} d \sigma\left(\xi_{(j)}\right) \\
= & \frac{2^{m} s^{n+|\alpha|}}{\prod_{j=1}^{m}\left(k_{j}-1+\left|\alpha_{(j)}\right|\right) !} \int_{\mathbb{R}^{m}} \varphi\left(r_{1}, \cdots, r_{m}\right) \prod_{j=1}^{m} r_{j}^{2\left|\alpha_{(j)}\right|+2 k_{j}-1} e^{-s r_{j}^{2}} d r_{j}
\end{aligned}
$$

Remark 3.2.1. The above equation shows that the multi-sequence $\left\{\delta_{\varphi, k, s}(\alpha)\right\}_{\alpha \in \mathbb{N}_{0}^{n}}$ satisfies $\delta_{\varphi, k, s}(\alpha)=\delta_{\varphi, k, s}(\beta)$ whenever $\alpha, \beta \in \mathbb{N}_{0}^{n}$ are such that $\left|\alpha_{(j)}\right|=\left|\beta_{(j)}\right|$ for all $j=1, \cdots, m$. Moreover, if $\varphi \in L_{k}^{s}$ can be written in the form

$$
\varphi\left(r_{1}, \cdots, r_{m}\right)=\prod_{j=1}^{m} \varphi_{j}\left(r_{j}\right)
$$

where $\varphi_{j}$ are functions in $L_{\left(k_{j}\right)}^{s}$ then by Fubini's theorem

$$
\delta_{\varphi, k, s}(\alpha)=\prod_{j=1}^{m} \delta_{\varphi_{j}, k_{j}, s}\left(\alpha_{(j)}\right)=\prod_{j=1}^{m} \delta_{\varphi, k, s}\left(0, \cdots, \alpha_{(j)}, 0, \cdots, 0\right) .
$$

For each $\varphi \in L_{k}^{s}$ we define the Toeplitz operator $\tilde{\mathcal{T}}_{\varphi}^{s}$ on the holomorphic polynomials by

$$
\tilde{\mathcal{T}}_{\varphi}^{s} z^{\alpha}:=\delta_{\varphi, k, s}(\alpha) z^{\alpha}
$$

This notion of Toeplitz operators is a natural extension to the usual definition. Indeed, if $\varphi \in \mathcal{R}_{k}$ is such that $\mathbb{P}[z] \subseteq D\left(\mathcal{T}_{\varphi}^{s}\right):=\left\{f \in \mathcal{H}_{s}^{2} \mid f \varphi \in L^{2}\left(\mathbb{C}^{n}, d \mu_{(s)}\right)\right\}$ then $\tilde{\mathcal{T}}_{\varphi}^{s}=\mathcal{T}_{\varphi}^{s}$ on $\mathbb{P}[z]$. In fact, for each $\alpha \in \mathbb{N}_{0}^{n}$ we have $\varphi z^{\alpha} \in L^{2}\left(\mathbb{C}^{n}, d \mu_{(s)}\right)$ hence by the monotone convergence theorem we can write

$$
\begin{aligned}
{\left[\mathcal{T}_{\varphi}^{s} w^{\alpha}\right](z)=\left\langle\varphi w^{\alpha}, K_{z}^{(s)}\right\rangle_{(s)} } & =\left\langle\varphi w^{\alpha}, \sum_{\beta \in \mathbb{N}_{0}^{n}} e_{\beta}^{(s)}(w) \overline{e_{\beta}^{(s)}(z)}\right\rangle_{(s)}=\sum_{\beta \in \mathbb{N}_{0}^{n}}\left\langle\varphi e_{\alpha}^{(s)}(w), e_{\beta}^{(s)}(w)\right\rangle_{(s)} z^{\beta} \\
& =\left\langle\varphi e_{\alpha}^{(s)}, e_{\alpha}^{(s)}\right\rangle_{(s)} z^{\alpha}=\left[\tilde{\mathcal{T}}_{\varphi}^{s} w^{\alpha}\right](z) .
\end{aligned}
$$


Note that, the operator $\tilde{\mathcal{T}}_{\varphi}^{s}$ uniquely extends to a bounded operator on $\mathcal{H}_{s}^{2}$ if and only if $\left\{\delta_{\varphi, k, s}(\alpha)\right\}_{\alpha \in \mathbb{N}_{0}^{n}} \in l^{\infty}\left(\mathbb{N}_{0}^{n}\right)$. In this case we write $\tilde{\mathcal{T}}_{\varphi}^{s} \in \mathcal{L}\left(\mathcal{H}_{s}^{2}\right)$. Moreover, if $\varphi \in L_{k}^{s}$ is of the form (3.2.3) then by the above remark

$$
\tilde{\mathcal{T}}_{\varphi}^{s} z^{\alpha}=\prod_{j=1}^{m} \tilde{\mathcal{T}}_{\varphi_{j}}^{s} z^{\alpha(j)},
$$

where each $\tilde{\mathcal{T}}_{\varphi_{j}}^{s}$ is the Toeplitz operator defined on the holomorphic polynomials over $\mathbb{C}^{k_{j}}$. Furthermore, if $\varphi \in L_{(n)}^{s}$ then $\tilde{\mathcal{T}}_{\varphi}^{s}$ is a diagonal operator on the monomials and the multisequence of the eigenvalues $\left\{\delta_{\varphi, k, s}(\alpha)\right\}_{\alpha \in \mathbb{N}_{0}^{n}}$ belongs to the space

$$
l_{(n)}\left(\mathbb{N}_{0}^{n}\right):=\left\{\left\{\delta_{\alpha}\right\}_{\alpha \in \mathbb{N}_{0}^{n}} \subset \mathbb{C} \mid \delta_{\alpha}=\delta_{\beta} \text { whenever }|\alpha|=|\beta|\right\} .
$$

Conversely, we will show that for any diagonal operator acting on the monomials by $T z^{\alpha}=$ $\delta_{\alpha} z^{\alpha}$ with $\left\{\delta_{\alpha}\right\}_{\alpha \in \mathbb{N}_{0}^{n}} \in l_{(n)}\left(\mathbb{N}_{0}^{n}\right)$ there is $\varphi \in L_{(n)}^{s}$ (not unique) such that $T=\tilde{\mathcal{T}}_{\varphi}^{s}$ on $\mathbb{P}[z]$. For this reason we have to introduce some results on the so-called Stieltjes moment problem.

Theorem 3.2.1. [71] Let $\left\{\mu_{l}\right\}_{l \in \mathbb{N}_{0}}$ be any sequence of complex numbers. Then there exists a function $g$ in the space

$$
\mathcal{S}^{+}:=\{f \in \mathcal{S}(\mathbb{R}) \mid f(r)=0 \text { for } r<0\} \text {, where } \mathcal{S}(\mathbb{R}) \text { is the Schwartz space over } \mathbb{R}
$$

such that the moments of $g$ satisfies

$$
\int_{0}^{\infty} g(r) r^{l} d r=\mu_{l} \quad \text { for all } l \in \mathbb{N}_{0} .
$$

The following example shows that there exists a non-zero function $g_{0} \in \mathcal{S}^{+}$such that $\int_{0}^{\infty} g_{0}(r) r^{l} d r=0$ for all $l \in \mathbb{N}$. Hence the solutions of (3.2.7) in the space $\mathcal{S}^{+}$are not unique for any complex sequence $\left\{\mu_{l}\right\}_{l \in \mathbb{N}_{0}}$.

Example 3.2.1. [176] Let $g_{0}(r)=e^{-r^{\frac{1}{4}}} \sin \left(r^{\frac{1}{4}}\right)$ then by the change of the variable $r^{\frac{1}{4}}=t$ we have

$$
\begin{aligned}
\int_{0}^{\infty} g_{0}(r) r^{l} d r & =4 \int_{0}^{\infty} e^{-t} t^{4 l+3} \sin (t) d t \\
& =-2 i \int_{0}^{\infty}\left[e^{-(1-i) t}-e^{-(1+i) t}\right] t^{4 l+3} d t \\
& =-2 i(4 l+3) ! 4^{-(l+1)}\left[e^{i \pi(l+1)}-e^{-i \pi(l+1)}\right] \\
& =4^{-l}(4 l+3) ! \sin (l+1) \pi=0
\end{aligned}
$$

More generally, in [71] A. J. Duran characterized the functions $g \in \mathcal{S}^{+}$whose moments (3.2.7) are identically zero. Roughly speaking, if $g \in \mathcal{S}^{+}$then $\int_{0}^{\infty} g(r) r^{l} d r=0$ if and only if there is a function $\phi \in \mathcal{S}^{+}$such that $g(r)=\frac{1}{2} \int_{0}^{\infty} \phi(x) J_{0}(\sqrt{x r}) d x$ where $J_{0}$ is the Bessel function of the first kind. 
Let $\left\{\delta_{\alpha}\right\}_{\alpha \in \mathbb{N}_{0}^{n}} \in l_{(n)}\left(\mathbb{N}_{0}^{n}\right)$ be a fixed sequence of complex numbers and consider the operator $T$ on $\mathbb{P}[z]$ defined by $T z^{\alpha}=\delta_{\alpha} z^{\alpha}$. We aim to find a function $\varphi \in L_{(n)}^{s}$ such that $\tilde{\mathcal{T}}_{\varphi}^{s}=T$ on $\mathbb{P}[z]$. For this reason, we define the sequence $\left\{\mu_{l}\right\}_{l \in \mathbb{N}_{0}}$ by

$$
\mu_{l}:=\delta_{(l, 0, \cdots, 0)} \frac{(n-1+l) !}{2 s^{n+l}} .
$$

According to Theorem 3.2.1 there exists a function $g \in \mathcal{S}^{+}$such that $\mu_{l}=\int_{0}^{\infty} g(r) r^{l} d r$ for all $l \in \mathbb{N}_{0}$. Now let

$$
\varphi(r):=\frac{2}{r^{2(n-1)}} g\left(r^{2}\right) e^{s r^{2}}
$$

be defined on $(0, \infty)$. We claim that $\varphi \in L_{(n)}^{s}$ and $\delta_{\varphi, k, s}(\alpha)=\delta_{\alpha}$ for all $\alpha \in \mathbb{N}_{0}^{n}$. Indeed, since $g$ is in the Schwartz space then $g\left(r^{2}\right) r^{|\alpha|+1} \in \mathcal{S}(0, \infty) \subset L^{1}((0, \infty), d r)$ but

$$
\int_{\mathbb{C}^{n}}|\varphi(z)|\left|z^{\alpha}\right| e^{-s r^{2}} d v(z)=2 \int_{0}^{\infty}\left|g\left(r^{2}\right)\right| r^{|\alpha|+1} d r \int_{\mathbb{S}^{2 n-1}} 1 d \sigma(\xi)<\infty .
$$

Moreover, using (3.2.2) and by the change of variables $u=r^{2}$ we obtain

$$
\begin{aligned}
\tilde{\mathcal{T}}_{\varphi}^{s} z^{(l, 0, \cdots, 0)}=\delta_{\varphi, k, s}((l, 0, \cdots, 0)) z^{(l, 0, \cdots, 0)} & =\left[\frac{2 s^{n+l}}{(n-1+l) !} \int_{0}^{\infty} \varphi(r) r^{2 n-1} r^{2 l} e^{-s r^{2}} d r\right] z^{(l, 0, \cdots, 0)} \\
& =\left[\frac{2 s^{n+l}}{(n-1+l) !} \int_{0}^{\infty} g(u) u^{l} d u\right] z^{(l, 0, \cdots, 0)} \\
& =\delta_{(l, 0, \cdots, 0)} z^{(l, 0, \cdots, 0)}=T z^{(l, 0, \cdots, 0)}
\end{aligned}
$$

Furthermore, for any $\alpha \in \mathbb{N}_{0}^{n}$ we know that

$$
\tilde{\mathcal{T}}_{\varphi}^{s} z^{\alpha}=\delta_{\varphi, k, s}(\alpha) z^{\alpha}=\delta_{\varphi, k, s}((|\alpha|, 0, \cdots, 0)) z^{\alpha}=\delta_{(|\alpha|, 0, \cdots, 0)} z^{\alpha}=\delta_{\alpha} z^{\alpha}=T z^{\alpha}
$$

We arrive now to the description of the $C^{\star}$-algebra of the bounded Toeplitz operators with symbols in $L_{(n)}^{s}$.

Corollary 3.2.1. The set

$$
\mathcal{A}_{(n)}^{s}\left(\mathbb{C}^{n}\right):=\left\{\tilde{\mathcal{T}}_{\varphi}^{s} \mid \varphi \in L_{(n)}^{s} \text { such that } \tilde{\mathcal{T}}_{\varphi}^{s} \in \mathcal{L}\left(\mathcal{H}_{s}^{2}\right)\right\}
$$

is a commutative $C^{\star}$-algebra.

Proof. One can easily check that the set

$$
\mathcal{A}\left(\mathbb{C}^{n}\right)=\left\{T \in \mathcal{L}\left(\mathcal{H}_{s}^{2}\right) \mid T z^{\alpha}=\delta_{\alpha} z^{\alpha} \text { where }\left\{\delta_{\alpha}\right\}_{\alpha \in \mathbb{N}_{0}^{n}} \in l_{(n)}\left(\mathbb{N}_{0}^{n}\right)\right\}
$$

is a commutative $C^{\star}$-algebra. But the above calculation shows that $\mathcal{A}\left(\mathbb{C}^{n}\right)=\mathcal{A}_{(n)}^{s}\left(\mathbb{C}^{n}\right)$.

We generalize the above result to the case of bounded Toeplitz operators with symbols of the form (3.2.3). 
Corollary 3.2.2. The set

$$
\mathcal{A}_{k, 0}^{s,}\left(\mathbb{C}^{n}\right):=\left\{\tilde{\mathcal{T}}_{\varphi}^{s} \in \mathcal{L}\left(\mathcal{H}_{s}^{2}\right) \mid \varphi \in L_{k}^{s} \text { s.t. } \varphi\left(r_{1}, \cdots, r_{m}\right)=\prod_{j=1}^{m} \varphi_{j}\left(r_{j}\right) \text { where } \varphi_{j} \in L_{\left(k_{j}\right)}^{s}\right\}
$$

is a commutative $C^{\star}$-algebra.

Proof. Consider the following commutative $C^{\star}$-algebra

$$
\begin{aligned}
& \mathcal{A}_{0}\left(\mathbb{C}^{n}\right)=\left\{T \in \mathcal{L}\left(\mathcal{H}_{s}^{2}\right) \mid T z^{\alpha}=\delta_{\alpha} z^{\alpha} \text { where } \delta_{\alpha}=\prod_{j=1}^{m} \delta_{\left(0, \cdots, \alpha_{(j)}, 0, \cdots, 0\right)}\right. \text { and } \\
& \left.\qquad \delta_{\alpha}=\delta_{\beta} \text { whenever }\left|\alpha_{(j)}\right|=\left|\beta_{(j)}\right| \text { for all } j=1, \cdots, m \text { and all } \alpha, \beta \in \mathbb{N}_{0}^{n}\right\} .
\end{aligned}
$$

On the one hand, Equations (3.2.2) and (3.2.4) shows that $\mathcal{A}_{k, 0}^{s}\left(\mathbb{C}^{n}\right) \subset \mathcal{A}_{0}\left(\mathbb{C}^{n}\right)$. On the other hand, if $T \in \mathcal{A}_{0}\left(\mathbb{C}^{n}\right)$ then for each $j=1 \cdots, m$ the operator $T_{j}$ defined on the holomorphic polynomials over $\mathbb{C}^{k_{j}}$ by

$$
T_{j} z^{\alpha_{j}}:=T z^{\left(0, \cdots, \alpha_{(j)}, 0, \cdots, 0\right)}
$$

is bounded on $\mathcal{H}_{s}^{2}\left(\mathbb{C}^{k_{j}}\right)$. Therefore by the Corollary 3.2.1 there is $\varphi_{j}\left(r_{j}\right) \in L_{\left(k_{j}\right)}^{s}$ such that $\tilde{\mathcal{T}}_{\varphi_{j}}^{s}=T_{j}$ on $\mathcal{H}_{s}^{2}\left(\mathbb{C}^{k_{j}}\right)$. Now let $\varphi\left(r_{1}, \cdots, r_{m}\right)=\prod_{j=1}^{m} \varphi_{j}\left(r_{j}\right)$ then by (3.2.6) we obtain

$$
\tilde{\mathcal{T}}_{\varphi}^{s} z^{\alpha}=\prod_{j=1}^{m} \tilde{\mathcal{T}}_{\varphi_{j}}^{s} z^{\alpha_{(j)}}=\prod_{j=1}^{m} T_{j} z^{\alpha_{j}}=\prod_{j=1}^{m} \delta_{\left(0, \cdots, \alpha_{(j)}, 0, \cdots, 0\right)} z^{\left(0, \cdots, \alpha_{(j)}, 0, \cdots, 0\right)}=\delta_{\alpha} z^{\alpha}=T z^{\alpha} .
$$

Hence $\mathcal{A}_{0}\left(\mathbb{C}^{n}\right) \subset \mathcal{A}_{k, 0}^{s}\left(\mathbb{C}^{n}\right)$ and the corollary follows.

\subsection{Commutative Banach algebras generated by Toeplitz op- erators with $k$-quasi-homogeneous symbols}

Starting from a tuple $k=\left(k_{1}, \cdots, k_{m}\right)$ of positive integers with $|k|=n$ we construct subspaces $\mathcal{R}_{k}(h) \subset \mathcal{H}_{k} \cap L^{\infty}\left(\mathbb{C}^{n}\right)$ parametrized by tuples $h \in \mathbb{N}_{0}^{m}$. For each fixed tuple $h$ we show that the Toeplitz operators with symbols in $\mathcal{R}_{k}(h)$ commute on each Segal-Bargmann space $\mathcal{H}_{s}^{2}\left(\mathbb{C}^{n}\right)$. Consequently, the Banach algebra generated by the Toeplitz operators with symbols in $\mathcal{R}_{k}(h)$ is commutative.

In [172], N. Vasilevski obtained a similar result for the construction of commutative Banach algebras generated by Toeplitz operators with $k$-quasi-homogeneous symbols acting on the Bergman space of the unit ball $A_{\lambda}^{2}\left(\mathbb{B}^{n}\right)$. As it turns out analogously the result stated in [172] holds for Toeplitz operators on $\mathcal{H}_{s}^{2}\left(\mathbb{C}^{n}\right)$.

Let us start by considering a function $g \in \mathcal{E}_{\frac{1}{4 s}}$ (i.e. $|g(z)| \leq d e^{c|z|^{2}}$ for all $z \in \mathbb{C}^{n}$ where $d, c>0$ s.t. $c<\frac{s}{2}$ ). From Section 1.3 we know that the Toeplitz operator $\mathcal{T}_{g}^{s}$ is well defined on $\mathbb{P}[z]$. In the following we consider $\varphi \xi^{p} \bar{\xi}^{q}, \psi \xi^{u} \bar{\xi}^{v} \in \mathcal{H}_{k} \cap \mathcal{E}_{\frac{1}{4 s}}$ with

$$
\left|p_{(j)}\right|=\left|q_{(j)}\right|, \quad\left|u_{(j)}\right|=\left|v_{(j)}\right| \text { for all } j=1, \cdots, m
$$


and apply $\mathcal{T}_{\varphi \xi \bar{\xi}^{q}}^{s}$ and $\mathcal{T}_{\psi \xi^{u} \bar{\xi}^{v}}^{s}$ to the monomials in order to find a class $\mathcal{R}_{k}(h)$ for which Toeplitz operators with symbols $\varphi \xi^{p} \bar{\xi}^{q}, \psi \xi^{u} \bar{\xi}^{v} \in \mathcal{R}_{k}(h)$ commute on $\mathbb{P}[z]$.

By direct calculation, the following lemma shows how Toeplitz operators with $k$-quasihomogeneous symbols behave on $\mathbb{P}[z]$.

Lemma 3.3.1. Let $\varphi \xi^{p} \bar{\xi}^{q} \in \mathcal{H}_{k}$ be a k-quasi-homogeneous function i.e. $\varphi \in \mathcal{R}_{k}$ and $p \cdot q=0$. Moreover, suppose $\varphi \in \mathcal{E}_{\frac{1}{4 s}}$ then for each $\alpha \in \mathbb{N}^{n}$ the Toeplitz operator $\mathcal{T}_{\varphi \xi^{p} \bar{\xi}^{q}}^{s}$ fulfills:

$$
\mathcal{T}_{\varphi \xi^{p} \bar{\xi}^{q}} z^{\alpha}= \begin{cases}\tilde{\delta}_{\varphi, k, p, q, s}(\alpha) z^{\alpha+p-q} & \alpha_{l}+p_{l}-q_{l} \geqslant 0 \text { for all } l=1, \cdots, n \\ 0 & \text { else }\end{cases}
$$

where

$$
\begin{aligned}
\tilde{\delta}_{\varphi, k, p, q, s}(\alpha)= & \frac{2^{m} s^{n+|\alpha+p-q|}(\alpha+p) !}{(\alpha+p-q) ! \prod_{j=1}^{m}\left(k_{j}-1+\left|\alpha_{(j)}+p_{(j)}\right|\right) !} \\
& \times \int_{\mathbb{R}^{m}} \varphi\left(r_{1}, \cdots, r_{m}\right) \prod_{j=1}^{m} r_{j}^{\left|2 \alpha_{(j)}+p_{(j)}-q_{(j)}\right|+2 k_{j}-1} e^{-s r_{j}^{2}} d r_{j} .
\end{aligned}
$$

Proof. Since $\varphi \in \mathcal{E}_{\frac{1}{4 s}}$ then by the monotone convergence theorem we can write

$$
\begin{aligned}
\mathcal{T}_{\varphi \xi^{p} \bar{\xi}^{q}}^{s} z^{\alpha}= & \int_{\mathbb{C}^{n}} \varphi(w) \xi^{p} \bar{\xi}^{q} w^{\alpha} e^{s z \bar{w}}\left(\frac{s}{\pi}\right)^{n} e^{-s|w|^{2}} d v(w) \\
= & \sum_{\beta \in \mathbb{N}^{n}} \frac{z^{\beta} s^{|\beta|+n}}{\beta ! \pi^{n}} \int_{\mathbb{R}^{m}} \varphi\left(r_{1}, \cdots, r_{m}\right) \prod_{j=1}^{m} r_{j}^{\left|\alpha_{(j)}\right|+\left|\beta_{(j)}\right|+2 k_{j}-1} e^{-s r_{j}^{2}} d r_{j} \\
& \times \int_{\mathbb{S}^{2 k_{j}-1}} \xi_{(j)}^{\alpha_{(j)}+p_{(j)}} \frac{\bar{\xi}_{(j)}}{\beta_{(j)}+q_{(j)}} d \sigma\left(\xi_{(j)}\right) .
\end{aligned}
$$

The above terms are all zero except when $\alpha_{(j)}+p_{(j)}=\beta_{(j)}+q_{(j)}$ for all $j=1 \cdots, m$. So that $\beta=\alpha+p-q$ which means $\alpha_{l}+p_{l}-q_{l}$ should be non-negative for all $l=1, \cdots, n$. Using (1.3.5) we obtain

$$
\begin{aligned}
\mathcal{T}_{\varphi \xi^{p} \bar{\xi}^{q}}^{s} z^{\alpha}= & \frac{2^{m} s^{|\alpha+p-q|+n}}{(\alpha+p-q) !} \frac{\prod_{j=1}^{m}\left(\alpha_{(j)}+p_{(j)}\right) !}{\prod_{j=1}^{m}\left(k_{j}-1+\left|\alpha_{(j)}+p_{(j)}\right|\right) !} \\
& \times \int_{\mathbb{R}^{m}} \varphi\left(r_{1}, \cdots, r_{m}\right) \prod_{j=1}^{m} r_{j}^{\left|2 \alpha_{(j)}+p_{(j)}-q_{(j)}\right|+2 k_{j}-1} e^{-s r_{j}^{2}} d r_{j} z^{\alpha+p-q} .
\end{aligned}
$$

Let us now consider two Toeplitz operators one with $k$-quasi-radial symbol and the second with $k$-quasi-homogeneous symbol.

Proposition 3.3.1. Let $k=\left(k_{1}, \cdots, k_{m}\right)$ be a tuple of positive integers with $k_{1}+\cdots+k_{m}=n$ and consider two functions $\varphi_{1}, \varphi_{2} \in \mathcal{R}_{k} \cap \mathcal{E}_{\frac{1}{4 s}}$. Then for any couple of orthogonal multi-indices $p, q \in \mathbb{N}_{0}^{n}$ such that $\left|p_{(j)}\right|=\left|q_{(j)}\right|$ for all $j \stackrel{u^{s}}{=} 1, \cdots, m$ the Toeplitz operators $\mathcal{T}_{\varphi_{1}}^{s}$ and $\mathcal{T}_{\varphi_{2} \xi^{p}}^{s} \bar{\xi}^{q}$ commute on $\mathbb{P}[z]$. 
Proof. Let $\alpha \in \mathbb{N}_{0}^{n}$ be such that $\alpha_{l}+p_{l}-q_{l} \geqslant 0$ for all $l=1, \cdots, n$ then by (3.2.5) and (3.3.1) we have

- $\mathcal{T}_{\varphi_{1}}^{s} \mathcal{T}_{\varphi_{2} \xi^{p}}^{s} \bar{\xi}^{q} z^{\alpha}=\tilde{\delta}_{\varphi_{2}, k, p, q, s}(\alpha) \mathcal{T}_{\varphi_{1}}^{s} z^{\alpha+p-q}=\tilde{\delta}_{\varphi_{2}, k, p, q, s}(\alpha) \delta_{\varphi_{1}, k, s}(\alpha+p-q) z^{\alpha+p-q}$

- $\mathcal{T}_{\varphi_{2} \xi^{p} \bar{\xi}^{q}}^{s} \mathcal{T}_{\varphi_{1}}^{s} z^{\alpha}=\delta_{\varphi_{1}, k, s}(\alpha) \mathcal{T}_{\varphi_{2} \xi^{p} \bar{\xi}^{q}}^{s} z^{\alpha}=\delta_{\varphi_{1}, k, s}(\alpha) \tilde{\delta}_{\varphi_{2}, k, p, q, s}(\alpha) z^{\alpha+p-q}$.

Hence the operators $\mathcal{T}_{\varphi_{1}}^{s}$ and $\mathcal{T}_{\varphi_{2} \xi^{p} \bar{\xi}^{q}}^{s}$ commute on $\mathbb{P}[z]$ if and only if

$$
\tilde{\delta}_{\varphi_{2}, k, p, q, s}(\alpha) \delta_{\varphi_{1}, k, s}(\alpha+p-q)=\delta_{\varphi_{1}, k, s}(\alpha) \tilde{\delta}_{\varphi_{2}, k, p, q, s}(\alpha)
$$

From Remark 3.2.1 we know that $\delta_{\varphi_{1}, k, s}(\alpha)=\delta_{\varphi_{1}, k, s}(\beta)$ whenever $\left|\alpha_{(j)}\right|=\left|\beta_{(j)}\right|$ for all $j=$ $1, \cdots, m$. Since $\left|p_{(j)}\right|=\left|q_{(j)}\right|$ for all $j=1, \cdots, m$ we get $\delta_{\varphi_{1}, k, s}(\alpha)=\delta_{\varphi_{1}, k, s}(\alpha+p-q)$ and (3.3.3) holds.

As a consequence, we obtain the following corollaries

Corollary 3.3.1. For a given tuple $k=\left(k_{1}, \cdots, k_{m}\right)$ let $p, q \in \mathbb{N}_{0}^{n}$ be a pair of orthogonal multi-indicies such that $\left|p_{(j)}\right|=\left|q_{(j)}\right|$ for all $j=1, \cdots, m$. Then for any $\varphi \in \mathcal{R}_{k} \cap \mathcal{E}_{\frac{1}{4 s}}$ we have

$$
\mathcal{T}_{\varphi}^{s} \mathcal{T}_{\xi^{p} \bar{\xi}^{q}}^{s}=\mathcal{T}_{\xi^{p} \bar{\xi}^{q}}^{s} \mathcal{T}_{\varphi}^{s}=\mathcal{T}_{\varphi \xi^{p} \bar{\xi}^{q}}^{s}
$$

where the above equality holds on $\mathbb{P}[z]$.

Proof. First we remark that for any $p, q \in \mathbb{N}_{0}^{n}$ such that $\left|p_{(j)}\right|=\left|q_{(j)}\right|$ for all $j=1, \cdots, m$ and any $\varphi \in \mathcal{R}_{k} \cap \mathcal{E}_{\frac{1}{4 s}}$ the following equality holds

$$
\begin{aligned}
\tilde{\delta}_{\varphi, k, p, q, s}(\alpha)= & \frac{2^{m} s^{n+|\alpha|}(\alpha+p) !}{(\alpha+p-q) ! \prod_{j=1}^{m}\left(k_{j}-1+\left|\alpha_{(j)}+p_{(j)}\right|\right) !} \\
& \times \int_{\mathbb{R}^{m}} \varphi\left(r_{1}, \cdots, r_{m}\right) \prod_{j=1}^{m} r_{j}^{\left|2 \alpha_{(j)}\right|+2 k_{j}-1} e^{-s r_{j}^{2}} d r_{j} \\
= & \delta_{\varphi, k, s}(\alpha) \prod_{j=1}^{m} \frac{\left(k_{j}-1+\left|\alpha_{(j)}\right|\right) !\left(\alpha_{(j)}+p_{(j)}\right) !}{\left(\alpha_{(j)}+p_{(j)}-q_{(j)}\right) !\left(k_{j}-1+\left|\alpha_{(j)}+p_{(j)}\right|\right) !}=\delta_{\varphi, k, s}(\alpha) \eta_{k, p, q}(\alpha) .
\end{aligned}
$$

The first equality of (3.3.4) follows from Proposition 3.3.1. In order to prove the second equality we note that $\delta_{1, k, s}(\alpha)=\left\langle e_{\alpha}^{(s)}, e_{\alpha}^{(s)}\right\rangle_{(s)}=1$. Now by Lemma 3.3.1 we know that for any $\alpha$ such that $\alpha_{l}+p_{l}-q_{l} \geqslant 0$ :

$$
\begin{aligned}
\mathcal{T}_{\varphi \xi^{p} \bar{\xi}^{q}}^{s} z^{\alpha} & =\tilde{\delta}_{\varphi, k, p, q, s}(\alpha) z^{\alpha+p-q}=\delta_{\varphi, k, s}(\alpha) \eta_{k, p, q}(\alpha) z^{\alpha+p-q} \\
& =\delta_{\varphi, k, s}(\alpha) \delta_{1, k, s}(\alpha) \eta_{k, p, q}(\alpha) z^{\alpha+p-q}=\delta_{\varphi, k, s}(\alpha) \tilde{\delta}_{1, k, p, q, s}(\alpha) z^{\alpha+p-q} \\
& =\mathcal{T}_{\xi p \bar{\xi}^{q}}^{s} \delta_{\varphi, k, s}(\alpha) z^{\alpha}=\mathcal{T}_{\xi p \bar{\xi}^{q}}^{s} \mathcal{T}_{\varphi}^{s} z^{\alpha}
\end{aligned}
$$


Corollary 3.3.2. Given a tuple $k=\left(k_{1}, \cdots, k_{m}\right)$ and a pair of orthogonal multi-indicies $p, q \in$ $\mathbb{N}_{0}^{n}$ such that $\left|p_{(j)}\right|=\left|q_{(j)}\right|$ for all $j=1, \cdots, m$, consider

$$
\tilde{p}_{(j)}=\left(0, \cdots, 0, p_{(j)}, 0, \cdots, 0\right) \quad \text { and } \quad \tilde{q}_{(j)}=\left(0, \cdots, 0, q_{(j)}, 0, \cdots, 0\right)
$$

then

$$
\mathcal{T}_{\xi^{\tilde{p}}\left(j_{1}\right) \bar{\xi}^{\tilde{q}_{\left(j_{1}\right)}}}^{s} \mathcal{T}_{\xi^{\tilde{p}\left(j_{2}\right)}}^{s} \bar{\xi}^{\tilde{q}\left(j_{2}\right)}=\mathcal{T}_{\xi^{\tilde{p}}\left(j_{1}\right)}^{s}+\tilde{p}_{\left(j_{2}\right)} \bar{\xi}^{\tilde{q}_{\left(j_{1}\right)}+\tilde{q}_{\left(j_{2}\right)}}
$$

In particular, the operators $\mathcal{T}_{\xi^{\tilde{p}}(j)}^{s} \bar{\xi}^{\tilde{q}(j)}$ mutually commute for $j=1, \cdots$, m and

$$
\prod_{j=1}^{m} \mathcal{T}_{\xi^{\tilde{p}(j)}}^{s} \bar{\xi}^{\tilde{q}_{(j)}}=\mathcal{T}_{\xi^{p} \bar{\xi}^{q}}^{s} .
$$

Proof. It is easy to see that

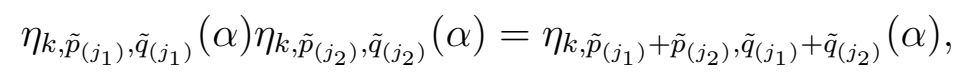

where $\eta_{k, p, q}(\alpha)$ is given by (3.3.5). Therefore

$$
\begin{aligned}
& \mathcal{T}_{\xi^{\tilde{p}\left(j_{1}\right)} \bar{\xi}^{\tilde{q}}\left(j_{1}\right)}^{s} \mathcal{T}_{\xi^{\tilde{p}}\left(j_{2}\right)}^{s} \tilde{\xi}^{\tilde{q}\left(j_{2}\right)} z^{\alpha}=\eta_{k, \tilde{p}_{\left(j_{2}\right)}, \tilde{q}_{\left(j_{2}\right)}}(\alpha) \mathcal{T}_{\xi^{\tilde{p}\left(j_{1}\right)}}^{s} \bar{\xi}^{\tilde{q}_{\left(j_{1}\right)}} z^{\alpha+\tilde{p}_{\left(j_{2}\right)}-\tilde{q}_{\left(j_{2}\right)}} \\
& =\eta_{k, \tilde{p}_{\left(j_{2}\right)}, \tilde{q}_{\left(j_{2}\right)}}(\alpha) \eta_{k, \tilde{p}_{\left(j_{1}\right)}, \tilde{q}_{\left(j_{1}\right)}}(\alpha) z^{\alpha+\tilde{p}_{\left(j_{1}\right)}+\tilde{p}_{\left(j_{2}\right)}-\tilde{q}_{\left(j_{1}\right)}-\tilde{q}_{\left(j_{2}\right)}}
\end{aligned}
$$

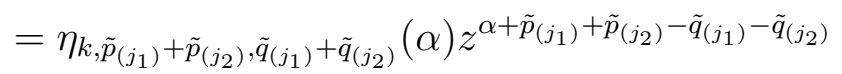

$$
\begin{aligned}
& =\mathcal{T}_{\xi^{\tilde{p}\left(j_{1}\right)}}^{s}+\tilde{p}\left(j_{2}\right) \bar{\xi}^{\tilde{q}\left(j_{1}\right)+\tilde{q}_{\left(j_{2}\right)}} z^{\alpha} .
\end{aligned}
$$

We are ready now to consider two Toeplitz operators with $k$-quasi-homogeneous symbols.

Theorem 3.3.1. Given a tuple $k=\left(k_{1}, \cdots, k_{m}\right)$ of positive integers with $k_{1}+\cdots+k_{m}=n$. Let $p, q, u, v \in \mathbb{N}_{0}^{n}$ such that $p \cdot q=0, u \cdot v=0$ and

$$
\left|p_{(j)}\right|=\left|q_{(j)}\right|, \quad\left|u_{(j)}\right|=\left|v_{(j)}\right| \quad \text { for all } j=1, \cdots, m .
$$

Moreover, suppose that for each $l=1, \cdots, n$ one of the following conditions holds:

- $p_{l}=q_{l}=0$,

- $u_{l}=v_{l}=0$,

- $p_{l}=u_{l}=0$,

- $q_{l}=v_{l}=0$.

Then for any functions $\varphi, \psi \in \mathcal{R}_{k} \cap \mathcal{E}_{\frac{1}{4 s}}$ the Toeplitz operators $\mathcal{T}_{\varphi \xi^{p} \bar{\xi}^{q}}^{s}$ and $\mathcal{T}_{\psi \xi^{u} \bar{\xi}}^{s}$ commute on $\mathbb{P}[z]$.

Proof. Let $\alpha \in \mathbb{N}_{0}^{n}$ be such that $\alpha_{l}+p_{l}-q_{l}+u_{l}-v_{l} \geqslant 0$ for all $l=1, \cdots, n$. Then by (3.3.1) we know that: 
- $\mathcal{T}_{\varphi \xi^{p} \bar{\xi}^{q}}^{s} \mathcal{T}_{\psi \xi^{u} \bar{\xi}}^{s} z^{\alpha}=\tilde{\delta}_{\psi, k, u, v, s}(\alpha) \mathcal{T}_{\varphi \xi^{p} \bar{\xi}^{q}}^{s} z^{\alpha+u-v}=\tilde{\delta}_{\psi, k, u, v, s}(\alpha) \tilde{\delta}_{\varphi, k, p, q, s}(\alpha+u-v) z^{\alpha+u-v+p-q}$,

- $\mathcal{T}_{\psi \xi^{u} \bar{\xi}^{v}}^{s} \mathcal{T}_{\varphi \xi^{p} \bar{\xi}^{q}}^{s} z^{\alpha}=\tilde{\delta}_{\varphi, k, p, q, s}(\alpha) \mathcal{T}_{\psi \xi^{u} \bar{\xi}}^{s} z^{\alpha+p-q}=\tilde{\delta}_{\varphi, k, p, q, s}(\alpha) \tilde{\delta}_{\psi, k, u, v, s}(\alpha+p-q) z^{\alpha+u-v+p-q}$.

Then $\mathcal{T}_{\varphi \xi^{p} \bar{\xi}^{q}}^{s}$ and $\mathcal{T}_{\psi \xi^{u} \bar{\xi}^{v}}^{s}$ commute on $\mathbb{P}[z]$ if and only if for every $\alpha \in \mathbb{N}_{0}^{n}$ with $\alpha_{l}+p_{l}-q_{l}+$ $u_{l}-v_{l} \geqslant 0$ we have

$$
\tilde{\delta}_{\psi, k, u, v, s}(\alpha) \tilde{\delta}_{\varphi, k, p, q, s}(\alpha+u-v)=\tilde{\delta}_{\varphi, k, p, q, s}(\alpha) \tilde{\delta}_{\psi, k, u, v, s}(\alpha+p-q) .
$$

Since $\left|p_{(j)}\right|=\left|q_{(j)}\right|$ and $\left|u_{(j)}\right|=\left|v_{(j)}\right|$ for all $j=1, \cdots, m$ the above equation takes the form

$$
\begin{aligned}
& \frac{2^{m} s^{n+|\alpha|}(\alpha+u) !}{(\alpha+u-v) ! \prod_{j=1}^{m}\left(k_{j}-1+\left|\alpha_{(j)}+u_{(j)}\right|\right) !} \int_{\mathbb{R}^{m}} \psi\left(r_{1}, \cdots, r_{m}\right) \prod_{j=1}^{m} r_{j}^{2\left|\alpha_{(j)}\right|+2 k_{j}-1} e^{-s r^{2}} d r_{j} \\
& \times \frac{2^{m} s^{n+|\alpha|}(\alpha+u-v+p) !}{(\alpha+u-v+p-q) ! \prod_{j=1}^{m}\left(k_{j}-1+\left|\alpha_{(j)}+p_{(j)}\right|\right) !} \\
& \times \int_{\mathbb{R}^{m}} \varphi\left(r_{1}, \cdots, r_{m}\right) \prod_{j=1}^{m} r_{j}^{2\left|\alpha_{(j)}\right|+2 k_{j}-1} e^{-s r^{2}} d r_{j} \\
& =\frac{2^{m} s^{n+|\alpha|}(\alpha+p) !}{(\alpha+p-q) ! \prod_{j=1}^{m}\left(k_{j}-1+\left|\alpha_{(j)}+p_{(j)}\right|\right) !} \int_{\mathbb{R}^{m}} \varphi\left(r_{1}, \cdots, r_{m}\right) \prod_{j=1}^{m} r_{j}^{2\left|\alpha_{(j)}\right|+2 k_{j}-1} e^{-s r^{2}} d r_{j} \\
& \times \frac{2^{m} s^{n+|\alpha|}(\alpha+p-q+u) !}{(\alpha+p-q+u-v) ! \prod_{j=1}^{m}\left(k_{j}-1+\left|\alpha_{(j)}+u_{(j)}\right|\right) !} \\
& \times \int_{\mathbb{R}^{m}} \psi\left(r_{1}, \cdots, r_{m}\right) \prod_{j=1}^{m} r_{j}^{2\left|\alpha_{(j)}\right|+2 k_{j}-1} e^{-s r^{2}} d r_{j} .
\end{aligned}
$$

Therefore the Toeplitz operators $\mathcal{T}_{\varphi \xi^{p} \bar{\xi}^{q}}^{s}$ and $\mathcal{T}_{\psi \xi^{u} \bar{\xi}^{v}}^{s}$ commute on $\mathbb{P}[z]$ if

$$
\frac{(\alpha+u-v+p) !(\alpha+u) !}{(\alpha+u-v) !}=\frac{(\alpha+p-q+u) !(\alpha+p) !}{(\alpha+p-q) !}
$$

The equality holds for all $\alpha \in \mathbb{N}_{0}^{n}$ if for each $l=1, \cdots, n$ one of the above conditions holds.

By the above theorem we are now able to construct commutative Banach algebras generated by Toeplitz operators with $k$-quasi-homogeneous symbols. Let us start by a tuple $k=$ $\left(k_{1}, \cdots, k_{m}\right)$ of positive integers with $k_{1}+\cdots+k_{m}=n$. Since the perturbation of the coordinates is a biholomorphism of $\mathbb{C}^{n}$ and gives an unitary equivalence of the Toeplitz operator (via the biholomorphic map), we can assume $k_{1} \leqslant k_{2} \leqslant \cdots \leqslant k_{m}$.

Now consider a tuple $h=\left(h_{1}, \cdots, h_{m}\right)$ with $h_{j}=0$ if $k_{j}=1$ and $1 \leqslant h_{j} \leqslant k_{j}-1$ and if $k_{j_{0}}=k_{j_{1}}$ with $j_{0}<j_{1}$ put $h_{j_{0}} \leqslant h_{j_{1}}$. Put

$$
p_{(j)}=\left(p_{j, 1}, \cdots, p_{j, h_{j}}, 0 \cdots, 0\right), \quad q_{(j)}=\left(0, \cdots, 0, q_{j, h_{j}+1}, \cdots, q_{j, k_{j}}\right)
$$

where $p_{1,1}, \cdots, p_{m, h_{m}}, q_{1, h_{1}+1}, \cdots, q_{m, k_{m}} \in \mathbb{N}_{0}^{n}$ satisfies

$$
\left|p_{(j)}\right|=\left|q_{(j)}\right|
$$


Now let $\mathcal{R}_{k}(h)$ be the space of all bounded $k$-quasi-homogeneous symbols of the form

$$
\varphi\left(r_{1}, \cdots, r_{m}\right) \xi^{p} \bar{\xi}^{q},
$$

where $p, q$ are multi-indices of the form (3.3.6) and satisfying (3.3.7) then by Theorem (3.3.1) we obtain

Corollary 3.3.3. The Toeplitz operators with symbols in $\mathcal{R}_{k}(h)$ generates a commutative Banach algebra in $\mathcal{L}\left(\mathcal{H}_{s}^{2}\right)$. Moreover, for $n>2$ and $k \neq(1, \cdots, 1)$ these algebras are not $C^{\star}$-algebras.

\subsection{Commutative $C^{\star}$-algebras generated by true- $k$-Toeplitz operators}

In this section, for each multi-index $k=\left(k_{1}, \cdots, k_{n}\right) \in \mathbb{N}^{n}$ we consider the true- $k$-Fock space $F_{(k)}^{2}\left(\mathbb{C}^{n}\right)$ as was defined in [167]. Via the orthogonal projection $P_{(k)}$ from $L^{2}\left(\mathbb{C}^{n}, d \mu:=d \mu_{(1)}\right)$ onto $F_{(k)}^{2}\left(\mathbb{C}^{n}\right)$ we define the true- $k$-Toeplitz operator $T_{\varphi}^{(k)}$, with suitable symbol $\varphi$, on $F_{(k)}^{2}\left(\mathbb{C}^{n}\right)$ as $P_{(k)} M_{\varphi}$ where $M_{\varphi}$ is the multiplication operator by $\varphi$. Consider two bounded functions $\theta$ and $\gamma$ on $\mathbb{C}^{n}$ such that

$$
\theta(z)=\theta(x, y)=a(A(x)) e^{i u \cdot y}, \quad \gamma(z)=\delta(x, y)=b(B(x)) e^{i t \cdot y}
$$

where $z=x+i y, x, y \in \mathbb{R}^{n}$, and $a, b$ are functions on $\mathbb{R}^{n}$. Moreover, $A$ and $B$ are linear maps on $\mathbb{R}^{n}$ and $u, t \in \mathbb{R}^{n}$. We show that the operator $T_{\theta}^{(k)}$ on $F_{(k)}^{2}\left(\mathbb{C}^{n}\right)$ is unitary equivalent to an operator on $L^{2}\left(\mathbb{R}^{n}, d x\right)$ which is a composition of a shift and a multiplication operator. The point here is that the equivalence arise from an isometric isomorphism from $F_{(k)}^{2}\left(\mathbb{C}^{n}\right)$ onto $L^{2}\left(\mathbb{R}^{n}, d x\right)$. This isomorphism was introduced by $\mathrm{N}$. Vasilevski in [167]. As a consequence, we show that the two true- $k$-Toeplitz operator $T_{\theta}^{(k)}$ and $T_{\gamma}^{(k)}$ commute whenever $u \in \operatorname{ker} B$ and $t \in \operatorname{ker} A$. In particular, for any subspace $\mathcal{H} \subset \mathbb{R}^{n}$ the $C^{\star}$-algebra generated by the set

$$
\left\{T_{a(A(x)) e^{i u \cdot y}}^{(k)}, a \in L^{\infty}\left(\mathbb{R}^{n}\right), A \text { is an endomorphism of } \mathbb{R}^{n} \text { with } \operatorname{ker} A=\mathcal{H} \text { and } u \in \mathcal{H}\right\}
$$

is commutative. We would like then to mention that our results still hold true for the case of the Segal-Bargmann space. Finally, we combine the algebras obtained here with those given in the previous section to form a more general type of commutative Banach algebra generated by the operators in (3.0.1).

For $k \in \mathbb{N}$ the $k$-Fock space $F_{k}^{2}(\mathbb{C})$ is defined to be the closure of the set of all smooth functions in $L^{2}(\mathbb{C}, d \mu)$ satisfying the equation

$$
\frac{\partial^{k}}{\partial \bar{z}^{k}} \varphi=0
$$

The true- $k$-Fock space is defined as follows:

$$
\begin{aligned}
& F_{(k)}^{2}(\mathbb{C})=F_{k}^{2}(\mathbb{C}) \ominus F_{k-1}^{2}(\mathbb{C}), \quad \text { for } k>1 \\
& F_{(1)}^{2}(\mathbb{C})=\mathcal{H}_{1}^{2}(\mathbb{C}) .
\end{aligned}
$$


Now for each multi-index $k=\left(k_{1}, \cdots, k_{n}\right) \in \mathbb{N}^{n}$ the true- $k$-Fock space $F_{(k)}^{2}\left(\mathbb{C}^{n}\right)$ on $\mathbb{C}^{n}$ is defined as the tensor product of the true-poly-Fock spaces on $\mathbb{C}$

$$
F_{(k)}^{2}\left(\mathbb{C}^{n}\right):=\bigotimes_{j=1}^{n} F_{\left(k_{j}\right)}^{2}(\mathbb{C}),
$$

with the induced norm from $L^{2}\left(\mathbb{C}^{n}, d \mu\right)$. We denote by $P_{(k)}$ the orthogonal projection from $L^{2}\left(\mathbb{C}^{n}, d \mu\right)$ onto $F_{(k)}^{2}$. Moreover, we introduce on $\mathbb{R}^{n}$ the function

$$
\tilde{h}_{k}(y):=\prod_{j=1}^{n} h_{k_{j}}\left(y_{j}\right),
$$

where $h_{k_{j}}(u)$ is the function defined on $\mathbb{R}$ by

$$
h_{k_{j}}(u)=\left(2^{k_{j}} k_{j} ! \sqrt{\pi}\right)^{-\frac{1}{2}} H_{k_{j}}(u) e^{-\frac{u^{2}}{2}}
$$

and $H_{k_{j}}(u):=(-1)^{k_{j}} e^{u^{2}} \frac{d^{k_{j}}}{d u^{k_{j}}} e^{-u^{2}}$ is the Hermite polynomial of degree $k_{j}$. By direct calculation it is easy to see that $\left\|\tilde{h}_{k}(y)\right\|_{L^{2}\left(\mathbb{R}^{n}, d y\right)}=1$.

We now collect some of the results which were obtained in [167] and which we shall use later. In [167] N. Vasilevski introduced the following unitary operators:

$$
\begin{aligned}
& U_{1}: L^{2}\left(\mathbb{C}^{n}, d \mu\right) \longrightarrow L^{2}\left(\mathbb{R}^{n}, d x\right) \otimes L^{2}\left(\mathbb{R}^{n}, d y\right) \\
& \varphi \longrightarrow\left(U_{1} \varphi\right)(x, y):=\pi^{-\frac{n}{2}} e^{-\frac{|x|^{2}+|y|^{2}}{2}} \varphi(x+i y), \\
& \text { - } U_{2}:=I \otimes \mathcal{F}: L^{2}\left(\mathbb{R}^{n}, d x\right) \otimes L^{2}\left(\mathbb{R}^{n}, d y\right) \longrightarrow L^{2}\left(\mathbb{R}^{n}, d x\right) \otimes L^{2}\left(\mathbb{R}^{n}, d y\right) \text {, } \\
& \begin{aligned}
U_{3}=U_{3}^{-1}=U_{3}^{\star}: L^{2}\left(\mathbb{R}^{n}, d x\right) \otimes L^{2}\left(\mathbb{R}^{n}, d y\right) & \longrightarrow \\
\varphi \longrightarrow & L^{2}\left(\mathbb{R}^{n}, d x\right) \otimes L^{2}\left(\mathbb{R}^{n}, d y\right) \\
\varphi & (x, y):= \\
& \varphi\left(\frac{1}{\sqrt{2}}(x+y), \frac{1}{\sqrt{2}}(x-y)\right)
\end{aligned}
\end{aligned}
$$

where $I$ is the identity map and $\mathcal{F}$ is the Fourier transformation on $L^{2}\left(\mathbb{R}^{n}\right)$. The following theorem was established in [167].

Theorem 3.4.1. [167] The unitary operator $U=U_{3} U_{2} U_{1}$ provides an isometric isomorphism of the space $L^{2}\left(\mathbb{C}^{n}, d \mu\right)$ onto $L^{2}\left(\mathbb{R}^{n}, d x\right) \otimes L^{2}\left(\mathbb{R}^{n}, d y\right)$ under which the true- $k$-Fock space is mapped onto $L^{2}\left(\mathbb{R}^{n}, d x\right) \tilde{h}_{k-\mathbf{1}}(y)$ where $\mathbf{1}=(1,1, \cdots, 1)$.

By Generalizing from the case $n=1$ (as proved in [167]) to an arbitrary dimension $n$ and by using the above theorem one can verify that the mapping

$$
R_{(k)}: L^{2}\left(\mathbb{R}^{n}, d x\right) \longrightarrow L^{2}\left(\mathbb{R}^{n}, d x\right) \otimes L^{2}\left(\mathbb{R}^{n}, d y\right)
$$


defined by $R_{(k)} f:=f(x) \tilde{h}_{k-1}(y)$ is an isometric embedding and the adjoint operator

$$
R_{(k)}^{\star}: L^{2}\left(\mathbb{R}^{n}, d x\right) \otimes L^{2}\left(\mathbb{R}^{n}, d y\right) \longrightarrow L^{2}\left(\mathbb{R}^{n}, d x\right)
$$

is given by

$$
\left(R_{(k)}^{\star} \varphi\right)(x)=\int_{\mathbb{R}^{n}} \varphi(x, y) \tilde{h}_{k-\mathbf{1}}(y) d y .
$$

We define the operator $\tilde{R}_{(k)}:=R_{(k)}^{\star} U$ on $L^{2}\left(\mathbb{C}^{n}, d \mu\right)$, and use the same techniques as in [167] for the case $n=1$ to prove the following theorem.

Theorem 3.4.2. The restriction

$$
\tilde{R}_{(k)} \mid F_{(k)}^{2}\left(\mathbb{C}^{n}\right): F_{(k)}^{2}\left(\mathbb{C}^{n}\right) \longrightarrow L^{2}\left(\mathbb{R}^{n}, d x\right)
$$

and the adjoint operator

$$
\tilde{R}_{(k)}^{\star}=U^{\star} R_{(k)}: L^{2}\left(\mathbb{R}^{n}, d x\right) \longrightarrow F_{(k)}^{2}\left(\mathbb{C}^{n}\right)
$$

are isometric isomorphisms. Furthermore, it holds

$$
\tilde{R}_{(k)} \tilde{R}_{(k)}^{\star}=I: L^{2}\left(\mathbb{R}^{n}, d x\right) \longrightarrow L^{2}\left(\mathbb{R}^{n}, d x\right),
$$

and

$$
\tilde{R}_{(k)}^{\star} \tilde{R}_{(k)}=P_{(k)}: L^{2}\left(\mathbb{C}^{n}, d \mu\right) \longrightarrow F_{(k)}^{2}\left(\mathbb{C}^{n}\right)
$$

Let us introduce the notion of true- $k$-Toeplitz operators on the true- $k$-Fock space $F_{(k)}^{2}\left(\mathbb{C}^{n}\right)$.

Definition 3.4.1. Let $h$ be a bounded function on $\mathbb{C}^{n}$ then the true-k-Toeplitz operator $T_{h}^{(k)}$ is defined on $F_{(k)}^{2}\left(\mathbb{C}^{n}\right)$ as

$$
T_{h}^{(k)}:=P_{(k)} M_{h},
$$

where $M_{h}$ is the multiplication operator by $h$.

Note that for $k=\mathbf{1}=(1,1, \cdots, 1)$ we have $F_{(\mathbf{1})}^{2}\left(\mathbb{C}^{n}\right)=\otimes_{j=1}^{n} \mathcal{H}_{1}^{2}(\mathbb{C})=\mathcal{H}_{1}^{2}\left(\mathbb{C}^{n}\right)$ and $T_{h}^{(\mathbf{1})}:=P_{(\mathbf{1})} M_{h}$ is the usual Toeplitz operator on $\mathcal{H}_{1}^{2}\left(\mathbb{C}^{n}\right)$.

Using Theorem 3.4.2 it is easy to see that the true- $k$-Toeplitz operator $T_{h}^{(k)}$ on $F_{(k)}^{2}\left(\mathbb{C}^{n}\right)$ is unitary equivalent to the operator

$$
\begin{aligned}
\tilde{R}_{(k)} T_{h}^{(k)} \tilde{R}_{(k)}^{\star}=\tilde{R}_{(k)} P_{(k)} M_{h} \tilde{R}_{(k)}^{\star} & =\tilde{R}_{(k)} \tilde{R}_{(k)}^{\star} \tilde{R}_{(k)} M_{h} \tilde{R}_{(k)}^{\star} \\
& =\tilde{R}_{(k)} M_{h} \tilde{R}_{(k)}^{\star} \\
& =R_{(k)}^{\star} U M_{h} U^{\star} R_{(k)}:=S_{h} .
\end{aligned}
$$

For a special kind of bounded symbols $\theta$ we prove that the above operator $S_{\theta}$ is a composition of a shift and a multiplication operator on $L^{2}\left(\mathbb{R}^{n}\right)$. 
Theorem 3.4.3. Let $k=\left(k_{1}, k_{2}, \cdots, k_{n}\right)$ be a tuple of positive integers and consider a bounded function $\theta$ on $\mathbb{C}^{n}$ of the following form

$$
\theta(z)=\theta(x, y)=a(A(x)) e^{i u \cdot y}
$$

where $u \in \mathbb{R}^{n}$ and $A$ is an endomorphism of $\mathbb{R}^{n}$. Then the true-k-Toeplitz operator $T_{\theta}^{(k)}$ on $F_{(k)}^{2}\left(\mathbb{C}^{n}\right)$ is unitary equivalent to the operator $S_{\theta}$ on $L^{2}\left(\mathbb{R}^{n}, d x\right)$ which is given by:

$$
\left(S_{\theta} \psi\right)(x)=\psi\left(x-\frac{u}{\sqrt{2}}\right) \int_{\mathbb{R}^{n}} a\left(A\left(\frac{x+y}{\sqrt{2}}\right)\right) \tilde{h}_{k-\mathbf{1}}\left(y+\frac{u}{\sqrt{2}}\right) \tilde{h}_{k-\mathbf{1}}(y) d y,
$$

for all $\psi \in L^{2}\left(\mathbb{R}^{n}, d x\right)$.

Proof. We want to calculate $\left(S_{\theta} \psi\right)(x):=R_{(k)}^{\star}\left(U M_{\theta} U^{\star} R_{(k)} \psi\right)(x)$. First we calculate the operator $U M_{\theta} U^{\star}$. It is easy to check $U_{1} M_{\theta} U_{1}^{\star}=M_{\theta}$. Now let $\phi=U_{2}(f \otimes g)=f \otimes \mathcal{F}(g) \in$ $L^{2}\left(\mathbb{R}^{n}, d x\right) \otimes L^{2}\left(\mathbb{R}^{n}, d y\right)$ then

$$
\begin{aligned}
U_{2} M_{\theta} U_{2}^{\star} \phi & =[I \otimes \mathcal{F}] a(A(x)) e^{i u \cdot y} f \otimes g \\
& =a(A(x)) f \otimes \mathcal{F}\left(g(\cdot) e^{i u(\cdot)}\right)(y) \\
& =a(A(x)) f \otimes \mathcal{F}(g)(y-u)=a(A(x)) \phi(x, y-u) .
\end{aligned}
$$

For convenience we write $(\tau \phi)(x, y):=\phi(x, y-u)$. We shall now apply the operator $U M_{\theta} U^{\star}$ on an arbitrary element $\varphi \in L^{2}\left(\mathbb{R}^{n}, d x\right) \otimes L^{2}\left(\mathbb{R}^{n}, d y\right)$ :

$$
\begin{aligned}
\left(U M_{\theta} U^{\star} \varphi\right)(x, y)=U_{3} a(A(x)) \tau U_{3}^{\star} \varphi & =U_{3} a(A(x)) \tau \varphi\left(\frac{x+y}{\sqrt{2}}, \frac{x-y}{\sqrt{2}}\right) \\
& =U_{3} a(A(x)) \varphi\left(\frac{x+y-u}{\sqrt{2}}, \frac{x-y+u}{\sqrt{2}}\right) \\
& =a\left(A\left(\frac{x+y}{\sqrt{2}}\right)\right) \varphi\left(x-\frac{u}{\sqrt{2}}, y+\frac{u}{\sqrt{2}}\right) .
\end{aligned}
$$

Therefore, for any $\psi \in L^{2}\left(\mathbb{R}^{n}, d x\right)$ we obtain

$$
\begin{aligned}
\left(S_{\theta} \psi\right)(x) & =R_{(k)}^{\star}\left(U M_{\theta} U^{\star} R_{(k)} \psi\right)(x) \\
& =\int_{\mathbb{R}^{n}} U M_{\theta} U^{\star}\left(R_{(k)} \psi\right)(x, y) \tilde{h}_{k-\mathbf{1}}(y) d y \\
& =\int_{\mathbb{R}^{n}} a\left(A\left(\frac{x+y}{\sqrt{2}}\right)\right)\left(R_{(k)} \psi\right)\left(x-\frac{u}{\sqrt{2}}, y+\frac{u}{\sqrt{2}}\right) \tilde{h}_{k-\mathbf{1}}(y) d y \\
& =\psi\left(x-\frac{u}{\sqrt{2}}\right) \int_{\mathbb{R}^{n}} a\left(A\left(\frac{x+y}{\sqrt{2}}\right)\right) \tilde{h}_{k-\mathbf{1}}\left(y+\frac{u}{\sqrt{2}}\right) \tilde{h}_{k-\mathbf{1}}(y) d y .
\end{aligned}
$$

We are able now to construct commutative true- $k$-Toeplitz operators.

Theorem 3.4.4. Consider two bounded functions on $\mathbb{C}^{n}$

$$
\theta(z)=\theta(x, y)=a(A(x)) e^{i u \cdot y} \quad \text { and } \quad \gamma(z)=\gamma(x, y)=b(B(x)) e^{i t \cdot y},
$$

where $A$ and $B$ are endomorphisms of $\mathbb{R}^{n}, t \in \operatorname{ker} A$ and $u \in \operatorname{ker} B$. Then for any tuple $k=$ $\left(k_{1}, k_{2}, \cdots, k_{n}\right)$ of positive integers the two true-k-Toeplitz operators $T_{\theta}^{(k)}$ and $T_{\gamma}^{(k)}$ commute on the true- $k$-Fock space $F_{(k)}^{2}\left(\mathbb{C}^{n}\right)$. 
Proof. By Theorem 3.4.3 we know that $T_{\theta}^{(k)} T_{\gamma}^{(k)}$ is unitary equivalent to the operator $S_{\theta} S_{\gamma}$ on $L^{2}\left(\mathbb{R}^{n}, d x\right)$ which is given by:

$$
\begin{aligned}
\left(S_{\theta} S_{\gamma} \varphi\right)(x)= & S_{\theta}\left(S_{\gamma} \varphi\right)(x)=\left(S_{\gamma} \varphi\right)\left(x-\frac{u}{\sqrt{2}}\right) \int_{\mathbb{R}^{n}} a\left(A\left(\frac{x+y}{\sqrt{2}}\right)\right) \tilde{h}_{k-\mathbf{1}}\left(y+\frac{u}{\sqrt{2}}\right) \tilde{h}_{k-\mathbf{1}}(y) d y \\
= & \varphi\left(x-\frac{u}{\sqrt{2}}-\frac{t}{\sqrt{2}}\right) \int_{\mathbb{R}^{n}} b\left(B\left(\frac{x-\frac{u}{\sqrt{2}}+v}{\sqrt{2}}\right)\right) \tilde{h}_{k-\mathbf{1}}\left(v+\frac{t}{\sqrt{2}}\right) \tilde{h}_{k-\mathbf{1}}(v) d v \\
& \times \int_{\mathbb{R}^{n}} a\left(A\left(\frac{x+y}{\sqrt{2}}\right)\right) \tilde{h}_{k-\mathbf{1}}\left(y+\frac{u}{\sqrt{2}}\right) \tilde{h}_{k-\mathbf{1}}(y) d y .
\end{aligned}
$$

On the other hand $T_{\gamma}^{(k)} T_{\theta}^{(k)}$ is unitary equivalent to the operator $S_{\gamma} S_{\theta}$ on $L^{2}\left(\mathbb{R}^{n}, d x\right)$ :

$$
\begin{aligned}
\left(S_{\gamma} S_{\theta} \varphi\right)(x)= & S_{\gamma}\left(S_{\theta} \varphi\right)(x)=\left(S_{\theta} \varphi\right)\left(x-\frac{t}{\sqrt{2}}\right) \int_{\mathbb{R}^{n}} b\left(B\left(\frac{x+v}{\sqrt{2}}\right)\right) \tilde{h}_{k-\mathbf{1}}\left(v+\frac{t}{\sqrt{2}}\right) \tilde{h}_{k-\mathbf{1}}(v) d v \\
= & \varphi\left(x-\frac{t}{\sqrt{2}}-\frac{u}{\sqrt{2}}\right) \int_{\mathbb{R}^{n}} a\left(A\left(\frac{x-\frac{t}{\sqrt{2}}+y}{\sqrt{2}}\right)\right) \tilde{h}_{k-\mathbf{1}}\left(y+\frac{u}{\sqrt{2}}\right) \tilde{h}_{k-\mathbf{1}}(y) d y \\
& \times \int_{\mathbb{R}^{n}} b\left(B\left(\frac{x+v}{\sqrt{2}}\right)\right) \tilde{h}_{k-\mathbf{1}}\left(v+\frac{t}{\sqrt{2}}\right) \tilde{h}_{k-\mathbf{1}}(v) d v .
\end{aligned}
$$

It is easy now to see that the two equations (3.4.1) and (3.4.2) are equal whenever $t \in \operatorname{ker} A$ and $u \in \operatorname{ker} B$.

Remark 3.4.1. Let $\theta$ and $\gamma$ be the two functions defined in the above theorem. Then the Toeplitz operators $\mathcal{T}_{\theta}^{s}$ and $\mathcal{T}_{\gamma}^{s}$ commute on each Segal-Bargmann space $\mathcal{H}_{s}^{2}\left(\mathbb{C}^{n}\right)$. Indeed, the above theorem shows that the Toeplitz operators $T_{\theta}$ and $T_{\gamma}$ commute on $\mathcal{H}_{1}^{2}\left(\mathbb{C}^{n}\right)$ as well as the operators $T_{\theta\left(\frac{\dot{\sqrt{s}}}{}\right)}$ and $T_{\gamma\left(\frac{\dot{\sqrt{s}}}{)}\right.}$ for any $s>0$. Now by a slight generalization of Eq. (2.4.20) for the case of an arbitrary dimension $n$ one can easily verify that for any measurable function $h$ we have

$$
\mathcal{T}_{h}^{s} g(z)=\left[T_{h\left(\frac{\dot{\sqrt{s}}}{s}\right.} g\left(\frac{\cdot}{\sqrt{s}}\right)\right](\sqrt{s} z), \quad \text { for all } \quad g \quad \text { s.t. } \quad h g \in L^{2}\left(\mathbb{C}, d \mu_{(s)}\right) .
$$

Applying the above equation to the product operator $\mathcal{T}_{\theta}^{s} \mathcal{T}_{\gamma}^{s}$ one obtains

$$
\begin{aligned}
& \mathcal{T}_{\theta}^{s} \mathcal{T}_{\gamma}^{s} g(z)=\left[T_{\theta\left(\frac{\dot{\sqrt{s}}}{)}\right.}\left[\left[T_{\gamma\left(\frac{\dot{\sqrt{s}}}{)}\right.} g\left(\frac{\cdot}{\sqrt{s}}\right)\right](\cdot)\right]\right](\sqrt{s} z) \\
& =\left[T_{\gamma\left(\frac{\dot{\sqrt{s}}}{)}\right.}\left[\left[T_{\theta\left(\frac{\dot{\sqrt{s}}}{)}\right)} g\left(\frac{\cdot}{\sqrt{s}}\right)\right](\cdot)\right]\right](\sqrt{s} z) \\
& =\mathcal{T}_{\gamma}^{s} \mathcal{T}_{\theta}^{s} g(z) \quad \text { for all } \quad g \in \mathcal{H}_{s}^{2}\left(\mathbb{C}^{n}\right)
\end{aligned}
$$

Theorem 3.4.4 (respectively Remark 3.4.1) allows us to generate commutative $C^{\star}$-algebras of true- $k$-Toeplitz operators (respectively of Toeplitz operators).

Corollary 3.4.1. Let $\mathcal{H}$ be a linear subspace of $\mathbb{R}^{n}$ then for each tuple $k=\left(k_{1}, k_{2}, \cdots, k_{n}\right)$ of positive integers the $C^{\star}$-algebra generated by the set

$$
\left\{T_{a(A(x)) e^{i u \cdot y}}^{(k)} \mid a \in L^{\infty}\left(\mathbb{R}^{n}\right), A \text { is an endomorphism of } \mathbb{R}^{n} \text { with } \operatorname{ker} A=\mathcal{H} \text { and } u \in \mathcal{H}\right\}
$$

is commutative. 
Corollary 3.4.2. Let $\mathcal{H}$ be a linear subspace of $\mathbb{R}^{n}$ then the $C^{\star}$-algebra generated by the set $\left\{\mathcal{T}_{a(A(x)) e^{i u \cdot y}}^{s} \mid a \in L^{\infty}\left(\mathbb{R}^{n}\right), A\right.$ is an endomorphism $\mathbb{R}^{n}$ with ker $A=\mathcal{H}$ and $\left.u \in \mathcal{H}\right\}$ is commutative.

Now we combine the above result of commutative $C^{\star}$-algebra with the result in Section 3.3 to form a more general types of commutative Banach algebras genrated by Toeplitz operators.

Let $s>0$ be arbitrary and consider the Segal-Bargmann space $\mathcal{H}_{s}^{2}\left(\mathbb{C}^{n}\right)$. Fix a number $l \in\{0,1, \cdots, n\}$ and a tuple $k=\left(k_{1}, \cdots, k_{m}\right) \in \mathbb{N}^{m}$ such that $|k|=l$. Consider a tuple $h=\left(h_{1}, \cdots, h_{m}\right)$ with $h_{j}=0$ if $k_{j}=1$ and $1 \leq h_{j} \leq k_{j}-1$ and when $k_{j_{0}}=k_{j_{1}}$ for certain $j_{0}<j_{1}$ put $h_{j_{0}} \leq h_{j_{1}}$. Moreover, for each $j=1, \cdots, m$ let $p_{(j)}, q_{(j)} \in \mathbb{N}_{0}^{k_{j}}$ to be of the following form

$$
p_{(j)}=\left(p_{j, 1}, \cdots, p_{j, h_{j}}, 0, \cdots, 0\right) \quad q_{(j)}=\left(0, \cdots, 0, q_{j, h_{j}+1}, \cdots, q_{j, k_{j}}\right), \text { with }\left|p_{(j)}\right|=\left|q_{(j)}\right| .
$$

Note that the numbers $p_{j, 1}, \cdots, p_{j, h_{j}}, q_{j, h_{j}+1}, \cdots, q_{j, k_{j}}$ are arbitrary in $\mathbb{N}_{0}$. Now with those $p_{(j)}, q_{(j)}$ as defined above put

$$
p=\left(p_{(1)}, \cdots, p_{(m)}\right), \quad q=\left(q_{(1)}, \cdots, q_{(m)}\right) \in \mathbb{N}^{l} .
$$

Let us decompose the complex space $\mathbb{C}^{n}$ as the product $\mathbb{C}^{n}=\mathbb{C}^{l} \times \mathbb{C}^{n-l}$ and write $z=\left(z^{\prime}, z^{\prime \prime}\right) \in$ $\mathbb{C}^{l} \times \mathbb{C}^{n-l}$. Moreover, we represent $z^{\prime} \in \mathbb{C}^{l}$ in the polar coordinates $r_{j} \xi_{(j)}$ with $r_{j}=\left|z_{(j)}^{\prime}\right|, \xi_{(j)} \in$ $\mathbb{S}^{2 k_{j}-1}$ and we write $\xi=\left(\xi_{(1)}, \cdots, \xi_{(m)}\right)$. We denote by $\mathcal{R}_{k}^{l}(h)$ the space of bounded $k$-quasihomogeneous symbols

$$
\varphi\left(r_{1}, \cdots, r_{m}\right) \xi^{p} \bar{\xi}^{q}
$$

where $p, q$ are given by the above form. Furthermore, for $z^{\prime \prime} \in \mathbb{C}^{n-l}$ we decompose $z^{\prime \prime}$ in its real and imaginary part $z^{\prime \prime}=x+i y$.

With the notation used above, we are now able to construct new commutative Banach algebras generated by Toeplitz operators.

Corollary 3.4.3. Let $l \in\{0,1, \cdots, n\}$ be fixed. Then for each linear subspace $\mathcal{H} \subseteq \mathbb{R}^{n-l}$ and each tuple $k=\left(k_{1}, \cdots, k_{m}\right) \in \mathbb{N}^{m}$ such that $|k|=l$ and each tuple $h$ as described above the Banach algebra generated by the set

$$
\begin{aligned}
& \left\{\mathcal{T}_{\varphi \xi^{p} \bar{\xi}}^{s} a(A(x)) e^{i u \cdot y} \mid \varphi \xi^{p} \bar{\xi}^{q} \in \mathcal{R}_{k}^{l}(h), a \in L^{\infty}\left(\mathbb{R}^{n-l}\right), u \in \mathcal{H}, A \in \mathcal{L}\left(\mathbb{R}^{n-l}\right) \text { s.t. } \operatorname{ker} A=\mathcal{H}\right\} \\
& \text { is commutative. }
\end{aligned}
$$

Proof. Let $g=g_{1} \otimes g_{2} \in \mathcal{H}_{s}^{2}\left(\mathbb{C}^{n}\right)=\mathcal{H}_{s}^{2}\left(\mathbb{C}^{l}\right) \otimes \mathcal{H}_{s}^{2}\left(\mathbb{C}^{n-l}\right)$. Then for any Toeplitz operator of the above form one can easily check that

$$
\left[\mathcal{T}_{\varphi \xi^{p} \bar{\xi}^{q} a(A(x)) e^{i u \cdot y}}^{s} g\right](z)=\left[\mathcal{T}_{\varphi \xi^{p} \bar{\xi}^{q}}^{s} g_{1}\right]\left(z^{\prime}\right) \cdot\left[\mathcal{T}_{a(A(x)) e^{i u y}}^{s} g_{2}\right]\left(z^{\prime}\right)
$$


where $\mathcal{T}_{\varphi \xi^{p} \bar{\xi}^{q}}^{s}$ and $\mathcal{T}_{a(A(x)) e^{i u y}}^{s}$ are the Toeplitz operators on $\mathcal{H}_{s}^{2}\left(\mathbb{C}^{l}\right)$ and $\mathcal{H}_{s}^{2}\left(\mathbb{C}^{n-l}\right)$, respectively. The corollary follows by an application of Corollary 3.3.3 to the case of the Segal-Bargmann space $\mathcal{H}_{s}^{2}\left(\mathbb{C}^{l}\right)$ and another application of Corollary 3.4.2 to the case of the Segal-Bargmann space $\mathcal{H}_{s}^{2}\left(\mathbb{C}^{n-l}\right)$.

Note that, in the above corollary, when $l=n$ (respectively $l=0$ ) this is the commutative Banach-algebra (respectively $C^{\star}$-algebra) obtained in Corollary 3.3.3 (respectively in Corollary 3.4.2).

Finally, we would like to mention some open problems which are inspired by our results.

1. With the notation used in Section 3.2, it is clear that for any tuple $k=\left(k_{1}, \cdots, k_{m}\right) \in \mathbb{N}^{m}$ with $|k|=n$ the set

$$
\mathcal{A}_{k}^{s}\left(\mathbb{C}^{n}\right):=\left\{\tilde{\mathcal{T}}_{\varphi}^{s}, \varphi \in L_{k}^{s} \text { such that } \tilde{\mathcal{T}}_{\varphi}^{s} \in \mathcal{L}\left(\mathcal{H}_{s}^{2}\right)\right\}
$$

is contained in the commutative $C^{\star}$-algebra

$$
\begin{aligned}
\mathcal{A}_{k}=\left\{T \in \mathcal{L}\left(\mathcal{H}_{s}^{2}\right) \mid T z^{\alpha}=\delta_{\alpha} z^{\alpha} \text { where } \delta_{\alpha}=\delta_{\beta}\right. \\
\text { whenever } \left.\left|\alpha_{(j)}\right|=\left|\beta_{(j)}\right| \text { for all } j=1, \cdots, m \text { and all } \alpha, \beta \in \mathbb{N}_{0}^{n}\right\} .
\end{aligned}
$$

Analogous to the case $k=(n)$ as considered in Corollary 3.2.1, one may ask wether it is still true that $C_{k}^{s}\left(\mathbb{C}^{n}\right) \equiv C_{k}$ thus being also a commutative $C^{\star}$-algebra. In fact, it is easy to see that such a problem reduces to the following higher dimensional moment problem:

Let $\left\{\delta_{\alpha}\right\}_{\alpha \in \mathbb{N}_{0}^{n}}$ be such that $\delta_{\alpha}=\delta_{\left(\left|\alpha_{(1)}\right|, \cdots,\left|\alpha_{(m)}\right|\right)}$. Find a function $g\left(r_{1}, \cdots, r_{m}\right)$ such that

$$
\delta_{\alpha}=\int_{\mathbb{R}_{+}^{m}} g\left(r_{1}, \cdots, r_{m}\right) \prod_{j=1}^{m} r_{j}^{\left|\alpha_{(j)}\right|} d r_{j} \quad \text { for all } \alpha \in \mathbb{N}_{0}^{n}
$$

and that

$$
\frac{g\left(r_{1}^{2}, \cdots, r_{m}^{2}\right) e^{s r^{2}}}{\prod_{j=1}^{m} r_{j}^{2 k_{j}-\left|\beta_{(j)}\right|}} \in L^{1}\left(\mathbb{R}_{+}^{m}, d r\right) \quad \text { for all } \beta=\left(\beta_{(1)}, \cdots, \beta_{(m)}\right) \in \mathbb{N}_{0}^{n} .
$$

2. As mentioned in Section 2.1, in [66] Z. Cučcković and N. Rao proved that if $\varphi_{1}, \varphi_{2} \in$ $L^{\infty}(\mathbb{D})$ such that $\varphi_{1}$ is non-constant and radial then the Toeplitz operators $T_{\varphi_{1}}$ and $T_{\varphi_{2}}$ commute on $A^{2}(\mathbb{D})$ if and only if $\varphi_{2}$ is radial. Later on, in [181] Ze-Hua Zhou and XingTang Dong showed that if $p, q \in \mathbb{N}_{0}^{n}$ are orthogonal multi-indices and $\varphi_{1}, \varphi_{2} \in L^{\infty}\left(\mathbb{B}^{n}\right)$ are radial such that $\varphi_{1}$ is non-constant and $\varphi_{2} \neq 0$ then $T_{\varphi_{1}}$ and $T_{\varphi_{2} \xi^{p} \bar{\xi}^{q}}$ commute on the un-weighted Bergman space $A^{2}\left(\mathbb{B}^{n}\right)$ if and only if $|p|=|q|$. Finally, in [172] N. Vasilevski generalized the previously result to the case of $k$-quasi-radial functions and standard weighted Bergman spaces $A_{\lambda}^{2}\left(\mathbb{B}^{n}\right)$ over the unit ball. Roughly speaking, given a 
tuple $k=\left(k_{1}, \cdots, k_{m}\right) \in \mathbb{N}^{m}$ with $|k|=n$, a pair of orthogonal multi-indices $p, q \in \mathbb{N}_{0}^{n}$, and two $k$-quasi-radial functions $\varphi_{1}, \varphi_{2} \in L^{\infty}\left(\mathbb{B}^{n}\right)$ such that $\varphi_{1}$ is non-constant and $\varphi_{2} \neq 0$, then $T_{\varphi_{1}}$ and $T_{\varphi_{2} \xi^{p} \bar{\xi}^{q}}$ commute on each weighted Bergman space $A_{\lambda}^{2}\left(\mathbb{B}^{n}\right)$ if and only if $\left|p_{(j)}\right|=\left|q_{(j)}\right|$ for each $j=1, \cdots, m$.

As for such a problems in the case of the Segal-Bargmann space the growth of the symbol near infinity plays an essential role. As explained in Section 1.3, W. Bauer and T. Le proved that if $\varphi_{1}, \varphi_{2} \in \operatorname{Sym}_{>0}\left(\mathbb{C}^{n}\right):=\cap_{s>0} \mathcal{E}_{s}$ such that $\varphi_{1}$ is non-trivial and radial then $\mathcal{T}_{\varphi_{1}}^{s}$ and $\mathcal{T}_{\varphi_{2}}^{s}$ commute if and only if $\varphi_{2}\left(e^{i \theta} z\right)=\varphi_{2}(z)$ for a.e. $\theta \in \mathbb{R}$ and a.e. $z \in \mathbb{C}^{n}$ [16]. In particular, if $\varphi_{2}(z)=\varphi_{3}(r) \xi^{p} \bar{\xi}^{q}$ for some orthogonal multi-indices $p, q \in \mathbb{N}_{0}^{n}$ and some radial function $\varphi_{3}$ then $\mathcal{T}_{\varphi_{1}}^{s}$ and $\mathcal{T}_{\varphi_{2}}^{s}$ commute if and only if $|p|=|q|$. Analogous to this case, one may ask if the following is till true:

Let $k=\left(k_{1}, \cdots, k_{m}\right) \in \mathbb{N}^{m}$ with $|k|=n$ and fix a pair of orthogonal multi-indices $p, q \in \mathbb{N}_{0}^{n}$. Consider two $k$-quasi-radial functions $\varphi_{1}, \varphi_{2} \in \operatorname{Sym}_{>0}\left(\mathbb{C}^{n}\right)$ such that $\varphi_{1}$ is non-constant and $\varphi_{2} \neq 0$ then $\mathcal{T}_{\varphi_{1}}^{s}$ and $\mathcal{T}_{\varphi_{2} \xi^{p} \bar{\xi}^{q}}^{s}$ commute on each Segal-Bargmann space $\mathcal{H}_{s}^{2}\left(\mathbb{C}^{n}\right)$ if and only if $\left|p_{(j)}\right|=\left|q_{(j)}\right|$ for each $j=1, \cdots, m$. The sufficient condition is proved by Proposition 3.3.1, however the necessary condition is still open for further research. 


\section{Chapter 4}

\section{„Heat kernel” for Toeplitz operators}

We employ Berezin's result in [29] to calculate the heat kernel of a certain class of elliptic and sub-elliptic partial differential operators. Via the Bargmann transform which maps $L^{2}\left(\mathbb{R}^{n}\right)$ isometrically onto $H^{2}\left(\mathbb{C}^{n}\right):=\mathcal{H}_{1}^{2}\left(\mathbb{C}^{n}\right)$ every partial differential operator $\mathcal{L}$ on $L^{2}\left(\mathbb{R}^{n}\right)$ with polynomial coefficients is unitary equivalent to a Toeplitz operator $T_{f}$ on $H^{2}\left(\mathbb{C}^{n}\right)$ where $f$ is a polynomial over $\mathbb{C}^{n}$ in the complex variables $z$ and $\bar{z}$. We show that when $f(z, \bar{z})=z A \bar{z}$ and $A$ is a $n \times n$ positive semidefinite matrix then the one parameter semi-group $e^{-t T_{f}}$ can be calculated explicitly by Berezin's formula (4.1.1). In this case, it turns out that the operator $e^{-t T_{f}}$ is also a Toeplitz operator on $H^{2}\left(\mathbb{C}^{n}\right)$ and the ,heat kernel" of $T_{f}$ defined on $\mathbb{R}_{+} \times \mathbb{C}^{2 n}$ is simply obtained (c.f. Theorem 4.3.5). Finally, the heat kernel of the operator $\mathcal{L}$ defined on $\mathbb{R}_{+} \times \mathbb{R}^{2 n}$ is deduced via an application of the inverse Bargmann transform. We illustrate our method by obtaining the heat kernel of the Hermite operator on $\mathbb{R}^{n}$ as well as that of the isotropic twisted Laplacian on $\mathbb{R}^{n}$ (here $n=2 N$ with $N \in \mathbb{N}$ is arbitrary). Moreover, using this approach together with the partial Fourier transformation we calculate the heat kernel of the Grusin operator on $\mathbb{R}^{n+1}$ as well as that of the sub-Laplace operator on the $(2 N+1)$-dimensional Heisenberg group $\mathbb{H}_{(2 N+1)}$.

\subsection{Introduction}

Several attempts for expressing the heat kernel for the sub-Laplace operator $\Delta_{\text {sub }}$ on nilpotent Lie groups appeared in many papers $[24-27,58,59,67,85,86,110,116,148]$. In [88], B. Gaveau constructed the heat kernel for $\Delta_{\text {sub }}$ on free 2-step nilpotent Lie groups using the complex Hamilton-Jacobi method (see also [25-27, 55]). By the left invariance of $\Delta_{\text {sub }}$ on a nilpotent Lie group $(M, \star)$ the heat kernel $k(t, x, y)$ can be described by a smooth function $k_{t}(x)$ on $\mathbb{R}_{+} \times M$ in the form $k(t, x, y)=k_{t}\left(y^{-1} \star x\right)$. The complex Hamilton-Jacobi method assumes that the kernel $k_{t}(x)$ has a certain integral form which reflects the physical phenomena. More precisely, the value of $k_{t}(x)$ at a point $x$ and time $t$ is equal to the integral of the heat flowing over a certain class of geodesics starting from the identity element of the group and arriving at $x$ at the time $t$. By solving a certain Hamiltonian system (c.f. Chapter 10 in [56]) the class 
of the geodesics is determined. Another method for calculating the heat kernel for $\Delta_{\text {sub }}$ on free 2-step nilpotent Lie groups uses the symbolic calculus of pseudo-differential operators (c.f. $[115,116])$. This method is also applicable to strongly elliptic operators and some sub-elliptic operators (c.f. Chapter 15 in [56]). The heat kernel of $\Delta_{\text {sub }}$ is usually given in an integral form (c.f. Theorem 15.4.8 in [56]). In the case of connected and simply connected free 2step nilpotent Lie groups the integral runs over $\mathbb{R}^{n+\frac{n(n-1)}{2}}$ where $n$ is the dimension of the Lie algebra. Several other methods for calculating the kernel of $\Delta_{\text {sub }}$ are explained in [56].

The easiest example of a 2-step nilpotent Lie group is the $(2 N+1)$-dimensional Heisenberg group $\mathbb{H}_{(2 N+1)}$. In [110], A. Hulanicki was the first to give an explicit integral formula for the heat kernel of the sub-Laplacian on $\mathbb{H}_{(2 N+1)}$ using a probabilistic argument. The argument involves some harmonic analysis techniques especially Mehler's formula for the Hermite functions (c.f. [84, 164]). In this chapter, we investigate the heat kernel for a class of elliptic and sub-elliptic differential operators using Toeplitz operator theory techniques. Our method can be used for finding the heat kernel of the sub-Laplacian on $\mathbb{H}_{(2 N+1)}$ but it is also applicable for other differential operators (c.f. Examples 4.3.1, 4.3.2 and 4.3.3, Corollary 4.3.2 and Theorem 4.3.7) with polynomial coefficients. However, it is still not clear how to characterize completely the class of partial differential operators for which their heat kernel can be obtained by this method. In fact, our approach is somewhat limited since it is heavily based on the symbol of the Toeplitz operator associated to the differential operator via the Bargmann transform. The method we use relies on the partial Fourier transform, Bargmann transform and on Berezin's method for the construction of the exponential of an essentially selfadjoint Toeplitz operator having a positive symbol [29]. Let us explain these tools in more details.

Consider a partial differential operator $\mathcal{L}$ on $L^{2}\left(\mathbb{R}^{n+1}\right)$ whose function coefficients are independent of the last variable. The partial Fourier transform with respect to the last variable is used to eliminate this variable in the operator $\mathcal{L}$. This transform in some of the examples is used to reduce a sub-elliptic operator to an elliptic one (c.f. Chapter 5 in [56]). The problem is then reduced to find the heat kernel of this elliptic operator having fewer variables. Under certain conditions (c.f. Proposition 4.3.1) the heat kernel of the sub-elliptic operator can be obtained by an application of the inverse partial Fourier transform to the heat kernel of the elliptic operator.

Suppose that the operator obtained by applying the partial Fourier transform on $\mathcal{L}$ is a partial differential operator with polynomial coefficients and let us denote it by $\mathcal{L}_{1}$. Via the Bargmann transform $\mathcal{L}_{1}$ is unitary equivalent to a Toeplitz operator $T_{f}$ on $H^{2}\left(\mathbb{C}^{n}\right)$ where $f$ is a polynomial over $\mathbb{C}^{n}$. The problem of finding the heat kernel of $\mathcal{L}_{1}$ on $L^{2}\left(\mathbb{R}^{n}\right)$ is then transformed to a similar problem of finding the ,heat kernel” of $T_{f}$ on $H^{2}\left(\mathbb{C}^{n}\right)$ (c.f. Definition 4.3.3 for the notion of the ,"heat kernel” of Toeplitz operators). In fact, the heat kernel of $\mathcal{L}_{1}$ on $L^{2}\left(\mathbb{R}^{n}\right)$ can be easily related to the heat kernel of $T_{f}$ via an application of the inverse Bargmann transform.

There is no general method to calculate the ,heat kernel” of the Toeplitz operator $T_{f}$ explicitly. However, in the case where $f$ is positive and $T_{f}$ is selfadjoint the operator $e^{-s T_{f}}$ exists for all $s>0$ and is given by Berezin's formula (c.f. [29])

$$
e^{-s T_{f}}=\lim _{N \longrightarrow \infty}\left(T_{e^{-\frac{s}{N} f}}\right)^{N}
$$


where the above limit is understood in the strong sense. In this case the „heat kernel” of the operator $T_{f}$ on $H^{2}\left(\mathbb{C}^{n}\right)$ is obtained by a simple expression involving the exponential operator $e^{-s T_{f}}$ and the reproducing kernel of the Segal-Bargmann space (c.f. Theorem 4.3.5). Therefore, the main point is to compute the higher product of Toeplitz operators $\left(T_{e^{-\frac{s}{N}} f}\right)^{N}$ where $s>0$ and $N \in \mathbb{N}$. This product is in general expressed as a multiple integral over $\mathbb{C}^{n N}$ (c.f. [29]) and in some cases it can be reduced to a simple integral over $\mathbb{C}^{n}$. In particular, if we consider $f(z)=z A \bar{z}$, where $z, \bar{z} \in \mathbb{C}^{n}$ and $I+\frac{s}{N} A$ are positive definite Hermitian matrices for all $s>0$ and $N \in \mathbb{N}$, then each product $\left(T_{e^{-\frac{s}{N} f}}\right)^{N}$ is simply a Toeplitz operator whose symbol involves $s$ and $N$. Moreover, using Eq. (4.1.1) the exponential operator $e^{-s T_{f}}$ is also a Toeplitz operator with an explicitly given symbol involving the time parameter $s$. In this case, the ,heat kernel" of the Toeplitz operator $T_{f}$ is easily computed and via the inverse Bargmann transform and the inverse partial Fourier transform the heat kernel of the partial differential operator $\mathcal{L}$ on $L^{2}\left(\mathbb{R}^{n+1}\right)$ is obtained.

In this chapter, we aim to give a proof of the above approach for calculating the heat kernel of a class of elliptic and sub-elliptic partial differential operators. We illustrate the method by calculating the heat kernel for the Hermite operator on $\mathbb{R}^{n}$ and for the isotropic twisted Laplacian on $\mathbb{R}^{n}$ (here $n=2 N$ with $N \in \mathbb{N}$ is arbitrary). We also give an explicit integral expression for the heat kernel of the Grusin operator on $\mathbb{R}^{n+1}$ and the sub-Laplace operator on $\mathbb{H}_{(2 N+1)}$. We note that the exact form of these kernels have been known since some time but our method works for other cases too.

Chapter 4 is organized as follows: In Section 4.2, we start by introducing a family of Bargmann transforms $\left\{\beta_{t}\right\}_{t>0}$ for which each $\beta_{t}$ maps $L^{2}\left(\mathbb{R}^{n}\right)$ isometrically onto $H^{2}\left(\mathbb{C}^{n}\right)$. The parameter $t>0$ is essential and will be used later to replace the variable which was eliminated through the application of the partial Fourier transform to the operator $\mathcal{L}$. Via the Bargmann transform $\beta_{t}$ we represent each suitable integral operator acting on the Schwartz space $\mathcal{S}\left(\mathbb{R}^{n}\right) \subset L^{2}\left(\mathbb{R}^{n}\right)$ by an integral operator on $H^{2}\left(\mathbb{C}^{n}\right)$ and express its kernel in terms of the Bargmann transform applied to the kernel of the initial operator. We then give an explicit formula for the ,,inverse Bargmann transform" of $e^{z A w}$ where $(z, w) \in \mathbb{C}^{2 n}$ and $A$ is a Hermitian matrix satisfying some conditions (c.f. Proposition 4.2.4). This calculation will be used later to obtain the heat kernel of the sub-Laplacian from the „,heat kernel” of the associated Toeplitz operator obtained via the inverse Bargmann transform. We end the section by introducing the $(2 N+1)$-dimensional Heisenberg group and the sub-Laplacian $\Delta_{\text {sub }}$ acting on it. In Section 4.3, we use the partial Fourier transform to reduce the problem of finding the heat kernel of a partial differential operator to a simpler problem involving fewer variables. Berezin's result is then stated and is applied to Toeplitz operators with symbols of the above mentioned type. The ,heat kernels" of these Toeplitz operators are then calculated and the heat kernel of the Hermite and the isotropic twisted Laplace operators are deduced. Finally, with the help of the partial Fourier transformation we apply these techniques to the Grusin operator and to $\Delta_{\text {sub }}$ and provide the explicit expression of their heat kernels. 


\subsection{The Bargmann transform}

In this section, we introduce a family of Bargmann transform $\left\{\beta_{t}\right\}_{t>0}$ parametrized by the parameter $t>0$. Using Bargmann's result [12], we show that each $\beta_{t}$ maps $L^{2}\left(\mathbb{R}^{n}\right)$ isometrically onto $H^{2}\left(\mathbb{C}^{n}\right)$. For an integral operator $D$ acting on a dense domain of $H^{2}\left(\mathbb{C}^{n}\right)$ we find its corresponding (via the push-forward under $\beta_{t}^{-1} D \beta_{t}$ ) integral operator on $L^{2}\left(\mathbb{R}^{n}\right)$. We shall also calculate the ,,inverse Bargmann transform” of $e^{z A w}$ whenever $A$ is a Hermitian matrix satisfying some conditions. This result will be used in Section 4.3 to calculate the heat kernel of a certain type of sub-elliptic operators. Finally, we introduce the sub-Laplacian on the $(2 N+1)$-dimensional Heisenberg group.

Throughout this chapter and to simplify the notation used here we simply write $\langle\cdot, \cdot\rangle$ (respectively $d \mu$ ) for the inner product $\langle\cdot, \cdot\rangle_{(1)}$ (respectively for the Gaussian measure $d \mu_{(1)}$ ) on the Segal-Bargmann space $H^{2}\left(\mathbb{C}^{n}\right):=\mathcal{H}_{1}^{2}\left(\mathbb{C}^{n}\right)$. For a matrix $A=\left(a_{j k}\right) \in M_{n}(\mathbb{C})$ with complex entries we denote by $\bar{A}:=\left(\overline{a_{j k}}\right), A^{T}:=\left(a_{k j}\right) \in M_{n}(\mathbb{C})$ the complex conjugate and the transpose of $A$, respectively. Moreover, we write $A^{\star}:=\bar{A}^{T}=\left(\overline{a_{k j}}\right)$ for the conjugate transpose of $A$. For $z, w \in \mathbb{C}^{n}$ we will sometimes denote by $z \cdot w$ the usual product $z w=\sum z_{j} w_{j}$. For each $z \in \mathbb{C}^{n}$ we write $K_{z}(u):=e^{u \bar{z}}$ for the reproducing kernel $K^{(1)}(u, z)$ at the point $z$ and for a fixed $t>0$ we put

$$
\Phi_{z}^{t}(x):=t^{\frac{n}{2}}(2 \pi)^{-\frac{n}{4}} \exp \left\{t x \bar{z}-t^{2} \frac{x^{2}}{4}-\frac{\bar{z}^{2}}{2}\right\}
$$

For each $t>0$ one can check that the linear span of $\left\{\Phi_{z}^{t}\right\}_{z \in \mathbb{C}^{n}}$ is norm dense in $L^{2}\left(\mathbb{R}^{n}\right)$ (c.f. Chapter 1 in [84]) and that for any $z, u \in \mathbb{C}^{n}$ the following equality holds:

$$
\left\langle\Phi_{z}^{t}, \Phi_{u}^{t}\right\rangle_{L^{2}\left(\mathbb{R}^{n}\right)}=\left\langle K_{z}, K_{u}\right\rangle=e^{u \bar{z}}
$$

Let us now introduce the Bargmann transform on $L^{2}\left(\mathbb{R}^{n}\right)$.

Definition 4.2.1. For each fixed $t>0$, the Bargmann transform of $f \in L^{2}\left(\mathbb{R}^{n}\right)$ denoted by $\beta_{t} f$ is the entire function defined on $\mathbb{C}^{n}$ by:

$$
\beta_{t} f(z):=\left\langle f, \Phi_{z}^{t}\right\rangle_{L^{2}\left(\mathbb{R}^{n}\right)}=\int_{\mathbb{R}^{n}} f(x) \Phi_{\bar{z}}^{t}(x) d x .
$$

The Bargmann transform $\beta_{\sqrt{2}}$ was first introduced by S. Bargmann in his paper [12] on the structure of the Segal-Bargmann space. Bargmann proved that $\beta_{\sqrt{2}}$ maps $L^{2}\left(\mathbb{R}^{n}\right)$ isometrically onto $H^{2}\left(\mathbb{C}^{n}\right)$ (c.f. also [84]). However, for any $s, t>0$ on can easily check that

$$
\beta_{s}=\beta_{t} \circ U_{\frac{t}{s}}
$$

where $U_{\frac{t}{s}}$ is the unitary operator defined on $L^{2}\left(\mathbb{R}^{n}\right)$ by $\left[U_{\frac{t}{s}} f\right](x):=\left(\frac{t}{s}\right)^{\frac{n}{2}} f\left(\frac{t}{s} x\right)$. This shows that for any $t>0$ the Bargmann transform $\beta_{t}$ maps $L^{2}\left(\mathbb{R}^{n}\right)$ isometrically onto $H^{2}\left(\mathbb{C}^{n}\right)$. Using Equation (4.2.2) together with Equation (2.11) in [12] for the inverse Bargmann transform $\beta_{\sqrt{2}}^{-1}$ one can easily calculate $\beta_{t}^{-1}$ for all $t>0$. In the next theorem, we summarize the above facts and give the explicit expression for the inverse Bargmann transform $\beta_{t}^{-1}$. 
Theorem 4.2.1. For each $t>0$ the Bargmann transform $\beta_{t}$ is an isometry from $L^{2}\left(\mathbb{R}^{n}\right)$ onto $H^{2}\left(\mathbb{C}^{n}\right)$ and the inverse Bargmann transform is given by:

$$
\left[\beta_{t}^{-1} F\right](x)=\int_{\mathbb{C}^{n}} F(z) \Phi_{z}^{t}(x) d \mu(z) \quad \text { for all } F \in H^{2}\left(\mathbb{C}^{n}\right)
$$

Denote by $\mathcal{S}\left(\mathbb{R}^{n}\right) \subset L^{2}\left(\mathbb{R}^{n}\right)$ the Schwartz space over $\mathbb{R}^{n}$. In the following we characterize the entire functions over $\mathbb{C}^{n}$ which are in the range of the Bargmann transform $\beta_{t}\left[\mathcal{S}\left(\mathbb{R}^{n}\right)\right]$.

Proposition 4.2.1. Let $f(z)=\sum_{\alpha \in \mathbb{N}_{0}^{n}} c_{\alpha} z^{\alpha}$ be an entire function over $\mathbb{C}^{n}$. Then for every $t>0$ we have

$$
f \in \beta_{t}\left[\mathcal{S}\left(\mathbb{R}^{n}\right)\right] \Longleftrightarrow \text { for every } M>0, \sup _{\alpha \in \mathbb{N}_{0}^{n}}\left(\left|c_{\alpha}\right| \prod_{j=1}^{n} \alpha_{j}^{M} \sqrt{\alpha_{j} !}\right)<\infty .
$$

Moreover, the space $\beta_{t}\left[\mathcal{S}\left(\mathbb{R}^{n}\right)\right]$ is invariant under the change of variable $z \longmapsto P z$ for any unitary matrix $P$.

Proof. We refer the reader to Chapter 4 in [139] for the case $t=\sqrt{2}$. Now for any $s, t>0$ it is easy to see that the operator $U_{\frac{t}{s}}$ is bijective on $\mathcal{S}\left(\mathbb{R}^{n}\right)$. Using (4.2.2) we obtain $\beta_{t}\left[\mathcal{S}\left(\mathbb{R}^{n}\right)\right]=$ $\beta_{s}\left[\mathcal{S}\left(\mathbb{R}^{n}\right)\right]$. This shows that (4.2.4) holds true for all $t>0$.

Remark 4.2.1. The above proposition shows that if $f \in \beta_{t}\left[\mathcal{S}\left(\mathbb{R}^{n}\right)\right]$ then $z_{j}^{2} f(z) \in \beta_{t}\left[\mathcal{S}\left(\mathbb{R}^{n}\right)\right]$ for all $j=1, \cdots, n$. Hence by the reproducing kernel property of $H^{2}\left(\mathbb{C}^{n}\right)$ the following estimate holds true

$$
|f(z)| \prod_{j=1}^{n}\left(1+\left|z_{j}\right|^{2}\right)=\left|\left\langle f \prod_{j=1}^{n}\left(1+w_{j}^{2}\right), K_{z}\right\rangle\right| \leq\left\|f \prod_{j=1}^{n}\left(1+w_{j}^{2}\right)\right\|_{H^{2}\left(\mathbb{C}^{n}\right)} e^{\frac{|z|^{2}}{2}} .
$$

Via the push-forward under $\beta_{t}$ each operator $D$ on $H^{2}\left(\mathbb{C}^{n}\right)$ corresponds to the operator $\beta_{t}^{-1} D \beta_{t}$ on $L^{2}\left(\mathbb{R}^{n}\right)$. The next proposition exhibit the push-forward under $\beta_{t}$ of a family of integral operators acting on $\beta_{t}\left[\mathcal{S}\left(\mathbb{R}^{n}\right)\right] \subset H^{2}\left(\mathbb{C}^{n}\right)$.

Proposition 4.2.2. Let $\left(A_{s}\right)_{s \geq 0}$ be a family of integral operators acting on $\beta_{t}\left[\mathcal{S}\left(\mathbb{R}^{n}\right)\right]$. For each $s \geq 0$ we write $K(s, z, \bar{w})$ for the integral kernel of $A_{s}$ i.e.

$$
\left(A_{s} F\right)(z)=\int_{\mathbb{C}^{n}} K(s, z, \bar{w}) F(w) d \mu(w), \quad \text { for all } \quad F \in \beta_{t}\left[\mathcal{S}\left(\mathbb{R}^{n}\right)\right]
$$

Let $(x, y),(z, w)$ denote the coordinates in $\mathbb{R}^{n} \times \mathbb{R}^{n}, \mathbb{C}^{n} \times \mathbb{C}^{n}$ respectively. For a fixed $t>0$ we write $\beta_{t, x \rightarrow z}$ and $\beta_{t, y \rightarrow w}$ for the Bargmann transform on $L^{2}\left(\mathbb{R}^{n}\right)$ w.r.t the variables $x$ and $y$ respectively. Assume that $K(0, z, \bar{w})=e^{z \bar{w}}$ and for each $s>0$ we have

1. $K(s, z, \bar{w}) \in \beta_{t, x \rightarrow z}\left[\mathcal{S}\left(\mathbb{R}^{n}\right)\right], \quad$ for all $w \in \mathbb{C}^{n}$,

2. $\left[\beta_{t, x \rightarrow z}^{-1} K(s, z, w)\right](x) \in H^{2}\left(\mathbb{C}_{w}^{n}\right) \quad$ for all $\quad x \in \mathbb{R}^{n}$. 
Furthermore, we suppose that for each $x \in \mathbb{R}^{n}$ there is a constant $C_{x}$ depending on $x$ such that

$$
\int_{\mathbb{C}^{n}}\left|\Phi_{z}^{t}(x) K(s, z, \bar{w})\right| d \mu(z) \leq C_{x} e^{\frac{|w|^{2}}{2}}, \quad \text { for all } \quad s>0, w \in \mathbb{C}^{n}
$$

Then for each $s>0$ the corresponding operator $\beta_{t}^{-1} A_{s} \beta_{t}$ on $\mathcal{S}\left(\mathbb{R}^{n}\right)$ is an integral operator given by:

$$
\left[\beta_{t}^{-1} A_{s} \beta_{t} f\right](x)=\int_{\mathbb{R}^{n}} k(s, x, y) f(y) d y
$$

where

$$
k(s, x, y)=\beta_{t, y \rightarrow w}^{-1}\left[\beta_{t, x \rightarrow z}^{-1}[K(s, z, w)](x)\right](y) .
$$

Moreover, for each $x \in \mathbb{R}^{n}$ we have

$$
\lim _{s \rightarrow 0} \int_{\mathbb{R}^{n}} k(s, x, y) f(y) d y=\left[\beta_{t}^{-1} A_{0} \beta_{t} f\right](x)=f(x), \quad \text { for all } \quad f \in \mathcal{S}\left(\mathbb{R}^{n}\right) .
$$

Proof. Let $f \in \mathcal{S}\left(\mathbb{R}^{n}\right)$ and fix $t>0$. For each $s \geq 0$ we have

$$
A_{s}\left[\beta_{t} f\right](z)=\int_{\mathbb{C}^{n}} K(s, z, \bar{w})\left[\beta_{t} f\right](w) d \mu(w) .
$$

Hence for each $x \in \mathbb{R}^{n}$ we can write

$$
\begin{aligned}
{\left[\beta_{t}^{-1} A_{s} \beta_{t} f\right](x) } & =\int_{\mathbb{C}^{n}} \Phi_{z}^{t}(x) A_{s}\left[\beta_{t} f\right](z) d \mu(z) \\
& =\int_{\mathbb{C}^{n}} \Phi_{z}^{t}(x)\left[\int_{\mathbb{C}^{n}} K(s, z, \bar{w})\left[\beta_{t} f\right](w) d \mu(w)\right] d \mu(z) .
\end{aligned}
$$

Equations (4.2.5) and (4.2.6) allow us to apply Fubini theorem to the above integral. Indeed,

$$
\begin{aligned}
\int_{\mathbb{C}^{n}}\left|\left[\beta_{t} f\right](w)\right|\left[\int_{\mathbb{C}^{n}}\left|\Phi_{z}^{t}(x) K(s, z, \bar{w})\right| d \mu(z)\right] d \mu(w) \leq C_{x} \int_{\mathbb{C}^{n}}\left|\left[\beta_{t} f\right](w)\right| e^{\frac{|w|^{2}}{2}} d \mu(w) \\
\leq C_{x}\left\|\left[\beta_{t} f\right] \prod_{j=1}^{n}\left(1+w_{j}^{2}\right)\right\|_{H^{2}\left(\mathbb{C}^{n}\right)} \pi^{-n} \int_{\mathbb{C}^{n}} \prod_{j=1}^{n}\left(1+\left|w_{j}\right|^{2}\right)^{-1} d v(w)<\infty
\end{aligned}
$$

This shows that

$$
\begin{aligned}
{\left[\beta_{t}^{-1} A_{s} \beta_{t} f\right](x) } & =\int_{\mathbb{C}^{n}}\left[\beta_{t} f\right](w)\left[\int_{\mathbb{C}^{n}} K(s, z, \bar{w}) \Phi_{z}^{t}(x) d \mu(z)\right] d \mu(w) \\
& =\int_{\mathbb{C}^{n}}\left[\beta_{t} f\right](w)\left[\beta_{t, x \rightarrow z}^{-1} K(s, z, \bar{w})\right](x) d \mu(w) \\
& =\int_{\mathbb{R}^{n}} f(y) \beta_{t, y \rightarrow w}^{-1}\left[\beta_{t, x \rightarrow z}^{-1}[K(s, z, w)](x)\right](y) d y .
\end{aligned}
$$

Equation (4.2.8) follows from (4.2.9) by an application of the Lebesgue dominated convergence theorem together with the fact that $K(0, z, \bar{w})=e^{z \bar{w}}$. 
Remark 4.2.2. With the notation used in the above proposition we remark that if $f(x, y) \in$ $L^{2}\left(\mathbb{R}^{n} \times \mathbb{R}^{n}\right)$ and $\beta_{t, 2 n}$ denotes the $2 n$-dimensional Bargmann transform (i.e. on $L^{2}\left(\mathbb{R}^{2 n}\right)$ then

$$
\begin{aligned}
{\left[\beta_{t, 2 n} f\right](z, w) } & =\int_{\mathbb{R}^{n} \times \mathbb{R}^{n}} f(x, y) \Phi_{(\bar{z}, \bar{w})}^{t}(x, y) d x d y=\int_{\mathbb{R}^{n} \times \mathbb{R}^{n}} f(x, y) \Phi_{\bar{z}}^{t}(x) \Phi_{\bar{w}}^{t}(y) d x d y \\
& =\int_{\mathbb{R}^{n}} \Phi_{\bar{w}}^{t}(y) \int_{\mathbb{R}^{n}} f(x, y) \Phi_{\bar{z}}^{t}(x) d x d y=\int_{\mathbb{R}^{n}} \Phi_{\bar{w}}^{t}(y)\left[\beta_{t, x \rightarrow z} f(x, y)\right](z) d y \\
& =\beta_{t, y \rightarrow w}\left[\beta_{t, x \rightarrow z}[f(x, y)](z)\right](w)=\beta_{t, x \rightarrow z}\left[\beta_{t, y \rightarrow w}[f(x, y)](w)\right](z) .
\end{aligned}
$$

In the above equality we applied Fubini's theorem using the fact that $\Phi_{(\bar{z}, \bar{w})}^{t}(x, y) \in L^{2}\left(\mathbb{R}^{2 n}\right)$. Hence for any function $F \in H^{2}\left(\mathbb{C}^{2 n}\right)$ we have

$$
\left[\beta_{t, 2 n}^{-1} F(z, w)\right](x, y)=\left[\beta_{t, x \rightarrow z}^{-1}\left[\beta_{t, y \rightarrow w}^{-1} F(z, w)\right](y)\right](x)=\left[\beta_{t, y \rightarrow w}^{-1}\left[\beta_{t, x \rightarrow z}^{-1} F(z, w)\right](x)\right](y)
$$

Using this observation together with above proposition one can easily show that if $T$ is an integral operator on $L^{2}\left(\mathbb{R}^{n}\right)$ with a kernel $k(x, y) \in L^{2}\left(\mathbb{R}^{2 n}\right)$ then the corresponding operator $\beta_{t} T \beta_{t}^{-1}$ on $H^{2}\left(\mathbb{C}^{n}\right)$ is an integral operator given by:

$$
\beta_{t} T \beta_{t}^{-1} F(z)=\int_{\mathbb{C}^{n}} K(z, \bar{u}) F(u) d \mu(u)
$$

where $K$ is the (2n-dimensional) Bargmann transform of $k$ (c.f. Chapter 1 in [84] for the case $t=\sqrt{2})$.

For each $i \in\{1, \cdots, n\}$ let $x_{i}$ (respectively $\frac{\partial}{\partial x_{i}}$ ) be the operators of multiplication (respectively differentiation) with respect to the $\mathrm{i}$-th coordinate of $\mathbb{R}^{n}$. Under the Bargmann transform $\beta_{t}$ these differential operators correspond to the following Toeplitz operators densely defined on $H^{2}\left(\mathbb{C}^{n}\right)$ :

$$
\begin{aligned}
x_{i} & \longleftrightarrow \frac{1}{t} T_{z_{i}+\bar{z}_{i}} \\
\frac{\partial}{\partial x_{i}} & \longleftrightarrow \frac{t}{2} T_{z_{i}-\bar{z}_{i}}
\end{aligned}
$$

or equivalently

$$
\begin{aligned}
& T_{z_{i}} \longleftrightarrow \frac{t}{2} x_{i}-\frac{1}{t} \frac{\partial}{\partial x_{i}} \\
& T_{\bar{z}_{i}} \longleftrightarrow \frac{t}{2} x_{i}+\frac{1}{t} \frac{\partial}{\partial x_{i}}
\end{aligned}
$$

We only prove (4.2.13). Let $C_{0}^{\infty}\left(\mathbb{R}^{n}\right)$ denotes the space of all smooth functions with compact support in $\mathbb{R}^{n}$. Then for any $f \in C_{0}^{\infty}\left(\mathbb{R}^{n}\right)$ we have 


$$
\begin{aligned}
\beta_{t}\left(\frac{t}{2} x_{i}+\frac{1}{t} \frac{\partial}{\partial x_{i}}\right) f(z) & =\int_{\mathbb{R}^{n}}\left(\frac{t}{2} x_{i}+\frac{1}{t} \frac{\partial}{\partial x_{i}}\right) f(x) \Phi_{\bar{z}}^{t}(x) d x \\
& =\int_{\mathbb{R}^{n}} \frac{t}{2} x_{i} f(x) \Phi_{\bar{z}}^{t}(x) d x-\int_{\mathbb{R}^{n}}\left(z_{i}-\frac{t}{2} x_{i}\right) f(x) \Phi_{\bar{z}}^{t}(x) d x \\
& =\int_{\mathbb{R}^{n}}\left(t x_{i}-z_{i}\right) \Phi_{\bar{z}}^{t}(x) f(x) d x=\frac{\partial}{\partial z_{i}} \int_{\mathbb{R}^{n}} \Phi_{\bar{z}}^{t}(x) f(x) d x \\
& =\frac{\partial}{\partial z_{i}}\left[\beta_{t} f\right](z)=\left[T_{\bar{z}_{i}} \beta_{t} f\right](z) .
\end{aligned}
$$

In the following we aim to calculate

$$
\left[\beta_{t, y \rightarrow w}^{-1}\left[\beta_{t, x \rightarrow z}^{-1} e^{z A w}\right](x)\right](y),
$$

where $A \in M_{n}(\mathbb{C})$ is a Hermitian such that $A \bar{A}=\bar{A} A,\|\bar{A} A\|<1$ and the matrix $(I d+$ $A \bar{A})(I d-A \bar{A})^{-1}$ is positive definite. The reason for this calculation is motivated by the following observation: let $N \in \mathbb{N}$ be fixed and put $n=2 N$. If $\mathcal{L}$ is the operator defined on $L^{2}\left(\mathbb{R}^{n}\right)$ obtained by applying the partial Fourier transform to the sub-Laplacian on the $(2 N+1)$ dimensional Heisenberg group then the ,heat kernel” of its corresponding Toeplitz on $H^{2}\left(\mathbb{C}^{n}\right)$ (c.f. Definition 4.3.3) is of the form $e^{z A \bar{w}}$ where $(z, w) \in \mathbb{C}^{n} \times \mathbb{C}^{n}$ and $A$ has the previously mentioned properties. Hence by Proposition 4.2.2 we get the heat kernel of $\mathcal{L}$ by (4.2.14). The next proposition is essential in calculating (4.2.14) and its proof is given in Appendix A.3.

Proposition 4.2.3. Let $E \in M_{n}(\mathbb{C})$ be a Hermitian $n \times n$ complex matrix such that $\|E\|<\frac{1}{2}$. Denote by $F$ the real matrix $F:=E+\bar{E}$ and assume that $(I d-F)(I d+F)^{-1}$ is positive definite. We write $\beta_{t, x \rightarrow z}^{-1}$ for the n-dimensional inverse Bargmann transform on $H^{2}\left(\mathbb{C}^{n}\right)$. Then for fixed vector $u \in \mathbb{C}^{n}$ we have

$$
\beta_{t, x \rightarrow z}^{-1} \exp \{u \cdot z+z E z\}(x)=c \exp \left\{u(I d+F)^{-1} t x-\frac{t^{2}}{4} x(I d-F)(I d+F)^{-1} x\right\},
$$

where $c$ is the constant given by:

$$
\begin{aligned}
c=t^{\frac{n}{2}}(2 \pi)^{-\frac{n}{4}}\left(\frac{\operatorname{det}(I d-F)(I d+F)^{-1}}{\operatorname{det}(I d-4 E \bar{E})}\right)^{\frac{1}{4}} & \\
& \times \exp \frac{1}{2}\left\{u \bar{E}(I d-4 E \bar{E})^{-1} u+\bar{u}(I d-4 E \bar{E})^{-1} u+\bar{u} E(I d-4 E \bar{E})^{-1} \bar{u}\right. \\
& \left.\quad-u(I d+F)^{-1}(I d-F)^{-1} u-u(I d-F)^{-1}(I d+F)^{-1} \bar{u}\right\} .
\end{aligned}
$$

The next proposition gives the explicit expression of (4.2.14).

Proposition 4.2.4. Denote by $(x, y)$, $(z, w)$ the coordinates in $\mathbb{R}^{n} \times \mathbb{R}^{n}, \mathbb{C}^{n} \times \mathbb{C}^{n}$ respectively. Let $A \in M_{n}(\mathbb{C})$ be a Hermitian matrix and write $F:=-A \bar{A}$. Suppose that $A \bar{A}=\bar{A} A$, $\|A \bar{A}\|<1$ and the matrix $(I d-F)(I d+F)^{-1}$ is positive definite then 


$$
\begin{aligned}
& {\left[\beta_{t, x \rightarrow z}^{-1}\left[\beta_{t, y \rightarrow w}^{-1} e^{z A w}\right](y)\right](x)=} {\left[\beta_{t, y \rightarrow w}^{-1}\left[\beta_{t, x \rightarrow z}^{-1} e^{z A w}\right](x)\right](y) } \\
&=t^{n}(2 \pi)^{-\frac{n}{2}} \sqrt{\operatorname{det}(I d+F)^{-1}} \\
& \times \exp \left\{\frac { - t ^ { 2 } } { 4 } \left\{y(I d+F)^{-1}(I d-F) y\right.\right. \\
&\left.\left.+x(I d+F)^{-1}(I d-F) x\right\}+t^{2} y(I d+F)^{-1} A x\right\} .
\end{aligned}
$$

Proof. Using Equation (4.2.1) we obtain:

$$
\begin{aligned}
& {\left[\beta_{t, x \rightarrow z}^{-1}\left[\beta_{t, y \rightarrow w}^{-1} e^{z A w}\right](y)\right](x)=\left[\beta_{t, x \rightarrow z}^{-1}\left[\Phi_{A \bar{z}}^{t}(y)\right]\right](x) } \\
= & t^{\frac{n}{2}}(2 \pi)^{-\frac{n}{4}} e^{-\frac{t^{2}}{4} y^{2}}\left[\beta_{t, x \rightarrow z}^{-1} \exp \left\{t y A^{T} z-\frac{1}{2}\left(z^{T} A\right)\left(A^{T} z\right)\right\}\right](x) \\
= & t^{\frac{n}{2}}(2 \pi)^{-\frac{n}{4}} e^{-\frac{t^{2}}{4} y^{2}}\left[\beta_{t, x \rightarrow z}^{-1} \exp \left\{t(A y) \cdot z-z \frac{A \bar{A}}{2} z\right\}\right](x) .
\end{aligned}
$$

Applying Equations (4.2.15) and (4.2.16) for $u=t A y, E=\bar{E}=-\frac{A \bar{A}}{2}$ and $F=-A \bar{A}$ we get:

$$
\left[\beta_{t, x \rightarrow z}^{-1} \exp \left\{t y \bar{A} z-z \frac{A \bar{A}}{2} z\right\}\right](x)=c \exp \left\{t^{2} y(I d+F)^{-1} A x-\frac{t^{2}}{4} x(I d+F)^{-1}(I d-F) x\right\}
$$

where

$$
\begin{aligned}
& c= t^{\frac{n}{2}}(2 \pi)^{-\frac{n}{4}}\left(\frac{\operatorname{det}(I d-F)(I d+F)^{-1}}{\operatorname{det}(I d-4 E \bar{E})}\right)^{\frac{1}{4}} \\
& \times \exp \frac{1}{2}\left\{u \bar{E}(I d-4 E \bar{E})^{-1} u^{T}+\bar{u}(I d-4 E \bar{E})^{-1} u^{T}+\bar{u} E(I d-4 E \bar{E})^{-1} u^{\star}\right. \\
&\left.\quad \quad-u(I d+F)^{-1}(I d-F)^{-1} u^{T}-u(I d-F)^{-1}(I d+F)^{-1} u^{\star}\right\} \\
& \quad=t^{\frac{n}{2}}(2 \pi)^{-\frac{n}{4}} \sqrt{\operatorname{det}(I d+F)^{-1}} \exp \frac{-t^{2}}{4}\left\{y\left(I d-A^{2} \bar{A}^{2}\right)^{-1}\left(2 A^{2} \bar{A}^{2}+2 \bar{A} A\right) y\right\} .
\end{aligned}
$$

Substituting (4.2.19) and (4.2.20) into (4.2.18) we then obtain:

$$
\begin{aligned}
& {\left[\beta_{t, x \rightarrow z}^{-1}\left[\beta_{t, y \rightarrow w}^{-1} e^{z A w}\right](y)\right](x)} \\
& =t^{n}(2 \pi)^{-\frac{n}{2}} \sqrt{\operatorname{det}(I d+F)^{-1}} \exp \left\{\frac{-t^{2}}{4}\left\{y\left(I d-A^{2} \bar{A}^{2}\right)^{-1}\left(2 A^{2} \bar{A}^{2}+2 \bar{A} A+I d-A^{2} \bar{A}^{2}\right) y\right\}\right. \\
& \left.+t^{2} y(I d+F)^{-1} A x-\frac{t^{2}}{4} x(I d+F)^{-1}(I d-F) x\right\} \\
& =t^{n}(2 \pi)^{-\frac{n}{2}} \sqrt{\operatorname{det}(I d+F)^{-1}} \exp \left\{\frac { - t ^ { 2 } } { 4 } \left\{y(I d+F)^{-1}(I d-F) y\right.\right. \\
& \left.\left.-x(I d+F)^{-1}(I d-F) x\right\}+t^{2} y(I d+F)^{-1} A x\right\} .
\end{aligned}
$$


Using a similar calculation together with the fact that $y(I d+F)^{-1} A x=y A(I d+F)^{-1} x$ for all $(x, y) \in \mathbb{R}^{n} \times \mathbb{R}^{n}$ one can show that

$$
\left[\beta_{t, y \rightarrow w}^{-1}\left[\beta_{t, x \rightarrow z}^{-1} e^{z A w}\right](x)\right](y)
$$

is also given by (4.2.17).

Remark 4.2.3. Let $A \in M_{n}(\mathbb{C})$ be a Hermitian matrix and denote by $\rho(A) \geq 0$ its spectral radius. Then the function $e^{z A w} \in H^{2}\left(\mathbb{C}^{2 n}\right)$ if and only if $\rho(A)<1$. Indeed, since $A$ is Hermitian there is a unitary matrix $P$ such that

$$
A=P D\left(\lambda_{1}, \cdots, \lambda_{n}\right) P^{-1}
$$

where $D\left(\lambda_{1}, \cdots, \lambda_{n}\right)$ is the diagonal matrix whose entries $\lambda_{j}$ are the eigenvalues of $A\left(\lambda_{j}\right.$ are real). Since the Segal-Bargmann space is invariant under a unitary transformation of coordinates it follows that $e^{z A w} \in H^{2}\left(\mathbb{C}^{2 n}\right)$ if and only if $e^{\sum \lambda_{j} z_{j} w_{j}} \in H^{2}\left(\mathbb{C}^{2 n}\right)$ (here we used the transformation $(z, w) \longmapsto(\bar{P} z, P w))$. This is equivalent to say that for each $j=1, \cdots, n$

$$
e^{\lambda_{j} z_{j} w_{j}}=\sum_{\alpha \in \mathbb{N}_{0}} \lambda_{j}^{\alpha} \frac{\left(z_{j} w_{j}\right)^{\alpha}}{\alpha !} \in H^{2}\left(\mathbb{C}^{2}\right) \Longleftrightarrow \sum_{\alpha \in \mathbb{N}_{0}}\left|\lambda_{j}\right|^{2 \alpha}<\infty \Longleftrightarrow\left|\lambda_{j}\right|<1 .
$$

Hence by Remark 4.2.2 it follows that if $A \in M_{n}(\mathbb{C})$ is such that $\rho(A)<1$ and satisfies the conditions in the above proposition then the $2 n$-dimensional inverse Bargmann transform $\beta_{t, 2 n}^{-1} e^{z A w} \in L^{2}\left(\mathbb{R}^{2 n}\right)$ exists and is given by (4.2.17).

The next example is an application of Proposition 4.2.2 for a certain class of integral operators. This example will be essential for obtaining the heat kernel of a class of elliptic operators from the „heat kernel” of their corresponding Toeplitz operators (c.f. Corollary 4.3.2).

Example 4.2.1. Let $A \in M_{n}(\mathbb{C})$ be a positive semidefinite matrix such that $(A+\bar{A})$ is positive definite and $A \bar{A}=\bar{A} A$. We consider a family $\left(T_{s}\right)_{s \geq 0}$ of integral operators acting on $\beta_{t}\left[\mathcal{S}\left(\mathbb{R}^{n}\right)\right] \subset H^{2}\left(\mathbb{C}^{n}\right)$ defined by

$$
\left(T_{s} F\right)(z):=\int_{\mathbb{C}^{n}} e^{z e^{-s A} \bar{w}} F(w) d \mu(w), \quad \text { for all } \quad F \in \beta_{t}\left[\mathcal{S}\left(\mathbb{R}^{n}\right)\right] .
$$

Then for each fixed couple $s, t>0$ the corresponding operator $\beta_{t}^{-1} T_{s} \beta_{t}$ on $\mathcal{S}\left(\mathbb{R}^{n}\right)$ is an integral operator given by:

$$
\left[\beta_{t}^{-1} T_{s} \beta_{t} f\right](x)=\int_{\mathbb{R}^{n}} k(s, x, y) f(y) d y,
$$

where $k(s, x, y)$ is given by an application of (4.2.17) to the matrix $e^{-s A}$. Moreover, for each $x \in \mathbb{R}^{n}$ we have

$$
\lim _{s \rightarrow 0} \int_{\mathbb{R}^{n}} k(s, x, y) f(y) d y=\left[\beta_{t}^{-1} T_{0} \beta_{t} f\right](x)=f(x), \quad \text { for all } \quad f \in \mathcal{S}\left(\mathbb{R}^{n}\right) .
$$

Indeed, this is an application of Proposition 4.2.2 to the operators defined by the kernels $K(s, z, \bar{w})=e^{z e^{-s A} \bar{w}}$ together with another application of Proposition 4.2.4 to the matrix $e^{-s A}$. 
First we check condition (4.2.6) in Proposition 4.2.2. Since $(A+\bar{A})$ is positive definite we obtain the following estimate

$$
\begin{aligned}
\int_{\mathbb{C}^{n}}\left|\Phi_{z}^{t}(x) e^{z e^{-s A} \bar{w}}\right| d \mu(z)= & t^{\frac{n}{2}}(2 \pi)^{-\frac{n}{4}} e^{-t^{2} \frac{x^{2}}{4}} \\
& \times \int_{\mathbb{C}^{n}} \exp \left\{\frac{1}{2}\left(t x \bar{z}+t x z-\operatorname{Re} z^{2}+z e^{-s A} \bar{w}+\bar{z} e^{-s \bar{A}} w\right)\right\} d \mu(z) \\
\leq & C_{x} \int_{\mathbb{C}^{n}} e^{\left(e^{-s \bar{A}} w+t x\right) \cdot \overline{\bar{z}}} e^{\left(e^{-s A} \bar{w}+t x\right) \cdot \frac{z}{2}} e^{-\frac{|z|^{2}}{2}} d v(z) \\
= & (2 \pi)^{n} C_{x} \exp \left\{\frac{1}{2}\left(e^{-s A} \bar{w}+t x\right) \cdot\left(e^{-s \bar{A}} w+t x\right)\right\} \leq C_{x}^{\prime} e^{\frac{|w|^{2}}{2}}
\end{aligned}
$$

where $C_{x}$ and $C_{x}^{\prime}$ are constants depending on $x$. It remains to check the conditions in Proposition 4.2.4 for the matrix $e^{-s A}$. In fact, since $A$ and $\bar{A}$ commute it follows that $e^{-s A} e^{-s \bar{A}}=$ $e^{-s(A+\bar{A})}$. Moreover, since $(A+\bar{A})$ is positive definite we have $\left\|e^{-s(A+\bar{A})}\right\|<1$ and $(\mathrm{Id}-$ $\left.e^{-s(A+\bar{A})}\right)$ is positive definite.

At the end of this section we introduce the sub-Laplacian on the $(2 N+1)$-dimension Heisenberg group.

For a fixed $N \in \mathbb{N}$, let $\mathbb{H}_{(2 N+1)} \cong \mathbb{R}^{2 N} \times \mathbb{R}$ then $\mathbb{H}_{(2 N+1)}$ becomes a non-commutative Lie group when equipped with the product $\circ: \mathbb{R}^{2 N+1} \times \mathbb{R}^{2 N+1} \longrightarrow \mathbb{R}^{2 N+1}$ given by the formula:

$$
(x, u) \circ(y, \tilde{u}):=\left(x+y, u+\tilde{u}+\sum_{j=1}^{N}\left(x_{j} y_{N+j}-x_{N+j} y_{j}\right)\right),
$$

where $(x, u)=\left(x_{1}, \cdots, x_{2 N}, u\right),(y, \tilde{u})=\left(y_{1}, \cdots, y_{2 N}, \tilde{u}\right) \in \mathbb{R}^{2 N+1}$. This Lie group is called the $(2 N+1)$-dimensional Heisenberg group. If we denote by $\mathfrak{h}_{2 N+1}$ the corresponding Lie algebra of left invariant vector fields on $\mathbb{H}_{(2 N+1)}$ then a basis for $\mathfrak{h}_{2 N+1}$ is given by

$$
X_{l}=\frac{\partial}{\partial x_{l}}-x_{l+N} \frac{\partial}{\partial u}, \quad X_{l+N}=\frac{\partial}{\partial x_{l+N}}+x_{l} \frac{\partial}{\partial u} \quad \text { and } U=\frac{\partial}{\partial u} \quad \text { where } l=1, \cdots, N
$$

An easy computation shows that the above vector fields obey the relations $\left[X_{l}, X_{l+N}\right]=2 U$ and $\left[X_{l}, U\right]=\left[X_{l+N}, U\right]=0$ turning $\mathbb{H}_{(2 N+1)}$ into a 2-step nilpotent Lie group [174]. The vector fields $X_{l}$ and $X_{l+N}$ are called the Heisenberg vector fields and the sub-elliptic operator (c.f. [107])

$$
\Delta_{\text {sub }}:=-\frac{1}{2} \sum_{l=1}^{N}\left(X_{l}^{2}+X_{l+N}^{2}\right)
$$

is called the sub-Laplacian or the Heisenberg sub-Laplacian on $\mathbb{H}_{(2 N+1)}$. For more details on the analysis of the sub-Laplacian on the Heisenberg group we refere the reader to the book of S. Thangavelu ([165]).

\subsection{Heat kernel by Toeplitz operator theory techniques}

In this section, we give a new method for calculating the heat kernel of a certain type of subelliptic positive essentially selfadjoint differential operators by using Toeplitz operator theory 
techniques. Our approach relies on transforming (via the Bargmann transform) a differential operator with polynomial coefficients into a Toeplitz operator with a polynomial symbol acting on the Segal-Bargmann space. For suitable symbols, we are able to obtain the ,heat kernel” of the Toeplitz operator using Berezin's result on the exponential of a selfadjoint Toeplitz operator. The heat kernel of the differential operator is then obtained explicitly via an application of the inverse Bargmann transform to the ,heat kernel” of the corresponding Toeplitz operator. As an application, we determine the heat kernel of the Hermite operator on $\mathbb{R}^{n}$ as well as that of the isotropic twisted Laplacian on $\mathbb{R}^{n}$ (here $n=2 N$ with $N \in \mathbb{N}$ is arbitrary). We use the Fourier transform method to reduce the problem of finding the heat kernel of a certain family of subelliptic operators to a family of elliptic operators depending on a smaller number of variables and then apply the above mentioned technique. As a consequence, we obtain an explicit integral formula for the heat kernel of the Grusin operator on $\mathbb{R}^{n+1}$ as well as that of the sub-Laplace operator on $\mathbb{H}_{(2 N+1)}$. We shall start by recalling some basic definitions and introducing the method of Fourier transform.

Definition 4.3.1. Let $\mathcal{L}$ be a differential operator defined on $\mathbb{R}^{n}$ then the operator

$$
\mathcal{P}:=\frac{\partial}{\partial t}+\mathcal{L}
$$

defined on $C^{\infty}\left(\mathbb{R}_{+} \times \mathbb{R}^{n}\right)$ is called the heat operator with respect to $\mathcal{L}$ on $\mathbb{R}^{n}$.

Definition 4.3.2. A fundamental solution $K(t, x, y) \in C^{\infty}\left(\mathbb{R}_{+} \times \mathbb{R}^{n} \times \mathbb{R}^{n}\right)$ of the operator $\mathcal{P}$ (in case of existence) is called the heat kernel i.e. it satisfies the heat equation:

$$
\begin{cases}\mathcal{P}(K(t, \cdot, y))=0, & \forall t>0, y \in \mathbb{R}^{n} ; \\ \lim _{t \downarrow 0} K(t, x, \cdot)=\delta_{x}, \quad \forall x \in \mathbb{R}^{n}\end{cases}
$$

where $\delta_{x}$ is the Dirac distribution and the above limit is considered in the distributional sense i.e.

$$
\lim _{t \downarrow 0} \int_{\mathbb{R}^{n}} K(t, x, y) f(y) d y=f(x) \quad \text { for all } f \in C_{0}^{\infty}\left(\mathbb{R}^{n}\right) .
$$

Let us explain now the method of the Fourier transform. Denote by $(x, u) \in \mathbb{R}^{n} \times \mathbb{R}$ the coordinates on $\mathbb{R}^{n+1}$. We are interested in second order differential operators with coefficient that are independent of the $u$-variable. Consider the partial Fourier transformation in the $u$ variable $\mathcal{F}_{u}: L^{2}\left(\mathbb{R}^{n+1}\right) \longrightarrow L^{2}\left(\mathbb{R}^{n+1}\right)$ given by

$$
\left(\mathcal{F}_{u} f\right)(x, \xi):=\frac{1}{\sqrt{2 \pi}} \int_{\mathbb{R}} f(x, u) e^{-i u \xi} d u, \quad \text { for any } f \in L^{2}\left(\mathbb{R}^{n+1}\right) .
$$

The importance of the partial Fourier transform in reducing the problem of obtaining the heat kernel for some differential operators is given in the following proposition.

Proposition 4.3.1. Let $\mathcal{L}$ be a differential operator acting on the Schwartz space $\mathcal{S}\left(\mathbb{R}^{n+1}\right)$ and having the following form:

$$
\mathcal{L}=\sum_{k=1}^{n}\left\{a_{k} \frac{\partial^{2}}{\partial x_{k}^{2}}+\sum_{j=1}^{n} b_{k, j} \frac{\partial^{2}}{\partial x_{k} \partial x_{j}}+c_{k} \frac{\partial}{\partial x_{k}}+d_{k} \frac{\partial^{2}}{\partial x_{k} \partial u}\right\}+e \frac{\partial}{\partial u}+f \frac{\partial^{2}}{\partial u^{2}},
$$

where $a_{k}, b_{k, j}, c_{k}, d_{k}, e, f$ are (smooth) functions of $x \in \mathbb{R}^{n}$. Then the following holds: 
1. Applying the inverse Fourier transformation on $\mathcal{S}\left(\mathbb{R}^{n+1}\right)$ we get:

$$
\begin{aligned}
\mathcal{L}_{\xi}: & =\mathcal{F}_{u} \mathcal{L} \mathcal{F}_{u \mid(, \xi)}^{-1} \\
& =\sum_{k}\left\{a_{k} \frac{\partial^{2}}{\partial x_{k}^{2}}+\sum_{j} b_{k, j} \frac{\partial^{2}}{\partial x_{k} \partial x_{j}}+\left(c_{k}+i \xi d_{k}\right) \frac{\partial}{\partial x_{k}}\right\}+i \xi e-f \xi^{2} .
\end{aligned}
$$

2. For each $\xi \in \mathbb{R}$ assume that the heat kernel of the operator $\mathcal{L}_{\xi}$ on $\mathbb{R}^{n}$ exists and denote it by $K_{\xi}(t, x, \tilde{x})$. Suppose that for every $t>0$ the function $K(t, x, \tilde{x}, \xi):=K_{\xi}(t, x, \tilde{x}) \in$ $C^{\infty}\left(\mathbb{R}_{x}^{n} \times \mathbb{R}_{\tilde{x}}^{n} \times \mathbb{R}_{\xi}\right)$ such that $K(t, x, \tilde{x}, \cdot) \in L^{1}(\mathbb{R})$ and satisfies the following conditions

(i) For each fixed pair $(t, \tilde{x})$, multi-index $\alpha \in \mathbb{N}_{0}^{n}$ with $|\alpha| \in\{1,2\}$ and each $j=$ $1, \cdots, n$ there are integrable functions $g_{0}, g_{1}$ and $g_{2}$ on $\mathbb{R}$ such that

$$
\begin{aligned}
& \left|\frac{\partial^{|\alpha|}}{\partial x^{\alpha}} K(t, x, \tilde{x}, \xi)\right| \leq g_{0}(\xi) \in L^{1}(\mathbb{R}), \\
& \left|\xi^{|\alpha|} K(t, x, \tilde{x}, \xi)\right| \leq g_{1}(\xi) \in L^{1}(\mathbb{R}), \quad \text { and } \\
& \left|\xi \frac{\partial}{\partial x_{j}} K(t, x, \tilde{x}, \xi)\right| \leq g_{2}(\xi) \in L^{1}(\mathbb{R}), \quad \text { for all } x \in \mathbb{R}^{n} .
\end{aligned}
$$

(ii) For each fixed triple $\left(t_{0}, x, \tilde{x}\right)$ there exist a positive number $\epsilon<t_{0}$ and an integrable function $g_{3}$ depending on $\epsilon$ such that

$$
\left|\frac{\partial}{\partial t} K(t, x, \tilde{x}, \xi)\right| \leq g_{3}(\xi) \in L^{1}(\mathbb{R}), \quad \text { for all } \quad t \in\left[t_{0}-\epsilon, t_{0}+\epsilon\right] .
$$

(iii) For every fixed $x \in \mathbb{R}^{n}$ and each function $h \in \mathcal{S}\left(\mathbb{R}^{n+1}\right)$ there is a function $g \in$ $L^{1}(\mathbb{R})$ depending on $x$ and h only such that

$$
\left|\int_{\mathbb{R}^{n}} K_{\xi}(t, x, \tilde{x}) h(\tilde{x}, \xi) d \tilde{x}\right| \leq g(\xi) \quad \text { for all } t>0
$$

Then the heat kernel of the operator $\mathcal{L}$ on $\mathbb{R}^{n+1}$ is given by:

$$
K(t, x, u, \tilde{x}, \tilde{u})=\frac{1}{2 \pi} \int_{\mathbb{R}} e^{i \xi(u-\tilde{u})} K_{\xi}(t, x, \tilde{x}) d \xi
$$

Proof. 1. Eq. (4.3.2) follows from the following well known identities on $\mathcal{S}\left(\mathbb{R}^{n+1}\right)$

$$
\mathcal{F}_{u \rightarrow \xi}\left(\frac{\partial^{l}}{\partial u^{l}} h\right)(x, \xi)=(i \xi)^{l} \mathcal{F}_{u \rightarrow \xi} h(x, \xi), \text { and } \quad \mathcal{F}_{u \rightarrow \xi}\left(\frac{\partial^{\gamma}}{\partial x^{\gamma}} h\right)(x, \xi)=\frac{\partial^{\gamma}}{\partial x^{\gamma}}\left(\mathcal{F}_{u \rightarrow \xi} h\right)(x, \xi)
$$

for all $h \in \mathcal{S}\left(\mathbb{R}^{n+1}\right), l \in \mathbb{N}_{0}$ and all multi-indices $\gamma \in \mathbb{N}_{0}^{n}$.

2. As for the second assertion we have: 
(a) The conditions (i) and (ii) on the heat kernel $K_{\xi}(t, x, \tilde{x})$ stated above allows us to use the Lebesgue theorem and apply the operator $\left(\frac{\partial}{\partial t}+\mathcal{L}\right)$ under the integral sign as follows

$$
\begin{aligned}
\left(\frac{\partial}{\partial t}\right. & +\mathcal{L}) \int e^{i \xi(u-\tilde{u})} K_{\xi}(t, x, \tilde{x}) d \xi=\int e^{i \xi(u-\tilde{u})} \frac{\partial K_{\xi}(t, x, \tilde{x})}{\partial t} d \xi \\
& +\int \mathcal{L} e^{i \xi(u-\tilde{u})} K_{\xi}(t, x, \tilde{x}) d \xi=\int e^{i \xi(u-\tilde{u})} \frac{\partial K_{\xi}(t, x, \tilde{x})}{\partial t} d \xi \\
& +\int e^{i \xi(u-\tilde{u})}\left(\sum_{k} a_{k} \frac{\partial^{2}}{\partial x_{k}^{2}}+\sum_{j} b_{k, j} \frac{\partial^{2}}{\partial x_{k} \partial x_{j}}+c_{k} \frac{\partial}{\partial x_{k}}\right) K_{\xi}(t, x, \tilde{x}) d \xi \\
& +\int\left\{\sum_{k} d_{k} \frac{\partial^{2}}{\partial u \partial x_{k}}+e \frac{\partial}{\partial u}+f \frac{\partial^{2}}{\partial u^{2}}\right\} e^{i \xi(u-\tilde{u})} K_{\xi}(t, x, \tilde{x}) d \xi \\
& =\int e^{i \xi(u-\tilde{u})}\left(\frac{\partial}{\partial t}+\mathcal{L}_{\xi}\right) K_{\xi}(t, x, \tilde{x}) d \xi=0 .
\end{aligned}
$$

(b) First let us note that for any $h \in C_{0}^{\infty}\left(\mathbb{R}^{n+1}\right)$ and any fixed $(x, \xi) \in \mathbb{R}^{n} \times \mathbb{R}$ we have

$$
\lim _{t \downarrow 0} \int_{\mathbb{R}^{n}} K_{\xi}(t, x, \tilde{x})\left(\mathcal{F}_{u \rightarrow \xi} h\right)(\tilde{x}, \xi) d \tilde{x}=\left(\mathcal{F}_{u \rightarrow \xi} h\right)(x, \xi) \quad \text { for all } h \in C_{0}^{\infty}\left(\mathbb{R}^{n+1}\right) .
$$

The above equation holds true since $\left(\mathcal{F}_{u \rightarrow \xi} h\right)(\tilde{x}, \xi) \in C_{0}^{\infty}\left(\mathbb{R}^{n}\right)$. Now let $(x, u) \in$ $\mathbb{R}^{n} \times \mathbb{R}$ be fixed. Using (iii) and by the Lebesgue dominated convergence theorem we can write

$$
\begin{aligned}
& \lim _{t \downarrow 0} \int_{\mathbb{R}^{n+1}} h(\tilde{x}, \tilde{u}) \int_{\mathbb{R}} e^{i \xi(u-\tilde{u})} K_{\xi}(t, x, \tilde{x}) d \xi d \tilde{x} d \tilde{u} \\
& =\lim _{t \downarrow 0} \int h(\tilde{x}, \tilde{u}) e^{-i \xi \tilde{u}} d \tilde{u} \cdot e^{i \xi u} K_{\xi}(t, x, \tilde{x}) d \xi d \tilde{x} \\
& =\lim _{t \downarrow 0} \sqrt{2 \pi} \int e^{i \xi u} \int K_{\xi}(t, x, \tilde{x})\left(\mathcal{F}_{u \rightarrow \xi} h\right)(\tilde{x}, \xi) d \tilde{x} d \xi \\
& =\sqrt{2 \pi} \int e^{i \xi u}\left(\mathcal{F}_{u \rightarrow \xi} h\right)(x, \xi) d \xi=(2 \pi) h(x, u) .
\end{aligned}
$$

Let $A=\left(a_{j k}\right) \in M_{n}(\mathbb{C})$ be a $n \times n$ positive semidefinite matrix such that $(A+\bar{A})$ is positive definite and $A \bar{A}=\bar{A} A$ (here $\bar{A}$ is the complex conjugate of $A$, i.e. $\bar{A}=\left(\overline{a_{j k}}\right) \in M_{n}(\mathbb{C})$ ). We consider the following differential operator on $\mathbb{R}^{n+1}$

$$
\begin{aligned}
\mathcal{L}:=-\sum a_{j j}\left(\frac{\partial^{2}}{\partial x_{j}^{2}}+x_{j}^{2} \frac{\partial^{2}}{\partial u^{2}}\right)-2 \operatorname{Re} \sum_{j>k} a_{j k} x_{j} x_{k} \frac{\partial^{2}}{\partial u^{2}}-2 \operatorname{Im} \sum_{j>k} a_{j k}\left(x_{k} \frac{\partial^{2}}{\partial x_{j} \partial u}-x_{j} \frac{\partial^{2}}{\partial x_{k} \partial u}\right) \\
-2 \operatorname{Re} \sum_{j>k} a_{j k} \frac{\partial^{2}}{\partial x_{k} \partial x_{j}} .
\end{aligned}
$$

It turns out that the above operator is subelliptic positive and essentially selfadjoint as an operator on the Schwartz space $\mathcal{S}\left(\mathbb{R}^{n+1}\right)$ (c.f. Theorems 4.3.1 and 4.3.7). Our goal is to give 
an explicit integral formula for the heat kernel of this operator under certain conditions. As an application, we obtain the heat kernel of the Grusin and sub-Laplace operators.

In his famous paper [107], L. Hörmander proved that if $\left\{X_{1}, \cdots, X_{m}\right\}$ are first order real vector fields over $\mathbb{R}^{n}$ with smooth coefficients such that the Lie algebra generated by these vector fields generates the tangent space of $\mathbb{R}^{n}$ in each point then the operator $E=\sum_{j=1}^{m} X_{j}^{2}$ satisfies a classical subelliptic estimate. This generating condition on the vector fields is usually known as the „bracket generating condition” or „Hörmander condition for hypo-ellipticity” (recall that every sub-elliptic operator is hypoelliptic [73]). The next theorem shows that the operator (4.3.6) is written as the sum of squares of first order real vector fields that satisfy the bracket generating condition and hence ensure the sub-ellipticity of the operator $\mathcal{L}$.

Theorem 4.3.1. Let $A \in M_{n}(\mathbb{C})$ be a $n \times n$ positive semidefinite matrix such that $(A+\bar{A})$ is positive definite. Consider the differential operator $\mathcal{L}$ on $\mathbb{R}^{n+1}$ defined by

$$
\begin{gathered}
\mathcal{L}=-\sum a_{j j}\left(\frac{\partial^{2}}{\partial x_{j}^{2}}+x_{j}^{2} \frac{\partial^{2}}{\partial u^{2}}\right)-2 \operatorname{Re} \sum_{j>k} a_{j k} x_{j} x_{k} \frac{\partial^{2}}{\partial u^{2}}-2 \operatorname{Im} \sum_{j>k} a_{j k}\left(x_{k} \frac{\partial^{2}}{\partial x_{j} \partial u}-x_{j} \frac{\partial^{2}}{\partial x_{k} \partial u}\right) \\
-2 \operatorname{Re} \sum_{j>k} a_{j k} \frac{\partial^{2}}{\partial x_{k} \partial x_{j}},
\end{gathered}
$$

where $(x, u)=\left(x_{1}, \cdots, x_{n}, u\right) \in \mathbb{R}^{n} \times \mathbb{R}$. Then $\mathcal{L}$ is a sub-elliptic, symmetric and positive operator on the Schwartz space $\mathcal{S}\left(\mathbb{R}^{n+1}\right)$.

Proof. We start by expressing $-\mathcal{L}$ as a sum of squares of real valued vector fields. We then investigate the bracket generating condition for the sub-ellipticity condition of the operator. Since $A$ is Hermitian it is easy to check that (4.3.7) can be written in the following form

$$
\begin{aligned}
\mathcal{L}=-\sum a_{j j}\left(\frac{\partial^{2}}{\partial x_{j}^{2}}+x_{j}^{2} \frac{\partial^{2}}{\partial u^{2}}\right)-\sum_{j \neq k} a_{j k} x_{j} x_{k} \frac{\partial^{2}}{\partial u^{2}} & -i \sum_{j \neq k} a_{j k}\left(x_{j} \frac{\partial^{2}}{\partial x_{k} \partial u}-x_{k} \frac{\partial^{2}}{\partial x_{j} \partial u}\right) \\
& -\sum_{j \neq k} a_{j k} \frac{\partial^{2}}{\partial x_{k} \partial x_{j}} .
\end{aligned}
$$

For each $j=1, \cdots, n$ consider the complex valued vector field $Z_{j}:=\frac{\partial}{\partial x_{j}}+i x_{j} \frac{\partial}{\partial u}$ and put $Z=\left(Z_{1}, \cdots, Z_{n}\right)$. Denote by $Z^{\star}$ the conjugate transpose of the row matrix $Z$ then

$$
\begin{aligned}
Z A Z^{\star}= & \sum a_{j j} Z_{j} \overline{Z_{j}}+\sum_{j \neq k} a_{j k} Z_{j} \overline{Z_{k}} \\
= & \sum a_{j j}\left(\frac{\partial^{2}}{\partial x_{j}^{2}}+x_{j}^{2} \frac{\partial^{2}}{\partial u^{2}}-i \frac{\partial}{\partial u}+i x_{j} \frac{\partial^{2}}{\partial x_{j} \partial u}-i x_{j} \frac{\partial^{2}}{\partial x_{j} \partial u}\right) \\
& +\sum_{j \neq k} a_{j k}\left(\frac{\partial^{2}}{\partial x_{k} \partial x_{j}}-i x_{k} \frac{\partial^{2}}{\partial x_{j} \partial u}+i x_{j} \frac{\partial^{2}}{\partial x_{k} \partial u}+x_{j} x_{k} \frac{\partial^{2}}{\partial u^{2}}\right) .
\end{aligned}
$$

Which shows that

$$
\mathcal{L}=-Z A Z^{\star}-i \operatorname{tr}(A) \frac{\partial}{\partial u}
$$


We use the above equation together with the fact that $A$ is positive semidefinite to write $-\mathcal{L}$ as a sum of squares of real valued vector fields. Since $A$ is positive semidefinite there exists a unique Hermitian positive semidefinite matrix $C=\left(c_{j k}\right)$ such that $A=C^{2}$ (c.f. Theorem 7.2.6 in [109]). Let us write $C=\alpha+i \beta$ where $\alpha=\left(\alpha_{j k}\right)$ (respectively $\beta=\left(\beta_{j k}\right)$ ) denotes the real symmetric (respectively antisymmetric) part of $C$. According to (4.3.8) we can write

$$
\mathcal{L}+i \operatorname{tr}(A) \frac{\partial}{\partial u}=-Z A Z^{\star}=-Z C C^{\star} Z^{\star}=-Z C(Z C)^{\star}=-\sum_{k}(Z C)_{k} \overline{(Z C)_{k}} .
$$

We calculate each candidate $(Z C)_{k} \overline{(Z C)_{k}}$ explicitly

$$
(Z C)_{k}=\sum_{j}\left(\alpha_{j k}+i \beta_{j k}\right)\left(\frac{\partial}{\partial x_{j}}+i x_{j} \frac{\partial}{\partial u}\right)=\sum_{j}\left(\alpha_{j k} \frac{\partial}{\partial x_{j}}-\beta_{j k} x_{j} \frac{\partial}{\partial u}\right)+i \sum_{j}\left(\alpha_{j k} x_{j} \frac{\partial}{\partial u}+\beta_{j k} \frac{\partial}{\partial x_{j}}\right) .
$$

Therefore

$$
\begin{aligned}
(Z C)_{k} \overline{(Z C)_{k}}= & {\left[\sum_{j}\left(\alpha_{j k} \frac{\partial}{\partial x_{j}}-\beta_{j k} x_{j} \frac{\partial}{\partial u}\right)+i \sum_{j}\left(\alpha_{j k} x_{j} \frac{\partial}{\partial u}+\beta_{j k} \frac{\partial}{\partial x_{j}}\right)\right] } \\
& \times\left[\sum_{j}\left(\alpha_{j k} \frac{\partial}{\partial x_{j}}-\beta_{j k} x_{j} \frac{\partial}{\partial u}\right)-i \sum_{j}\left(\alpha_{j k} x_{j} \frac{\partial}{\partial u}+\beta_{j k} \frac{\partial}{\partial x_{j}}\right)\right] \\
= & {\left[\sum_{j}\left(\alpha_{j k} \frac{\partial}{\partial x_{j}}-\beta_{j k} x_{j} \frac{\partial}{\partial u}\right)\right]^{2}+\left[\sum_{j}\left(\alpha_{j k} x_{j} \frac{\partial}{\partial u}+\beta_{j k} \frac{\partial}{\partial x_{j}}\right)\right]^{2} } \\
& +i \sum_{j, l}\left(\alpha_{j k} x_{j} \frac{\partial}{\partial u}+\beta_{j k} \frac{\partial}{\partial x_{j}}\right)\left(\alpha_{l k} \frac{\partial}{\partial x_{l}}-\beta_{l k} x_{l} \frac{\partial}{\partial u}\right) \\
& -i \sum_{j, l}\left(\alpha_{j k} \frac{\partial}{\partial x_{j}}-\beta_{j k} x_{j} \frac{\partial}{\partial u}\right)\left(\alpha_{l k} x_{l} \frac{\partial}{\partial u}+\beta_{l k} \frac{\partial}{\partial x_{l}}\right) .
\end{aligned}
$$

The imaginary part in the above equation reduces to $-a_{k k} \frac{\partial}{\partial u}$. Indeed, it is easy to see that

$$
\begin{aligned}
& \sum_{j \neq l}\left(\alpha_{j k} x_{j} \frac{\partial}{\partial u}+\beta_{j k} \frac{\partial}{\partial x_{j}}\right)\left(\alpha_{l k} \frac{\partial}{\partial x_{l}}-\beta_{l k} x_{l} \frac{\partial}{\partial u}\right) \\
= & \sum_{j \neq l}\left(\alpha_{l k} \frac{\partial}{\partial x_{l}}-\beta_{l k} x_{l} \frac{\partial}{\partial u}\right)\left(\alpha_{j k} x_{j} \frac{\partial}{\partial u}+\beta_{j k} \frac{\partial}{\partial x_{j}}\right) \\
= & \sum_{j \neq l}\left(\alpha_{j k} \frac{\partial}{\partial x_{j}}-\beta_{j k} x_{j} \frac{\partial}{\partial u}\right)\left(\alpha_{l k} x_{l} \frac{\partial}{\partial u}+\beta_{l k} \frac{\partial}{\partial x_{l}}\right) .
\end{aligned}
$$

Hence the imaginary part reduces to

$$
\begin{aligned}
& \sum_{j}\left(\alpha_{j k} x_{j} \frac{\partial}{\partial u}+\beta_{j k} \frac{\partial}{\partial x_{j}}\right)\left(\alpha_{j k} \frac{\partial}{\partial x_{j}}-\beta_{j k} x_{j} \frac{\partial}{\partial u}\right) \\
& -\sum_{j}\left(\alpha_{j k} \frac{\partial}{\partial x_{j}}-\beta_{j k} x_{j} \frac{\partial}{\partial u}\right)\left(\alpha_{j k} x_{j} \frac{\partial}{\partial u}+\beta_{j k} \frac{\partial}{\partial x_{j}}\right) \\
& =\sum_{j}\left(-\beta_{j k}^{2}-\alpha_{j k}^{2}\right) \frac{\partial}{\partial u}=-\sum_{j}\left|c_{j k}\right|^{2}=-a_{k k} \frac{\partial}{\partial u} .
\end{aligned}
$$


By using (4.3.9) together with the above calculations we obtain

$$
\mathcal{L}=-\sum_{k=1}^{n}\left[\sum_{j}\left(\alpha_{j k} \frac{\partial}{\partial x_{j}}-\beta_{j k} x_{j} \frac{\partial}{\partial u}\right)\right]^{2}+\left[\sum_{j}\left(\alpha_{j k} x_{j} \frac{\partial}{\partial u}+\beta_{j k} \frac{\partial}{\partial x_{j}}\right)\right]^{2} .
$$

For each $k=1, \cdots, n$ consider the real vector fields

$$
X_{k}:=\sum_{j}\left(\alpha_{j k} \frac{\partial}{\partial x_{j}}-\beta_{j k} x_{j} \frac{\partial}{\partial u}\right) \quad \text { and } \quad Y_{k}:=\sum_{j}\left(\alpha_{j k} x_{j} \frac{\partial}{\partial u}+\beta_{j k} \frac{\partial}{\partial x_{j}}\right) .
$$

It is clear that for each $k=1, \cdots, n$ we have $X_{k}^{\star}=-X_{k}$ and $Y_{k}^{\star}=-Y_{k}$ where $X_{k}^{\star}$ and $Y_{k}^{\star}$ denote the formal adjoint of $X_{k}$ and $Y_{k}$, respectively. This shows that the operator

$$
\mathcal{L}=-\sum_{k=1}^{n}\left(X_{k}^{2}+Y_{k}^{2}\right)=\sum_{k=1}^{n}\left(X_{k} X_{k}^{\star}+Y_{k} Y_{k}^{\star}\right)
$$

is symmetric and positive. Next, we show that for any fixed integer $p \in\{1, \cdots, n\}$ the linear combinations of elements in $\left\{X_{1}, \cdots, X_{n}, Y_{1}, \cdots, Y_{n},\left[X_{p}, Y_{p}\right]\right\}$ span the tangent space of $\mathbb{R}^{n+1}$ at each point. Fix a point $\left(x_{0}, u_{0}\right)=\left(x_{1}, \cdots, x_{n}, u_{0}\right) \in \mathbb{R}^{n}$ and an integer $p \in\{1, \cdots, n\}$. Denote by

$$
S=\operatorname{span}\left\{X_{1 \mid\left(x_{0}, u_{0}\right)}, \cdots, X_{n \mid\left(x_{0}, u_{0}\right)}, Y_{1 \mid\left(x_{0}, u_{0}\right)}, \cdots, Y_{n \mid\left(x_{0}, u_{0}\right)},\left[X_{p}, Y_{p}\right]_{\mid\left(x_{0}, u_{0}\right)}\right\}
$$

the linear span of the above vector fields at the point $\left(x_{0}, u_{0}\right)$. It follows that

$$
\begin{aligned}
{\left[X_{p}, Y_{p}\right]_{\mid\left(x_{0}, u_{0}\right)} } & =\sum_{j, l}\left[\alpha_{j p} \frac{\partial}{\partial x_{j}}-\beta_{j p} x_{j} \frac{\partial}{\partial u}, \alpha_{l p} x_{l} \frac{\partial}{\partial u}+\beta_{l p} \frac{\partial}{\partial x_{l}}\right]_{\mid\left(x_{0}, u_{0}\right)} \\
& =\sum_{j}\left[\alpha_{j p} \frac{\partial}{\partial x_{j}}-\beta_{j p} x_{j} \frac{\partial}{\partial u}, \alpha_{j p} x_{j} \frac{\partial}{\partial u}+\beta_{j p} \frac{\partial}{\partial x_{j}}\right]_{\mid\left(x_{0}, u_{0}\right)} \\
& =\left.\sum_{j}\left(\alpha_{j p}^{2}+\beta_{j p}^{2}\right) \frac{\partial}{\partial u}\right|_{\mid u_{0}}=\left.\sum_{j}\left|c_{j p}\right|^{2} \frac{\partial}{\partial u}\right|_{u_{0}}=\left.a_{p p} \frac{\partial}{\partial u}\right|_{\mid u_{0}} .
\end{aligned}
$$

Since $A+\bar{A}$ is positive definite this shows that $\left.\frac{\partial}{\partial u}\right|_{u_{0}} \in S$ and thus for any $k=1, \cdots, n$ the vector fields

$$
X_{k}^{\prime}:=X_{k \mid\left(x_{0}, u_{0}\right)}+\sum_{j} \beta_{j k} x_{j} \frac{\partial}{\partial u \mid u_{0}}=\sum_{j} \alpha_{j k} \frac{\partial}{\partial x_{j} x_{0}}
$$

and

$$
Y_{k}^{\prime}:=Y_{k \mid\left(x_{0}, u_{0}\right)}-\sum_{j} \alpha_{j k} x_{j} \frac{\partial}{\partial u}{ }_{\mid u_{0}}=\sum_{j} \beta_{j k} \frac{\partial}{\partial x_{j}}
$$

are elements of $S$. It is sufficient to prove that the vector fields $\left\{X_{1}^{\prime}, \cdots, X_{n}^{\prime}, Y_{1}^{\prime}, \cdots, Y_{n}^{\prime}\right\}$ span the tangent space of $\mathbb{R}^{n}$ at $x_{0}$. It is clear that the matrix of $\left\{X_{1}^{\prime}, \cdots, X_{n}^{\prime}\right\}$ (respectively of $\left.\left\{Y_{1}^{\prime}, \cdots, Y_{n}^{\prime}\right\}\right)$ in the canonical basis $\left\{\frac{\partial}{\partial x_{1} \mid x_{0}}, \cdots, \frac{\partial}{\partial x_{n} \mid x_{0}}\right\}$ is $\alpha$ (respectively $\beta$ ). Note that the real part of the matrix $A$ denoted by $\operatorname{Re}(A)$ and given by $\operatorname{Re}(A)=\frac{1}{2}(A+\bar{A})=\alpha^{2}-\beta^{2}$ is positive definite hence it is nonsingular. Therefore, for any $x \in \mathbb{R}^{n}$ there exists $v, w \in \mathbb{R}^{n}$ such 
that $v \in \operatorname{Im} \alpha$ and $w \in \operatorname{Im} \beta$ with $x=v+w$. This shows that $\operatorname{Im} \alpha+\operatorname{Im} \beta=\mathbb{R}^{n}$ which means that there are $n$ linearly independent column vectors formed by the matrices $\alpha$ and $\beta$. It follows that there are $n$ linearly independent vectors in $\left\{X_{1}^{\prime}, \cdots, X_{n}^{\prime}, Y_{1}^{\prime}, \cdots, Y_{n}^{\prime}\right\}$ ensuring the sub-ellipticity of $\mathcal{L}$

Remark 4.3.1. With the notation $(x, u, \eta, \theta)=\left(x_{1}, \cdots, x_{n}, u, \eta_{1}, \cdots, \eta_{n}, \theta\right) \in \mathbb{R}^{n+1} \times \mathbb{R}^{n+1}$ we write $p(x, u, \eta, \theta)$ for the principle symbol of the operator $\mathcal{L}$ given by (4.3.7). Using (4.3.8) it is easy to see that $p(x, u, \eta, \theta)$ is given by the matrix product

$$
\begin{aligned}
p(x, u, \eta, \theta) & =-\left(\left(\eta_{1}+i x_{1} \theta\right), \cdots,\left(\eta_{n}+i x_{n} \theta\right)\right) A\left(\left(\eta_{1}-i x_{1} \theta\right), \cdots,\left(\eta_{n}-i x_{n} \theta\right)\right)^{T} \\
& =-X^{T} A \bar{X}
\end{aligned}
$$

Recall that the characteristic set of $\mathcal{L}$ is defined to be the subset of $\mathbb{R}^{n+1} \times \mathbb{R}^{n+1} \backslash 0$ where the principle symbol $p(x, u, \eta, \theta)$ vanishes. Assume that $p(x, u, \eta, \theta)=0=X^{T} A \bar{X}$ with $X \in \mathbb{C}^{n}$. Since $A$ is positive semidefinite there exists a positive semidefinite matrix $C$ such that $A=C^{2}$ hence

$$
p(x, u, \eta, \theta)=X^{T} A \bar{X}=\left(C^{T} X\right)^{T} \overline{\left(C^{T} X\right)}=\left\|C^{T} X\right\|^{2}=0 \Longleftrightarrow X \in \operatorname{ker} C^{T}
$$

Therefore the characteristic set of $\mathcal{L}$ is the kernel of the matrix $C^{T}$. In particular, the linear space

$$
\{(0, \cdots, 0, u, 0, \cdots, 0, \theta) \mid u, \theta \in \mathbb{R}\}
$$

is in the characteristic set of $\mathcal{L}$. This shows that the operator $\mathcal{L}$ is not elliptic in $u$. Moreover, $\mathcal{L}$ is said to be of principle type if the gradient of the principle symbol (w.r.t. to $(\eta, \theta)$ ) does not vanish on the characteristic set of $\mathcal{L}$ (c.f. Section 1 in [73] and Section 2.3 in [147]). These types of differential operators are important for the local solvability of differential equations (see for example [108]). In our case the operator $\mathcal{L}$ is also not of principle type. Indeed, direct computation shows that for each $l=1, \cdots, n$ we have

$$
\frac{\partial p(x, u, \eta, \theta)}{\partial \eta_{l}}=-2 a_{l l} \eta_{l}+\sum_{k \neq l}\left[a_{l k}\left(-\eta_{k}+i x_{k} \theta\right)-a_{k l}\left(-\eta_{k}-i x_{k} \theta\right)\right]
$$

and

$$
\frac{\partial p(x, u, \eta, \theta)}{\partial \theta}=-2 \theta(x A x)
$$

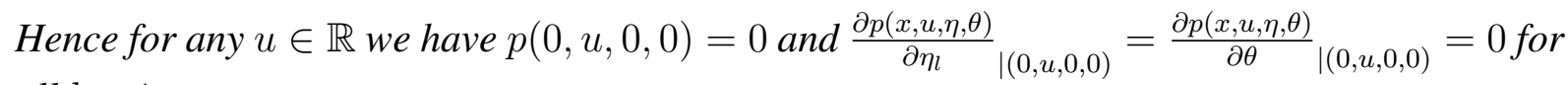
all $l=1, \cdots, n$.

From now on $\mathcal{L}$ will denote the operator defined in the above theorem satisfying the conditions there.

Since $\mathcal{L}$ is of the form (4.3.1) let us conjugate $\mathcal{L}$ w.r.t. the partial Fourier transform in the $u$-variable in order to eliminate the variable $u$. By (4.3.2) it follows that for every $\xi \in \mathbb{R}$ the 
operator $\mathcal{L}_{\xi}:=\mathcal{F}_{u} \mathcal{L F}_{u}^{-1}{ }_{(\cdot, \xi)}^{-1}$ is given by:

$$
\begin{aligned}
\mathcal{L}_{\xi}= & -\sum a_{j j} \frac{\partial^{2}}{\partial x_{j}^{2}}-2 \operatorname{Re} \sum_{j>k} a_{j k} \frac{\partial^{2}}{\partial x_{k} \partial x_{j}}-2 i \xi \operatorname{Im} \sum_{j>k} a_{j k}\left(x_{k} \frac{\partial}{\partial x_{j}}-x_{j} \frac{\partial}{\partial x_{k}}\right) \\
& +\xi^{2}\left(\sum a_{j j} x_{j}^{2}+2 \operatorname{Re} \sum_{j>k} a_{j k} x_{j} x_{k}\right) \\
= & \sum a_{j j}\left(\xi^{2} x_{j}^{2}-\frac{\partial^{2}}{\partial x_{j}^{2}}\right)+\sum_{j \neq k} a_{j k}\left(\xi x_{k}+\frac{\partial}{\partial x_{k}}\right)\left(\xi x_{j}-\frac{\partial}{\partial x_{j}}\right) .
\end{aligned}
$$

Let $\xi \neq 0$ be fixed and put $t=\sqrt{2|\xi|}$. Since $\mathcal{L}_{\xi}$ is a partial differential operator with polynomial coefficients on $L^{2}\left(\mathbb{R}^{n}\right)$ it follows that via the Bargmann transform $\beta_{t} \mathcal{L}_{\xi} \beta_{t}^{-1}$ is a Toeplitz operator with polynomial symbol acting on $H^{2}\left(\mathbb{C}^{n}\right)$. In order to calculate the exact symbol of the corresponding Toeplitz operator we need the following composition formula for the product of Toeplitz operators with polynomial symbols [63] (see also [14]).

Theorem 4.3.2. [63] Let $f$ an $g$ be two polynomials on $\mathbb{C}^{n}$. Then the operator product $T_{f} T_{g}$ is well defined on the dense domain

$$
\operatorname{span}\left\{p(z) e^{z a} \mid a \in \mathbb{C}^{n} \text { and } p \text { is a holomorphic polynomial on } \mathbb{C}^{n}\right\}
$$

Moreover, on this domain the following composition formula holds

$$
T_{f} T_{g}=T_{f \sharp g},
$$

where $f \sharp g$ is the polynomial given by

$$
f \sharp g(z)=\sum_{\gamma \in \mathbb{N}_{0}^{n}} \frac{(-1)^{|\gamma|}}{\gamma !}\left(\frac{\partial^{|\gamma|}}{\partial z^{\gamma}} f\right)(z)\left(\frac{\partial^{|\gamma|}}{\partial \bar{z}^{\gamma}} g\right)(z) .
$$

Using the above theorem together with Equations (4.2.10) and (4.2.11) we obtain the corresponding Toeplitz operator of $\mathcal{L}_{\xi}$ via the Bargmann transform $\beta \sqrt{2|\xi|}$.

Proposition 4.3.2. Let $\xi \neq 0$ be fixed and put $t=\sqrt{2|\xi|}$ then $\beta_{t} \mathcal{L}_{\xi} \beta_{t}^{-1}$ is the Toeplitz operator on $H^{2}\left(\mathbb{C}^{n}\right)$ given by

$$
\beta_{t} \mathcal{L}_{\xi} \beta_{t}^{-1}=2|\xi| T_{z A \bar{z}}-|\xi| \operatorname{tr}(A) .
$$

Proof. By Equations (4.2.10), (4.2.11) and (4.3.11) we obtain

1. For each $j=1, \cdots, n$ the following correspondence holds

$$
\begin{aligned}
\left(\xi^{2} x_{j}^{2}-\frac{\partial^{2}}{\partial x_{j}^{2}}\right) \longleftrightarrow & \frac{\xi^{2}}{t^{2}} T_{z_{j}+\bar{z}_{j}} T_{z_{j}+\bar{z}_{j}}-\frac{t^{2}}{4} T_{z_{j}-\bar{z}_{j}} T_{z_{j}-\bar{z}_{j}} \\
& =\frac{|\xi|}{2}\left[T_{\left(z_{j}+\bar{z}_{j}\right) \sharp\left(z_{j}+\bar{z}_{j}\right)}-T_{\left(z_{j}-\bar{z}_{j}\right) \sharp\left(z_{j}-\bar{z}_{j}\right)}\right] \\
& =\frac{|\xi|}{2}\left[T_{\left(z_{j}+\bar{z}_{j}\right)^{2}-1}-T_{\left(z_{j}-\bar{z}_{j}\right)^{2}+1}\right]=2|\xi| T_{\left|z_{j}\right|^{2}-\frac{1}{2}}
\end{aligned}
$$


2. For each $j \neq k$ we have

$$
\begin{gathered}
\left(\xi x_{k}+\frac{\partial}{\partial x_{k}}\right)\left(\xi x_{j}-\frac{\partial}{\partial x_{j}}\right) \longleftrightarrow \frac{|\xi|}{2}\left[T_{z_{k}+\bar{z}_{k}}+T_{z_{k}-\bar{z}_{k}}\right]\left[T_{z_{j}+\bar{z}_{j}}-T_{z_{j}-\bar{z}_{j}}\right] \\
=2|\xi| T_{z_{k} \sharp \bar{z}_{j}}=2|\xi| T_{z_{k} \bar{z}_{j}} .
\end{gathered}
$$

Substituting (4.3.14) and (4.3.15) into (4.3.10) we get

$$
\beta_{t} \mathcal{L}_{\xi} \beta_{t}^{-1}=2|\xi|\left[\sum a_{j j} T_{\left|z_{j}\right|^{2}-\frac{1}{2}}+\sum_{j \neq k} a_{j k} T_{z_{k} \bar{z}_{j}}\right]=2|\xi|\left[T_{z A \bar{z}}-\frac{1}{2} \operatorname{tr}(A)\right] .
$$

Let us now introduce the notion of the heat kernel of a Toeplitz operator.

Definition 4.3.3. For a measurable function $\Phi$ on $\mathbb{C}^{n}$ denote by $D\left(T_{\Phi}\right) \subset H^{2}\left(\mathbb{C}^{n}\right)$ the maximal domain of the Toeplitz operator $T_{\Phi}$. Assume that there is a function $K(s, z, w)$ defined on $\mathbb{R}_{+} \times \mathbb{C}^{n} \times \mathbb{C}^{n}$ such that $K(0, z, w)=e^{z \bar{w}}$ and $K(s, \cdot, w) \in D\left(T_{\Phi}\right)$ for all $s \geq 0, w \in \mathbb{C}^{n}$. Moreover, suppose that

$$
\left(\frac{\partial}{\partial s}+T_{\Phi}\right) K(s, \cdot, w)=0 \quad \text { for all } \quad s \geq 0, w \in \mathbb{C}^{n} .
$$

Then the function $K$ is called the heat kernel of the Toeplitz operator $T_{\Phi}$.

In the following, we aim to calculate the ,heat kernel” of the essentially selfadjoint semibounded operator $\beta_{t} \mathcal{L}_{\xi} \beta_{t}^{-1}$ given by (4.3.13) on the Segal-Bargmann space $H^{2}\left(\mathbb{C}^{n}\right)$. The main point is obviously to calculate the ,heat kernel" of the Toeplitz operator $T_{z A \bar{z}}$ on $H^{2}\left(\mathbb{C}^{n}\right)$. For this reason, we introduce Berezin's result on the exponential of an essentially selfadjoint Toeplitz operators with positive symbols.

Theorem 4.3.3. [29] Let $f$ be a positive function a.e. on $\mathbb{C}^{n}$ and suppose that the Toeplitz operator $T_{f}$ is essentially selfadjoint on a dense subset of $H^{2}\left(\mathbb{C}^{n}\right)$ then for any $s>0$ we have:

$$
e^{-s T_{f}}=\lim _{N \longrightarrow \infty}\left(T_{e^{-\frac{s}{N} f}}\right)^{N},
$$

where the limit is in the strong sense.

The above theorem expresses the kernel of the exponential of the Toeplitz operator $\left(-s T_{f}\right)$ as the limit of a multiple integral over $\mathbb{C}^{n N}$ obtained by the higher product of Toeplitz operators $\left(T_{e^{-\frac{s}{N}} f}\right)^{N}$. However, in some cases this multiple integral can be reduced to an integral over $\mathbb{C}^{n}$. For example, this holds true if the higher products $\left(T_{e^{-\frac{s}{N}} f}\right)^{N}$ are also Toeplitz operators. Now let us apply the above theorem to the case $f(z)=z A \bar{z}$.

In order to show that the Toeplitz operator $T_{z A \bar{z}}$ is essentially selfadjoint we recall the notion of a $t$-radially symmetric function which was first introduced by E. Fischer in [82].

Let $t=\left(t_{1}, \cdots, t_{n}\right) \in \mathbb{R}_{+}^{n}$. A measurable function $\varphi$ on $\mathbb{C}^{n}$ is said to be $t$-radially symmetric if for each $\theta \in \mathbb{R}$ we have

$$
\varphi\left(e^{i t_{1} \theta} z_{1}, \cdots, e^{i t_{n} \theta} z_{n}\right)=\varphi(z), \quad \text { a.e. } z=\left(z_{1}, \cdots, z_{n}\right) \in \mathbb{C}^{n} .
$$


The next theorem shows the essentially selfadjointness of Toeplitz operators with $t$-radially symmetric real valued symbols. This theorem is a consequence of Theorem 4.1 and Proposition 5.2 in [118].

Theorem 4.3.4. [118] Let $\varphi: \mathbb{C}^{n} \longrightarrow \mathbb{R}$ be a t-radially symmetric function and suppose that the space of the holomorphic polynomials $\mathbb{P}[z]$ is contained in $D\left(T_{\varphi}\right)$. Denote by $\left.T_{\varphi}\right|_{\mathbb{P}}$ the restriction of the Toeplitz operator $T_{\varphi}$ to $\mathbb{P}[z]$. Then the closure $\left(\left.T_{\varphi}\right|_{\mathbb{P}}\right)^{-}$is selfadjoint and consequently $T_{\varphi}$ is essentially selfadjoint as an operator on $\mathbb{P}[z] \subset H^{2}\left(\mathbb{C}^{n}\right)$.

Note that the matrix $A$ is positive semidefinite i.e. $z A \bar{z} \geq 0$. Moreover, it is obvious that the function $f(z):=z A \bar{z}$ satisfies $f\left(e^{i \theta} z\right)=f(z)$ for all $z \in \mathbb{C}^{n}$ showing that $f$ is $(1, \cdots, 1)$ radially symmetric. Therefore, by the above theorem we know that $T_{z A \bar{z}}$ is essentially selfadjoint as an operator on $\mathbb{P}[z] \subset H^{2}\left(\mathbb{C}^{n}\right)$. This shows that Equation (4.3.16) holds true for $f(z):=z A \bar{z}$. Moreover, by calculating the higher products $\left(T_{e^{-\frac{s}{N}} f}\right)^{N}$ for all $N \in \mathbb{N}$ we prove that the operator $e^{-s T_{z A \bar{z}}}$ is also a Toeplitz operator on $H^{2}\left(\mathbb{C}^{n}\right)$. In the next theorem, we obtain a composition formula $\left(T_{e^{z B \bar{z}}}\right)^{N}$ whenever $I d-B$ is a positive definite Hermitian matrix. We then apply the result to the case $B=-\frac{s}{N} A$ proving that $\left(T_{e^{-\frac{s}{N} f}}\right)^{N}$ is a Toeplitz operator on $H^{2}\left(\mathbb{C}^{n}\right)$.

Proposition 4.3.3. Let $B \in M_{n}(\mathbb{C})$ be a $n \times n$ matrix such that $I d-B$ is a positive definite Hermitian matrix. Then for any $N \in \mathbb{N}$ the product $\left(T_{e^{z B \bar{z}}}\right)^{N}$ is well defined on the dense domain

$$
\mathcal{D}:=\operatorname{span}\left\{p(z) e^{z a} \mid a \in \mathbb{C}^{n} \text { and } p \text { is a holomorphic polynomial on } \mathbb{C}^{n}\right\} \subset H^{2}\left(\mathbb{C}^{n}\right)
$$

and is given by

$$
\left(T_{e^{z B \bar{z}}}\right)^{N}=T_{e^{z\left\{-(I d-B)^{N}+I d\right\} \bar{z}}} .
$$

Proof. Let us first check that $T_{e^{z B z}}$ is well defined on $\mathcal{D}$. For any $g \in \mathcal{D}$ we have

$$
\begin{aligned}
\int_{\mathbb{C}^{n}}|g(w)|^{2} e^{2 w B \bar{w}} e^{-|w|^{2}} d v(w) & =\int_{\mathbb{C}^{n}}|g(w)|^{2} e^{-\frac{w}{2}(2 I d-B) \bar{w}} d v(w) \\
& =\operatorname{det}(2 I d-B)^{-1} \int_{\mathbb{C}^{n}}|g(\sqrt{(2 I d-\bar{B})-1} u)|^{2} e^{-\frac{|u|^{2}}{2}} d v(u)<\infty
\end{aligned}
$$

where in the last equality we used the change of variable $u=\sqrt{(2 I d-\bar{B})} w$ which is well defined since $2 I d-\bar{B}$ is positive definite. Now fix $z \in \mathbb{C}^{n}$ and let us calculate $T_{e^{z B \bar{z}}} g(z)$. Using the change of variable $u=\sqrt{(I d-\bar{B})} w$ we have

$$
\begin{aligned}
T_{e^{z B \bar{z}}} g(z) & =\pi^{-n} \int g(w) e^{w B \bar{w}} e^{z \bar{w}} e^{-|w|^{2}} d v(w) \\
& =\pi^{-n} \int g(w) e^{-w(I d-B) \bar{w}} e^{z \bar{w}} d v(w) \\
& =\pi^{-n} \int g\left(\sqrt{(I d-\bar{B})^{-1}} u\right) e^{z \sqrt{(I d-B)^{-1}} \bar{u}} e^{-|u|^{2}} d v\left(\sqrt{(I d-\bar{B})^{-1}} u\right) \\
& =\operatorname{det}(I d-B)^{-1} g\left((I d-\bar{B})^{-1} z\right) .
\end{aligned}
$$


This shows that $\mathcal{D}$ is invariant under $T_{e^{z B \bar{z}}}$ and thus $\left(T_{e^{z B \bar{z}}}\right)^{N}$ is well defined on $\mathcal{D}$ for all $N \in \mathbb{N}$. Moreover, using (4.3.18) we prove by induction Eq. (4.3.17). Fix $N \in \mathbb{N}$ and suppose that (4.3.17) holds true for all integers less than or equal $N-1$. Then for any $g \in \mathcal{D}$ we have

$$
\begin{aligned}
{\left[\left(T_{e^{z B \bar{z}}}\right)^{N} g\right](z) } & =T_{e^{z B \bar{z}}}\left[T_{e^{z\left\{-(I d-B)^{N-1}+I d\right\} \bar{z}}} g\right](z) \\
& =\left(\operatorname{det}(I d-B)^{-1}\right)^{N-1}\left[T_{e^{z B \bar{z}}} g\left(\left\{I d+(I d-\bar{B})^{N-1}-I d\right\}^{-1}(\cdot)\right)\right](z) \\
& =\left(\operatorname{det}(I d-B)^{-1}\right)^{N} g\left(\left[(I d-\bar{B})^{N}\right]^{-1} z\right) \\
& =T_{e^{z\left\{-(I d-B)^{N}+I d\right\} \bar{z}}} g(z) .
\end{aligned}
$$

As a consequence we prove that for every $s>0$ the operator $e^{-s T_{z A \bar{z}}}$ is a Toeplitz operator densely defined on $\mathcal{D} \subset H^{2}\left(\mathbb{C}^{n}\right)$. Indeed, since $A$ is positive semidefinite then $I d+\frac{s}{N} A$ is a positive definite matrix for all $s>0$ and all $N \in \mathbb{N}$. Therefore, applying Proposition 4.3.3 for $B=-\frac{s}{N} A$ we obtain

$$
\begin{aligned}
e^{-s T_{z A \bar{z}}} & =\lim _{N \longrightarrow \infty}\left(T_{\left.e^{-z \frac{s}{N} A \bar{z}}\right)^{N}}\right. \\
& =\lim _{N \longrightarrow \infty} T_{e^{z\left[-\left(I d+\frac{s}{N} A\right)^{N}+I d\right] \bar{z}}} \\
& =T_{e^{z\left(-e^{s A}+I d\right) \bar{z}}}
\end{aligned}
$$

where the limit is in the strong sense. In fact, the above equation holds true by applying the Lebesgue dominated convergence theorem twice. More precisely, denote by $\left\{g_{N}\right\}_{N \in \mathbb{N}}$ the sequence of functions in $L^{2}\left(\mathbb{C}^{n}, d \mu\right)$ defined by

$$
g_{N}(w):=e^{w\left[-\left(I d+\frac{s}{N} A\right)^{N}+I d\right] \bar{w}} .
$$

Then $g_{N}$ converges pointwisely to $g(w):=e^{w\left(-e^{s A}+I d\right) \bar{w}} \in L^{2}\left(\mathbb{C}^{n}, d \mu\right)$. Moreover, since $A$ is positive semidefinite it follows that $g_{N} \leq g_{1}$ for all $N \in \mathbb{N}$. Hence, for every $f \in \mathcal{D}$ the sequence of functions $T_{g_{N}} f$ converges to $T_{g} f$ pointwisely on $\mathbb{C}^{n}$. Furthermore, for each $N \in \mathbb{N}$ it is easy to check that

$$
\left|T_{g_{N}} f(z)\right|=\left|\int_{\mathbb{C}^{n}} f(w) g_{N}(w) K_{\bar{z}}(w) d \mu(w)\right| \leq \int_{\mathbb{C}^{n}}\left|f(w) g_{1}(w) K_{\bar{z}}(w)\right| d \mu(w) \in L^{2}\left(\mathbb{C}_{z}^{n}, d \mu\right) .
$$

Which shows that $T_{g_{N}} f \stackrel{N \rightarrow \infty}{\longrightarrow} T_{g} f$ in $H^{2}\left(\mathbb{C}^{n}\right)$ for each $f \in \mathcal{D}$, i.e. $T_{g_{N}}$ converges to $T_{g}$ in the strong sense.

We are able now to obtain the ,heat kernel” of $T_{z A \bar{z}}$ on $H^{2}\left(\mathbb{C}^{n}\right)$.

Theorem 4.3.5. Let $A \in M_{n}(\mathbb{C})$ be a $n \times n$ positive semidefinite matrix. Then the heat kernel of the Toeplitz operator $T_{z A \bar{z}}$ defined on $\mathcal{D} \subset H^{2}\left(\mathbb{C}^{n}\right)$ is given by

$$
K_{A}(s, z, w)=e^{-s \operatorname{tr}(A)} e^{z e^{-s A} \bar{w}}, \quad(s, z, w) \in \mathbb{R}_{+} \times \mathbb{C}^{n} \times \mathbb{C}^{n} .
$$


Proof. Fix $s>0$ and let $B=-e^{s A}+I d$. Then by Equations (4.3.18) and (4.3.19) together with the reproducing kernel property of the Segal-Bargmann space we have

$$
\left\langle e^{-s T_{z A \bar{z}}} K_{w}, K_{z}\right\rangle=\left\langle T_{e^{z B \bar{z}}} K_{w}, K_{z}\right\rangle=\operatorname{det}(I d-B)^{-1}\left\langle e^{\cdot(I d-B)^{-1} \bar{w}}, e^{\cdot \bar{z}}\right\rangle=e^{-s \operatorname{tr}(A)} e^{z e^{-s A} \bar{w}} .
$$

To see that the above equation is the heat kernel of $T_{z A \bar{z}}$ in $\mathcal{D} \subset H^{2}\left(\mathbb{C}^{2 n}\right)$. One can easily check that $K_{A}(0, z, w)=e^{z \cdot \bar{w}}$ and that

$$
\left(\frac{\partial}{\partial s}+T_{z A \bar{z}}\right)\left\langle e^{-s T_{z A \bar{z}}} K_{w}, K_{z}\right\rangle=0 \text { for all } w \in \mathbb{C}^{n} .
$$

In the next proposition for a fixed $s>0$ we investigate if the entire function $K_{A}(s, z, \bar{w})$ is in the range of the Bargmann transform when restricted to the Schwartz space. It turns out that such a property holds independent of the time variable $s$.

Theorem 4.3.6. Let $A$ be a positive semidefinite matrix and $K_{A}(s, z, w)$ be the heat kernel of the Toeplitz operator $T_{z A \bar{z}}$ obtained in the above theorem. Then there exists a unitary matrix $P$ such that

$$
K_{A}(s, z, w)=e^{-s \operatorname{tr}(A)} \sum_{\alpha \in \mathbb{N}_{0}^{n}} \frac{e^{-s \lambda \cdot \alpha}}{\alpha !}\left(P^{T} z\right)^{\alpha}\left(\overline{P^{-1} w}\right)^{\alpha},
$$

where $\lambda \cdot \alpha=\sum_{j=1}^{n} \lambda_{j} \alpha_{j}$ and each $\lambda_{j}$ is an eigenvalue of $A$ repeated according to its multiplicity. Moreover, for every $s, t>0$ the entire function $K_{A}(s, z, \bar{w}) \in \beta_{t}\left[\mathcal{S}\left(\mathbb{R}^{2 n}\right)\right]$ if and only if $A$ is positive definite.

Proof. Since $A$ is positive semidefinite there is a unitary matrix $P$ such that

$$
A=P D\left(\lambda_{1}, \cdots, \lambda_{n}\right) P^{-1}
$$

where $D\left(\lambda_{1}, \cdots, \lambda_{n}\right)$ is the diagonal matrix whose entries $\lambda_{j} \geq 0$ are the eigenvalues of $A$. Using the change of variables $z \longmapsto \bar{P} z$ and $w \longmapsto P w$ we can write

$$
\begin{aligned}
e^{s \operatorname{tr}(A)} K_{A}(s, \bar{P} z, \overline{P w}) & =e^{z P^{-1} P D\left(e^{-s \lambda_{1}}, \cdots, e^{-s \lambda_{n}}\right) P^{-1} P w}=e^{z D\left(e^{-s \lambda_{1}}, \cdots, e^{-s \lambda_{n}}\right) w} \\
& =\prod_{j=1}^{n} e^{e^{-s \lambda_{j}} z_{j} w_{j}}=\prod_{j=1}^{n} \sum_{\alpha_{j} \in \mathbb{N}_{0}} \frac{1}{\alpha_{j} !} e^{-s \lambda_{j} \alpha_{j}}\left(z_{j} w_{j}\right)^{\alpha_{j}} \\
& =\sum_{\alpha \in \mathbb{N}_{0}^{n}} \frac{e^{-s \lambda \cdot \alpha}}{\alpha !} z^{\alpha} \bar{w}^{\alpha}
\end{aligned}
$$

On the one hand, replacing $z$ by $P^{T} z$ and $w$ by $P^{-1} w$ in the above equation we obtain (4.3.21). On the other hand, since $z \otimes w \longmapsto \bar{P} z \otimes P w$ is a unitary operator on $\mathbb{C}^{n} \otimes \mathbb{C}^{n}$ it follows that for every $t>0$

$$
\beta_{t}^{-1}\left[K_{A}(s, z, \bar{w})\right] \in \mathcal{S}\left(\mathbb{R}^{2 n}\right) \Longleftrightarrow \beta_{t}^{-1}\left[\sum_{\alpha \in \mathbb{N}_{0}^{n}} \frac{e^{-s \lambda \cdot \alpha}}{\alpha !} z^{\alpha} \bar{w}^{\alpha}\right] \in \mathcal{S}\left(\mathbb{R}^{2 n}\right) .
$$


For every $j=1, \cdots, n$ denote by $\beta_{t, j}^{-1}$ the inverse Bargmann transform at $t>0$ on the SegalBargmann space over $\mathbb{C}^{2}$ w.r.t. the coordinates $\left(z_{j}, w_{j}\right) \in \mathbb{C}^{2}$ then

$$
\begin{aligned}
\beta_{t}^{-1}\left[\sum_{\alpha \in \mathbb{N}_{0}^{n}} \frac{e^{-s \lambda \cdot \alpha}}{\alpha !} z^{\alpha} \bar{w}^{\alpha}\right] & =\beta_{t}^{-1}\left[\prod_{j=1}^{n} \sum_{\alpha_{j} \in \mathbb{N}_{0}} \frac{1}{\alpha_{j} !} e^{-s \lambda_{j} \alpha_{j}}\left(z_{j} w_{j}\right)^{\alpha_{j}}\right] \\
& =\prod_{j=1}^{n} \beta_{t, j}^{-1}\left[\sum_{\alpha_{j} \in \mathbb{N}_{0}} \frac{1}{\alpha_{j} !} e^{-s \lambda_{j} \alpha_{j}}\left(z_{j} w_{j}\right)^{\alpha_{j}}\right] .
\end{aligned}
$$

This shows that $\beta_{t}^{-1}\left[K_{A}(s, z, \bar{w})\right] \in \mathcal{S}\left(\mathbb{R}^{2 n}\right)$ if and only if for every $j=1, \cdots, n$

$$
\sum_{\alpha_{j} \in \mathbb{N}_{0}} \frac{1}{\alpha_{j} !} e^{-s \lambda_{j} \alpha_{j}}\left(z_{j} w_{j}\right)^{\alpha_{j}} \in \beta_{t}\left[\mathcal{S}\left(\mathbb{R}^{2}\right)\right] .
$$

Using Proposition 4.2.1, this is equivalent to

$$
\sup _{\alpha \in \mathbb{N}_{0}}\left(e^{-s \lambda_{j} \alpha} \alpha^{2 M}\right)<\infty \quad \text { for all } \quad M>0 \Longleftrightarrow \lambda_{j}>0
$$

Remark 4.3.2. Similar to Proposition 4.2.1, there is a characterization for the image of the space of tempered distributions under the Bargmann transform (c.f. Proposition 2.6 of Chapter 4 in [139]). We note that under the assumption that $A$ is positive semidefinite one can show that $K_{A}(s, z, \bar{w}) \in \beta_{t}\left[\mathcal{S}^{\prime}\left(\mathbb{R}^{2 n}\right)\right]$ where $\mathcal{S}^{\prime}\left(\mathbb{R}^{2 n}\right)$ denotes the space of tempered distributions. In fact, this follows from the fact that $\sup \left(e^{-s \lambda_{j} \alpha} \alpha^{-2 M}\right)<\infty$ for all $M>0$.

Note that the above results are valid for any positive semidefinite matrix without the assumptions $(A+\bar{A})$ is positive definite or $\bar{A} A=A \bar{A}$. However, we will need these conditions in order to obtain the heat kernel of $\mathcal{L}_{\xi}$ (c.f. the proof of Corollary 4.3.2).

Remark 4.3.3. We show that the Toeplitz operator $T_{z A \bar{z}}$ is diagonalizable. Moreover, we prove that in the case where $A$ is positive definite $T_{z A \bar{z}}$ has a discrete spectrum and each eigenvalue has a finite multiplicity. Indeed, with the notation used in Theorem 4.3.6 define the unitary operator $U_{P}$ on $H^{2}\left(\mathbb{C}^{n}\right)$ by

$$
\left[U_{P} f\right](z):=f \circ P^{T}(z) .
$$

Therefore, for any $f \in D \subset H^{2}\left(\mathbb{C}^{n}\right)$ we can write

$$
\begin{aligned}
T_{z A \bar{z}} f(z) & =\int_{\mathbb{C}^{n}} w A \bar{w} f(w) e^{z \bar{w}} d \mu(w)=\int_{\mathbb{C}^{n}} w P D\left(\lambda_{1}, \cdots, \lambda_{n}\right) P^{-1} \bar{w} f(w) e^{z \bar{w}} d \mu(w) \\
& =\int_{\mathbb{C}^{n}}\left(P^{T} w\right)^{T} D\left(\lambda_{1}, \cdots, \lambda_{n}\right) \overline{\left(P^{T} w\right)} f(w) e^{z \bar{w}} d \mu(w) \\
& =\sum_{j=1}^{n} \lambda_{j} \int_{\mathbb{C}^{n}}\left|\left(P^{T} w\right)_{j}\right|^{2} f(w) e^{z \bar{w}} d \mu(w)=\sum_{j=1}^{n} \lambda_{j} \int_{\mathbb{C}^{n}}\left|u_{j}\right|^{2} f(\bar{P} u) e^{z P \bar{w}} d \mu(u) \\
& =\sum_{j=1}^{n} \lambda_{j}\left[T_{\left|z_{j}\right|^{2}}\left(U_{P}^{\star} f\right)\right]\left(P^{T} z\right)=\sum_{j=1}^{n} \lambda_{j} U_{P}\left[T_{\left|z_{j}\right|^{2}} U_{P}^{\star} f\right](z),
\end{aligned}
$$


where we used the change of variable $u=P^{T} w$. This shows that $T_{z A \bar{z}}$ can be written in the following form

$$
T_{z A \bar{z}}=\sum_{j=1}^{n} \lambda_{j} U_{P} T_{\left|z_{j}\right|^{2}} U_{P}^{\star}
$$

The last expression can be used to obtain the eigenfunctions and eigenvalues of $T_{z A \bar{z}}$. In fact, for each $j=1, \cdots, n$ and each $\alpha \in \mathbb{N}_{0}^{n}$ we have

$$
T_{\left|z_{j}\right|^{2}} z^{\alpha}=\left(z_{j} \frac{\partial}{\partial z_{j}}+I\right) z^{\alpha}=\left(\alpha_{j}+1\right) z^{\alpha} .
$$

This shows that the Toeplitz operator $T_{z A \bar{z}}$ has eigenfunctions $\left\{U_{P} z^{\alpha}=\left(P^{T} z\right)^{\alpha}\right\}_{\alpha \in \mathbb{N}_{0}^{n}}$ with the corresponding eigenvalues

$$
\lambda_{\alpha}=\sum_{j=1}^{n} \lambda_{j}\left(\alpha_{j}+1\right) .
$$

(Note that since $T_{z A \bar{z}}$ is a symmetric operator it follows by Lemma 1.2.2 in [68] that $T_{z A \bar{z}}$ is essentially selfadjoint). Hence in case where $A$ is positive definite it follows that $\lim _{|\alpha| \rightarrow \infty} \lambda_{\alpha}=$ $\infty$ and each eigenvalue has finite multiplicity showing that the spectrum of $T_{z A \bar{z}}$ is discrete (c.f. Theorem C.2.4 in [131]). Note that if $A$ is singular then the eigenvalues of $T_{z A \bar{z}}$ are of infinite multiplicity (Example 4.3.2).

Theorem 4.3.5 together with an easy computation gives the ,heat kernel” of the Toeplitz operator (4.3.13).

Corollary 4.3.1. Let $A \in M_{n}(\mathbb{C})$ be a $n \times n$ positive semidefinite matrix. Then for any $\xi \neq 0$ the ,heat kernel" of the Toeplitz operator $\left[2|\xi| T_{z A \bar{z}}-|\xi| \operatorname{tr}(A)\right]$ denoted by $K_{\xi}(s, z, w)$ is given by

$$
K_{\xi}(s, z, w)=e^{-s|\xi| \operatorname{tr}(A)} e^{z e^{-2 s|\xi| A} \bar{w}}, \quad(s, z, w) \in \mathbb{R}_{+} \times \mathbb{C}^{n} \times \mathbb{C}^{n}
$$

By the above corollary and using the inverse Bargmann transform we are able now to calculate the heat kernel of the partial differential operator $\mathcal{L}_{\xi}$ on $L^{2}\left(\mathbb{R}^{n}\right)$ whenever $A \bar{A}=\bar{A} A$ and the matrix $(A+\bar{A})$ is positive definite.

Corollary 4.3.2. Let $A=\left(a_{j k}\right) \in M_{n}(\mathbb{C})$ be an $n \times n$ positive semidefinite matrix. For each $\xi \neq 0$ consider the differential operator $\mathcal{L}_{\xi}$ on $\mathbb{R}^{n}$ defined by

$$
\mathcal{L}_{\xi}=\sum a_{j j}\left(\xi^{2} x_{j}^{2}-\frac{\partial^{2}}{\partial x_{j}^{2}}\right)+\sum_{j \neq k} a_{j k}\left(\xi x_{k}+\frac{\partial}{\partial x_{k}}\right)\left(\xi x_{j}-\frac{\partial}{\partial x_{j}}\right) .
$$

Then $\mathcal{L}_{\xi}$ is essentially selfadjoint as an operator on the following dense domain of $L^{2}\left(\mathbb{R}^{n}\right)$

$$
\mathcal{D}_{\xi}:=\left\{p(x) e^{-|\xi| \frac{x^{2}}{2}} \mid p \text { is a polynomial over } \mathbb{R}^{n}\right\} .
$$

Moreover, $\left\langle\mathcal{L}_{\xi} \varphi, \varphi\right\rangle_{L^{2}\left(\mathbb{R}^{n}\right)} \geq-|\xi|\|\varphi\|^{2}$ for all $\varphi \in \mathcal{D}_{\xi}$. Furthermore, if $A$ and $\bar{A}$ commute and $(A+\bar{A})$ is positive definite then the heat kernel of $\mathcal{L}_{\xi}$ denoted by $k_{\xi}(s, x, y)$ and defined on 
$\mathbb{R}_{+} \times \mathbb{R}^{n} \times \mathbb{R}^{n}$ is given by

$$
\begin{aligned}
k_{\xi}(s, x, y)= & e^{-s|\xi| \operatorname{tr}(A)}\left(\frac{|\xi|}{\pi}\right)^{\frac{n}{2}} \sqrt{\operatorname{det}\left(I d-e^{-2 s|\xi|(A+\bar{A})}\right)^{-1}} \\
& \times \exp \left\{-\frac{|\xi|}{2} y\left(I d-e^{-2 s|\xi|(A+\bar{A})}\right)^{-1}\left(I d+e^{-2 s|\xi|(A+\bar{A})}\right) y\right. \\
& -\frac{|\xi|}{2} x\left(I d-e^{-2 s|\xi|(A+\bar{A})}\right)^{-1}\left(I d+e^{-2 s|\xi|(A+\bar{A})}\right) x \\
& \left.+2|\xi| y\left(I d-e^{-2 s|\xi|(A+\bar{A})}\right)^{-1} e^{-2 s|\xi| A} x\right\} .
\end{aligned}
$$

Proof. Let $\xi \neq 0, s>0$ be fixed and put $t=\sqrt{2|\xi|}$. Since $T_{z A \bar{z}}$ is essentially selfadjoint as an operator on $\mathbb{P}[z]$ the differential operator $\mathcal{L}_{\xi}$ is essentially selfadjoint on the space of functions

$$
\mathcal{D}_{\xi}^{\prime}:=\operatorname{span}\left\{h_{\alpha}:=\beta_{t}^{-1} z^{\alpha}, \alpha \in \mathbb{N}_{0}^{n}\right\}
$$

Since the space of holomorphic polynomials is dense in $H^{2}\left(\mathbb{C}^{n}\right)$ it follows that $\mathcal{D}_{\xi}^{\prime}$ is dense in $L^{2}\left(\mathbb{R}^{n}\right)$. However, one can easily check that $\mathcal{D}_{\xi}=\mathcal{D}_{\xi}^{\prime}$. Indeed, using Equation (4.2.1) we know that $h_{0}(x)=t^{\frac{n}{2}}(2 \pi)^{-\frac{n}{4}} e^{-t^{2} \frac{x^{2}}{4}}$. Hence, by (4.2.12) we obtain

$$
\begin{aligned}
h_{\alpha}(x)=\beta_{t}^{-1} z^{\alpha} & =\beta_{t}^{-1}\left[T_{z^{\alpha}} 1\right](x)=\beta_{t}^{-1} T_{z^{\alpha}} \beta_{t} h_{0}(x) \\
& =t^{\frac{n}{2}}(2 \pi)^{-\frac{n}{4}}\left(\frac{t}{2} x_{1}-\frac{1}{t} \frac{\partial}{\partial x_{1}}\right)^{\alpha_{1}} \cdots\left(\frac{t}{2} x_{n}-\frac{1}{t} \frac{\partial}{\partial x_{n}}\right)^{\alpha_{n}} e^{-t^{2} \frac{x^{2}}{4}} \in \mathcal{D}_{\xi} .
\end{aligned}
$$

The other inclusion $\mathcal{D}_{\xi} \subset \mathcal{D}_{\xi}^{\prime}$ holds true by a similar argument. We conclude that $\mathcal{L}_{\xi}$ is essentially selfadjoint on $\mathcal{D}_{\xi}$. Moreover, since $A$ is positive semidefinite the Toeplitz operator $T_{z A \bar{z}}$ is non-negative on its maximal domain $D\left(T_{z A \bar{z}}\right)$. Consequently, and via the Bargmann transform it follows that $\mathcal{L}_{\xi}+|\xi| \operatorname{tr}(A)$ is a non-negative operator on $\left\{\beta_{t}^{-1}(g), g \in D\left(T_{z A \bar{z}}\right)\right\} \supset \mathcal{D}_{\xi}$.

Let us now calculate the heat kernel of $\mathcal{L}_{\xi}$. By Corollary 4.3.1 the heat kernel $K_{\xi}(s, z, w)$ of the operator $\beta_{t} \mathcal{L}_{\xi} \beta_{t}^{-1}=2|\xi| T_{z A \bar{z}}-|\xi| \operatorname{tr}(A)$ on $H^{2}\left(\mathbb{C}^{n}\right)$ is given by (4.3.22) where $(s, z, w) \in$ $\mathbb{R}_{+} \times \mathbb{C}^{n} \times \mathbb{C}^{n}$. However, Example 4.2.1 shows that the heat kernel of the operator $\mathcal{L}_{\xi}$ on $L^{2}\left(\mathbb{R}^{n}\right)$ denoted by $k_{\xi}(s, x, y)$ is given by $k_{\xi}(s, x, y)=\left[\beta_{t, y \rightarrow w}^{-1}\left[\beta_{t, x \rightarrow z}^{-1} K_{\xi}(s, z, \bar{w})\right](x)\right](y)=e^{-s|\xi| \operatorname{tr}(A)}\left[\beta_{t, y \rightarrow w}^{-1}\left[\beta_{t, x \rightarrow z}^{-1} e^{z e^{-2 s|\xi|} w}\right](x)\right](y)$,

where $(s, z, w) \in \mathbb{R}_{+} \times \mathbb{C}^{n} \times \mathbb{C}^{n}$ and $t=\sqrt{2|\xi|}$. Direct application of Proposition 4.2.4 to the matrix $e^{-2 s|\xi| A}$ gives the exact expression of (4.3.25) and shows that $k_{\xi}(s, x, y)$ is given by (4.3.24).

Remark 4.3.4. Using (4.3.23) one can easily check that the principle symbol $p(x, \eta)$ of the operator $\mathcal{L}_{\xi}$ is given by

$$
p(x, \eta)=-\frac{1}{2} \eta(A+\bar{A}) \eta, \quad(x, \eta) \in \mathbb{R}^{n} \times \mathbb{R}^{n} .
$$

So that in the case where $(A+\bar{A})$ is positive definite the operator $\mathcal{L}_{\xi}$ is elliptic. 
Remark 4.3.5. It is easy to see that the heat kernel (4.3.24) satisfies $k_{\xi}(s, x, y)=k_{-\xi}(s, x, y)$. Moreover, the function $k(s, x, y, \xi):=k_{\xi}(s, x, y) \in C^{\infty}\left((0, \infty) \times \mathbb{R}^{2 n} \times \mathbb{R}\right)$. Indeed, fix $s>0$ and $x, y \in \mathbb{R}^{2 n}$ where we write

$$
k(s, x, y, \xi)=f(|\xi|) e^{-g(x, y,|\xi|)},
$$

and $f$ is the function defined $(0, \infty)$ by

$$
f(v):=\frac{1}{\pi^{\frac{n}{2}}} e^{-s v \operatorname{tr}(A)} v^{\frac{n}{2}} \sqrt{\operatorname{det}\left(I d-e^{-2 s v(A+\bar{A})}\right)^{-1}} .
$$

Moreover, $e^{-g(x, y,|\xi|)}$ is the exponential part in (4.3.24). The function $f$ can be extended to the real line $\mathbb{R}$ and is a Schwartz function. In fact, since the matrix $(A+\bar{A})$ is positive definite and real then it is diagonalizable by a real unitary matrix $Q$ i.e.

$$
A+\bar{A}=Q^{-1} D\left(\lambda_{1}, \cdots, \lambda_{n}\right) Q
$$

where $\lambda_{1}, \cdots, \lambda_{n}$ are the eigenvalues of the matrix $A+\bar{A}$. This shows that

$$
\left(I d-e^{-2 s v(A+\bar{A}}\right)^{-1}=Q D\left(\frac{1}{1-e^{-2 s v \lambda_{1}}}, \cdots, \frac{1}{1-e^{-2 s v \lambda_{n}}}\right) Q^{-1} .
$$

Note that $e^{-s v \operatorname{tr}(A)}=\prod_{j=1}^{n} e^{-s v \frac{\lambda_{j}}{2}}$. It is now easy to check that

$$
f(v)=\frac{1}{(2 \pi)^{\frac{n}{2}}}\left(\prod_{j=1}^{n} \frac{v}{\sinh \left(s v \lambda_{j}\right)}\right)^{\frac{1}{2}} \in \mathcal{S}(\mathbb{R}) .
$$

The smoothness of $k(s, x, y, \xi)$ then follows by the smoothness of the function $g$. Eventhough, in all our examples the function $k(s, \cdot, y, \cdot) \in \mathcal{S}\left(\mathbb{R}^{n} \times \mathbb{R}\right)$ for every fixed pair $(s, y) \in \mathbb{R}_{+} \times \mathbb{R}^{n}$ it is not clear if this property always holds true for the heat kernel obtained in (4.3.24).

We illustrate the above technique by calculating the (well-known) heat kernels of the Hermite operator on $\mathbb{R}^{n}$ (c.f. Section 5.7 in [56]) and the isotropic twisted Laplacian on $\mathbb{R}^{2 N}$ (c.f. $[161,164])$. In the case of the Hermite operator (respectively the isotropic Laplace operator) the corresponding matrix $A$ is real (respectively Hermitian) satisfying the conditions of the above corollary.

Example 4.3.1. Let $\xi \neq 0$ be fixed and consider the Hermite operator $\mathcal{G}_{\xi}$ on $L^{2}\left(\mathbb{R}^{n}\right)$ defined by

$$
\mathcal{G}_{\xi}:=\sum_{j=1}^{n}\left(\xi^{2} x_{j}^{2}-\frac{\partial^{2}}{\partial x_{j}^{2}}\right) .
$$

Note that the operator $\mathcal{G}_{\xi}$ is of the form (4.3.23). Hence its heat kernel which is denoted by $k_{\mathcal{G}_{\xi}}(s, x, y)$ is given by (4.3.24) with $A=I d$ i.e.

$$
\begin{aligned}
& k_{\mathcal{G}_{\xi}}(s, x, y) \\
& \quad=e^{-s|\xi| n}\left(\frac{|\xi|}{\pi}\right)^{\frac{n}{2}}\left(\frac{1}{1-e^{-4 s|\xi|}}\right)^{\frac{n}{2}} \exp \left\{\frac{-|\xi|}{2} \frac{1+e^{-4 s|\xi|}}{1-e^{-4 s|\xi|}}\left(|x|^{2}+|y|^{2}\right)+2|\xi| \frac{e^{-2 s|\xi|}}{1-e^{-4 s|\xi|}} y \cdot x\right\} \\
& \quad=\left(\frac{\xi}{2 \pi \sinh (2 s \xi)}\right)^{\frac{n}{2}} e^{-\frac{\xi \operatorname{coth}(2 s \xi)}{2}\left(|x|^{2}+|y|^{2}\right)+\frac{\xi}{\sinh (2 s \xi)} y \cdot x} .
\end{aligned}
$$


Remark that when replacing $\xi$ by 0 in Equation (4.3.26) we obtain the Laplacian

$$
\Delta=-\sum_{j=1}^{n} \frac{\partial^{2}}{\partial x_{j}^{2}}
$$

on $\mathbb{R}^{n}$. Making $\xi \rightarrow 0$ in (4.3.27) provides the heat kernel of $\Delta$. Indeed, using the fact that $\lim _{\xi \rightarrow 0} \frac{\xi}{\sinh (2 s \xi)}=\frac{1}{2 s}$ we have

$$
\lim _{\xi \rightarrow 0} k_{\mathcal{G}_{\xi}}(s, x, y)=\left(\frac{1}{4 s \pi}\right)^{\frac{n}{2}} e^{-\frac{1}{4 s}|x-y|^{2}},
$$

which is the heat kernel for the Laplacian $\Delta$.

Example 4.3.2. For $\xi \neq 0$ the isotropic twisted Laplacian on $\mathbb{R}^{2 N}$ is the elliptic operator given by

$$
L_{\xi}=-\frac{1}{2}\left(\sum_{l=1}^{N}\left(\frac{\partial}{\partial x_{l}}-i \xi x_{l+N}\right)^{2}+\left(\frac{\partial}{\partial x_{l+N}}+i \xi x_{l}\right)^{2}\right) .
$$

We write the above operator in the form (4.3.10)

$$
\begin{aligned}
L_{\xi} & =-\sum_{j=1}^{n} \frac{1}{2}\left(\frac{\partial^{2}}{\partial x_{j}^{2}}-\xi^{2} x_{j}^{2}\right)-i \xi \sum_{l=1}^{N}\left(x_{l} \frac{\partial}{\partial x_{l+N}}-x_{l+N} \frac{\partial}{\partial x_{l}}\right) \\
& =\sum_{j=1}^{n} a_{j j}\left(\xi^{2} x_{j}^{2}-\frac{\partial^{2}}{\partial x_{j}^{2}}\right)-2 i \xi \operatorname{Im} \sum_{j>k} a_{j k}\left(x_{k} \frac{\partial}{\partial x_{j}}-x_{j} \frac{\partial}{\partial x_{k}}\right)
\end{aligned}
$$

where $n=2 N$ and $A=\left(a_{j k}\right)$ is the $n \times n$ Hermitian matrix given by

$$
a_{j j}=\frac{1}{2} \quad \text { for all } j=1, \cdots, n \quad \text { and } \quad a_{j k}=\frac{i}{2} \delta_{j, k+N} \quad \text { for all } j>k .
$$

Hence $A=\frac{1}{2} I d+i \operatorname{Im}(A)$ where $\operatorname{Im}(A)$ is the antisymmetric real matrix defined by $a_{j k}=$ $\frac{1}{2} \delta_{j, k+N}$ for all $j>k$. It is now obvious that $A \bar{A}=\bar{A} A$ and $A+\bar{A}=I d$ is positive definite. Moreover since $A$ is diagonally dominant this ensures that $A$ is positive semidefinite. Therefore, the heat kernel of $L_{\xi}$ is given by (4.3.24) with the above defined matrix A. Let us simplify the ingredients in (4.3.24) for the matrix A associated to $L_{\xi}$. On the one hand, it is easy to check that $A^{2}=A$ therefore the exponential matrix $e^{-2 s|\xi| A}$ is given by

$$
e^{-2 s|\xi| A}=I d+\left(e^{-2 s|\xi|}-1\right) A .
$$

This shows that the Hermitian matrix $M:=2\left(I d-e^{-2 s|\xi|(A+\bar{A})}\right)^{-1} e^{-2 s|\xi| A}=\left(m_{j k}\right)$ is given by

$$
\begin{aligned}
& m_{j j}=\frac{2}{1-e^{-2 s|\xi|}}\left(e^{-2 s|\xi|}-1\right) a_{j j}=\operatorname{coth}(s|\xi|) \quad \text { for all } j=1, \cdots, n, \quad \text { and } \\
& m_{j k}=\frac{2}{1-e^{-2 s|\xi|}}\left(e^{-2 s|\xi|}-1\right) a_{j k}=-i \delta_{j, k+N} \quad \text { for all } j>k .
\end{aligned}
$$

Therefore, for any $y=\left(y_{1}, \cdots, y_{n}\right), x=\left(x_{1}, \cdots, x_{n}\right) \in \mathbb{R}^{n}$ we have

$$
y M x=y \cdot x\left[\operatorname{coth}(s|\xi|)-i \sum_{k=1}^{N}\left(x_{k} y_{k+N}-x_{k+N} y_{k}\right)\right] .
$$


On the other hand, since $A+\bar{A}=I d$ we obtain

$$
\left(I d-e^{-2 s|\xi|(A+\bar{A})}\right)^{-1}\left(I d+e^{-2 s|\xi|(A+\bar{A})}\right)=\operatorname{coth}(s|\xi|) I d .
$$

Hence, the heat kernel of $L_{\xi}$ denoted by $k_{L_{\xi}}(s, x, y)$ is given by

$$
\begin{aligned}
k_{L_{\xi}}(s, x, y) & =\frac{1}{\pi^{N}}\left(\frac{|\xi| e^{-s|\xi|}}{1-e^{-2 s|\xi|}}\right)^{N} e^{-\frac{|\xi|}{2} \operatorname{coth}(s|\xi|)\left(|x|^{2}+|y|^{2}\right.} e^{|\xi| y M x^{T}} \\
& =\frac{1}{(2 \pi)^{N}}\left(\frac{\xi}{\sinh (s \xi)}\right)^{N} e^{-\frac{\xi}{2} \operatorname{coth}(s \xi)\left[\sum_{j=1}^{n}\left(x_{j}-y_{j}\right)^{2}\right]} e^{-i \xi \sum_{k=1}^{N}\left(x_{k} y_{k+N}-y_{k} x_{k+N}\right)}
\end{aligned}
$$

As in Example (4.3.1) we remark that replacing $\xi$ by 0 in Equation (4.3.28) yields to the Laplacian

$$
\Delta_{1}=-\frac{1}{2} \sum_{j=1}^{n} \frac{\partial^{2}}{\partial x_{j}^{2}} .
$$

Taking the limit as $\xi \rightarrow 0$ in (4.3.30) provides the heat kernel $K_{\Delta_{1}}(s, x, y)$ of $\Delta_{1}$

$$
\lim _{\xi \rightarrow 0} k_{\mathcal{G}_{\xi}}(s, x, y)=\left(\frac{1}{2 s \pi}\right)^{\frac{n}{2}} e^{-\frac{1}{2 s}|x-y|^{2}}=K_{\Delta_{1}}(s, x, y) .
$$

Finally we obtain the heat kernel of the operator (4.3.6) in the following theorem.

Theorem 4.3.7. Let $A \in M_{n}(\mathbb{C})$ to be a $n \times n$ positive semidefinite matrix such that $(A+\bar{A})$ is positive definite. Consider the differential operator $\mathcal{L}$ on $\mathbb{R}^{n+1}$ defined by

$$
\begin{gathered}
\mathcal{L}=-\sum a_{j j}\left(\frac{\partial^{2}}{\partial x_{j}^{2}}+x_{j}^{2} \frac{\partial^{2}}{\partial u^{2}}\right)-2 \operatorname{Re} \sum_{j>k} a_{j k} x_{j} x_{k} \frac{\partial^{2}}{\partial u^{2}}-2 \operatorname{Im} \sum_{j>k} a_{j k}\left(x_{k} \frac{\partial^{2}}{\partial x_{j} \partial u}-x_{j} \frac{\partial^{2}}{\partial x_{k} \partial u}\right) \\
-2 \operatorname{Re} \sum_{j>k} a_{j k} \frac{\partial^{2}}{\partial x_{k} \partial x_{j}},
\end{gathered}
$$

where $(x, u)=\left(x_{1}, \cdots, x_{n}, u\right) \in \mathbb{R}^{n} \times \mathbb{R}$. Then $\mathcal{L}$ is subelliptic positive and essentially selfadjoint as an operator on $\mathcal{S}\left(\mathbb{R}^{n+1}\right)$. Suppose that $A \bar{A}=\bar{A} A$ and for every $s>0$ the function $k(s, x, y, \xi):=k_{\xi}(s, x, y) \in C^{\infty}\left(\mathbb{R}^{2 n}\right)$ (c.f. Remark 4.3.5), where $k_{\xi}(s, x, y)$ is given by (4.3.24), satisfies the conditions in Proposition 4.3.1 (2). Then the heat kernel of $\mathcal{L}$ denoted by $k_{\mathcal{L}}(s, x, u, y, \tilde{u})$ is given by

$$
\begin{aligned}
& k_{\mathcal{L}}(s, x, u, y, \tilde{u})=\frac{1}{2 \pi} \int_{\mathbb{R}} e^{-s|\xi| \operatorname{tr}(A)}\left(\frac{|\xi|}{\pi}\right)^{\frac{n}{2}} \sqrt{\operatorname{det}\left(I d-e^{-2 s|\xi|(A+\bar{A})}\right)^{-1}} \\
& \exp \left\{\frac{-|\xi|}{2} y\left(I d-e^{-2 s|\xi|(A+\bar{A})}\right)^{-1}\left(I d+e^{-2 s|\xi|(A+\bar{A})}\right) y\right. \\
& \frac{-|\xi|}{2} x\left(I d-e^{-2 s|\xi|(A+\bar{A})}\right)^{-1}\left(I d+e^{-2 s|\xi|(A+\bar{A})}\right) x \\
&\left.+2|\xi| y\left(I d-e^{-2 s|\xi|(A+\bar{A})}\right)^{-1} e^{-2 s|\xi| A} x\right\} e^{i \xi(u-\tilde{u})} d \xi .
\end{aligned}
$$


Proof. The positivity and the sub-ellipticity of $\mathcal{L}$ was proved in Theorem 4.3.1. Let us check that $\mathcal{L}$ is essentially self adjoint as an operator on $\mathcal{S}\left(\mathbb{R}^{n+1}\right)$. Since $\mathcal{L}$ is symmetric on $\mathcal{S}\left(\mathbb{R}^{n+1}\right)$ the operator $\mathcal{L}$ can be extended to a selfadjoint operator (for example the Friedrich's extension). We will prove that $\mathcal{L}$ has a unique self adjoint extension i.e. $\mathcal{L}$ is essentially self adjoint. Let $\mathcal{L}_{1}$ and $\mathcal{L}_{2}$ be two selfadjoint extensions of $\mathcal{L}$. By Corollary 4.3.2 we know that for each $\xi \neq 0$ the operator $\mathcal{L}_{\xi}:=\mathcal{F}_{u} \mathcal{L F}_{u}{ }_{\mid(\cdot, \xi)}^{-1}$ is essentially selfadjoint on $\mathcal{D}_{\xi} \subset \mathcal{S}\left(\mathbb{R}^{n}\right)$ which shows that $\mathcal{L}_{\xi}$ is again essentially selfadjoint on $\mathcal{S}\left(\mathbb{R}^{n}\right)$. Note that $\mathcal{F}_{u} \mathcal{L}_{1} \mathcal{F}_{\left.u\right|_{(, \xi)}}^{-1}$ and $\mathcal{F}_{u} \mathcal{L}_{2} \mathcal{F}_{\left.u\right|_{(, \xi)}}^{-1}$ are selfadjoint extensions of $\left.\mathcal{L}_{\xi}\right|_{\mathcal{S}\left(\mathbb{R}^{n}\right)}$. Therefore, for any $\xi \neq 0$ we have $\mathcal{F}_{u} \mathcal{L}_{1} \mathcal{F}_{\left.u\right|_{(\cdot, \xi)}}^{-1}=\mathcal{F}_{u} \mathcal{L}_{2} \mathcal{F}_{\left.u\right|_{(\cdot, \xi)}}^{-1}$ which shows that $\mathcal{L}_{1} \mathcal{F}_{u}{ }^{-1}=\mathcal{L}_{2} \mathcal{F}_{u}{ }^{-1}$ i.e. $\mathcal{L}_{1}=\mathcal{L}_{2}$. Finally, we calculate the heat kernel of the operator $\mathcal{L}$. Again by Corollary 4.3.2 we know that the heat kernel $k_{\xi}(s, x, y)$ of the operator $\mathcal{L}_{\xi}$ is given by (4.3.24) for all $\xi \neq 0$. However, by Proposition 4.3.1 the heat kernel of the operator $\mathcal{L}$ on $\mathbb{R}^{n+1}$ is given by

$$
k_{\mathcal{L}}(s, x, u, y, \tilde{u})=\frac{1}{2 \pi} \int_{\mathbb{R}} k_{\xi}(s, x, y) e^{i \xi(u-\tilde{u})} d \xi
$$

We apply the above theorem to calculate the heat kernel of the Grusin operator on $\mathbb{R}^{n+1}$ and that of the sub-Laplacian $\Delta_{\text {sub }}$ on the $(2 N+1)$-dimensional Heisenberg group $\mathbb{H}_{(2 N+1)}$.

Example 4.3.3. The subelliptic operator

$$
\mathcal{G}:=-\sum_{j=1}^{n}\left(\frac{\partial^{2}}{\partial x_{j}^{2}}+x_{j}^{2} \frac{\partial^{2}}{\partial u^{2}}\right), \quad(x, u) \in \mathbb{R}^{n} \times \mathbb{R}
$$

is called the Grusin operator. It is easy to check that for each $\xi \neq 0$ the operator $\mathcal{G}_{\xi}:=$ $\left.\mathcal{F}_{u} \mathcal{G F}_{u}\right|_{(\cdot, \xi)} ^{-1}$ is the Hermite operator on $\mathbb{R}^{n}$ considered in Example (4.3.1) and thus its heat kernel $k_{\mathcal{G}_{\xi}}(s, x, y)$ is given by (4.3.27). Therefore, the heat kernel of the Grusin operator is obtained via the inverse Fourier transform

$$
\begin{aligned}
K_{\mathcal{G}}(s, x, u, y, \tilde{u}) & =\frac{1}{2 \pi} \int_{\mathbb{R}} k_{\mathcal{G}_{\xi}}(s, x, y) e^{i \xi(u-\tilde{u})} d \xi \\
& =\frac{1}{(2 \pi)^{1+\frac{n}{2}}} \int_{\mathbb{R}}\left(\frac{\xi}{\sinh (2 s \xi)}\right)^{\frac{n}{2}} e^{-\frac{\xi \operatorname{coth}(2 s \xi)}{2}\left(|x|^{2}+|y|^{2}\right)+\frac{\xi}{\sinh (2 s \xi)} y \cdot x} e^{i \xi(u-\tilde{u})} d \xi \\
& =\frac{1}{(2 s \pi)^{1+\frac{n}{2}}} \int_{\mathbb{R}}\left(\frac{\tau}{\sinh (2 \tau)}\right)^{\frac{n}{2}} e^{-\frac{\tau}{2 s} \operatorname{coth}(2 \tau)\left(|x|^{2}+|y|^{2}\right)+\frac{\tau}{s \sinh (2 \tau)} y \cdot x} e^{i \frac{\tau}{s}(u-\tilde{u})} d \tau,
\end{aligned}
$$

where the last equality is obtained by using the change of variable $\tau=s \xi$.

Example 4.3.4. For the sub-Laplace operator $\Delta_{\text {sub }}$ on $\mathbb{H}_{(2 N+1)}$ given by (4.2.22) we apply the 
above theorem to obtain its heat kernel. A simple computation shows that $\Delta_{\text {sub }}$ takes the form

$$
\begin{aligned}
\Delta_{\text {sub }} & =-\frac{1}{2}\left(\sum_{l=1}^{N} \frac{\partial^{2}}{\partial x_{l}^{2}}+x_{l+N}^{2} \frac{\partial^{2}}{\partial u^{2}}+\frac{\partial^{2}}{\partial x_{l+N}^{2}}+x_{l}^{2} \frac{\partial^{2}}{\partial u^{2}}+2\left(x_{l} \frac{\partial^{2}}{\partial x_{l+N} \partial u}-x_{l+N} \frac{\partial^{2}}{\partial x_{l} \partial u}\right)\right) \\
& =-\sum_{j=1}^{n} \frac{1}{2}\left(\frac{\partial^{2}}{\partial x_{j}^{2}}+x_{j}^{2} \frac{\partial^{2}}{\partial u^{2}}\right)-\sum_{l=1}^{N}\left(x_{l} \frac{\partial^{2}}{\partial x_{l+N} \partial u}-x_{l+N} \frac{\partial^{2}}{\partial x_{l} \partial u}\right) \\
& =-\sum_{j=1}^{n} a_{j j}\left(\frac{\partial^{2}}{\partial x_{j}^{2}}+x_{j}^{2} \frac{\partial^{2}}{\partial u^{2}}\right)-2 \operatorname{Im} \sum_{j>k} a_{j k}\left(x_{k} \frac{\partial^{2}}{\partial x_{j} \partial u}-x_{j} \frac{\partial^{2}}{\partial x_{k} \partial u}\right),
\end{aligned}
$$

where $n=2 N$ and $A=\left(a_{j k}\right)$ is the $n \times n$ Hermitian matrix defined by (4.3.29) in Example (4.3.2). In fact, for any $\xi \neq 0$ the operator $\mathcal{L}_{\xi}:=\mathcal{F}_{u} \Delta_{\text {sub }} \mathcal{F}_{\left.u\right|_{(\cdot, \xi)} ^{-1}}^{-1}$ is the isotropic twisted Laplace operator on $\mathbb{R}^{2 N}$ given by (4.3.28). The heat kernel of $\mathcal{L}_{\xi}$ denoted by $k_{L_{\xi}}(s, x, y)$ is given by Equation (4.3.30). This shows that the heat kernel of $\Delta_{\text {sub }}$ is given by

$$
\begin{aligned}
& k_{\Delta_{\mathrm{sub}}}(s, x, u, y, \tilde{u})=\frac{1}{2 \pi} \int_{\mathbb{R}} k_{L_{\xi}}(s, x, y) e^{i \xi(u-\tilde{u})} d \xi \\
& =\frac{1}{(2 \pi)^{N+1}} \int_{\mathbb{R}}\left(\frac{\xi}{\sinh (s \xi)}\right)^{N} e^{-\frac{\xi}{2} \operatorname{coth}(s \xi)\left[\sum_{j=1}^{n}\left(x_{j}-y_{j}\right)^{2}\right]} e^{-i \xi\left(\tilde{u}-u+\sum_{k=1}^{N}\left(x_{k} y_{k+N}-y_{k} x_{k+N}\right)\right)} d \xi \\
& =\frac{1}{(2 s \pi)^{N+1}} \int_{\mathbb{R}}\left(\frac{\tau}{\sinh \tau}\right)^{N} e^{-\frac{\tau}{2 s} \operatorname{coth} \tau\left[\sum_{j=1}^{n}\left(x_{j}-y_{j}\right)^{2}\right]} e^{-i \frac{\tau}{s}\left(\tilde{u}-u+\sum_{k=1}^{N}\left(x_{k} y_{k+N}-y_{k} x_{k+N}\right)\right)} d \tau,
\end{aligned}
$$

where in the last equality we used the change of variable $\tau=s \xi$. 


\section{Chapter 5}

\section{Compact Toeplitz operators for weighted Bergman spaces on bounded symmetric domains}

Let $\Omega \subset \mathbb{C}^{d}$ be an irreducible bounded symmetric domain of multiplicities $a$ and $b$, rank $r$ and genus $p$. For each $\nu>p-1$, we consider on $\Omega$ the normalized weighted measure $d \mu_{\nu}:=c_{\nu} h(z, z)^{\nu-p} d v$ where $h(z, w)\left(z, w \in \mathbb{C}^{d}\right)$ is the Jordan triple determinant polynomial associated to $\Omega$. In this chapter, we study Toeplitz operators $T_{g}^{\nu}$ with symbol $g$ acting on the standard weighted Bergman space $H_{\nu}^{2}:=H_{\nu}^{2}\left(\Omega, d \mu_{\nu}\right)$ over $\Omega$ with weight $\nu$. Under some conditions on the weights $\nu$ and $\nu_{0}$ we show that there exists $C\left(\nu, \nu_{0}\right)>0$, such that the Berezin transform $\tilde{g}_{\nu_{0}}$ of $g$ with respect to $H_{\nu_{0}}^{2}$ satisfies:

$$
\left\|\tilde{g}_{\nu_{0}}\right\|_{\infty} \leq C\left(\nu, \nu_{0}\right)\left\|T_{g}^{\nu}\right\|
$$

for all $g$ in a suitable class of symbols containing $L^{\infty}(\Omega)$. As a consequence we apply a result in [77], to prove that the compactness of $T_{g}^{\nu}$ is independent of the weight $\nu$, whenever $g \in L^{\infty}(\Omega)$ and $\nu>C$ where $C$ is a constant depending on $(r, a, b)$.

\subsection{Introduction}

In this chapter we switch our attention to weighted Bergman spaces over bounded symmetric domains. For an irreducible bounded symmetric domain $\Omega \subset \mathbb{C}^{d}$ of type $(r, a, b)$ in its Harish-Chandra realization we write $\langle\cdot, \cdot\rangle_{\nu}$ for the usual inner product on $L_{\nu}^{2}:=L^{2}\left(\Omega, d \mu_{\nu}\right)$ whenever $\nu>1+(r-1) a+b$. From Section 1.2 we know that the weighted Bergman space $H_{\nu}^{2}:=H_{\nu}^{2}\left(\Omega, d \mu_{\nu}\right) \subset L_{\nu}^{2}$ is closed in $L_{\nu}^{2}$ and forms a reproducing kernel Hilbert space. Via the orthogonal projection $P_{\nu}$ from $L_{\nu}^{2}$ onto $H_{\nu}^{2}$ and for a measurable symbol $f$, the Toeplitz operator $T_{f}^{\nu}$ is defined on a suitable domain in $H_{\nu}^{2}$ as the product $T_{f}^{\nu}:=P_{\nu} M_{f}$ where $M_{f}$ is the multiplication by $f$. Moreover, for a suitable function $g: \Omega \longrightarrow \mathbb{C}$ the weighted Berezin transform $\widetilde{g}_{\nu}$ is the real analytic map on $\Omega$ given by $\widetilde{g}_{\nu}(z)=\left\langle T_{g}^{\nu} k_{\nu,[z]}, k_{\nu,[z]}\right\rangle_{\nu}$ where $k_{\nu,[z]}$ denotes the normalized Bergman kernel. 
As it was mentioned in Sections 1.3 and 1.4 the Berezin transform is an important tool in the analysis of Toeplitz operators giving rise to various results in operator theory (c.f. $[10,15$, $18,35,64,77,187])$. For example, it was shown in [77] that under some condition on the weight $\nu$ the Toeplitz operator $T_{g}^{\nu}$ with bounded symbol $g$ is compact on $H_{\nu}^{2}$ if and only if $\widetilde{g}_{\nu} \in C_{0}(\Omega)$. In case of the unit disc $\Omega=\mathbb{D}$ in the complex plane, this result was generalized from bounded symbols to symbols of bounded mean oscillation $B M O^{1}(\mathbb{D})$ (c.f. [187]). The corresponding compactness characterization also holds true for weighted Bergman spaces over the n-dimensional unit ball $\Omega=\mathbb{B}^{n}$ and $g \in B M O^{1}\left(\mathbb{B}^{n}\right)$ (c.f. [15]). Theorems of the previously mentioned type have been obtained for unbounded domains, as well. In case of the SegalBargmann space $H^{2}\left(\mathbb{C}^{n}, d \mu_{t}\right)$, it was proved in [15] that for symbols $f \in B M O^{1}\left(\mathbb{C}^{n}\right)$ the Toeplitz operator $T_{f}^{t}$ is bounded (respectively compact) if and only if the heat transform $\widetilde{f}^{\left(\frac{t}{2}\right)}$ (c.f. (1.3.12) for the definition) at time $\frac{t}{2}$ is bounded (respectively vanishing at infinity) (c.f. $[15,64])$.

A natural question which arises in the study of Toeplitz operators with a fixed symbol acting on a family of weighted Bergman spaces is wether their compactness is independent of the weight parameter. By essentially using the previously mentioned results it was shown in [15] that an independence in fact holds in case of the Segal-Bargmann space $H^{2}\left(\mathbb{C}^{n}, d \mu_{t}\right)$ and the weighted Bergman spaces $A_{\lambda}^{2}$ over the unit ball $\mathbb{B}^{n}$ under the assumption that $g \in B M O^{1}\left(\mathbb{C}^{n}\right)$ and $g \in B M O^{1}\left(\mathbb{B}^{n}\right)$, respectively. However, there are counter examples for general symbols (c.f. Section 6 in [15]). As an application it follows that for functions $g \in B M O^{1}\left(\mathbb{C}^{n}\right)$ the heat transform $\widetilde{g}^{(t)} \in C\left(\mathbb{C}^{n}\right)$ of $g$ vanishes at infinity for a certain time $t_{0}>0$ if and only if $\widetilde{g}^{(t)}$ vanishes at infinity for each time $t>0$. This, roughly speaking, gives some information on the heat flow "backwards in time".

In the case of a bounded symmetric domain, the weight parameter $\nu$ replaces the time parameter $t$ in the Segal-Bargmann space construction and the Berezin transform $\widetilde{g}_{\nu}$ replaces the heat transform (1.3.12). Thus it is natural to ask whether the compactness of the Toeplitz operator $T_{g}^{\nu}$ on the standard weighted Bergman space corresponding to the weight parameter $\nu$ on a bounded symmetric domain depends on $\nu$. The aim of this chapter is to prove that the compactness of $T_{g}^{\nu}$ is uniform with respect to the weight $\nu$, whenever $g \in L^{\infty}(\Omega)$ and $\nu>C$ where $C$ is a constant depending on $(r, a, b)$.

The first attempt to solve such a problem was given in [15] for the case of the SegalBargmann space. One important ingredient was to obtain an estimation between the sup-norm of the heat transform of the symbol and the norm of the Toeplitz operator (c.f. Theorem 11 [35], Proposition 1 and Theorem 10 in [15]) and to use the above mentioned result in [187]. In this chapter, we employ a similar technique to investigate the case of a general bounded symmetric domain. In particular, for suitable weights $\nu$ and $\nu_{0}$ we give an upper estimate for the $\nu_{0}$-Berezin transform in terms of the operator norm of the Toeplitz operator $T_{g}^{\nu}$ (c.f. Theorem A below). We then use a compactness characterization in [77] which holds for bounded symbols proving the uniform compactness of $T_{g}^{\nu}$ w.r.t. the weight $\nu$ (c.f. Theorem B below). We point out that under the assumption of boundedness our result is a generalization to that in [15] since we deal 
with a wider class of domains. However, we are not able to generalize the statement to functions of bounded mean oscillation. This is due to the fact that the equivalence between the vanishing of the Berezin transform at the boundary of $\Omega$ and the compactness of Toeplitz operators is not clear for such a space of symbols.

Here we state two of our main results:

Theorem A. Let $\nu>p-1, \nu_{0}>d$ with $\left|\nu-\nu_{0}\right|>\frac{r-1}{2} a$ and write $\tilde{\nu}=\min \left\{\nu, \nu_{0}\right\}$. Then there exists $C\left(\nu, \nu_{0}\right)>0$ such that for all $g \in \tau_{\nu}(\Omega) \cap L^{1}\left(\Omega, d \mu_{\tilde{\nu}}\right)(c . f$. (5.2.5)) we have

$$
\left\|\widetilde{g}_{\nu_{0}}\right\|_{\infty} \leq C\left(\nu, \nu_{0}\right)\left\|T_{g}^{\nu}\right\|
$$

Theorem B. Let $\Omega \subset \mathbb{C}^{d}$ be an irreducible bounded symmetric domain and suppose that

$$
\nu, \nu_{0}>\max \left\{d, p-1+\frac{r-1}{4} a+\sqrt{\left(\frac{r-1}{4} a\right)^{2}+\frac{r-1}{2} a\left(\frac{r-1}{2} a+p-1\right)}\right\} .
$$

Then for any $g \in L^{\infty}(\Omega)$ we have the equivalence:

$$
T_{g}^{\nu_{0}} \text { is compact on } H_{\nu_{0}}^{2} \text { if and only if } T_{g}^{\nu} \text { is compact on } H_{\nu}^{2} \text {. }
$$

Chapter 5 is organized as follows: In Section 5.2 we set up notation and present some of the standard results concerning irreducible bounded symmetric domains. In Section 5.3 we prove the inequality in Theorem A by a technique similar to that in [35]. An essential idea is to construct for each pair $\left(\nu, \nu_{0}\right)$ of weights a certain trace class operator on $H_{\nu}^{2}$ and represent the Berezin transform of a function as an operator trace. Section 5.4 is devoted to the proof of Theorem B, where we use a result in [77]. Finally, we present some open problems which are motivated by our results.

\subsection{Preliminaries}

The objective of this section is to provide some basic facts on bounded symmetric domains as well as the weighted Bergman spaces over such domains. As bounded symmetric domains are Hermitian symmetric spaces (w.r.t. the Bergman metric) we refer the reader to Helgason's book [102] for a general theory of symmetric spaces. For an algebraic description and characterization (using Jordan triple systems) of bounded symmetric domain c.f. [123, 128, 166]. A full account for the theory of Bergman spaces over bounded symmetric domains can be found in [166] and Part III of [79].

A bounded domain $\Omega \subset \mathbb{C}^{d}$ is said to be symmetric if for every point $w \in \Omega$ there exists an involutive automorphism of $\Omega$ for which $w$ is an isolated fixed point of the automorphism function. Since $\Omega$ is bounded we can consider the ,un-weighted" Bergman space $H_{\mathrm{vol}(\Omega)^{-1}}^{2}$ (c.f. Section 1.2). Let us denote by $K(z, w)(z, w \in \Omega)$ the reproducing kernel of $H_{\mathrm{vol}(\Omega)^{-1}}^{2}$. It is well known (c.f. [79] p. 190) that the $d \times d$ matrix

$$
g_{j k}(z)=\frac{\partial^{2}}{\partial z_{j} \partial \bar{z}_{k}} \log K(z, z), \quad z \in \Omega
$$


defines a Hermitian metric on $\Omega$. This metric is called the Bergman metric and gives the usual topology on $\Omega[28,124]$. Therefore, when $\Omega$ is symmetric $(\Omega, g)$ becomes a Hermitian globally symmetric space. Hence, the set of all isometries (w.r.t $g$ ) on $\Omega$ denoted by $I(\Omega)$ acts transitively on $\Omega$ i.e. $\Omega$ is homogeneous space (c.f. Lemma 3.1 of Chapter IV in [102]). Moreover, $I(\Omega)$ is a separable locally compact transformation Lie group w.r.t. the compact open topology on $I(\Omega)$ (c.f. Lemma 3.2 of Chapter IV in [102] ). Furthermore, for any $z \in \Omega$ the isotropy group (stabilizer of $z$ ) of isometries $\tilde{k}_{z}$ is a compact subgroup of $I(\Omega)$ such that the coset $I(\Omega) / \tilde{k}_{z}$ is homeomorphic to $\Omega$ (c.f. Theorem 3.2 of Chapter II in [102]). Let us denote by $A u t(\Omega)$ the group of all biholomorphic automorphisms on $\Omega$. One can easily check that every biholomorphic function on $\Omega$ is an isometry w.r.t. the Hermitian metric $g$. Hence, Aut $(\Omega) \subset I(\Omega)$ and by Montel's theorem the automorphism group has the structure of a transformation Lie group. By a theorem of E. Cartan Aut $(\Omega)$ acts transitively on $\Omega$ (c.f. [57]). Again by Theorem 3.2 of Chapter II in [102] the coset $A u t(\Omega) / k_{z}$ is homeomorphic to $\Omega$ where $k_{z}$ is the isotropy group in $\operatorname{Aut}(\Omega)$ fixing z. Moreover, if $G$ is the connected component of $A u t(\Omega)$ containing the identity then by Proposition 4.3 of Chapter II in [102] $G$ is a semi-simple Lie group. Furthermore, if $K$ is the isotropy subgroup of $G$ fixing $z$ then $K$ is a maximal compact subgroup of $G$ and $G / K \cong \Omega$ (c.f. $[102,138]$ ).

Let us consider an irreducible bounded symmetric domain $\Omega \subset \mathbb{C}^{d}$ i.e. $\Omega$ is not a Cartesian product of lower dimensional symmetric spaces. In [57], E. Cartan proved that there exist only six types of irreducible bounded symmetric domains, the so called four classical domains and two exceptional domains of dimensions 16 and 27, respectively (see also Chapter IX in [102] for the classification of irreducible globally symmetric spaces). There is also a complete algebraic classification of bounded symmetric domains using Jordan systems (c.f. Chapter I in [166]). More precisely, we consider $\Omega \subset \mathbb{C}^{d}$ in its (Harish-Chandra) realization with multiplicities $a$ and $b$ and with rank $r$. In particular, $\Omega$ contains the origin and it is invariant under the natural $\mathbb{S}^{1}$-action (circular). The triple $(r, a, b)$ is called the type of $\Omega$ and it determines the domain up to biholomorphic equivalence (c.f. [57, 128]). Moreover, the genus $p$ of $\Omega$ and the complex dimension $d$ are given by:

$$
p=2+(r-1) a+b ; \quad d:=\operatorname{dim} \Omega=r+\frac{\operatorname{ar}(r-1)}{2}+r b .
$$

To each such $\Omega$ there is attached a unique function $h(z, w)\left(z, w \in \mathbb{C}^{d}\right)$ which is a polynomial in $z$ and $\bar{w}$, called the Jordan triple determinant and satisfies the following properties (c.f. $[128,166])$ :

1. $h(z, 0)=1$ and $h(z, w)=\overline{h(w, z)}, \quad \forall z, w \in \mathbb{C}^{d}$.

2. $h(z, z)>0, \quad \forall z \in \Omega$ and $h(z, z)=0, \quad \forall z \in \partial \Omega$.

Thus for any $\lambda \in \mathbb{R}$, we fix a branch of $h(z, w)^{\lambda}$ for $z, w \in \Omega$. It is well known that for any $\nu \in \mathbb{R}$ (c.f. [166]):

$$
\int_{\Omega} h(z, z)^{\nu-p} d v(z)<\infty \Longleftrightarrow \nu>p-1
$$


where $d v(z)$ is the Euclidean measure on $\Omega$. In this case, we consider the normalized weighted measure:

$$
d \mu_{\nu}=c_{\nu} h(z, z)^{\nu-p} d v
$$

The weighted Bergman space $H_{\nu}^{2}$ is the space of holomorphic functions in $L_{\nu}^{2}$. We write $K_{\nu}$ : $\Omega \times \Omega \longrightarrow \mathbb{C}$ for the reproducing kernel of $H_{\nu}^{2}$, i.e. for any $f \in H_{\nu}^{2}, w \in \Omega$ we have:

$$
f(w)=\left[P_{\nu} f\right](w)=\left\langle f, K_{\nu}(\cdot, w)\right\rangle_{\nu}=\left\langle f, K_{\nu,[w]}\right\rangle_{\nu}
$$

where $P_{\nu}$ denotes the projection of $L_{\nu}^{2}$ onto $H_{\nu}^{2}$ and $K_{\nu,[w]}:=K_{\nu}(\cdot, w)$. The kernel $K_{\nu}(z, w)$ is related to the Jordan triple determinant $h(z, w)$ via (c.f. [80]):

$$
K_{\nu}(z, w)=h(z, w)^{-\nu}, \quad \forall \nu>p-1
$$

For a measurable symbol $g: \Omega \longrightarrow \mathbb{C}$ the Toeplitz operator $T_{g}^{\nu}$ is given by:

$$
T_{g}^{\nu}: D\left(T_{g}^{\nu}\right):=\left\{h \in H_{\nu}^{2} \mid g h \in L_{\nu}^{2}\right\} \subset H_{\nu}^{2} \longrightarrow H_{\nu}^{2}: h \mapsto P_{\nu}(g h) .
$$

The Berezin transform $\widetilde{T}$ of an operator $T$ on $H_{\nu}^{2}$ with the domain of $T$ containing all the normalized kernels $k_{\nu,[z]}:=K_{\nu,[z]} /\left\|K_{\nu,[z]}\right\|_{\nu}$ where $z$ runs through $\Omega$, is the complex valued map defined on $\Omega$ by:

$$
\widetilde{T}(z)=\left\langle T k_{\nu,[z]}, k_{\nu,[z]}\right\rangle_{\nu}, \quad z \in \Omega
$$

In the rest of the chapter we will frequently use the symbol space:

$$
\tau_{\nu}(\Omega):=\left\{g: \Omega \longrightarrow \mathbb{C} \mid g K_{\nu,[a]} \in L^{2}\left(\Omega, d \mu_{\nu}\right) \text { for all } a \in \Omega\right\} \text {. }
$$

Note that for $g \in \tau_{v}(\Omega)$ the operator $T_{g}^{\nu}$ is densely defined on $H_{\nu}^{2}$ and its Berezin transform is defined to be:

$$
\widetilde{g}_{\nu}:=\widetilde{T_{g}^{\nu}}
$$

Let $P$ be the space of all holomorphic polynomials on $\mathbb{C}^{d}$. For $f, g \in P$ we define $\left.g^{*}(z):=\overline{g(\bar{z}}\right)$ and $\partial_{f}:=f\left(\frac{\partial}{\partial z}\right)$. Let $d v(z)$ denote the Lebesgue volume measure, and equip $P$ with the Fischer inner product:

$$
\langle f, g\rangle_{F}:=\partial_{f}\left(g^{*}\right)(0)=\pi^{-d} \int_{\mathbb{C}^{d}} f(z) \overline{g(z)} e^{-|z|^{2}} d v(z) .
$$

Due to the action of $K$ (the isotropy group fixing the origin) on $P$ which is given by: $\pi(k) f:=$ $f \circ k$ (each $k \in K$ can be extended to a linear map on $\mathbb{C}^{d}$ (c.f. [138])) , $P$ admits a Peter Weyl decomposition:

$$
P=\oplus P_{m}
$$

where $m=\left(m_{1}, \cdots, m_{r}\right) \in \mathbb{N}_{0}^{r}$ with $m_{1} \geq \cdots \geq m_{r} \geq 0$ (in all what follows the multiindices $m$ will always have this ordering). Note that each $P_{m}$ is a subspace of $P_{|m|}$, the space all homogeneous polynomials on $\mathbb{C}^{d}$ of degree $|m|$ (c.f. [80]).

The importance of this decomposition is given by the following theorem: 
Theorem 5.2.1 ([5]). The spaces $P_{m}$ are $K$-invariant irreducible and orthogonal under $\langle\cdot, \cdot\rangle_{F}$. Moreover, if $H$ is a Hilbert space of analytic functions on $\Omega$ with K-invariant inner product $\langle\cdot, \cdot\rangle$, then $P_{m}$ is orthogonal to $P_{n}$ under $\langle\cdot, \cdot\rangle$ whenever $m \neq n$. Moreover, $\langle\cdot, \cdot\rangle$ is proportional to $\langle\cdot, \cdot\rangle_{F}$ on each $P_{m}$.

In particular, this holds for the space $H_{\nu}^{2}$ and the constant of proportionality was calculated in [80]:

$$
\langle f, g\rangle_{F}=(\nu)_{m}\langle f, g\rangle_{\nu}
$$

where $(\nu)_{m}$ is the generalized Pochhammer symbol:

$$
(\nu)_{m}:=\prod_{j=1}^{r} \frac{\Gamma\left(m_{j}+\nu-\frac{j-1}{2} a\right)}{\Gamma\left(\nu-\frac{j-1}{2} a\right)} .
$$

Since each $P_{m}$ is closed in $P\left(P_{m}\right.$ is finite dimensional), it admits a reproducing kernel $K^{m}(z, w)$, i.e.

$$
f(w)=\left\langle f, K^{m}(\cdot, w)\right\rangle_{F}, \quad \forall f \in P_{m} .
$$

The relation between these reproducing kernels and the Bergman kernel was given in [80]:

Theorem 5.2.2 ([80]). For all $\nu \in \mathbb{C}$ and all $z, w \in \Omega$, we have:

$$
h(z, w)^{-\nu}=\sum_{m_{1} \geq \cdots \geq m_{r} \geq 0}(\nu)_{m} K^{m}(z, w) .
$$

The series converges uniformly and absolutely on compact subsets of $\Omega \times \bar{\Omega}$.

Finally, we relate the orthonormal basis of the Fischer norm to the orthonormal basis of $H_{\nu}^{2}$ : Proposition 5.2.1. Let $\nu>p-1, d_{m}=\operatorname{dim} P_{m}$, and $\left\{\psi_{j}^{m}\right\}_{j=1, \cdots, d_{m}}$ be an orthonormal basis of $\left(P_{m},\langle\cdot, \cdot\rangle_{F}\right)$. Then

$$
\mathcal{B}:=\left\{e_{j}^{m}:=(\nu)_{m}^{\frac{1}{2}} \psi_{j}^{m} \mid m \text { and } j=1, \cdots, d_{m}\right\}
$$

is an orthonormal basis of $\left(H_{\nu}^{2},\langle\cdot, \cdot\rangle_{\nu}\right)$. Here $m$ runs through the previously mentioned ordering.

Proof. Let $j \neq k$ then $\left\langle\psi_{j}^{m}, \psi_{k}^{m}\right\rangle_{\nu}=\frac{1}{(\nu)_{m}}\left\langle\psi_{j}^{m}, \psi_{k}^{m}\right\rangle_{F}=0$. Moreover, for $m \neq n$ (c.f. Theorem 5.2.1)

$$
\left\langle\psi_{j}^{m}, \psi_{i}^{n}\right\rangle_{\nu}=0 ; \quad \forall j \leq d_{m}, i \leq d_{n}
$$

Also, note that:

$$
\left\langle(\nu)_{m}^{\frac{1}{2}} \psi_{j}^{m},(\nu)_{m}^{\frac{1}{2}} \psi_{j}^{m}\right\rangle_{\nu}=(\nu)_{m}\left\langle\psi_{j}^{m}, \psi_{j}^{m}\right\rangle_{\nu}=\left\langle\psi_{j}^{m}, \psi_{j}^{m}\right\rangle_{F}=1 .
$$

Now, since the polynomials are dense in $H_{\nu}^{2}$ it follows that the functions in $\mathcal{B}$ form a complete orthonormal system of $H_{\nu}^{2}$. 


\subsection{Upper estimation of the Berezin transform}

In this section we prove Theorem A using a similar technique to that in [35]. Under the assumptions on the weights $\nu$ and $\nu_{0}$ and for each $z \in \Omega$, we find a trace class operator $T_{z}^{\nu, \nu_{0}}$ on $H_{\nu}^{2}$ where $\left\|T_{z}^{\nu, \nu_{0}}\right\|_{t r}$ is independent of $z$ and one has $\operatorname{tr}\left(T_{g}^{\nu} T_{z}^{\nu, \nu_{0}}\right)=\frac{c_{\nu}}{c_{\nu_{0}}} \widetilde{g}_{\nu_{0}}(z)$. We then apply a standard estimate for the trace norm to obtain the inequality (5.1.1).

For $X \in \mathcal{L}\left(H_{\nu}^{2}\right)$ define the function $K_{\nu}^{X}(z, w):=\left(X^{*} K_{\nu}(\cdot, w)\right)(z)$ on $\Omega \times \Omega$. We write Proposition A.1.3 for the case of the weighted Bergman space $H_{\nu}^{2}$ using the notation followed in this chapter.

Proposition 5.3.1. Let $g \in \tau_{\nu}(\Omega)$ and $X \in \mathcal{L}\left(H_{\nu}^{2}\right)$ with $\nu>p-1$. Suppose the following conditions hold:

1. $T_{g}^{\nu} \in \mathcal{L}\left(H_{\nu}^{2}\right)$.

2. $T_{g}^{\nu} X$ is of trace class on $H_{\nu}^{2}$.

3. $\int_{\Omega} \int_{\Omega}|g(z)|\left|K_{\nu}(w, z)\right|\left|K_{\nu}^{X}(w, z)\right| d \mu_{\nu}(z) d \mu_{\nu}(w)<\infty$.

Then

$$
\operatorname{tr}\left(T_{g}^{\nu} X\right)=\int_{\Omega} g(z) \overline{K_{\nu}^{X}(z, z)} d \mu_{\nu}(z)
$$

The basic idea in the proof of Theorem A is to construct a trace class operator $X=T_{0}^{\nu, \nu_{0}}$ on $H_{\nu}^{2}$ satisfying $K_{\nu}^{X}(z, w)=h(z, w)^{\nu_{0}-\nu}$. In order to do that, we fix $\left\{\psi_{j}^{m}\right\}_{j=1, \cdots, d_{m}}$ as an orthonormal basis of $\left(P_{m},\langle\cdot, \cdot\rangle_{F}\right)$. Then, the reproducing kernel $K^{m}(z, w)$ of $P_{m}$ is given by:

$$
K^{m}(z, w)=\sum_{j=1}^{d_{m}} \psi_{j}^{m}(z) \overline{\psi_{j}^{m}(w)} .
$$

Now, for each $m \in \mathbb{N}_{0}^{r}$ such that $m_{1} \geq \cdots \geq m_{r} \geq 0$ and each $j=1, \cdots, d_{m}$, we denote by $P_{j}^{m}$ the orthogonal projection from $H_{\nu}^{2}$ to the one-dimensional space spanned by $e_{j}^{m}$ :

$$
P_{j}^{m} f:=\left\langle f, e_{j}^{m}\right\rangle_{\nu} e_{j}^{m} \quad f \in H_{\nu}^{2}
$$

Hence:

$$
\begin{aligned}
\overline{K_{\nu}^{P_{j}^{m}}(z, w)}=\left[P_{j}^{m} K_{\nu}(\cdot, z)\right](w) & =\left\langle K_{\nu}(\cdot, z), e_{j}^{m}\right\rangle_{\nu} e_{j}^{m}(w) \\
& =(\nu)_{m} \overline{\psi_{j}^{m}(z)} \psi_{j}^{m}(w)
\end{aligned}
$$

Now, define the operator $T_{0}^{\nu, \nu_{0}}$ on $H_{\nu}^{2}$ as the infinite sum:

$$
T_{0}^{\nu, \nu_{0}}:=\sum_{m_{1} \geq \cdots \geq m_{r} \geq 0} \frac{\left(\nu-\nu_{0}\right)_{m}}{(\nu)_{m}} \sum_{j=1}^{d_{m}} P_{j}^{m} .
$$

Theorem 5.3.1. For $\nu>p-1$ and $\nu_{0}>d$ the operator $T_{0}^{\nu, \nu_{0}}$ is of trace class on $H_{\nu}^{2}$ and

$$
K_{\nu}^{T_{0}^{\nu, \nu_{0}}}(z, w)=h(z, w)^{\nu_{0}-\nu}
$$


Proof. For each $l \in \mathbb{N}$, consider the operator

$$
S_{l}^{\nu, \nu_{0}}:=\sum_{m_{1} \geq \cdots \geq m_{r} \geq 0,|m| \leq l} \frac{\left(\nu-\nu_{0}\right)_{m}}{(\nu)_{m}} \sum_{j=1}^{d_{m}} P_{j}^{m} .
$$

We write :

$$
\frac{\left(\nu-\nu_{0}\right)_{m}}{(\nu)_{m}}=\prod_{j=1}^{r} \frac{\Gamma\left(m_{j}+\nu-\nu_{0}-\frac{j-1}{2} a\right)}{\Gamma\left(\nu-\nu_{0}-\frac{j-1}{2} a\right)} \frac{\Gamma\left(\nu-\frac{j-1}{2} a\right)}{\Gamma\left(m_{j}+\nu-\frac{j-1}{2} a\right)} .
$$

Therefore, by Stirling's and as $m_{j} \longrightarrow \infty$ we have:

$$
\frac{\Gamma\left(m_{j}+\nu-\nu_{0}-\frac{j-1}{2} a\right)}{\Gamma\left(m_{j}+\nu-\frac{j-1}{2} a\right)} \sim m_{j}^{-\nu_{0}} .
$$

Since $\nu_{0}>p-1 \geq 1$, it follows that the operators $S_{l}^{\nu, \nu_{0}}$ converge to $T_{0}^{\nu, \nu_{0}}$ in the norm of $\mathcal{L}\left(H_{\nu}^{2}\right)$. Moreover, by an application of the Cauchy-Schwarz Theorem we know that:

$$
K_{\nu}^{S_{l}^{\nu, \nu_{0}}}(z, w) \longrightarrow K_{\nu}^{T_{0}^{\nu, \nu_{0}}}(z, w)
$$

as $l \longrightarrow \infty$ uniformly on compact subsets of $\Omega \times \Omega$ (c.f. Proposition 3-(7) in [35]). Together with Theorem 5.2.2 and the equalities (5.2.6), (5.3.2), and (5.3.3) we obtain:

$$
\begin{aligned}
K_{\nu}^{T_{0}^{\nu, \nu_{0}}}(z, w) & =\sum_{m_{1} \geq \cdots \geq m_{r} \geq 0} \frac{\left(\nu-\nu_{0}\right)_{m}}{(\nu)_{m}} \sum_{j=1}^{d_{m}} K_{\nu}^{P_{j}^{m}}(z, w) \\
& =\sum_{m_{1} \geq \cdots \geq m_{r} \geq 0} \frac{\left(\nu-\nu_{0}\right)_{m}}{(\nu)_{m}} \sum_{j=1}^{d_{m}}(\nu)_{m} \psi_{j}^{m}(z) \overline{\psi_{j}^{m}(w)} \\
& =\sum_{m_{1} \geq \cdots \geq m_{r} \geq 0}\left(\nu-\nu_{0}\right)_{m} K^{m}(z, w) \\
& =h(z, w)^{\nu_{0}-\nu}
\end{aligned}
$$

In order to prove that $T_{0}^{\nu, \nu_{0}}$ is of trace class, note that $\left\|\sum_{j=1}^{d_{m}} P_{j}^{m}\right\|_{\operatorname{tr}}=d_{m}=\operatorname{dim} P_{m}$. One has the inclusion $P_{m} \subset P_{|m|}$ and $P_{|m|}$ admits $\left\{c_{\alpha} z^{\alpha}|| \alpha|=| m \mid, \alpha \in \mathbb{N}_{0}^{d}\right\}$ (c $c_{\alpha}$ are normalized constants) as an orthonormal basis with respect to the Fischer inner product. Hence it follows that

$$
d_{m} \leq \frac{(|m|+d-1) !}{|m| !(d-1) !}
$$

Therefore,

$$
\begin{aligned}
\left\|T_{0}^{\nu, \nu_{0}}\right\|_{t r} & =\left|\sum_{m_{1} \geq \cdots \geq m_{r} \geq 0} d_{m} \prod_{j=1}^{r} \frac{\Gamma\left(m_{j}+\nu-\nu_{0}-\frac{j-1}{2} a\right)}{\Gamma\left(\nu-\nu_{0}-\frac{j-1}{2} a\right)} \frac{\Gamma\left(\nu-\frac{j-1}{2} a\right)}{\Gamma\left(m_{j}+\nu-\frac{j-1}{2} a\right)}\right| \\
& \leq \sum_{m \in \mathbb{N}^{r}} \frac{(|m|+d-1) !}{|m| !(d-1) !} \prod_{j=1}^{r}\left|\frac{\Gamma\left(m_{j}+\nu-\nu_{0}-\frac{j-1}{2} a\right)}{\Gamma\left(\nu-\nu_{0}-\frac{j-1}{2} a\right)} \frac{\Gamma\left(\nu-\frac{j-1}{2} a\right)}{\Gamma\left(m_{j}+\nu-\frac{j-1}{2} a\right)}\right| .
\end{aligned}
$$


Now, as $|m| \rightarrow \infty$, and by Stirling's formula again we have:

$$
\begin{aligned}
\frac{(|m|+d-1) !}{|m| !(d-1) !} \sim \frac{|m|^{d-1}}{(d-1) !} & =\frac{1}{(d-1) !}\left(\sum_{j=1}^{r} m_{j}\right)^{d-1} \\
& =\sum_{|\gamma|=d-1} \frac{1}{\gamma !} m^{\gamma} \leq c \prod_{i=1}^{r}\left(1+m_{i}\right)^{d-1}
\end{aligned}
$$

where $c>0$ is a suitable constant independent of $m$. Therefore, as $m_{j} \rightarrow \infty$ it follows that:

$$
\begin{gathered}
\prod_{j=1}^{r} \frac{\Gamma\left(m_{j}+\nu-\nu_{0}-\frac{j-1}{2} a\right)}{\Gamma\left(m_{j}+\nu-\frac{j-1}{2} a\right)} \frac{(|m|+d-1) !}{(|m|) !(d-1) !} \\
\leqslant c \prod_{j=1}^{r} \frac{\Gamma\left(m_{j}+\nu-\nu_{0}-\frac{j-1}{2} a\right)}{\Gamma\left(m_{j}+\nu-\frac{j-1}{2} a\right)}\left(1+m_{j}\right)^{d-1} \sim c \prod_{j=1}^{r}\left(1+m_{j}\right)^{-\nu_{0}+d-1} .
\end{gathered}
$$

Since $\nu_{0}>d$ this shows that $T_{0}^{\nu, \nu_{0}}$ is of trace class.

We want to apply Proposition 5.3.1 to $X=T_{0}^{\nu, \nu_{0}}$ and in order to check condition (3) therein, we need the generalized Forelli-Rudin inequalities which can be found in [77, 80]:

Lemma 5.3.1. Consider the integral

$$
J_{\lambda, \gamma}(z)=\int_{\Omega}|h(z, w)|^{-(\lambda+\gamma)} h(w, w)^{\lambda-p} d v(w),
$$

where $z \in \Omega, \gamma \in \mathbb{R}$ and $\lambda>p-1$. Let

$$
\mathbb{A}_{\lambda, \gamma}=\left\{\alpha \in \mathbb{R} \mid \exists C>0 \text { s.t. }\left|J_{\lambda, \gamma}(z)\right| \leq C h(z, z)^{-\alpha}, \text { for all } z \in \Omega\right\},
$$

then:

1. $\mathbb{A}_{\lambda, \gamma}=[\gamma, \infty)$, if $\gamma>\frac{r-1}{2} a$.

2. $\mathbb{A}_{\lambda, \gamma}=[0, \infty)$, if $\gamma<-\frac{r-1}{2} a$.

According to Theorem 5.3.1 and to the above lemma, we are able now to establish the following theorem:

Theorem 5.3.2. Let $\nu>p-1, \nu_{0}>d$ with $\left|\nu-\nu_{0}\right|>\frac{r-1}{2} a$, and write $\tilde{\nu}=\min \left\{\nu, \nu_{0}\right\}$. Moreover, suppose that $g \in \tau_{\nu}(\Omega) \cap L^{1}\left(\Omega, d \mu_{\tilde{\nu}}\right)$ and that $T_{g}^{\nu}$ is bounded. Then

$$
\operatorname{tr}\left(T_{g}^{\nu} T_{0}^{\nu, \nu_{0}}\right)=\frac{c_{\nu}}{c_{\nu_{0}}} \widetilde{g}_{\nu_{0}}(0)
$$

Proof. The first step is to verify condition (3) of Proposition 5.3.1 i.e. to prove the convergence of the following integral

$$
\int_{\Omega} \int_{\Omega}|g(z)|\left|K_{\nu}(w, z)\right|\left|K_{\nu}^{T_{0}^{\nu, \nu_{0}}}(w, z)\right| d \mu_{\nu}(z) d \mu_{\nu}(w) .
$$


Using (5.2.3) and (5.3.4) we obtain:

$$
\begin{gathered}
\int_{\Omega} \int_{\Omega}|g(z)|\left|K_{\nu}(w, z)\right|\left|K_{\nu}^{T_{0}^{\nu, \nu_{0}}}(w, z)\right| d \mu_{\nu}(z) d \mu_{\nu}(w) \\
=c_{\nu, \nu_{0}} \int_{\Omega} \int_{\Omega}|h(w, z)|^{-\left(\nu+\left(\nu-\nu_{0}\right)\right)} h(w, w)^{\nu-p} d v(w)|g(z)| h(z, z)^{\nu-p} d v(z) .
\end{gathered}
$$

Now, we apply Lemma 5.3.1 for $\lambda=\nu$ and $\gamma=\nu-\nu_{0}$. Since $|\gamma|>\frac{r-1}{2} a$, we have two case:

(a) In case $\nu>\nu_{0}$, Lemma 5.3.1 (1) implies that $\nu-\nu_{0} \in \mathbb{A}_{\lambda, \gamma}$, which means that:

$$
\int_{\Omega}|h(w, z)|^{-\left(\nu+\left(\nu-\nu_{0}\right)\right)} h(w, w)^{\nu-p} d v(w) \leq C h(z, z)^{\nu_{0}-\nu} .
$$

So, we have to check if

$$
\int_{\Omega} h(z, z)^{\nu_{0}-\nu} h(z, z)^{\nu-p}|g(z)| d v(z)=\frac{1}{c_{\nu_{0}}} \int_{\Omega}|g(z)| d \mu_{\nu_{0}}(z)<\infty .
$$

This is true since $g \in L^{1}\left(\Omega, d \mu_{\nu_{0}}\right)$.

(b) In case of $\nu_{0}>\nu$, we have $\gamma<-\frac{r-1}{2} a$. By Lemma 5.3.1 (2), we know that $0 \in \mathbb{A}_{\lambda, \gamma}$ i.e.

$$
\int_{\Omega}|h(w, z)|^{-\left(\nu+\left(\nu-\nu_{0}\right)\right)} h(w, w)^{\nu-p} d v(w) \leq C .
$$

Therefore, we have to investigate

$$
\int_{\Omega}|g(z)| h(z, z)^{\nu-p} d v(z)<\infty .
$$

But this is also true since in this case we have $g \in L^{1}\left(\Omega, d \mu_{\nu}\right)$.

Hence, we can apply Proposition 5.3.1 with $X=T_{0}^{\nu, \nu_{0}}$ to obtain:

$$
\begin{aligned}
\operatorname{tr}\left(T_{g}^{\nu} T_{0}^{\nu, \nu_{0}}\right) & =\int_{\Omega} g(z) \overline{K_{\nu}^{T_{0}^{\nu, \nu}}(z, z)} d \mu_{\nu}(z) \\
& =c_{\nu} \int_{\Omega} g(z) h(z, z)^{\nu_{0}-\nu} h(z, z)^{\nu-p} d v(z) \\
& =\frac{c_{\nu}}{c_{\nu_{0}}} \int_{\Omega} g(z) d \mu_{\nu_{0}}(z)=\frac{c_{\nu}}{c_{\nu_{0}}} \tilde{g}_{\nu_{0}}(0) .
\end{aligned}
$$

Our aim now is to extend the above Theorem from $z=0$ to an arbitrary $z \in \Omega$. More precisely, under the assumptions of Theorem 5.3.2 and for each $z \in \Omega$ we find a trace class operator $T_{z}^{\nu, \nu_{0}}$ such that $\operatorname{tr}\left(T_{g}^{\nu} T_{z}^{\nu, \nu_{0}}\right)=\frac{c_{\nu}}{c_{\nu_{0}}} \widetilde{g}_{\nu_{0}}(z)$ and the trace norm $\left\|T_{z}^{\nu, \nu_{0}}\right\|_{t r}=\left\|T_{0}^{\nu, \nu_{0}}\right\|_{t r}$ is independent of $z \in \Omega$.

For each $z \in \Omega$ we consider the automorphism $\phi_{z}$ which interchanges $z$ and zero. We define the linear operator $U_{\nu, z}$ on $H_{\nu}^{2}$ by

$$
U_{\nu, z} f:=\left(f \circ \phi_{z}\right) \cdot J \phi_{z}{ }^{\frac{\nu}{p}}, \quad \forall f \in H_{\nu}^{2},
$$

where $J \phi_{z}$ denotes the complex Jacobian of $\phi_{z}$.

Using the transformation formula of the unweighted Bergman kernel, one can prove: 
Proposition 5.3.2. For all $z \in \Omega$, the operator $U_{\nu, z}$ is self-adjoint and unitary i.e.

$$
U_{\nu, z}^{-1}=U_{\nu, z}^{*}=U_{\nu, z}
$$

For each $S \in \mathcal{L}\left(H_{\nu}^{2}\right)$, and each $z \in \Omega$ we define $S_{\nu, z} \in \mathcal{L}\left(H_{\nu}^{2}\right)$ by:

$$
S_{\nu, z}:=U_{\nu, z} S U_{\nu, z}
$$

The following lemma is found in ([77], Lemma 4):

Lemma 5.3.2. For all $a, z \in \Omega$, the Berezin transform $\widetilde{S_{\nu, a}}$ of $S_{\nu, a}$ is given by:

$$
\widetilde{S_{\nu, a}}(z)=\widetilde{S}\left(\phi_{a}(z)\right)
$$

One can easily check a slightly more general version of Lemma 6 in [77]:

Lemma 5.3.3. Let $\nu>p-1$ then for any $g \in \tau_{\nu}(\Omega)$ and any $a \in \Omega$ we have:

$$
U_{\nu, a} T_{g}^{\nu} U_{\nu, a}=T_{g \circ \phi_{a}}^{\nu}
$$

where both sides are interpreted as operators with domain $D\left(T_{g \circ \phi_{a}}^{\nu}\right)(c . f .(5.2 .4))$.

According to (5.3.6), we know that for any $z \in \Omega$ the operator

$$
T_{z}^{\nu, \nu_{0}}:=U_{\nu, z} T_{0}^{\nu, \nu_{0}} U_{\nu, z}
$$

is of trace class and satisfies $\left\|T_{z}^{\nu, \nu_{0}}\right\|_{t r}=\left\|T_{0}^{\nu, \nu_{0}}\right\|_{t r}$. Moreover, according to Theorem 5.3.2 together with (5.3.7) and (5.3.8) we obtain:

Corollary 5.3.1. Let $\nu>p-1$ and $\nu_{0}>d$ with $\left|\nu-\nu_{0}\right|>\frac{r-1}{2} a$. Moreover, suppose that $g \in \tau_{\nu}(\Omega) \cap L^{1}\left(\Omega, d \mu_{\tilde{\nu}}\right)$, where $\tilde{\nu}=\min \left\{\nu, \nu_{0}\right\}$, and that $T_{g}^{\nu}$ is bounded. Then we have:

$$
\operatorname{tr}\left(T_{g}^{\nu} T_{z}^{\nu, \nu_{0}}\right)=\frac{c_{\nu}}{c_{\nu_{0}}} \widetilde{g}_{\nu_{0}}(z) \quad \forall z \in \Omega
$$

Proof. We apply Theorem 5.3.2:

$$
\begin{aligned}
\operatorname{tr}\left(T_{g}^{\nu} T_{z}^{\nu, \nu_{0}}\right) & =\operatorname{tr}\left(T_{g}^{\nu} U_{\nu, z} T_{0}^{\nu, \nu_{0}} U_{\nu, z}\right)=\operatorname{tr}\left(U_{\nu, z} T_{g}^{\nu} U_{\nu, z} T_{0}^{\nu, \nu_{0}}\right) \\
& =\operatorname{tr}\left(T_{g \circ \phi_{z}}^{\nu} T_{0}^{\nu, \nu_{0}}\right)=\frac{c_{\nu}}{c_{\nu_{0}}}\left(\widehat{g \circ \phi_{z}}\right)_{\nu_{0}}(0) \\
& =\frac{c_{\nu}}{c_{\nu_{0}}} \widetilde{g}_{\nu_{0}}\left(\phi_{z}(0)\right)=\frac{c_{\nu}}{c_{\nu_{0}}} \widetilde{g}_{\nu_{0}}(z) .
\end{aligned}
$$

Proof of Theorem A: Let $T_{g}^{\nu}$ be bounded, then by Corollary 5.3.1 and by a standard estimate for the trace norm we have for all $z \in \Omega$ :

$$
\begin{aligned}
\left|\tilde{g}_{\nu_{0}}(z)\right| & \leq C_{1}\left(\nu, \nu_{0}\right)\left|\operatorname{tr}\left(T_{g}^{\nu} T_{z}^{\nu, \nu_{0}}\right)\right| \\
& \leq C_{1}\left(\nu, \nu_{0}\right)\left\|T_{g}^{\nu}\right\|\left\|T_{z}^{\nu, \nu_{0}}\right\|_{t r} \\
& =C_{1}\left(\nu, \nu_{0}\right)\left\|T_{g}^{\nu}\right\|\left\|T_{0}^{\nu, \nu_{0}}\right\|_{t r} \\
& \leq C\left(\nu, \nu_{0}\right)\left\|T_{g}^{\nu}\right\|,
\end{aligned}
$$


where $C_{1}\left(\nu, \nu_{0}\right)$ and $C\left(\nu, \nu_{0}\right)$ are suitable constants independent of $g$ and $z$. Therefore:

$$
\left\|\tilde{g}_{\nu_{0}}\right\|_{\infty} \leq C\left(\nu, \nu_{0}\right)\left\|T_{g}^{\nu}\right\|
$$

Clearly, the trace norm $\left\|T_{0}^{\nu, \nu_{0}}\right\|_{t r}$ also depends on $r$ and $a$. However, we omit indicating this fact in the constant $C\left(\nu, \nu_{0}\right)$.

\subsection{Compactness criteria}

In the following, we establish the equivalence stated in Theorem B. For this, we need a density result for a class of Toeplitz operators together with Theorem A and the main result in [77].

Let us write Theorem 1.2.2 for the case of the weighted Bergman space $H^{2}\left(\Omega, d \mu_{\nu}\right)$ using the notation followed in this chapter.

Theorem 5.4.1. Let $\nu>p-1$, then

$$
\left\{T_{g}^{\nu} \mid \text { g continuous with compact support in } \Omega\right\}
$$

is norm dense in the space of all compact operators acting on $H^{2}\left(\Omega, d \mu_{\nu}\right)$.

In order to prove Theorem $\mathrm{B}$, we use the equivalence between the compactness of the Toeplitz operator and the vanishing of the Berezin transform at the boundary of a bounded symmetric domain (c.f. Theorem A in [77]):

Theorem 5.4.2 ([77]). Let

$$
\nu>p-1+\frac{r-1}{4} a+\sqrt{\left(\frac{r-1}{4} a\right)^{2}+\frac{r-1}{2} a\left(\frac{r-1}{2} a+p-1\right)} .
$$

Denote by $C_{0}(\Omega)$ the space of functions vanishing on $\partial \Omega$. Then for any $g \in L^{\infty}(\Omega)$ the following two conditions are equivalent:

1. $T_{g}^{\nu}$ is compact on $H_{\nu}^{2}$.

2. $\widetilde{g}_{\nu} \in C_{0}(\Omega)$.

In fact, the above theorem can be generalized to all finite sums of Toeplitz operators with bounded symbols and other equivalent conditions can be given. In our proof, the conditions on the weights $\nu$ and $\nu_{0}$ in Theorem B is owned to an application of Theorem 5.4.2.

The key of proving Theorem $\mathrm{B}$ is the following:

Theorem 5.4.3. Let $\Omega \subset \mathbb{C}^{d}$ be an irreducible bounded symmetric domain and suppose that

$$
\nu, \nu_{0}>\max \left\{d, p-1+\frac{r-1}{4} a+\sqrt{\left(\frac{r-1}{4} a\right)^{2}+\frac{r-1}{2} a\left(\frac{r-1}{2} a+p-1\right)}\right\} .
$$

Then for any $g \in L^{\infty}(\Omega)$ the following are equivalent: 
(1) $\widetilde{g}_{\nu_{0}} \in C_{0}(\Omega)$.

(2) $\widetilde{g}_{\nu} \in C_{0}(\Omega)$, for all $\nu$ such that $\left|\nu-\nu_{0}\right|>\frac{r-1}{2} a$.

(3) $T_{g}^{\nu_{0}}$ is compact on $H_{\nu_{0}}^{2}$.

(4) $T_{g}^{\nu}$ is compact on $H_{\nu}^{2}$, for all $\nu$ such that $\left|\nu-\nu_{0}\right|>\frac{r-1}{2} a$.

(5) $\widetilde{g}_{\nu} \in C_{0}(\Omega)$, for some $\nu$ such that $\left|\nu-\nu_{0}\right|>\frac{r-1}{2} a$.

(6) $T_{g}^{\nu}$ is compact on $H_{\nu}^{2}$, for some $\nu$ such that $\left|\nu-\nu_{0}\right|>\frac{r-1}{2} a$.

Proof. (i) We prove the implication $(6 \Longrightarrow 1)$ : Suppose that $T_{g}^{\nu}$ is compact for some $\nu$ such that $\left|\nu-\nu_{0}\right|>\frac{r-1}{2} a$. Then by Theorem A there exists $C\left(\nu, \nu_{0}\right)>0$ independent of $g$ such that:

$$
\left\|\widetilde{g}_{\nu_{0}}\right\|_{\infty} \leq C\left(\nu, \nu_{0}\right)\left\|T_{g}^{\nu}\right\|
$$

Since $T_{g}^{\nu}$ is compact, Theorem 5.4.1 tells us that there is a sequence $\left(g_{k}\right)_{k \in \mathbb{N}}$ of continuous functions with compact support such that:

$$
\left\|T_{g_{k}}^{\nu}-T_{g}^{\nu}\right\| \stackrel{k \rightarrow \infty}{\longrightarrow} 0
$$

which shows that

$$
\left\|\widetilde{g}_{\nu_{0}}-\widetilde{\left(g_{k}\right)}\right\|_{\nu_{0}} \|_{\infty} \stackrel{k \rightarrow \infty}{\longrightarrow} 0 .
$$

But each $\widetilde{\left(g_{k}\right)} \nu_{\nu_{0}} \in C_{0}(\Omega)$, hence $\widetilde{g}_{\nu_{0}} \in C_{0}(\Omega)$.

(ii) By the same argument it is easy to show the implication $(3 \Longrightarrow 2)$ using the inequality

$$
\left\|\tilde{g}_{\nu}\right\|_{\infty} \leq C\left(\nu_{0}, \nu\right)\left\|T_{g}^{\nu_{0}}\right\|_{\nu}
$$

Finally, we remark that by Theorem 5.4.2 we have ( $\Longleftrightarrow 3),(2 \Longleftrightarrow 4)$, and ( $5 \Longleftrightarrow 6)$. The implications $(4 \Longrightarrow 6)$ and $(2 \Longrightarrow 5)$ are trivial and we obtain the proof.

Proof of Theorem B: Let $\left(\nu, \nu_{0}\right)$ be two weights satisfying (5.4.2) and suppose that $T_{g}^{\nu_{0}}$ is compact. Then by the previous theorem it is sufficient to prove that $T_{g}^{\nu}$ is compact for $\left|\nu-\nu_{0}\right| \leq$ $\frac{r-1}{2} a$. Set $\gamma:=\max \left\{\nu_{0}, \nu\right\}$ and let $\nu_{1}=\gamma+1+\frac{r-1}{2} a$. Then we have

$$
\min \left\{\nu_{1}-\nu_{0}, \nu_{1}-\nu\right\}>\frac{r-1}{2} a \Longrightarrow T_{g}^{\nu_{1}} \text { is compact } \Longrightarrow T_{g}^{\nu} \text { is compact. }
$$

Remark 5.4.1. In [15], the authors proved that for a measurable function $g$ defined on the unit ball $\mathbb{B}^{n} \subset \mathbb{C}^{n}$ and having a bounded mean oscillation the compactness of the Toeplitz operator $T_{g}^{\lambda}$ on $A_{\lambda}^{2}\left(\mathbb{B}^{n}\right)$ (c.f. Section 1.4) is uniform w.r.t. weight $\lambda$ whenever $\lambda>-1$. We should remark here that our result is a generalization to that in [15] under the assumption of boundedness since we cover the case of all bounded symmetric domains. Indeed, for the case where $\Omega=\mathbb{B}^{n} \subset \mathbb{C}^{n}$ we know that $r=1, b=n-1$ and $p=n+1$. Substituting these quantities in Theorem $B$ we conclude that for any $g \in L^{\infty}\left(\mathbb{B}^{n}\right)$ the Toeplitz operator $T_{g}^{\lambda}$ is compact on $A_{\lambda}^{2}\left(\mathbb{B}^{n}\right)$ for all $\lambda>-1$ if and only if there is $\lambda_{0}>-1$ such that $T_{g}^{\lambda_{0}}$ is compact on $A_{\lambda_{0}}^{2}\left(\mathbb{B}^{n}\right)$. 
Finally, we would like to collect some open problems which are motivated by our results.

Question 1: Is the obstruction $\left|\nu-\nu_{0}\right|>\frac{r-1}{2} a$ in Theorem A necessary to obtain the inequality (5.1.1)?

Question 2: As mention in the above remark, it was proved in [15] that for the case of the unit ball $\Omega=\mathbb{B}^{n} \subset \mathbb{C}^{n}$ Theorem B holds for symbols $g \in B M O^{1}\left(\mathbb{B}^{n}\right)$ of bounded mean oscillation. This class of symbols strictly contains the bounded measurable functions. So one may ask if it is possible to extend Theorem $\mathrm{B}$ to the case of $B M O^{1}(\Omega)$ symbols for an arbitrary bounded symmetric domain $\Omega \subset \mathbb{C}^{n}$.

Question 3: Let $S_{p}\left(H_{\nu}^{2}\right)$ denote the Schatten-p-class over $H_{\nu}^{2}$ and assume that:

$$
\nu, \nu_{0}>\max \left\{d, p-1+\frac{r-1}{4} a+\sqrt{\left(\frac{r-1}{4} a\right)^{2}+\frac{r-1}{2} a\left(\frac{r-1}{2} a+p-1\right)}\right\} .
$$

For every $g \in L^{\infty}(\Omega)$, and all $1 \leq p<\infty$, is it true that:

$$
T_{g}^{\nu_{0}} \in S_{p}\left(H_{\nu_{0}}^{2}\right) \text { if and only if } T_{g}^{\nu} \in S_{p}\left(H_{\nu}^{2}\right) ?
$$




\section{Appendix A}

\section{A.1}

Density theorem This section is devoted to a detailed proof of the density criteria on compact operators acting on the Bergman space $H_{w}^{2}(\Omega)$ over any open domain $\Omega \subset \mathbb{C}^{n}$ (Theorem 1.2.2 ). This theorem was established by C. Berger and L. Coburn in the case of the Segal-Bargmann space with the standard weight $d \mu_{\frac{1}{2}}$ [35]. However using purely functional analytic methods we generalize this fact to any weighted Bergman space over $\Omega$. We use the same notation as in Chapter 1 .

For $X \in \mathcal{L}\left(H_{w}^{2}(\Omega)\right)$ we define the function $K^{X}(z, w)=\left(X^{\star} K(\cdot, w)\right)(z)$ on $\Omega \times \Omega$. It is easy to check that the two variable function $K^{X}$ satisfies:

(a) $K^{X}(z, w)=\overline{(X K(\cdot, z))(w)}$.

(b) $K^{X}$ is holomorphic in $z$ and anti-holomorphic in $w$.

Since span $\left\{K_{z}\right\}_{z \in \Omega}$ is dense in $H_{w}^{2}$ and $X$ is bounded, by (b) it follows that if $K^{X}(z, z)=0$ for all $z \in \Omega$ then $X \equiv 0$. Moreover, if $X$ is of trace class we have the following relation between its trace and $K^{X}$.

Proposition A.1.1. If $X \in$ trace class $\left(H_{w}^{2}(\Omega)\right)$, then the trace of $X$ is given by:

$$
\operatorname{tr}(X)=\int_{\Omega} \overline{K^{X}(a, a)} d \mu(a)
$$

Proof. Let $\left\{e_{n}\right\}_{n \in \mathbb{N}}$ be an orthonormal basis of $H_{w}^{2}(\Omega)$, then for any $a \in \Omega$ we know that ( c.f. Theorem 1.2.1) $K(\cdot, a)=\sum_{n \in \mathbb{N}} e_{n}(\cdot) \overline{e_{n}(a)}$. Therefore, by the continuity of $X$ we have

$$
X(K(\cdot, a))(z)=X\left(\sum_{n \in \mathbb{N}} e_{n} \overline{e_{n}(a)}\right)(z)=\sum_{n \in \mathbb{N}} X\left(e_{n}\right)(z) \overline{e_{n}(a)}=\langle X K(\cdot, a), K(\cdot, z)\rangle .
$$

Integrating both sides of the above equation over $\Omega$ we obtain

$$
\begin{aligned}
\int_{\Omega} X(K(\cdot, a))(a) d \mu(a) & =\int_{\Omega} \overline{K^{X}(a, a)} d \mu(a)=\int_{\Omega}\langle X K(\cdot, a), K(\cdot, a)\rangle d \mu(a) \\
& =\sum_{n \in \mathbb{N}} \int_{\Omega} X\left(e_{n}\right)(a) \overline{e_{n}(a)} d \mu(a)=\sum_{n \in \mathbb{N}}\left\langle X\left(e_{n}\right), e_{n}\right\rangle=\operatorname{tr}(X) .
\end{aligned}
$$


Proposition A.1.2. Let $X, Y \in \mathcal{L}\left(H_{w}^{2}(\Omega)\right)$, such that $Y X \in$ trace class $\left(H_{w}^{2}(\Omega)\right)$, then :

$$
\operatorname{tr}(Y X)=\int_{\Omega} d \mu(a) \int_{\Omega} \overline{K^{Y}(z, a) K^{X}(a, z)} d \mu(z) .
$$

Proof. Note that, for all $z, w \in \Omega$, we have:

$$
\begin{aligned}
\overline{K^{Y X}(z, w)} & =Y X(K(\cdot, z))(w)=\langle Y X(K(\cdot, z)), K(\cdot, w)\rangle=\left\langle X(K(\cdot, z)), Y^{*} K(\cdot, w)\right\rangle \\
& =\int_{\Omega} X(K(\cdot, z))(a) \overline{Y^{*} K(\cdot, w)(a)} d \mu(a)=\int_{\Omega} \overline{K_{v}^{X}(z, a) K_{v}^{Y}(a, w)} d \mu_{v}(a) .
\end{aligned}
$$

Now by Proposition A.1.1, we have:

$$
\operatorname{tr}(Y X)=\int_{\Omega} \overline{K^{Y X}(a, a)} d \mu(a)=\int_{\Omega} \int_{\Omega} \overline{K^{X}(a, z) K^{Y}(z, a)} d \mu(z) d \mu(a) .
$$

Remark A.1.1. Suppose $g \in \tau_{w}(\Omega):=\left\{f: \Omega \longrightarrow \mathbb{C}^{n} \mid f K_{z} \in L^{2}\left(\Omega, d \mu_{w}\right)\right.$, for all $\left.z \in \Omega\right\}$ and $T_{g} \in \mathcal{L}\left(H_{w}^{2}(\Omega)\right)$ then:

$$
\bar{g} K(\cdot, a)-K^{T_{g}}(\cdot, a):=p(\cdot, a) \in\left(H_{w}^{2}(\Omega)\right)^{\perp} \quad \forall a \in \Omega,
$$

where $\left(H_{w}^{2}(\Omega)\right)^{\perp}:=\left\{f \in L_{w}^{2} \mid\langle f, g\rangle_{w}=0, \forall g \in H_{w}^{2}(\Omega)\right\}$.

Proof.

$$
\begin{aligned}
K^{T_{g}}(z, a)=\overline{T_{g}(K(\cdot, z))(a)} & =\int_{\Omega} \overline{g(u)} K(z, u) K(u, a) d \mu_{w}(u) \\
& =\int_{\Omega} \overline{g(u)} K(u, a) \overline{K(u, z)} d \mu_{w}(u) \\
& =\langle\overline{g(\cdot)} K(\cdot, a), K(\cdot, z)\rangle=P(\bar{g} K(\cdot, a))(z),
\end{aligned}
$$

which shows that $\bar{g} K(\cdot, a)-K^{T_{g}}(\cdot, a) \in\left(H_{w}^{2}(\Omega)\right)^{\perp} \forall a \in \Omega$.

The following proposition is a generalization of Proposition 5.3.1 in Chapter 5.

Proposition A.1.3. Let $g \in \tau_{w}(\Omega), X \in \mathcal{L}\left(H_{w}^{2}(\Omega)\right)$ and suppose the following conditions hold:

1. $T_{g} \in \mathcal{L}\left(H_{w}^{2}(\Omega)\right)$.

2. $T_{g} X \in$ trace class $\left(H_{w}^{2}(\Omega)\right)$.

3. $\int_{\Omega} \int_{\Omega}|g(z)||K(u, z)|\left|K^{X}(u, z)\right| d \mu(z) d \mu(u)<\infty$.

then

$$
\operatorname{tr}\left(T_{g} X\right)=\int_{\Omega} g(z) \overline{K^{X}(z, z)} d \mu(z)
$$


Proof. By proposition A.1.2 and the above remark we get

$$
\begin{aligned}
\operatorname{tr}\left(T_{g} X\right) & =\int_{\Omega} \int_{\Omega} \overline{K^{X}(a, z) K^{T_{g}}(z, a)} d \mu(z) d \mu(a) \\
& =\int_{\Omega} \int_{\Omega} \overline{K^{X}(a, z)}(\overline{p(\cdot, a)}+g(z) \overline{K(z, a)}) d \mu(z) d \mu(a) \\
& =\int_{\Omega} g(z) \int_{\Omega} \overline{K^{X}(a, z)} K(a, z) d \mu(a) d \mu(z)=\int_{\Omega} g(z)\left\langle K(\cdot, z), K^{X}(\cdot, z)\right\rangle d \mu(z) \\
& =\int_{\Omega} g(z) \overline{\left\langle K^{X}(\cdot, z), K(\cdot, z)\right\rangle} d \mu(z)=\int_{\Omega} g(z) \overline{P\left(K^{X}(\cdot, z)\right)(z)} d \mu(z) \\
& =\int_{\Omega} g(z) \overline{K^{X}(z, z)} d \mu(z) .
\end{aligned}
$$

The following proposition is the key to prove the density criteria:

Proposition A.1.4. For $g$ bounded, measurable function of compact support $S_{g} \subset \Omega$. $T_{g}$ is of trace class on $H_{w}^{2}$ and for $X \in \mathcal{L}\left(H_{w}^{2}\right)$ we have:

$$
\operatorname{tr}\left(T_{g} X\right)=\int_{\Omega} g(u) \overline{K^{X}(u, u)} d \mu_{w}(u) .
$$

Proof. The fact that $T_{g}$ is of trace class is proved in Section 1.2 of Chapter 1. In order to obtain (A.1.2) we have only to check condition 3 of Proposition A.1.3:

$$
\begin{aligned}
& \int_{\Omega} \int_{\Omega}|g(z)||K(u, z)|\left|K^{X}(u, z)\right| d \mu_{w}(u) d \mu_{w}(z) \\
& =\int_{\Omega}|g(z)| d \mu(z) \int_{\Omega}|K(u, z)| \mid X^{*}\left(K(\cdot, z)(u) \mid d \mu_{v}(u)\right. \\
& \leq \int_{\Omega}|g(z)|\left\|X^{*}\right\|\|K(\cdot, z)\|^{2} d \mu(z) \\
& =\left\|X^{*}\right\| \int_{S_{g}}|g(z)| K(z, z) d \mu(z) \leq\left\|X^{*}\right\|\left\|g_{\infty}\right\| \int_{S_{g}} K(z, z) d \mu(z)<\infty .
\end{aligned}
$$

Proof of Theorem 1.2.2: Denote by $\mathcal{H}(\Omega)$ the space of all compact operators acting on $H_{w}^{2}(\Omega)$. Then the dual space $\mathcal{H}^{*}=S_{1}$ is the space of all trace class operator on $H_{w}^{2}(\Omega)$ and the duality is given by (c.f. Chapter 1 in [186])

$$
\langle Y, X\rangle_{\mathcal{H}}=\operatorname{tr}(Y X), \quad \forall Y \in S_{1}, X \in \mathcal{H} .
$$

Suppose $C^{\|\cdot\|} \neq \mathcal{H}$, then by the Hahn-Banach Theorem $\exists X \neq 0 X \in S_{1}$ and $X_{\mid C}=0$. Then by the above proposition we have

$$
\operatorname{tr}\left(T_{g} X\right)=\int_{\Omega} g(u) \overline{K^{X}(u, u)} d \mu_{w}(u)=0
$$

for every continuous function with $g$ with compact support, hence $K^{X}(w, w)=0$ which is possible if and only if $X=0$. 


\section{A.2}

\section{A.2.1 Proof of Remark 2.4.2}

Theorem A.2.1. Let

$$
f(z)=f_{m, \delta}(z)=z^{2} \bar{z}=r^{3} e^{i \theta}, \text { and } g(z)=\psi_{2}(r) e^{i \theta}\left(r^{6}-2 r^{4}\right) e^{2 i \theta}
$$

be the functions defined in Remark 2.4.2. Then the Toeplitz operators $\mathcal{T}_{f}^{s}$ and $\mathcal{T}_{g}^{s}$ commute on $\mathbb{P}[z]$ if and only if $s=1$.

Proof. We apply the Toeplitz operators $T_{f}$ and $T_{g}$ acting on $H^{2}$ to the monomials $\left\{z^{n}\right\}_{n \in \mathbb{N}_{0}}$. Using Proposition 2.2.2 we obtain

1.

$$
\begin{aligned}
T_{g}\left(z^{n}\right)=P\left(\Psi r^{n} e^{i n \theta}\right) & =P\left(r^{6+n} e^{i(n+2) \theta}-2 r^{n+4} e^{i(n+2) \theta}\right) \\
& =\left\{2 M\left[e^{-r^{2}}\right](2 n+10)-4 M\left[e^{-r^{2}}\right](2 n+8)\right\} \frac{z^{n+2}}{(n+2) !} \\
& =\{2 \Gamma(n+5)-4 \Gamma(n+4)\} \frac{z^{n+2}}{(n+2) !} .
\end{aligned}
$$

2. $T_{f} z^{n}=T_{r^{3} e^{i \theta}} z^{n}=P\left(r^{n+3} e^{i(n+1) t h e t a}(z)=2 M\left[e^{-r^{2}}\right](2 n+6) \frac{z^{n+1}}{(n+1) !}=2 \Gamma(n+3) \frac{z^{n+1}}{(n+1) !}\right.$.

Combining the above equalities we get

1. $T_{g} T_{f} z^{n}=2 \frac{\Gamma(n+3)}{(n+1) !}\{2 \Gamma(n+6)-4 \Gamma(n+5)\} \frac{z^{n+3}}{(n+3) !}$.

2. $T_{f} T_{g} z^{n}=\frac{2 \Gamma(n+5)-4 \Gamma(n+4)}{(n+2) !} 2 \Gamma(n+5) \frac{z^{n+3}}{(n+3) !}$.

Moreover, using Equation (2.4.20) we apply the Toeplitz operator $\mathcal{T}_{f}^{s}$ and $\mathcal{T}_{g}^{s}$ acting on $\mathcal{H}_{s}^{2}$ to the monomials $\left\{z^{n}\right\}_{n \in \mathbb{N}_{0}}$ and we obtain

1. $\mathcal{T}_{f}^{s} z^{n}(z)=T_{f\left(\frac{\dot{\sqrt{s}}}{)}\right)} \sqrt{s} z^{n}(\sqrt{s} z)=s^{-\frac{3+n}{2}} T_{f} z^{n}(\sqrt{s} z)=s^{-\frac{3}{2}} 2 \Gamma(n+3) \frac{z^{n+1}}{(n+1) !}$.

2. $\mathcal{T}_{g}^{s} z^{n}(z)=\frac{1}{s^{\frac{n}{2}}} T_{\Psi\left(\frac{\dot{\sqrt{s}}}{)}\right.} z^{n}(\sqrt{s} z)=\frac{1}{s^{\frac{n}{2}}}\left\{\frac{2 \Gamma(n+5)}{s^{3}}-\frac{4 \Gamma(n+4)}{s^{2}}\right\} s^{\frac{n}{2}} \cdot s \frac{z^{n+2}}{(n+2) !}=\left\{\frac{2 \Gamma(n+5)}{s^{3}}-\right.$ $\left.\frac{4 \Gamma(n+4)}{s^{2}}\right\} s \frac{z^{n+2}}{(n+2) !}$.

Therefore

$$
\mathcal{T}_{f}^{s} \mathcal{T}_{g}^{s} z^{n}(z)=\left\{\frac{2 \Gamma(n+5)}{s^{3}}-\frac{4 \Gamma(n+4)}{s^{2}}\right\} \frac{s}{(n+2) !} s^{-\frac{3}{2}} 2 \Gamma(n+5) \frac{z^{n+3}}{(n+3) !}
$$

and

$$
\mathcal{T}_{g}^{s} \mathcal{T}_{f}^{s} z^{n}(z)=s^{-\frac{3}{2}} 2 \Gamma(n+3) \frac{s}{(n+1) !}\left\{\frac{2 \Gamma(n+6)}{s^{3}}-\frac{4 \Gamma(n+5)}{s^{2}}\right\} \frac{z^{n+3}}{(n+3) !}
$$

Hence $T_{f}^{s} T_{\Psi}^{s}=T_{\Psi}^{s} T_{f}^{s}$ if and only if for all $n \in \mathbb{N}_{0}$ the following equation holds

$$
\left\{\frac{2 \Gamma(n+5)}{s^{3}}-\frac{4 \Gamma(n+4)}{s^{2}}\right\} \frac{1}{(n+2) !} \Gamma(n+5)=\Gamma(n+3) \frac{1}{(n+1) !}\left\{\frac{2 \Gamma(n+6)}{s^{3}}-\frac{4 \Gamma(n+5)}{s^{2}}\right\} .
$$

By choosing $n=0$ the above equation holds true only in the case $s=1$. 


\section{A.2.2 Proof of Proposition 2.5.1}

Let $\Psi \in S$ and suppose that $T_{f_{m, \delta}} T_{\Psi}=T_{\Psi} T_{f_{m, \delta}}$. Then for each $j<0$ the following functional equations:

$$
M\left[\Psi_{j} e^{-r^{2}}\right](2 z+2 \delta+j+2)=M\left[\Psi_{j} e^{-r^{2}}\right](2 z+j+2) \prod_{l=1}^{\delta-j}(z+j+l) \prod_{l=1}^{-j}\left(z+j+\frac{\delta+m}{2}+l\right)^{-1}
$$

hold in the half plane $\operatorname{Re}(z)>-j-1$.

Proof. Fix $j<0$, by Equation (2.3.1) and under the assumption $T_{f_{m, \delta}} T_{\Psi}=T_{\Psi} T_{f_{m, \delta}}$ we know that for every $k \in \mathbb{N}_{0}$ such that $k \geqslant-j$ :

$$
M\left[\Psi_{j} e^{-r^{2}}\right](2 k+2 \delta+j+2) \frac{\Gamma(k+j+1)}{\Gamma(k+\delta+1)}=\frac{\Gamma\left(k+j+1+\frac{\delta+m}{2}\right)}{\Gamma\left(k+1+\frac{\delta+m}{2}\right)} M\left[\Psi_{j} e^{-r^{2}}\right](2 k+j+2) .
$$

Using the functional equation of the Gamma function the above equation can written in the following form

$$
M\left[\Psi_{j} e^{-r^{2}}\right](2 k+2 \delta+j+2)=M\left[\Psi_{j} e^{-r^{2}}\right](2 k+j+2) \prod_{l=1}^{\delta-j}(k+j+l) \prod_{l=1}^{-j}\left(k+j+\frac{\delta+m}{2}+l\right)^{-1} .
$$

According to (2.2.2) we know that for $\operatorname{Re}(z)>-j-1$

$$
\begin{gathered}
\prod_{l=1}^{\delta-j}(z+j+l)^{-1}=2 M\left[r^{2 j} Q_{\delta-j}\left(r^{2}\right)\right](2 z), \text { and } \\
\prod_{l=1}^{-j}\left(z+j+\frac{\delta+m}{2}+l\right)^{-1}=2 M\left[r^{2 j+\delta+m} Q_{-j}\left(r^{2}\right)\right](2 z) .
\end{gathered}
$$

Substituting these quantities into (A.2.2), we conclude that

$$
\begin{aligned}
& M\left[r^{j+2 \delta+2} \Psi_{j}(r) e^{-r^{2}}\right](2 k) \cdot M\left[r^{2 j} Q_{\delta-j}\left(r^{2}\right)\right](2 k) \\
& \quad=M\left[r^{j+2} \Psi_{j} e^{-r^{2}}\right](2 k) \cdot M\left[r^{2 j+\delta+m} Q_{-j}\left(r^{2}\right)\right](2 k),
\end{aligned}
$$

for all integers integers $k$ such that $k \geqslant-j$. Now consider

$$
v_{j}(r):=r^{2 j} Q_{\delta-j}\left(r^{2}\right) e^{r^{2}}, \text { and } u_{j}(r):=r^{j+2 \delta+2} \Psi_{j}(r) .
$$

Since $Q_{\delta-j}$ is supported in $[0,1]$ and $\Psi_{j} \in \mathcal{A}$, then $u_{j}, v_{j} \in \mathcal{A}$. Therefore, by Lemma 2.3.1 the convolution product $f_{u_{j}} * f_{v_{j}}(r)$ exists for all $r \geqslant 0$, and there is $h_{j} \in \mathcal{A}$ such that:

$$
\left[r^{2 j} Q_{\delta-j}\left(r^{2}\right) * r^{j+2 \delta+2} \Psi_{j}(r) e^{-r^{2}}\right](r)=h_{j}(r) e^{-r} .
$$

The same technique can be used on the right hand side of (A.2.3) to show that there is $n_{j} \in \mathcal{A}$ such that

$$
\left[r^{j+2} \Psi_{j} e^{-r^{2}} * r^{2 j+\delta+m} Q_{-j}\left(r^{2}\right)\right](r)=n_{j}(r) e^{-r}
$$


By substituting these relations into (A.2.3) and applying the convolution theorem for the Mellin transform, we obtain for any $k \in \mathbb{N}_{0}$ such that $k \geqslant-j$ :

$$
M\left[\left(h_{j}-n_{j}\right) e^{-r}\right](2 k)=M\left[\frac{\left(h_{j}-n_{j}\right)}{r} e^{-r}\right](2 k+1)=0 .
$$

By Lemma 2.3.2 we have $h_{j}=n_{j}$ a.e. on $\mathbb{R}_{+}$, i.e the equality (A.2.1) holds on the half plane $\operatorname{Re}(z)>-j-1$.

\section{A.2.3 Proof of Proposition 2.5.2}

Let $\Psi \in S$ and suppose that $T_{f_{m, \delta}} T_{\Psi}=T_{\Psi} T_{f_{m, \delta}}$. Then for each $j<0$ there exists a trigonometric polynomial $\sum_{|l|<\frac{\delta}{4}} a_{l} e^{\frac{2 \pi i l z}{\delta}}$ such that for $\operatorname{Re}(z)>-j-1$ we have:

$$
M\left[\Psi_{j} e^{-r^{2}}\right](2 z+j+2)=H_{j}(z) \sum_{|l|<\frac{\delta}{4}} a_{l} e^{\frac{2 \pi i l z}{\delta}} .
$$

Proof. Using the calculations done in Proposition 2.4.3, we conclude that

$$
\begin{aligned}
& \prod_{l=1}^{\delta-j} \Gamma\left(\frac{z+j+l}{\delta}\right) \\
& \quad=\exp \left\{\sum_{l=1}^{\delta-j}\left(\frac{z+j+l}{\delta}-\frac{1}{2}\right) \log \left(\frac{z}{\delta}\right)+O(1)-\left(\frac{z+j+l}{\delta}\right)+\frac{\log (2 \pi)}{2}\right\} \times\left\{1+o\left(\frac{1}{z}\right)\right\} \\
& \quad=\exp \left\{\left[\frac{z}{\delta} \log \left(\frac{z}{\delta}\right)-\frac{z}{\delta}\right](\delta-j)+(\delta-j) \frac{(j+1)}{2 \delta} \log \left(\frac{z}{\delta}\right)+O(1)\right\} \times\left\{1+o\left(\frac{1}{z}\right)\right\}
\end{aligned}
$$

as $|z| \longrightarrow \infty$. Similarly, we have

$$
\begin{aligned}
& \prod_{l=1}^{-j}\left[\Gamma\left(\frac{z+j+\frac{\delta+m}{2}+l}{\delta}\right)\right]^{-1} \\
& =\exp \left\{-\sum_{l=1}^{-j}\left(\frac{z+j+\frac{\delta+m}{2}+l}{\delta}+\frac{1}{2}\right) \log \left(\frac{z}{\delta}\right)+O(1)+\left(\frac{z+j+\frac{\delta+m}{2}+l}{\delta}\right)\right\} \times\left\{1+o\left(\frac{1}{z}\right)\right\} \\
& =\exp -\left\{\left[\frac{z}{\delta} \log \left(\frac{z}{\delta}\right)-\frac{z}{\delta}\right](-j)+(-j) \frac{j+m+1}{2 \delta} \log \left(\frac{z}{\delta}\right)+O(1)\right\} \times\left\{1+o\left(\frac{1}{z}\right)\right\} \\
& \text { as }|z| \longrightarrow \infty
\end{aligned}
$$

Therefore

$$
\begin{aligned}
& \prod_{l=1}^{\delta-j} \Gamma\left(\frac{z+j+l}{\delta}\right) \prod_{l=1}^{-j}\left[\Gamma\left(\frac{z+j+\frac{\delta+m}{2}+l}{\delta}\right)\right]^{-1}= \\
& \exp \left\{z \log \left(\frac{z}{\delta}\right)+s \log \left(\frac{z}{\delta}\right)-z+O(1)\right\} \times\left\{1+o\left(\frac{1}{z}\right)\right\}
\end{aligned}
$$


where $s:=\frac{j(m-1)+1}{2 \delta}+j$. According to (2.4.8) together the above asymptotic behavior of $H_{j}$ we know that the function $\tau_{j}(z)=\frac{M\left[\Psi_{j} e^{-r^{2}}\right](2 z+j+2)}{H_{j}(z)}$ satisfies

$$
\left|\tau_{j}(z)\right| \leqslant \frac{2 c \Gamma\left(\sigma+\frac{j}{2}+\frac{c}{2}+1\right)}{\exp \{(\sigma+s) \log (|z|)-\sigma-t \cdot \arg (z)+O(1)\} \times\left\{1+O\left(\frac{1}{|z|}\right)\right\}},
$$

where $z=\sigma+i t, \sigma>0$ is sufficiently large and $c$ is independent of $z$. However, Equation (A.2.4) shows that $\tau_{j}$ is $\delta$-periodic hence it can be extended to entire function on the complex plane. Therefore, for studying the growth of $\tau_{j}$ as $|z| \longrightarrow \infty$, we can suppose that in the above estimate $\sigma$ is large and varying in an interval of length $\delta$. According to (A.2.5), there is a constant $C>0$ such that:

$$
\left|\tau_{j}(z)\right| \leqslant \frac{C}{1+t^{2}} e^{t \arg (z)}<\frac{C}{1+t^{2}} e^{\frac{\pi}{2}|z|} .
$$

Hence Lemma 2.4.1 shows that there is a trigonometric polynomial $\sum_{|l|<\frac{\delta}{4}} a_{l} e^{\frac{2 \pi i l z}{\delta}}$ satisfying (A.2.4).

\section{A.3}

\section{A.3.1 Proof of Proposition 4.2.3}

For the proof we need the next Lemma which easily follows from Theorem 3 of Appendix A in [84].

Lemma A.3.1. [84]

Let $A$ and $D$ be two Hermitian $n \times n$ complex matrices such that

$$
\|A\| \leq \frac{1}{2}, \quad\|D\| \leq \frac{1}{2}, \quad \text { and } \quad\|A D\|<\frac{1}{4} .
$$

Then for any $u, v \in \mathbb{C}^{n}$ we have:

$$
\begin{aligned}
& \int_{\mathbb{C}^{n}} \exp \{z A z+\bar{z} D \bar{z}+u z+v \bar{z}\} d \mu(z) \\
& =\frac{1}{\sqrt{\operatorname{det}(I d-4 A D)}} \exp \left\{u D(I d-4 A D)^{-1} u+v(I d-4 A D)^{-1} u+v A(I d-4 A D)^{-1} v\right\}
\end{aligned}
$$

For a complex matrix $A$ we denote by $\bar{A}, A^{T}$ the complex conjugate and the transpose of the matrix $A$, respectively. Moreover, we write $A^{\star}:=\bar{A}^{T}$ for the conjugate transpose of $A$. We are able now to give a complete proof of Proposition 4.2.3. 
Proof. We write $u=\left(u_{1}, \cdots, u_{n}\right) \in \mathbb{C}^{n}$ and denote by $\frac{\partial}{\partial z}$ the $n$-column matrix with differential operator entries $\frac{\partial}{\partial z_{j}}$ i.e. for any entire function $g$ on $\mathbb{C}^{n}$ we have

$$
\frac{\partial g}{\partial z}=\left(\frac{\partial g}{\partial z_{1}} \cdots \frac{\partial g}{\partial z_{n}}\right)^{T}
$$

Let $E=\left(e_{k j}\right) \in M_{n}(\mathbb{C})$ be Hermitian s.t. $\|E\|<\frac{1}{2}$ and the matrix $(I d-F)(I d+F)^{-1}$ is positive definite where $F:=E+\bar{E}$. In order to obtain (4.2.16) we apply $\frac{\partial}{\partial z}$ to the complex valued function $e^{u \cdot z+z E z}$

$$
\begin{aligned}
& \frac{\partial}{\partial z} e^{u \cdot z+z E z}=\frac{\partial}{\partial z} \exp \left\{\sum_{l} u_{l} z_{l}+\sum_{k, j} z_{k} e_{k j} z_{j}\right\} \\
& =e^{u \cdot z+z E z}\left(\left(u_{1}+\sum_{j \neq 1} e_{1 j} z_{j}+\sum_{k \neq 1} z_{k} e_{k 1}+2 z_{1} e_{11}\right), \cdots\right. \\
& \left.\quad\left(u_{n}+\sum_{j \neq n} e_{n j} z_{j}+\sum_{k \neq n} z_{k} e_{k n}+2 z_{n} e_{n n}\right)\right)^{T} \\
& =(F z+u) e^{u \cdot z+z E z}
\end{aligned}
$$

Therefore, $e^{u \cdot z+z E z}$ is in the kernel of the following column matrix with differential operator entries acting on $H^{2}\left(\mathbb{C}^{n}\right)$ solution of the differential operator

$$
L:=\frac{\partial}{\partial z}-F z-u
$$

Hence $\beta_{t}^{-1} \exp \{u \cdot z+z E z\}$ is in the kernel of the corresponding operator $\chi:=\beta_{t}^{-1} \Delta \beta_{t}$ on $L^{2}\left(\mathbb{R}^{n}\right)$. By (4.2.12) and (4.2.13) we have:

$$
\begin{aligned}
\chi & =\frac{t}{2} x+\frac{1}{t} \frac{\partial}{\partial x}-F\left(\frac{t}{2} x-\frac{1}{t} \frac{\partial}{\partial x}\right) \\
& =\frac{t}{2}(I d-F) x+\frac{1}{t}(I d+F) \frac{\partial}{\partial x}-u .
\end{aligned}
$$

In order to find the general solution of the ,,column matrix differential operator" (A.3.2) we replace $\chi$ by

$$
\begin{aligned}
\chi_{1} & :=(I d+F)^{-1} \chi \\
& =\frac{t}{2}(I d+F)^{-1}(I d-F) x+\frac{1}{t} \frac{\partial}{\partial x}-(I d+F)^{-1} u .
\end{aligned}
$$

Now let $f \in L^{2}\left(\mathbb{R}^{n}\right)$ be such that $\chi_{1} f=0$. Solving the above system of first order differential equation leads to the existence of a constant $c$ such that:

$$
f(x)=c \exp \left\{u(I d+F)^{-1} t x-\frac{t^{2}}{4} x(I d-F)(I d+F)^{-1} x\right\} .
$$

Since the Bargmann transform is an isometry we deduce the constant $c$ by calculating the norm of $f$ and the norm of $e^{u z+z E z}$ in the corresponding spaces. Applying Equation (A.3.1) 
to $e^{u \cdot z+z E z}$ with $A=E, D=\bar{E}$ and $v=\bar{u}$ we obtain:

$$
\begin{aligned}
& \left\|e^{u \cdot z+z E z}\right\|^{2}=\int_{\mathbb{C}^{n}} \exp \{z E z+\bar{z} \bar{E} \bar{z}+u z+\overline{u z}\} d \mu(z) \\
& =\frac{1}{\sqrt{\operatorname{det}(I d-4 E \bar{E})}} \exp \left\{u \bar{E}(I d-4 E \bar{E})^{-1} u+\bar{u}(I d-4 E \bar{E})^{-1} u+\bar{u} E(I d-4 E \bar{E})^{-1} \bar{u}\right\}
\end{aligned}
$$

To calculate $\|f\|^{2}$ write Equation (A.3.3) in an another way. Put $K:=(I d-F)(I d+F)^{-1}$ and $\bar{z}:=\sqrt{K^{-1}}(I d+F)^{-1} u \in \mathbb{C}^{n}$. Using the change of variable $y=\sqrt{K} x$ it is easy to check that

$$
\begin{aligned}
f(x)=f\left(\sqrt{K^{-1}} y\right) & =c \exp \left\{u(I d+F)^{-1} t \sqrt{K^{-1}} y-\frac{t^{2}}{4} y^{2}\right\} \\
& =c t^{\frac{-n}{2}}(2 \pi)^{\frac{n}{4}} \Phi_{z}^{t}(y) e^{\frac{\bar{z}^{2}}{2}} \\
& =c t^{\frac{-n}{2}}(2 \pi)^{\frac{n}{4}} \Phi_{z}^{t}(\sqrt{K} x) \exp \left\{\frac{u(I d+F)^{-1} K^{-1}(I d+F)^{-1} u}{2}\right\} \\
& =c t^{\frac{-n}{2}}(2 \pi)^{\frac{n}{4}} \Phi_{z}^{t}(\sqrt{K} x) \exp \left\{\frac{u(I d+F)^{-1}(I d-F)^{-1} u}{2}\right\} .
\end{aligned}
$$

Applying equation (4.2.1) together with (A.3.5) we get:

$$
\begin{gathered}
\|f\|_{L^{2}\left(\mathbb{R}^{n}\right)}^{2}=c^{2} t^{-n}(2 \pi)^{\frac{n}{2}} \exp \left\{u(I d+F)^{-1}(I d-F)^{-1} u\right\}\left\|\Phi_{z}^{t}(\sqrt{K} x)\right\|^{2} \\
=c^{2} t^{-n}(2 \pi)^{\frac{n}{2}} \exp \left\{u(I d+F)^{-1}(I d-F)^{-1} u\right\} \frac{e^{|z|^{2}}}{\operatorname{det} \sqrt{K}} \\
=c^{2} \frac{t^{-n}(2 \pi)^{\frac{n}{2}}}{\operatorname{det} \sqrt{(I d-F)(I d+F)^{-1}}} \exp \left\{u(I d+F)^{-1}(I d-F)^{-1} u\right\} \\
\times \exp \left\{u(I d+F)^{-1}(I d-F)^{-1} \bar{u}\right\}
\end{gathered}
$$

Comparing (A.3.4) and (A.3.6) we obtain:

$$
\begin{aligned}
& c^{2}=t^{n}(2 \pi)^{-\frac{n}{2}} \sqrt{\frac{\operatorname{det}(I d-F)(I d+F)^{-1}}{\operatorname{det}(I d-4 E \bar{E})}} \exp \left\{u \bar{E}(I d-4 E \bar{E})^{-1} u+\bar{u}(I d-4 E \bar{E})^{-1} u\right. \\
& \left.+\bar{u} E(I d-4 E \bar{E})^{-1} \bar{u}-u(I d+F)^{-1}(I d-F)^{-1} u-u(I d+F)^{-1}(I d-F)^{-1} \bar{u}\right\} .
\end{aligned}
$$




\section{Bibliography}

[1] R. A. Adams, Sobolev Spaces, Academic Press, New York, 1975.

[2] P. Ahern, M. Flores and W. Rudin, An invariant volume-mean-value property, J. Funct. Anal. 111 (1993), pp. 380-397.

[3] P. Ahern and Z. C̆učković, A theorem of Brown-Halmos type for Bergman space Toeplitz operators, J. Funct. Anal. 187 (2001), pp. 200-210.

[4] P. Ahern and Z. Čučković, Products of Toeplitz operators on the Bergman space, Illinois J. Math. 45 (2001), pp. 113-121.

[5] J. Arazy and H. Upmeier, Invariant inner product in spaces of holomorphic functions on bounded symmetric domains, Doc. Math. 2 (1997), pp. 213-261.

[6] N. Aronszajn, Reproducing and pseudo-reproducing kernels and their application to the partial differential equations of physics, Studies in partial differential equations of physics, Harvard University, Graduate School of Engineering, Cambridge, Massachusetts, 1948.

[7] N. Aronszajn, Theory of reproducing kernels, Trans. Amer. Math. Soc. 68 (1950), pp. 337-404.

[8] S. Axler and Z. Čučković, Commuting Toeplitz operators with harmonic symbols, Integr. Equ. Oper. Th. 14 (1991), pp. 1-12.

[9] S. Axler, Z. Čučković and N. V. Rao, Commutants of analytic Toeplitz operators on the Bergman space, Proc. Amer. Math. Soc. 128 (2000), pp. 1951-1953.

[10] S. Axler and D. Zheng, Compact operators via the Berezin transform, Indiana Univ. Math. J. 47 (1998), pp. 387-400.

[11] S. Axler and D. Zheng, The Berezin transform on the Toeplitz algebra, Studia Math. 127 (1998), pp. 113-136.

[12] V. Bargmann, On a Hilbert space of analytic functions and an associated integral transform, Part I, Comm. Pure. Appl. Math. 14 (1961), pp. 187-214.

[13] V. Bargmann, On a Hilbert space of analytic functions and an associated integral transform, Part II, Comm. Pure. Appl. Math. 20 (1967), pp. 1-101. 
[14] W. Bauer, Berezin Toeplitz quantization and composition formulas, J. Funct. Anal. 256 (2009), pp. 3107-3142.

[15] W. Bauer, L. Coburn and J. Isralowitz, Heat flow, BMO, and the compactness of Toeplitz operators, J. Funct. Anal. 259 (2010), pp. 57-78.

[16] W. Bauer and T. Le, Algebraic properties and the finite rank problem for Toeplitz operators on the Segal-Bargmann space, J. Funct. Anal. 261 (2011), pp. 2617-2640.

[17] W. Bauer and Y. J. Lee, Commuting Toeplitz operators on the Segal-Bargmann space, J. Funct. Anal. 260 (2011), pp. 460-489.

[18] W. Bauer and K. Furutani, Compact operators and the pluriharmonic Berezin transform, Internat. J. Math. 19 (2008), pp. 645-669.

[19] W. Bauer, K. Furutani and C. Iwasaki, Spectral analysis and geometry of sub-Laplacians and Grushin type operators, Partial Differential Equation and Spectral Theory, pp. 183290, Oper. Theory Adv. Appl. 211, Birkhäuser, Basel, 2011.

[20] W. Bauer and J. Isralowitz, Compactness characterization of operators in the Toeplitz algebra of the Fock space $F_{\alpha}^{p}$, J. F. Anal. Doi: 10.1016/j.jfa.2012.04.020, (2012)

[21] W. Bauer and H. Issa, Commuting Toeplitz operators with quasi-homogeneous symbols on the Segal-Bargmann space, J. Math. Anal. Appl. 386 (2012), pp. 213-235.

[22] W. Bauer and N. Vasilevski, Banach algebras of commuting Toeplitz operators on the unit ball via the quasi-hyperbolic group, Operator Theory: Advances and Applications, Birkhäser, Vol. 218 pp. 155-175, 2012.

[23] W. Bauer and N. Vasilevski, Commutative Toeplitz Banach algebras on the ball and quasinilpotent group action, Integr. Equ. Oper. Th. 72 (2012), pp. 223-240.

[24] R. Beals, B. Gaveau and P. Greiner, On a geometric formula for the fundamental solution of subelliptic Laplacians, Math. Nachr. 181 (1996), pp. 81-163.

[25] R. Beals, B. Gaveau and P. Greiner, The Green function of model step two hypoelliptic operators and the analysis of certain tangential Cauchy Riemann complexes, Adv. Math. 121 (1996), pp. 288-345.

[26] R. Beals, B. Gaveau and P. Greiner, Complex Hamiltonian mechanics and parametrices for subelliptic Laplacians I, II and III, Bull. Sci. Math. 121 (1997), pp. 1-36, 97-149 and 195-259.

[27] R. Beals, B. Gaveau and P. Greiner, Hamilton-Jacobi theory and the heat kernel on Heisenberg groups, J. Math. Pures Appl. 79 (2000), pp. 633-689. 
[28] D. Békollé, C. Berger, C. A., L. and K. Zhu, BMO in the Bergman metric on bounded symmetric domains, J. Funct. Anal. 93 (1990), pp. 310-350.

[29] F. A. Berezin, Covariant and contravariant symbols of operators, (Russian) Izv. Akad. Nauk SSSR Ser. Mat. 36 (1972), pp. 1134-1167.

[30] F. A. Berezin, Spectral properties of generalized Toeplitz matrices, (Russian) Mat. Sb. (N.S.) 95 (137) (1974), pp. 305-325, 328.

[31] F. A. Berezin, General concept of quantization, Comm. Math. Phys. 40 (1975), pp. 153174.

[32] F. A. Berezin and M. A. Šubin, Symbols of operators and quantization, Hilbert space operators and operator algebras (Proc. Internat. Conf., Tihany, 1970), Colloq. Math. Soc. Janos Bolyai no. 5 (1972), pp. 21-52.

[33] C. Berger and L. Coburn, Toeplitz operators and quantum mechanics, J. Funct. Anal. 68 (1986), pp. 273-299.

[34] C. Berger and L. Coburn, Toeplitz operators on the Segal-Bargmann space, Trans. Amer. Math. Soc. 301 (1987), pp. 813-829.

[35] C. Berger and L. Coburn, Heat flow and Berezin-Toeplitz estimates, Amer. J. Math. 116 (1994), pp. 563-590.

[36] S. Bergman, Über die Entwicklung der harmonischen funktionen der Ebene und des Raumes nach orthogonalfunktionen, Math. Ann. 86 (1922), pp. 237-271.

[37] S. Bergman, Über die Kernfunktion eines Bereiches und ihr Verhalten am Rande, J. Reine Angew. Math. 169 (1933), pp. 1-42, and 172 (1934), pp. 389-128.

[38] S. Bergman, Sur quelques propriétés des transformations par un couple des fonctions de deux variables complexes, Atti della Accademia Nazionali dei Lincei, Rendiconti 19 ser. 6 (1934), pp. 474-478.

[39] S. Bergman, Zur Theorie von pseudokonformen Abbildungen, Rec. Math. (Mat. Sbornik) N.S. 1 (1936), pp. 79-96.

[40] S. Bergman, Partial differential equations, advanced topics, Brown University, Providence, 1941.

[41] S. Bergman, A remark on the mapping of multiply-connected domains, Amer. J. Math. 68 (1946), pp. 20-28.

[42] S. Bergman, Sur les functions orthogonales de plusieurs variables complexes avec les applications á la théorie des fonctions analytiques, Mémorial des Sciences Mathématiques $106,1947$. 
[43] S. Bergman, The kernel function and conformal mapping, Mathematical Survey no. 5, Amer. Math. Soc., 1950.

[44] S. Bergman and M. Schiffer, A representation of Green's and Neumann's functions in the theory of partial differential equations of second order, Duke Math. J. 14 (1947), pp. 609-638.

[45] S. Bergman and M. Schiffer, Kernel functions in the theory of partial differential equations of elliptic type, Duke Math. J. 15 (1948), pp. 535-566.

[46] S. Bergman and M. Schiffer, Kernel functions and elliptic differential equations in mathematical physics, Academic Press Inc., New York N. Y., 1953.

[47] A. Berlinet and C. Thomas-Agnan, Reproducing kernel Hilbert spaces in probability and statistics, Kluwer Academic, 2004.

[48] C. de Boor and R. E. Lynch, On splines and their minimum properties, J. Math. Mech. 15 (1966), pp. 953-970.

[49] D. Borthwick, A. Lesniewski and H. Upmeier, Non-perturbative deformation quantization of Cartan domains, J. Funct. Anal. 113 (1993), pp. 153-176.

[50] L. Boutet de Monvel, On the index of Toeplitz operators of several complex variables, Invent. Math. 50 no. 3 (1978/79), pp. 249-272.

[51] L. Boutet de Monvel, Toeplitz operators: an asymptotic quantization of symplectic cones. Stochastic processes and their applications in mathematics and physics (Bielefeld, 1985), Math. Appl. vol. 61 (1990), pp. 95-106.

[52] L. Boutet de Monvel and V. Guillemin, The spectral theory of Toeplitz operators, Annals of Mathematics Studies vol. 99, Princeton University Press, 1981.

[53] L. Boutet de Monvel and J. Sjöstrand, Sur la singularité des noyaux de Bergman et de Szegö, Journées: Équations aux Dérivées Partielles de Rennes, Asterisque no. 34-35 (1975), pp. 123-164.

[54] A. Brown and P. Halmos, Algebraic properties of Toeplitz operators, J. Reine Angew. Math. 213 (1963/1964), pp. 89-102.

[55] O. Calin, D. C. Chang and I. Markina, Generalized Hamilton-Jacobi equation and heat kernel on step two nilpotent Lie groups, Analysis and mathematical physics, 49-76, Trends Math., Birkhäuser, Basel, 2009.

[56] O. Calin, D. C. Chang, K. Furutani and C. Iwasaki, Heat kernels for elliptic and subelliptic operators, Methods and techniques, Applied and Numerical Harmonic Analysis 2011 
[57] E. Cartan, Sur les domaines bornés homogènes de l'espace de n-variables complexes, $A b h$. Math. Sem. Univ. Hamburg 11 (1935), pp. 116-162.

[58] D. C. Chang, S. C. Chang and J. Tie, Laguerre calculus and Paneitz operator on the Heisenberg group, Sci. China Ser. A 52 (2009), pp. 2549-2569,

[59] D. C. Chang and I. Markina, Anisotropic quaternion Carnot groups: geometric analysis and Green function, Adv. in Appl. Math. 39 (2007), pp. 345-394.

[60] B.R. Choe, H. Koo and Y.J. Lee, Commuting Toeplitz operators on the polydisk, Trans. Amer. Math. Soc. 356 (2004), pp. 1727-1749.

[61] B.R. Choe and Y.J. Lee, Pluriharmonic symbols of commuting Toeplitz operators, Illinois J. Math. 37 (1993), pp. 424-436.

[62] L. Coburn, Deformation estimates for the Berezin-Toeplitz quantization, Comm. Math. Phys. 149 (1992), pp. 415-424.

[63] L. Coburn, On the Berezin-Toeplitz calculus, Proc. Amer. Math. Soc. 129 (2001), pp. 3331-3338.

[64] L. Coburn, J. Isralowitz and Bo Li, Toeplitz operators with BMO symbols on the SegalBargmann space, Trans. Amer. Math. Soc. 363 (2011), pp. 3015-3030.

[65] R. R. Coifman and S. Lafon, Geometric harmonics: a novel tool for multiscale out-ofsample extension of empirical functions, Applied and Computational Harmonic Analysis vol. 21 no.1 (2006), pp. 31-52.

[66] Z. Čučković and N.V. Rao, Mellin transform, monomial symbols and commuting Toeplitz operators, J. Funct. Anal. 154 (1998), pp. 195-214.

[67] J. Cygan, Heat kernels for class 2 nilpotent groups, Studia Math. 64 (1979), pp. 227-238.

[68] E.B. Davies, Spectral theory and differential operators, Cambridge Studies in Advanced Mathematics, 42. Cambridge University Press, Cambridge, 1995.

[69] R.G. Douglas, Banach algebra techniques in operator theory, Graduate texts in mathematics, Springer, 1998.

[70] J. Duchon, Splines minimizing rotation invariant semi-norms in Sobolev spaces, Constructive theory of functions of several variables 1 (1977), pp. 85-100.

[71] A. J. Duran, The Stieltjes moments problem for rapidly decreasing functions, Proc. Amer. Math. Soc. 107 (1989), pp. 731-741.

[72] P. L. Duren and A. Schuster, Bergman spaces, Mathematical surveys and monographs, Amer. Math. Soc., 2004. 
[73] Ju. V. Egorov, Subelliptic operators, (Russian) Uspehi Mat. Nauk 30 (1975), pp. 57-114.

[74] M. Engliš, Some density theorems for Toeplitz operators on Bergman spaces, Czechoslovak Math. J. 40 (115) (1990), pp. 491-502.

[75] M. Engliš, Functions invariant under the Berezin transform, J. Funct. Anal. 121 (1994), pp. 233-254.

[76] M. Engliš, Berezin quantization and reproducing kernels on complex domains, Trans. Amer. Math. Soc. 348 (1996), pp. 411-479.

[77] M. Engliš, Compact Toeplitz operators via the Berezin transform on bounded symmetric domains, Integr. Equ. Oper. Theory 33 (1999), pp. 426-455.

[78] N. S. Faour, Toeplitz operators on Bergman spaces, Rend. Circ. Mat. Palermo (2) 35 (1986), pp. 221-232.

[79] J. Faraut, S. Kaneyuki, A. Korányi, Qi-keng Lu and G. Roos, Analysis and geometry on complex homogeneous domains, Progress in Mathematics, 185, Birkhäuser Boston, Inc., Boston, MA, 2000.

[80] J. Faraut and A. Korányi, Function spaces and reproducing kernels on bounded symmetric domains, J. Funct. Anal. 88 (1990), pp. 64-89.

[81] C. Fefferman, The Bergman kernel and biholomorphic mappings of pseudoconvex domains, Invent. Math. 26 (1974), pp. 1-65.

[82] E. Fischer, Über die differentiatensprozesse der algebra, J. Math. 148 (1917), pp. 1-78.

[83] V. Fock, Verallgemeinerung and lösung der Diracshen statistischen gleichung, Z. Phys. 49 (1928), pp. 339-357.

[84] G.B. Folland, Harmonic analysis in phase space, Annals of mathematics studies, Princeton University Press, 1989.

[85] K. Furutani, The heat kernel and the spectrum of a class of nilmanifolds. Comm. Partial Differential Equations 21 (1996), no. 3-4, pp. 423-438.

[86] K. Furutani, Heat kernels of the sub-Laplacian and the Laplacian on nilpotent Lie groups. Analysis, geometry and topology of elliptic operators, World Sci. Publ., Hackensack, NJ (2006), pp. 173-214.

[87] B. Gaveau, Solutions fondamentales, représentations, et estimes sous-elliptiques pour les groupes nilpotents d'ordre 2, (French) C. R. Acad. Sci. Paris Sér. A-B 282 (1976), pp. 563-566. 
[88] B. Gaveau, Principe de moindre action, propagation de la chaleur et estimées sous elliptiques sur certains groupes nilpotents, (French) Acta Math. 139 (1977), pp. 95-153.

[89] F. Geng and M. Cui, Solving singular nonlinear boundary value problems by combining the homotopy perturbation method and reproducing kernel Hilbert space method, Int. J. Comput. Math. 87 (2010), pp. 2024-2031.

[90] F. Geng, A new reproducing kernel Hilbert space method for solving nonlinear fourthorder boundary value problems, Appl. Math. Comput. 213 (2009), pp. 163-169.

[91] F. Geng, Solving singular second order three-point boundary value problems using reproducing kernel Hilbert space method, Appl. Math. Comput. 215 (2009), pp. 2095-2102.

[92] S. Grudsky, A. Karapetyants and N. Vasilevski, Toeplitz Operators on the unit Ball in $\mathbb{C}^{n}$ with radial symbols, Journal of Operator Theory 49 (2003), pp. 325-346.

[93] S. Grudsky, R. Quiroga-Barranco and N. Vasilevski, Commutative $C^{*}$-algebras of Toeplitz operators and quantization on the unit disc, J. Funct. Anal. 234 (2006), pp. 1-44.

[94] S. Grudsky and N. Vasilevski, Bergman-Toeplitz operators: radial component influence, Integr. Equ. Oper. Th. 40 (2001), pp. 16-33.

[95] S. Grudsky and N. Vasilevski, Toeplitz operators on the Fock space: radial component effects, Integr. Equ. Oper. Th. 44 (2002), pp. 10-37.

[96] S. Grudsky and N. Vasilevski, On the structure of the $C^{*}$-algebra generated by Toeplitz operators with piece-wise continuous symbols, Complex Analysis and Operator Theory 2 (2008), pp. 525-548.

[97] S. Grudsky and N. Vasilevski, Anatomy of the $C^{*}$-algebra generated by Toeplitz operators with piece-wise continuous symbols, Operator Theory: Advances and Applications 190 (2009), pp. 243-265.

[98] V. Guillemin, Toeplitz operators in n-dimensions, Integr. Equ. Oper. Th. 7 (1984), pp. 145-205.

[99] B. C. Hall, Holomorphic methods in analysis and mathematical physics, In: First Summer School in Analysis and Mathematical physics, Eds. Pérez-Esteva and C. Villegas-Blas, Contemp. Math. vol. 260, Amer. Math. Soc., Providence, R. I. (2000), pp.1-59.

[100] M. Ha Quang, S. Kang and T. M. Le, Image and video colorization using vector-valued reproducing kernel Hilbert spaces, J. Math. Imaging Vision 37 (2010), pp. 49-65.

[101] H. Hedenmalm, B. Korenblum and K. Zhu, Theory of Bergman spaces, Graduate texts in mathematics, Springer, 2000. 
[102] S. Helgason, Differential Geometry, Lie Groups, and Symmetric Spaces, American Mathematical Society, 2001.

[103] K. Hirachi, Invariant theory for Bergman kernels on strongly pseudoconvex domains, (Japanese) Sūgaku 52 (2000), pp. 360-375.

[104] K. Hirachi, A link between the asymptotic expansions of the Bergman kernel and the Szeg kernel. Complex analysis in several variables-Memorial Conference of Kiyoshi Oka's Centennial Birthday, Adv. Stud. Pure Math., Math. Soc. Japan, Tokyo 42 (2004), pp. 115-121.

[105] Y. C. Hon and T. Takeuchi, Discretized Tikhonov regularization by reproducing kernel Hilbert space for backward heat conduction problem, Adv. Comput. Math. 34 (2011), pp. 167-183.

[106] L. Hörmander, The analysis of linear partial differential operators IV, Berlin, Heidelberg, New York: Springer, 1985.

[107] L. Hörmander, Hypoelliptic second order differential equations, Acta Math. 119 (1967), pp. 147-171.

[108] L. Hörmander, Differential operators of principal type, Math. Ann. 140 (1960), pp. 124146.

[109] R. Horn and C. Johnson, Matrix analysis, Cambridge University Press, Cambridge, 1985.

[110] A. Hulanicki, The distribution of energy in the Brownian motion in the Gaussian field and analytic-hypoellipticity of certain subelliptic operators on the Heisenberg group, Studia Math. 56 (1976), 165-173.

[111] J. Isralowitz, Compact Toeplitz operators on the Segal-Bargmann space, J. Math. Anal. Appl. 374 (2011), pp. 554-557.

[112] J. Isralowitz and K. Zhu, Toeplitz operators on the Fock space, Integr. Equ. Oper. Th. 66 (2010), pp. 593-611.

[113] H. Issa, Compact Toeplitz operators for weighted Bergman spaces on bounded symmetric domains. Integr. Equ. Oper. Th. 70 (2011), pp. 569-582.

[114] C. Iwasaki, A proof of the Gauss-Bonnet-Chern theorem by the symbolic calculus of pseudo-differential operators, Japan. J. Math. (N.S.) 21 (1995), pp. 235-285.

[115] C. Iwasaki, Symbolic calculus for construction of the fundamental solution for a degenerate equation and a local version of Riemann-Roch theorem, Geometry, analysis and applications (Varanasi, 2000), World Sci. Publ., River Edge, NJ (2001), pp. 83-92. 
[116] C. Iwasaki and N. Iwasaki, Parametrix for a degenerate parabolic equation and its application to the asymptotic behavior of spectral functions for stationary problems. Publ. Res. Inst. Math. Sci. 17 (1981), pp. 577-655.

[117] J. Janas, Unbounded Toeplitz operators in the Segal-Bargmann space, Studia Math. 99 no. 2 (1991), pp. 87-99.

[118] J. Janas and J. Stochel, Unbounded Toeplitz operators in the Segal-Bargmann space. II, J. Funct. Anal. 126 (1994), pp. 418-447.

[119] M. de Jeu, Determinate multidimensional measures, the extended Carleman theorem and quasi-analytic weights, Ann. Probab. 31 (2003), pp. 1205-1227.

[120] A. Klinger, New derivation of the Heisenberg kernel, Communications in Partial Differential Equations 22 (1997), pp. 2051-2060.

[121] A. N. Kolmogorov, Stationary sequences in Hilbert space, Bull. Math. Univ. Moscow 2 (1941), pp. 1-40.

[122] B. Korenblum and K. Zhu, An application of Tauberian theorems to Toeplitz operators, J. Operator Theory 33 (1995), pp. 353-361.

[123] M. Koecher, An elementary approach to bounded symmetric domains, Rice University, Houston, 1969

[124] S. G. Krantz, Function theory of several complex variables, AMS Chelsea Publishing Series, Amer. Math. Soc., 2001.

[125] O. Kures and K. Zhu, A class of integral operators on the unit ball of $\mathbb{C}^{n}$, Integr. Equ. Oper. Th. 56 (2006), pp. 71-82.

[126] T. Le, The commutants of certain Toeplitz operators on weighted Bergman spaces, $J$. Math. Anal. Appl. 348 (1) (2008), pp. 1-11.

[127] M. Loéve, Probability theory, Van Nostrand, Princeton N. J. 2nd edition, 1960.

[128] O. Loos, Bounded symmetric domains and Jordan pairs. Mathematical Lectures, University of California at Irvine, 1977.

[129] I. Louhichi and N.V. Rao, Bicommutants of Toeplitz operators, Arch. Math. 91 (2008), pp. 256-264.

[130] I. Louhichi and L. Zakariasy, On Toeplitz operators with quasihomogeneous symbols, Arch. Math. 85 (2005), pp. 248-257.

[131] X. Ma and G. Marinescu, Holomorphic Morse inequalities and Bergman kernels, Progress in Mathematics, 254. Birkhäuser Verlag, Basel, 2007. 
[132] A.I. Markushevich, Theory of Functions of a Complex Variable, AMS Chelsea Publishing Vol. 1, 1977.

[133] J. Mercer, Functions of positive and negative type and their connection with the theory of integral equations, Phil. Trans. R. Soc. Lond. A. 209 (1909), pp. 415-446.

[134] J. Mercer, Sturm-Louville series of normal functions in the theory of integral equations, Philos. Trans. Roy. Soc. London ser. A vol. 211 (1911), pp. 111-198.

[135] E. H. Moore, On properly positive Hermitian matrices, Bull. Amer. Math. Soc. 23 (1916), pp. 59.

[136] E. H. Moore, General analysis, Memoirs of the American Philosophical Society, Part I 1935, Part II 1939.

[137] A. Nagel and W. Rudin, Moebius-invariant function spaces on balls and spheres, Duke Math. J. 43 (1976), pp. 841-865.

[138] R. Narasimhan, Several Complex Variables, Chicago Lectures in Mathematics vol. 138, 1971.

[139] Y. A. Neretin, Lectures on Gaussian integral operators and classical groups, EMS Series of Lectures in Mathematics. European Mathematical Society (EMS), Zürich, 2011.

[140] G. M. Nielson, Multivariate smoothing and interpolating splines, SIAM Journal on Numerical Analysis vol. 11 no. 2 ( 1974), pp. 435-446.

[141] E. Parzen, Time series analysis papers, Holden-Day, San Francisco, 1967.

[142] E. Parzen, Statical inference on time series by RKHS methods, Proc. of 12th Biennial Seminar of the Canadian Mathematical Congress, Amer. Math. Soc. Providence (1970), pp. 1-37.

[143] J. C. T. Pool, Mathematical aspects of the Weyl correspondence, J. Mathematical Phys. 7 (1966), pp. 66-76.

[144] R. Quiroga-Barranco and N. Vasilevski, Commutative $C^{*}$-algebras of Toeplitz operators on the unit ball, I. Bargmann-type transforms and spectral representations of Toeplitz operators, Integr. Equ. Oper. Th. 59 (3) (2007), pp. 379-419.

[145] R. Quiroga-Barranco and N. Vasilevski, Commutative $C^{*}$-algebras of Toeplitz operators on the unit ball, II. Geometry of the level sets of symbols,Integr. Equ. Oper. Th. 60 (2008), pp. 89-132.

[146] D. B. Ray and I. M. Singer, $R$-torsion and the Laplacian on Riemannian manifolds, Advances in Math 7 (1971), pp. 145-210. 
[147] L. Rodino, Linear partial differential operators in Gevrey spaces, World Scientific Publishing Co. Inc., River Edge, NJ, 1993.

[148] L. P. Rothschild and E. M. Stein, Hypoelliptic differential operators and nilpotent groups, Acta Math. 137 (1976), pp. 247-320.

[149] W. Rudin, Function theory in the unit ball of $\mathbb{C}^{n}$, A series of comprehensive studies in Mathematics, Springer, 1991.

[150] L. Schwartz, Sous-espaces hilbertiens d'espaces vectoriels topologiques et noyaux associés (noyaux reproduisants), J. Analyse Math. 13 (1964), pp. 115-256.

[151] S. Saitoh, Theory of reproducing kernels and its applications, Pitman research notes in mathematics series, Longman Scientific \& Technical, 1988.

[152] I. E. Segal, Mathematical problems of relativistic physics, Amer. Math. Soc., Providence, 1963.

[153] I. E. Segal, The complex wave representation of the free Boson filed. Topics in Functional Analysis: (essays dedicated to M. G. Krein on the occasion of his 70th birthday), Adv. in Math. Suppl. Stud., 3, Academic Press, New York-London (1978), pp. 321-344.

[154] I.H. Sneddon, The use of integral transforms, University of Glasgow, Glasgow, 1972.

[155] S. B. Sontz, Entropy and the Segal-Bargmann transform, Journal of Mathematical Physics vol. 39 (1998), pp. 2402-2417.

[156] E. M. Stein and G. L. Weiss, Introduction to Fourier analysis on Euclidean spaces, Princeton mathematical series, Princeton University Press, 1971.

[157] E. M. Stein and R. Shakarchi, Real analysis: measure theory, integration and Hilbert spaces, Princeton lectures in analysis III, Princeton University Press, 2005.

[158] D. Suárez, The essential norm of operators in the Toeplitz algebra on $A^{p}\left(\mathbb{B}_{n}\right)$, Indiana Univ. Math. J. 56 (2007), pp. 2185-2232.

[159] T. Takeuchi and M. Yamamoto, Tikhonov regularization by a reproducing kernel Hilbert space for the Cauchy problem for an elliptic equation, SIAM J. Sci. Comput. 31 (2008), pp. 112-142.

[160] L. Takhtajan, Quantum mechanics for mathematicians, Graduate studies in mathematics, American Mathematical Society, 2008.

[161] J. Tie, The twisted Laplacian on $\mathbb{C}^{n}$ and the sub-Laplacian on $H_{n}$, Comm. Partial Differential Equations 31 (2006), pp. 1047-1069. 
[162] J. Tie and M. W. Wong, Wave kernels of the twisted Laplacian, Modern trends in pseudodifferential operators, Oper. Theory Adv. Appl., Birkhäser, Basel 172, pp. 107-115, 2007.

[163] O. Toeplitz, Zur Theorie der quadratischen und bilinearen Formen von unendlichvielen Veränderlichen, Math. Ann. 70 (1911), pp. 351-376.

[164] S. Thangavelu, Lectures on Hermite and Laguerre expansions, Mathematical Notes, 42. Princeton University Press, Princeton, NJ, 1993.

[165] S. Thangavelu, Harmonic analysis on the Heisenberg group, Progress in Mathematics, 159. Birkhäuser Boston, Inc., Boston, MA, 1998.

[166] H. Upmeier, Toeplitz operators and index theory in several complex variables, Operator Theory: Advances and Applications, Birkhäuser Verlag, 1996.

[167] N. Vasilevski, Poly-Fock spaces, Operator Theory: Advances and Applications 117 (2000), pp. 371-386.

[168] N. Vasilevski, Bergman space structure, commutative algebras of Toeplitz operators and hyperbolic geometry, Integr. Equ. Oper. Th. 46 (2003), pp. 235-251.

[169] N. Vasilevski, Commutative algebras of Toeplitz Operators on the Bergman space, Operator Theory: Advances and Applications, Birkhäser, 2003.

[170] N. Vasilevski, Commutative algebras of Toeplitz operators and Berezin quantization. Non-commutative geometry in mathematics and physics, Contemp. Math., Amer. Math. Soc., Providence, RI 462 (2008), pp. 125-143.

[171] N. Vasilevski, Parabolic quasi-radial quasi-homogeneous symbols and commutative algebras of Toeplitz operators, Operator Theory: Advances and Applications 202 (2010), pp. 553-568.

[172] N. Vasilevski, Quasi-radial quasi-homogeneous symbols and commutative Banach algebras of Toeplitz operators, Integr. Equ. Oper. Th. 66 (2010), pp. 141-152.

[173] G. Wahba, Spline models for observational data, SIAM, Philadelphia, 1990.

[174] N. R. Wallach, Symplectic geometry and Fourier analysis in Lie Groups: History, Frontiers and Applications, Vol. V. Math Sci Press, Brookline, Mass., 1977.

[175] E.T. Whittaker and G.N. Watson, A course of modern analysis, Cambridge University Press, London, 1958.

[176] D. V. Widder, The Laplace transform, Princeton mathematical series 6, Princeton university press, 1946. 
[177] M. W. Wong, The heat equation for the Hermite operator on the Heisenberg group, Hokkaido Math. J. 34 (2005), pp. 393-404.

[178] Y. Yang and T. Qian, An elementary proof of the Paley-Wiener theorem in $\mathbb{C}^{m}$, Complex Variables and Elliptic Equations 51 (2006), 599-609.

[179] R. M. Young, An introduction to nonharmonic Fourier series, Pure and applied mathematics, Academic Press, 1980.

[180] S. Zaremba, L'équation biharmonique et une classe remarquable de fonctions fondamentales harmoniques, Bulletin International de l’Académie des Sciences de Cracovie (1907), pp. 147-196.

[181] Ze-Hua Zhou and Xing-Tang Dong, Algebraic properties of Toeplitz operators with radial symbols on the Bergman space of the unit ball, Integr. Equ. Oper. Th. 64 (2009), pp. 137-154.

[182] F. Zhu, The heat kernel and the Riesz transforms on the quaternionic Heisenberg groups, Pacific J. Math. 209 (2003), pp. 175-199.

[183] K. Zhu, Positive Toeplitz operators on weighted Bergman spaces of bounded symmetric domains, J. Operator Theory 20 (1988), pp. 329-357.

[184] K. Zhu, BMO and Hankel operators on Bergman spaces, Pacific J. Math. 155 (1992), pp. 377-397.

[185] K. Zhu, Spaces of holomorphic functions in the unit ball, Graduate texts in mathematics, Springer, 2005.

[186] K. Zhu, Operator theory in function spaces, Mathematical surveys and monographs, Amer. Math. Soc., 2007.

[187] N. Zorboska, Toeplitz operators with BMO symbols and the Berezin transform, Int. J. Math. Math. Sci. (2003), pp. 2929-2945. 


\section{Curriculum Vitae}

$\begin{array}{ll}\text { Name: } & \text { Hassan Issa } \\ \text { Address: } & \text { Untere Maschstraße 13, } 37073 \text { Göttingen } \\ \text { Date of birth: } & \text { 15.08.1986 } \\ \text { Nationality: } & \text { Lebanese } \\ \text { Marital status: } & \text { Married }\end{array}$

\section{SCHOOL AND EDUCATION}

1996 - 2000 Primary School: Lycée Patriot, Beirut, Lebanon.

2000 - 2003 Secondary school: Al Bir wl Ihsan, Beirut, Lebanon.

2003 General qualification for university entrance speciality

General Science, Beirut, Lebanon.

\section{Educational history}

2004 - 2007 Maitrise (3 years Bachelor +1) of Sciences in pure mathematics at the Université Libanaise, Faculté des Sciences, Lebanon.

2007 - 2008 Master in Sciences in applied and fundamental mathematics at the Université d'Angers and Université de Nantes, France. Master thesis ,, On the classification of Jacobi PDE systems”.

\section{Employment history}

Nov 2008- Feb 2009 Internship in École publique d'ingénieurs et centre de recherche at the Université de Brest, France.

SS 2009 - WS 2009/2010 teaching at the Ernst-Moritz-Arndt Universität in Greifswald, Germany

$2009-2010$ research assistant at the Ernst-Moritz-Arndt Universität in Greifswald, Germany.

2010 - Present research assistant at the Georg-August-Universität in Göttingen, Germany. 